\title{
Pathogenesis of orthopoxvirus (OPXV) infection in common CM and identification of immune correlates after vaccination with differently attenuated vaccines
}

\author{
Dis sertation \\ for the award of the degree \\ "Doctor rerum naturalium" \\ Division of Mathematics and Natural Sciences \\ of the Georg-August-Universität Göttingen
}

within the doctoral program

Emerging Infectious Diseases (EIDIS) in the Leibniz Graduate School of the Georg-August University School of Science (GAUSS)

submitted by

\section{Li Lin Gan}

from Geesthacht, Germany

Göttingen, 2017 



\section{Thesis Committee}

Dr. Christiane Stahl-Hennig, Unit of Infection Models, German Primate Center, Göttingen

Prof. Dr. Dr. Claus-Peter Czerny, Division of Microbiology and Animal Hygiene, Department of Animal Sciences, Institute of Veterinary Medicine, Georg-August University Göttingen, Göttingen

Prof. Dr. Stephan Becker, Institute for Virology, Philipps-University Marburg, Marburg

\section{Members of the Examination Board}

Referee: Dr. Christiane Stahl-Hennig, Unit of Infection Models, German Primate Center, Göttingen

$2^{\text {nd }}$ Referee: Prof. Dr. Dr. Claus-Peter Czerny, Division of Microbiology and Animal Hygiene, Department of Animal Sciences, Institute of Veterinary Medicine, Georg-August University Göttingen

\section{Further members of the Examination Board}

Prof. Dr. Stefan Pöhlmann, Infection Biology Unit, German Primate Center, Göttingen

Prof. Dr. Lutz Walter, Primate Genetics Laboratory, German Primate Center, Göttingen

Dr. Alexander Hahn, Junior Research Group Herpesviruses, German Primate Center, Göttingen

Dr. Christian Roos, Primate Genetics Laboratory, German Primate Center, Göttingen 



\section{Contents}

Table of contents......................................................................................................................

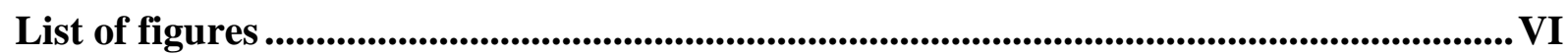

List of tables............................................................................................................................ IX

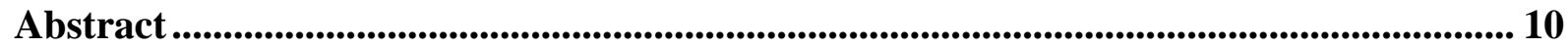

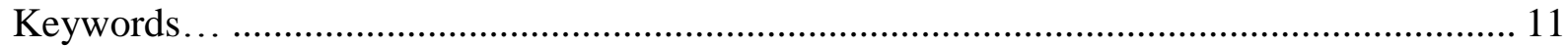

1 INTRODUCTION..................................................................................................... 12

1.1 Historical overview of orthopoxvirus infections.................................................. 12

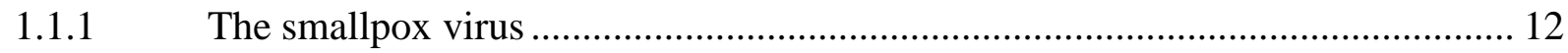

1.1.2 The potential threat of bioterrorism and zoonotic events ..................................... 15

$1.2 \quad$ Orthopoxvirus diseases ....................................................................................... 16

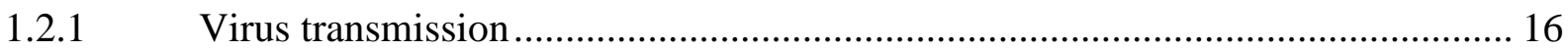

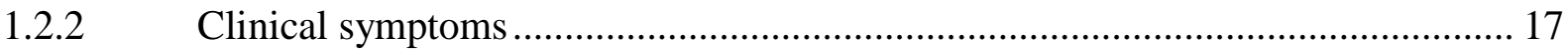

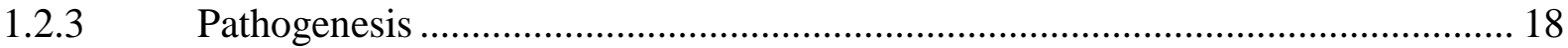

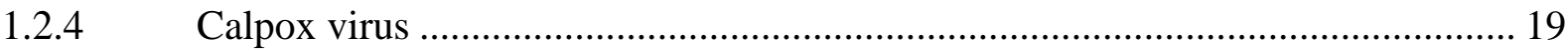

1.3 Orthopoxviruses within the Poxviridae family .................................................... 20

$1.4 \quad$ Virus structure and genome ............................................................................. 20

$1.5 \quad$ Virus life cycle....................................................................................................... 22

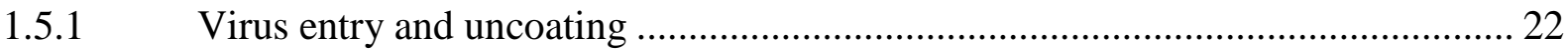

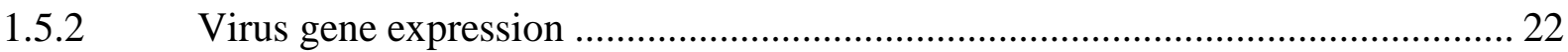

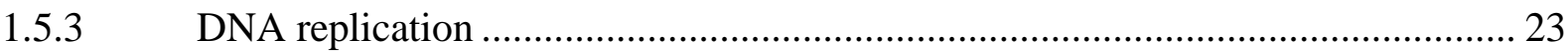

1.5.4 Virion assembly, maturation and release …………………………………... 23

$1.6 \quad$ Orthopoxviruses in humans ..................................................................... 24

1.6.1 Zoonotic poxvirus infections in humans ........................................................ 24

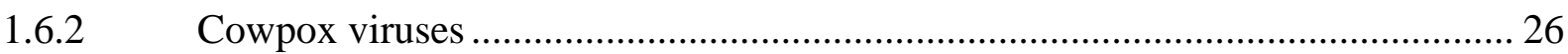

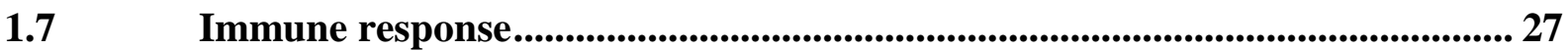

$1.8 \quad$ Immune correlates of smallpox virus protection............................................... 30

1.9 Prevention (vaccine) and treatment of OPXV infection ...................................31

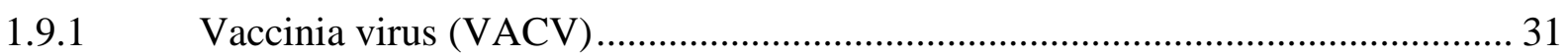

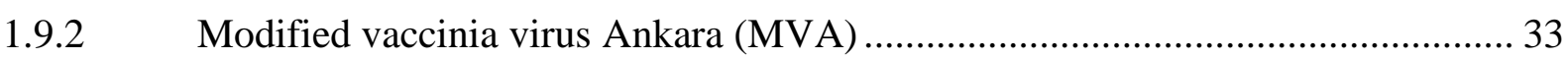

1.9.3 Modified vaccinia virus Tiantan (MVTT) _......................................................... 34

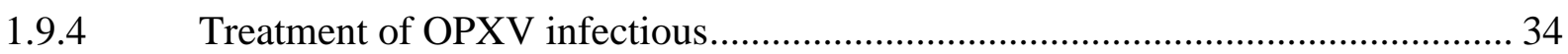

1.10 The common marmoset - Callithix jacchus ........................................................... 35

1.11 Animal models - a non-human primate model of OPXV infection....................36 
$1.12 \quad$ Aims and Scope................................................................................................... 37

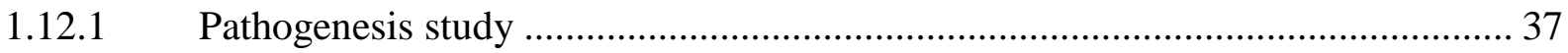

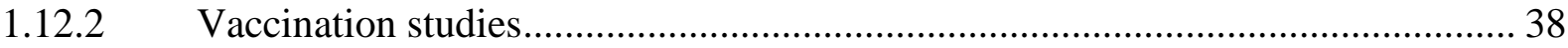

2 MATERIAL AND METHODS ................................................................................. 40

2.1 Material ........................................................................................................... 40

$2.2 \quad$ Animal experiments ...................................................................................... 44

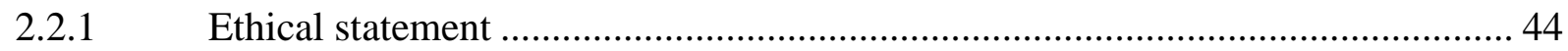

2.2.2 Non-human primates and animal husbandry ………………………………...... 44

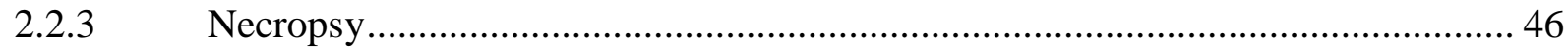

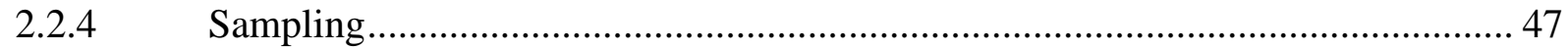

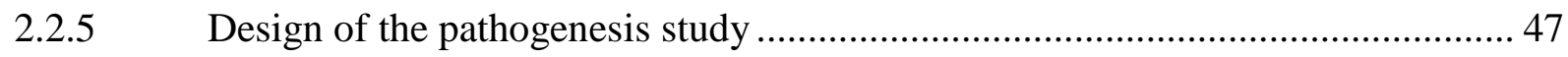

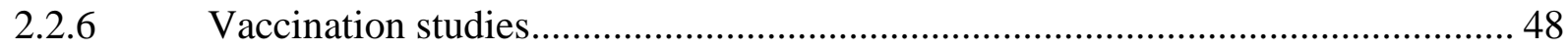

$2.3 \quad$ Cell culture ...................................................................................................................5 50

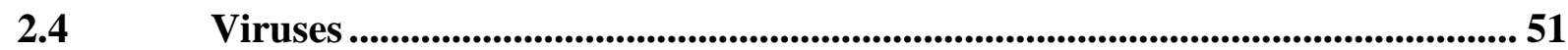

2.5 Isolation of peripheral blood mononuclear cells (PBMCs) and buffy coat from whole blood ...................................................................................................... 51

$2.6 \quad$ Extraction of nucleic acid ...........................................................................52

2.6.1 DNA extraction from blood samples.......................................................... 52

2.6.2 DNA extraction from tissue samples.............................................................. 52

$2.7 \quad$ Polychromatic flow cytomety ................................................................5 52

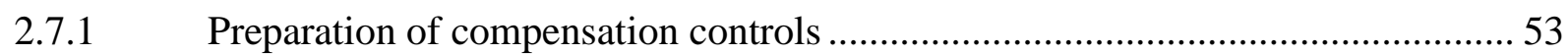

2.7.2 Polychromatic flow cytometric staining for calpox antigen in PBMCs.................53

2.7.3 Polychromatic flow cytometric calpox antigen staining of cells from buffy coat 54

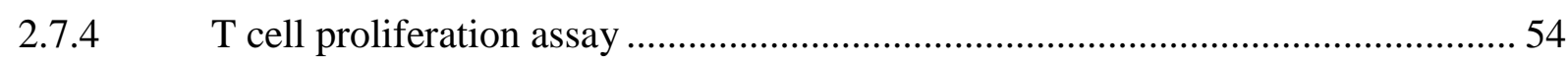

2.7.5 Polychromatic flow cytometric analysis of whole blood ..................................... 55

2.7.6 Polychromatic flow cytometric staining of whole blood for the determination of absolute numbers of different immune cell subsets .................................................. 56

$2.8 \quad$ Virus quantification .........................................................................................56

2.8.1 Virus quantification by endpoint dilution assay in tissue homogenates ............... 56

2.8.2 Virus quantification by endpoint dilution assay in buffy coat ............................. 57

$2.9 \quad$ Detection of calpox virus................................................................................. 58

2.10 Quantitative real-time PCR........................................................................... 59

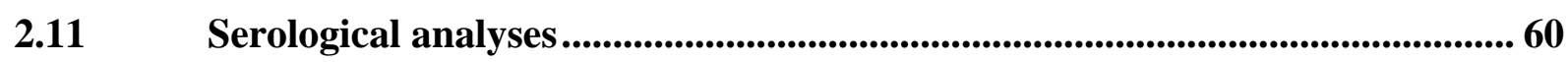

2.11.1 Binding antibodies by enzyme-linked immunosorbent assay (ELISA) ................ 60

2.11.2 B cell epitope fine mapping by peptide microarray ............................................ 62 


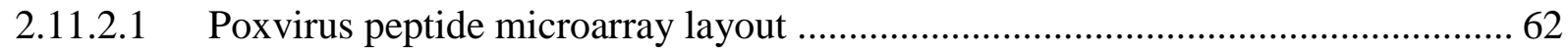

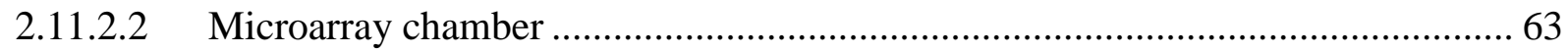

2.11.2.3 Validation of the chip using mAbs against known epitope regions ..................... 64

2.11.2.4 Poxvirus scanning chip in the calpox virus/marmoset model ............................... 65

2.11.2.5 Screening procedure and data acquisition of the microarray chip .......................65

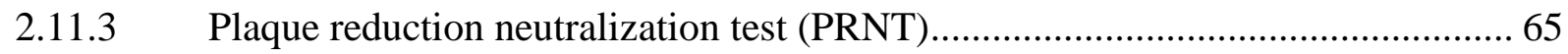

2.11.4 Gating strategy for the identification of innate and adaptive immune cells ......... 66

2.11.5 Gating strategy to assess absolute cell counts of innate and adaptive immune cells......

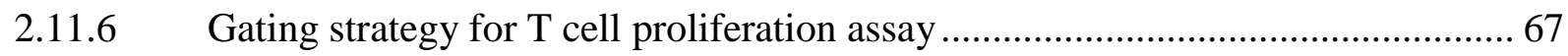

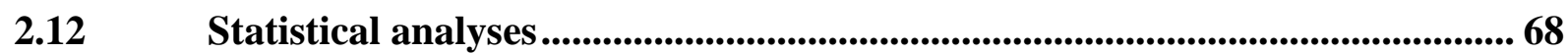

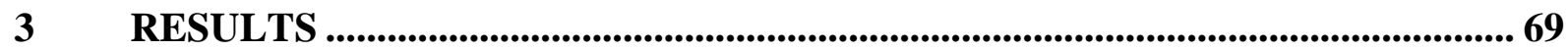

3.1 Pathogenesis study - portal of viral entry (I) ..................................................69

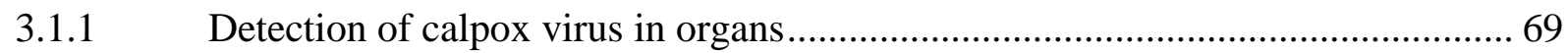

3.1.1.1 Replicating calpox virus and calpox DNA copies in different organs at different time points after infection.

3.2 Pathogenesis study - early dissemination and pathological sequelae of infection (II) .......................................................................................................... 71

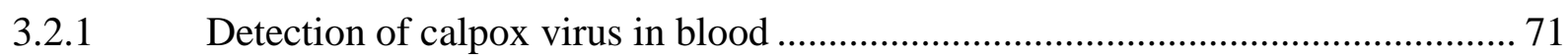

3.2.2 Detection of calpox virus antigen in various immune cells by flow cytometry .... 73

3.3 Vaccination studies - identification of immune correlates (II) .......................... 75

3.3.1 Immunization with MVTT showed highest vaccine efficacy ............................ 75

3.3.2 Post-challenge viral load confirms survival rates.............................................. 76

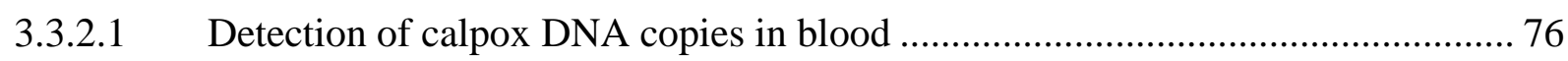

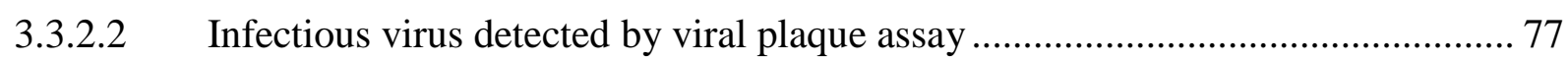

3.3.3 Humoral responses following immunization ................................................... 78

3.3.3.1 No significant influence of binding serum Abs against whole virus particles on

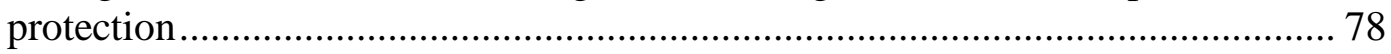

3.3.3.2 Successful validation of the poxvirus peptide microarray chip .......................... 81

3.3.3.3 High resolution mapping of antibody linear targets revealed unique epitopes in

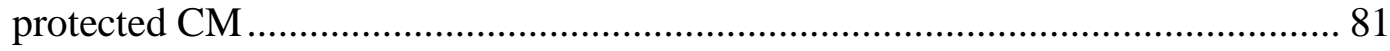

3.3.3.4 Neutralization antibodies did not correlate with protection ................................ 83

3.3.4 Phenotyping of immune cells and cellular immune response .............................. 86

3.3.4.1 Longitudinal phenotyping of innate and adaptive immune cells in whole blood . 86

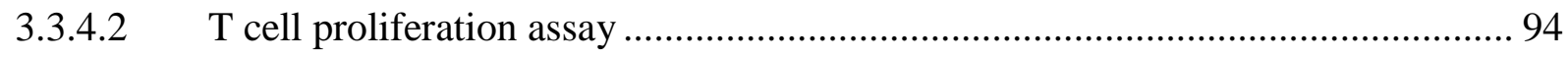

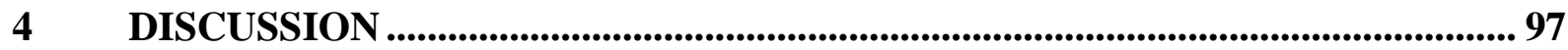


4.1 Pathogenesis study ...................................................................................................97

4.1.1 Following intranasal application calpox virus enters through the nasal

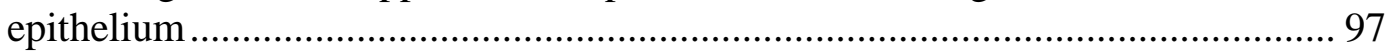

4.1.2 Identification of calpox virus replicating immune cells .................................... 98

4.1.3 Comparison of methods for the detection of calpox virus in blood samples ........ 99

4.1.4 (II) The early dissemination and pathological sequelae of infection .................. 100

4.1.5 Virus titration and viral DNA quantification of organ homogenates .................. 103

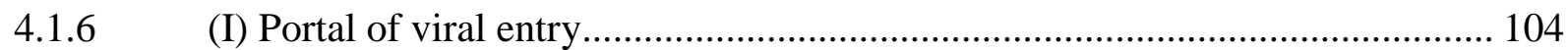

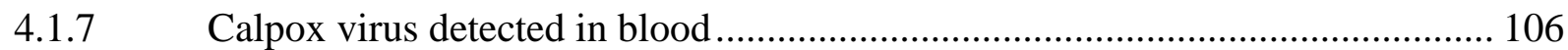

4.1.8 Detection of calpox virus infection with anti-vaccinia virus (VACV) serum..... 107

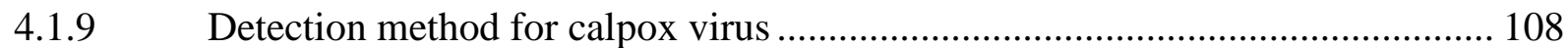

4.1.10 Onset of viremia is dose dependent as determined by real-time PCR, plaque assay

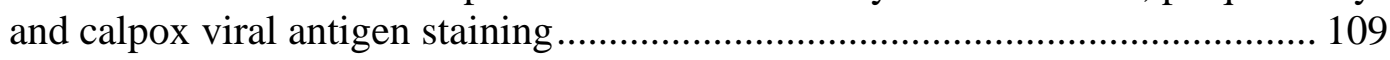

4.1.11 (II) Early dissemination and pathological sequelae of infection ......................... 109

$4.2 \quad$ Vaccination studies............................................................................................... 113

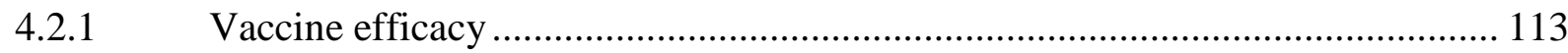

4.2.2 The role of the humoral immune response in the calpox virus/marmoset model 115

4.2.3 The role of the cellular immune response in the calpox virus/marmoset model. 119

(III) Identification of immune correlates .................................................... 120

4.3.1 Advantages and disatvantages of the calpox virus/marmoset model ................. 122

4.3.2 Relevance of modified vaccinia virus Ankara (MVA) and modified vaccinia virus Tiantan (MVTT) in developing a safer, but efficacious smallpox vaccine......... 123

4.3.3 The role of the different vaccination routes ................................................... 124

4.4 Conclusion and outlook......................................................................................... 125

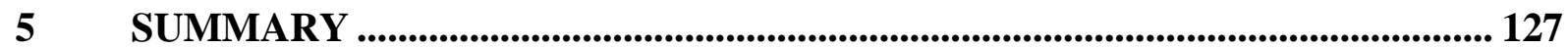

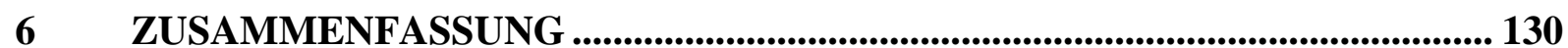

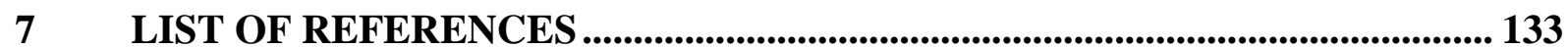

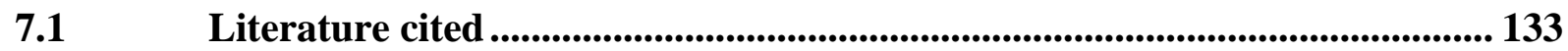

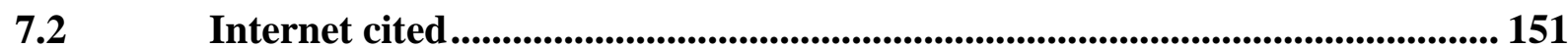

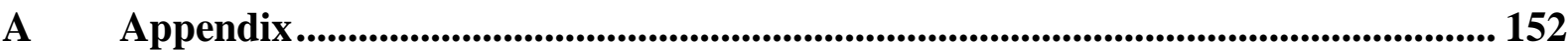

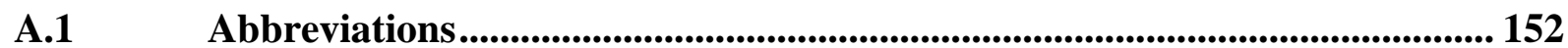

A.2 Tables and Protocols ........................................................................................... 155

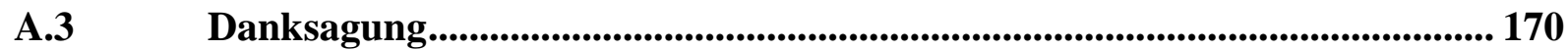

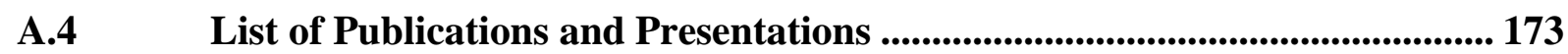

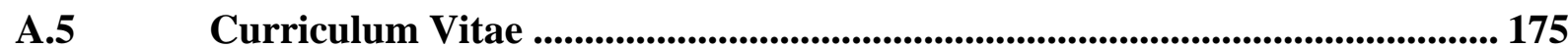




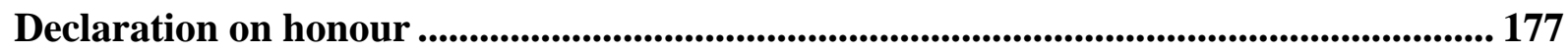




\section{List of figures}

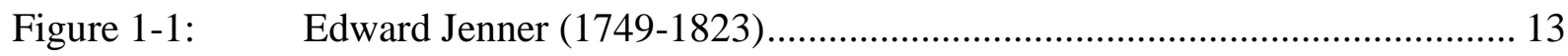

Figure 1-2: $\quad$ Location of the world's last known endemic case of smallpox ................... 15

Figure 1-3: $\quad$ First proven case of rat-to-human transmission of cowpox in 2002 ........... 17

Figure 1-4: $\quad$ Drawing of extracellular enveloped vaccinia virus particle ....................... 21

Figure 1-5: $\quad$ 7-year-old female child with the typical clinical presentation of human monkeypox, Sankuru District, Democratic Republic of Congo ................. 25

Figure 1-6: Cowpox virus-infected cotton-top tamarin ............................................ 27

Figure 1-7: $\quad$ Two adult Callithix jacchus individuals with twins ................................... 35

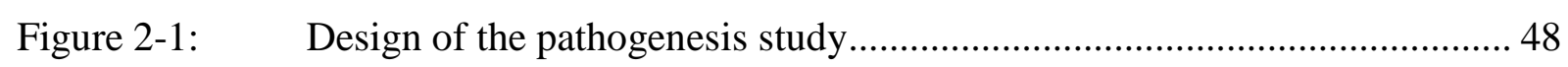

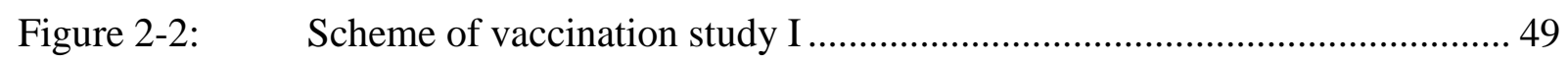

Figure 2-3: Scheme of vaccination study II............................................................. 50

Figure 2-4: $\quad$ Gating strategy for the detection of calpox virus antigen in immune cells in buffy coat by flow cytometry ............................................................ 59

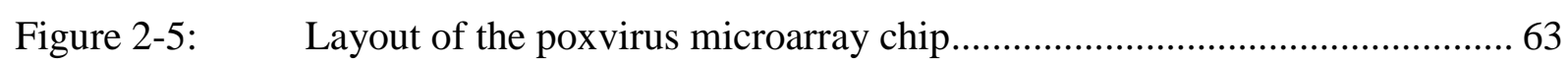

Figure 2-6: The poxvirus microarray chamber was designed to analyze four microarray chips simultaneously ....................................................................... 63

Figure 2-7: Rubber cover of the poxvirus microarray chamber's lid depicted from below

Figure 2-8: Gating strategy for the identification of innate and adaptive immune cells by flow cytometry......

Figure 2-9: Gating strategy for the identification of absolute counts of innate and adaptive immune cells by flow cytometry

Figure 2-10: $\quad$ Representative gating strategy of CFSE-stained $\mathrm{T}$ cells from ConA or MVA-stimulated PBMCs from vaccination study II.

Figure 3-1: $\quad$ Calpox virus DNA copy numbers and TCID 50 of infectious calpox virus in different tissues after i.n. infection

Figure 3-2: $\quad$ Calpox virus DNA copy numbers and TCID 50 of infectious calpox virus in different tissues three days after i.n. infection .....

Figure 3-3: $\quad$ Calpox virus DNA in blood was determined by real-time PCR at the indicated time points after infection

Figure 3-4: $\quad$ Replicating calpox virus in blood at different time points after infection by cocultivation of buffy coat with Vero E6 cells ......................................... 73

Figure 3-5: Calpox virus antigen was detected in immune cells of buffy coat by flow

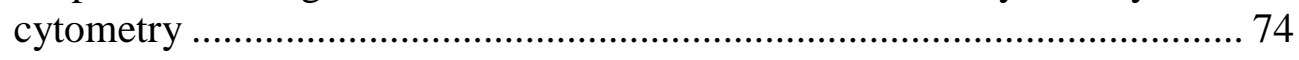

Figure 3-6: $\quad$ Exemplary dot plot showing calpox antigen positive $\left(\right.$ calpox $\left.^{+}\right)$immune cell populations in buffy coat ...

Figure 3-7: $\quad$ Kaplan-Meier plot on survival of vaccinated and unvaccinated CM after a lethal challenge with calpox virus........................................................ 75 
Figure 3-8: $\quad$ Calpox viral DNA in blood of vaccinees of study I by qRT-PCR longitudinally post challenge...

Figure 3-9: $\quad$ Calpox viral DNA in blood of vaccinees of study II by qRT-PCR longitudinally post challenge

Figure 3-10: $\quad$ Replicating calpox virus in blood of animals from vaccine study II

Figure 3-11: $\quad$ Levels of vaccine-induced binding antibodies were not associated with protection in vaccination study $\mathrm{I}$.

Figure 3-12: $\quad$ Levels of vaccine-induced binding antibodies were not associated with protection in vaccination study II.

Figure 3-13: $\quad$ Results for vaccination study I and II revealed no differences in binding antibodies between vaccination groups by ELISA at day of challenge....... 80

Figure 3-14: $\quad$ Results for vaccination study I and II reveal no significant differences in binding antibodies determined by ELISA between vaccination groups at challenge

Figure 3-15: $\quad$ ELISA antibodies against viral particles of calpox virus at the day of challenge virus exposure

Figure 3-16: Neutralizing antibodies against VACV LE and calpox virus in animals of vaccination study I at time of challenge

Figure 3-17: Titres of neutralizing antibodies per vaccination group in vaccination study I.

Figure 3-18: $\quad$ Neutralizating antibody titres in animals of vaccination study II 85

Figure 3-19: $\quad$ Mean \pm SD of the titres of neutralization antibodies per vaccination group for vaccination study II . .

Figure 3-20: $\quad$ Absolute cell numbers of $\mathrm{CD} 14^{+}$cells (monocytes) in vaccination study II are shown over time.

Figure 3-21: $\quad$ Absolute cell numbers of myeloid dendritic cells (mDCs) in vaccination study II are shown over time....

Figure 3-22: $\quad$ Absolute cell numbers of plasmacytoid dendritic cells (pDCs) in vaccination study II are shown over time.

Figure 3-23: Absolute cell numbers of NK cells in vaccination study II are shown over time

Figure 3-24: $\quad$ Absolute $\gamma \delta$ T cells in vaccination study II are shown over time

Figure 3-25: $\quad$ Absolute cell numbers of $\mathrm{CD}^{+} \mathrm{T}$ cells in vaccination study II are shown over time

Figure 3-26: Absolute cell numbers of $\mathrm{CD}^{+}$cells in vaccination study II are shown over time 90

Figure 3-27: $\quad$ Absolute cell numbers of $\mathrm{CD}^{+} \mathrm{B}$ cells in vaccination study II are shown over time

Figure 3-28: $\quad$ Absolute cell numbers of $\mathrm{CD}^{+} \mathrm{CD}^{+} \mathrm{B}$ cells in vaccination study II are shown over time. 
Figure 3-29: Absolute cell numbers of $\mathrm{CD} 20^{+} \mathrm{B}$ cells in vaccination study II are shown over time

Figure 3-30: $\quad$ Percentages of $\mathrm{CD} 86^{+}$cells of $\mathrm{CD} 14^{++}$cells in vaccination study II are shown over time.

Figure 3-31: $\quad$ Percentages of $\mathrm{CD} 86^{+}$cells of $\mathrm{CD} 14^{+} \mathrm{CD} 16^{+}$cells in vaccination study II are shown over time

Figure 3-32: $\quad$ Percentages of $\mathrm{CD} 86^{+}$cells of $\mathrm{CD} 16^{+}$cells in vaccination study II are shown over time

Figure 3-33: $\quad$ Proliferating $\mathrm{CD}^{+}$and $\mathrm{CD}^{+} \mathrm{CD}^{+}$cells $\left(\mathrm{CFSE}^{-}\right)$frequencies were measured and increased at one time point (4-weeks post first immunization)

Figure 3-34: $\quad$ Frequencies of $\mathrm{CD}^{+} \mathrm{CD}^{+} \mathrm{CFSE}^{-}$and $\mathrm{CD}^{+} \mathrm{CD}^{+} \mathrm{CD}^{+} \mathrm{CFSE}^{-}$cells over time 96 


\section{List of tables}

Table 2-1: General laboratory equipments and consumables..................................... 40

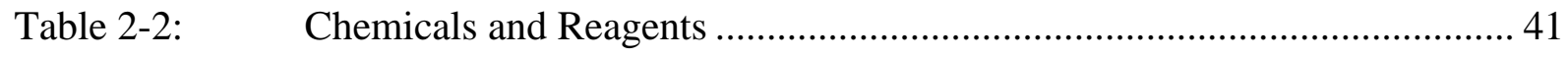

Table 2-3: $\quad$ Reaction components and commercial kits............................................... 42

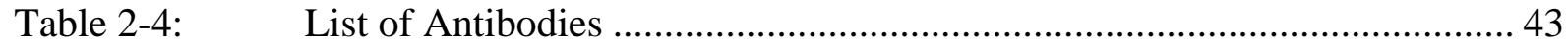

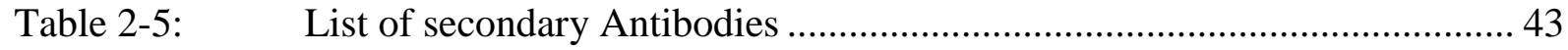

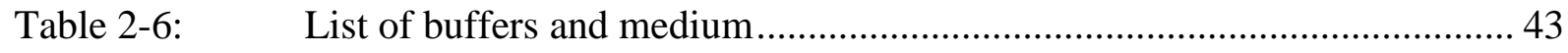

Table 2-7: $\quad$ Specification of common CM (Callithrix jacchus) used for the pathogenesis

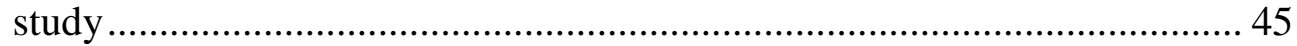

Table 2-8: $\quad$ Specification of common CM (Callithrix jacchus) used for the vaccination

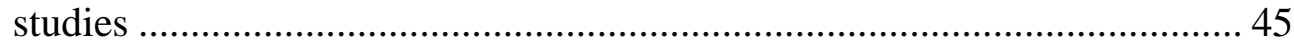

Table 2-9: $\quad$ Oligonucleotides used in calpox virus-specific real-time PCR assay.......... 60

Table 2-10: Cycling conditions and components for calpox virus-specific real-time PCR 60

Table 3-1: $\quad$ List of detected epitopes in protected animals after challenge .................... 82

Table 3-2: $\quad$ List of all mapped epitopes induced by vaccination ................................... 82

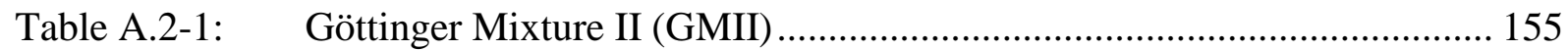

Table A.3-1: $\quad$ Detected replicating calpox virus in Callithrix jacchus individuals' blood for the pathogenesis study by viral plaque assay........................................... 155

Table A.3-2: $\quad$ Virus isolation of infectious calpox virus from buffy coat........................ 155

Table A.3-3: $\quad$ Overview of the detected antibodies in A27 in vaccination study I .......... 158

Table A.3-4: $\quad$ Overview of the detected antibodies in D8 in vaccination study I ............ 159

Table A.3-5: $\quad$ Overview of the detected antibodies in H3 and L1 in vaccination study I 160

Table A.3-6: Overview of the detected antibodies in A33 in vaccination study I .......... 161

Table A.3-7: $\quad$ Overview of the detected antibodies in B5 in vaccination study I ............ 162

Table A.3-8: $\quad$ Overview of the detected antibodies in B5 in vaccination study I ............ 163

Table A.3-9: $\quad$ Overview of the detected antibodies in A27 in vaccination study II......... 164

Table A.3-10: Overview of the detected antibodies in D8 in vaccination study II........... 165

Table A.3-11: Overview of the detected antibodies in H3 and L1 in vaccination study II166

Table A.3-12: Overview of the detected antibodies in A33 in vaccination study II......... 167

Table A.3-13: Overview of the detected antibodies in B5 in vaccination study II ........... 168

Table A.3-14: $\quad$ Overview of the detected antibodies in B5 in vaccination study II ........... 169 


\begin{abstract}
After the successful eradication of smallpox, mass vaccination stopped and the herd immunity against orthopoxvirus (OPXV) infections has been waning. In the light of bioterrorism, the interest in the development of antiviral drugs and safer vaccines against OPXVs increases. Therefore, a non-human primate model for OPXVs was established. Common CM (CM, Callithrix jacchus) are intranasally infected with a cowpox virus that is lethal for this nonhuman primate species and designated calpox virus.

A pathogenesis study was performed to determine the portal of viral entry and to analyze the early dissemination of the virus and the pathological sequelae. Groups of three animals were infected with either $3.5 \times 10^{5}$ plaque forming units (pfu) calpox virus and euthanized on day 3 , or with $8.3 \times 10^{3}$ pfu and euthanized on 5, 7, 10 and 12 days post infection (dpi). Blood and various organs were analyzed for infectious virus using the endpoint dilution assay and for viral DNA by real-time PCR. To detect the calpox virus infection in immune cells, PBMCs and buffy coat were analyzed by flow cytometry for calpox virus. Two vaccination studies aimed at identifying immune correlates of protection. Two attenuated smallpox vaccines, i.e. modified VACV Tian Tan (MVTT) and modified VACV Ankara (MVA) were tested for their efficacy after a 4- and 10-week waiting period. Humoral and cellular responses were analysed during immunization and after challenge, as well as viral DNA copy numbers and replicating virus after challenge.
\end{abstract}

Occurrance of viremia (DNA copies in blood) was dose dependent and already observed at 3 dpi (with the high inoculation dose) and after inoculation with the low viral dose at 7 dpi. The data suggests that the calpox virus initially replicated in the upper respiratory tract followed by systemic spread. In the prefinal phase, all organs became infected. Calpox antigen was detected in immune cells at different time points. With respect to the vaccine studies, overall, 67 $\%$ protection was observed following immunization with MVTT and $13 \%$ after MVA vaccination independently of the waiting period. All vaccine failures became virus positive. Virus-specific T-cell proliferation was observed in some animals vaccinated with MVTT. However, binding and neutralizing antibodies as well as the proliferative activity were not associated with the protection of the CM from calpox virus challenge. Peptide microarrays revealed antibodies against linear B cell epitope regions in different proteins (A33, B5 and L1) that were present almost exclusively in protected animals post challenge. Phenotyping of innate and adaptive immune cells by flow cytometric staining revealed no significant differences 
between vaccine groups in $\mathrm{T}$ and $\mathrm{B}$ cell numbers as well as in their expression of activation markers.

In conclusion, intranasal infection of $\mathrm{CM}$ with calpox virus led to a first local viral replication in nasal tissue. From there, the virus spread to various organs and in the prefinal phase all organs became infected. Highest protection was mediated by MVTT which therefore is superior to MVA in this model. So far, no obvious correlates of immune protection were identified.

Keywords: Calpox virus, pathogenesis, non-human primate model, calpox virus/marmoset model, smallpox vaccine, MVTT, MVA, New World 


\section{INTRODUCTION}

\subsection{Historical overview of orthopoxvirus infection}

\subsubsection{The smallpox virus}

\section{Origin of smallpox disease}

Smallpox is caused by the variola virus (VARV). The death rate of this devastating disease was estimated at $50 \%$ or more in young or over 40-years olds (Hanna and Baxby, 2002). Variola major and variola minor are the two known forms of the disease. Case-fatality rates between $5 \%$ and $40 \%$ were reported for variola major (Henderson and Moss, 1999). Variola minor occurs less often and is less severe than variola major with case-fatality rates between 1 $\%$ and $2 \%$. Unfortunately, a therapy has not been found until today. Smallpox spreads between humans without another animal reservoir.

Even though there is no evidence of the origin of smallpox, it is believed to have appeared in northeastern Africa at the time of the first agricultural settlement in 10,000 BC (Barquet and Domingo, 1997; Hopkins, 1983). It is assumed, that ancient Egyptian merchants were responsible for the spread of the disease from northeastern Africa to India (Riedel, 2005). The earliest reports of skin lesions resembling those of smallpox were found on faces of mummies of $18^{\text {th }}$ and $20^{\text {th }}$ Egytian Dynasties (1570-1085 BC). Evidence of the disease was found on the head of the mummy of the Egyptian pharaoh Ramses V, who died 1156 BC (Lyons and Petrucelli, 1987). Parallel to that, reports of a smallpox disease in ancient Asia appeared. In China, smallpox was described in ancient Sanskrit texts in 1122 BC. Smallpox first appeared in Europe between the $5^{\text {th }}$ and the $7^{\text {th }}$ centuries.

\section{History of variolation}

Material from smallpox-infected individuals was used in order to protect against pustules. During the $16^{\text {th }}$ century, Asians and Indians, inserted the fluid of smallpox scabs from an infected person into the nasal mucosa or carved into the skin of healthy persons (Needham, 1980). The method of treatment was called variolation and it caused a disease that was less severe than the one acquired via the respiratory system. Variolated patients were protected from death but still highly contagious and the risk of a new epidemic outbreak was high. This variolation was the first immunization method against smallpox (Variola) and was later introduced to Europe.

However, other diseases such as tuberculosis and syphilis were also transferred by variolation (Langer, 1976; Riedel, 2005). In the $18^{\text {th }}$ century, the method of immunization was replaced 
by the smallpox vaccine (Henderson, 2011). The smallpox vaccine introduced by Edward Jenner (Figure 1-1) was found to be safer. This vaccine was based on the finding that the maidservants, who developed cowpox lesions, were protected against poxvirus infections. In 1796, Jenner inoculated the eight year-old boy James Phipps with the material of a cowpox infection taken from the milkmaid Sarah Nelmes, and six weeks later with VARV (Paul, 2008). His experiment was successful and it produced complete protection against the disease and in 1798 Jenner described the safer and effective alternative to variolation. He named the infectious material of a cowpox infection "vaccine" after the Latin word "vacca" for cow. Although misleading, the English word "vaccination" is nowadays still in use (1.6.2 Cowpox viruses). Jenners discovery ranks among one of the greatest achievements in human history (Belongia and Naleway, 2003). His report on the usage of cowpox for vaccination against smallpox led to the confusion of the vaccinia virus (VACV) with cowpox virus (CPXV). When it was realized that the virus used for vaccination was not, or no longer the same as CPXV, the name 'vaccinia' was used for the virus in smallpox vaccine. Although the origin is unknown, it has been assumed that VACV was originally isolated from horses (Huygelen, 1996). Even if they were often considered to be the same, VACV and CPXV represent a distinct species among the genus Orthopoxvirus (OPXV) (Huygelen, 1996). Nevertheless, it was also reported that all available smallpox vaccines were based on VACV (Downie, 1939). It remains unclear whether Jenner originally used VACV or CPXV for his vaccination (Paran and Sutter, 2009).

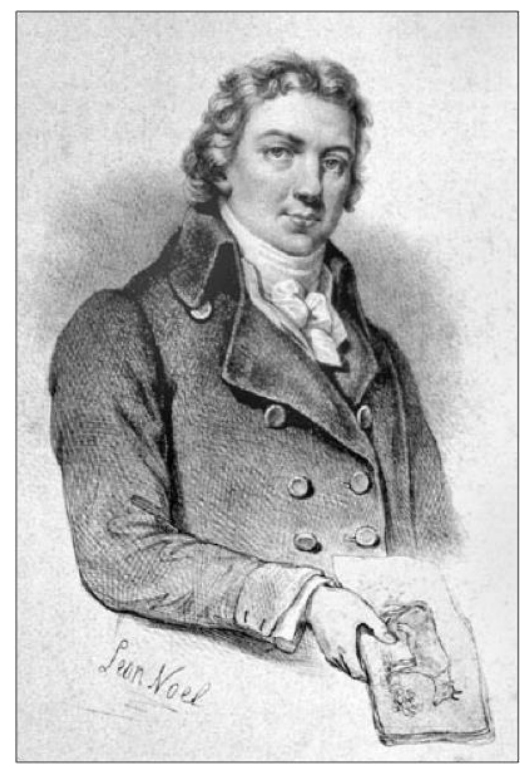

Figure 1-1: Edward Jenner (1749-1823) (Riedel, 2005). Jenner described the safer and more effective alternative "vaccination" to variolation in 1798. His discovery of the cross protection of cowpox and smallpox led to the eradication of smallpox as certified by the World Health Organization (WHO) in 1980 and is known as one of the outstanding achievements of medicine. Photo courtesy of the National Library of Medicine. 
After the discovery of the first vaccine consisting of the material of a cowpox infection, vaccinations were later performed by transmitting material from pustules of a vaccinated person to another individuum with the arm-to-arm method. This also brought the disadvantage of the accidential transmission of other diseases via blood. A further improvement for vaccine production was to grow the virus on calf skin. This method offered a safer and constant supply of vaccine material and increased the number of vaccinations in Europe (Moss and Henderson, 1999). A stable freeze-dried vaccine was commercially processed for large-scale production and established in the late 1940's (Collier, 1955). The smallpox vaccine was live vaccinia virus and belongs, like variola virus, to the OPXVs against which strongly cross-protective antibodies are induced after an infection (Essbauer et al., 2010; Gilchuk et al., 2016).

\section{Smallpox eradication}

Smallpox' lethal wanderings had an enormous influence on the human population (WHO, 1980). Five reigning monarchs died of smallpox in the eighteenth century in Europe. An estimated 400,000 Europeans were annually killed by the disease and by the end of the eighteenth century it was responsible for a third of blindness in Europe (Radetsky, 1999). Moreover, smallpox decimated Native American populations and thus facilitated the colonization of America (WHO, 1980). Except for Australia and on certain islands, smallpox was at its zenith endemic throughout the inhabited world (Buller and Palumbo, 1991). During the $20^{\text {th }}$ century, estimated 300-500 million people died from smallpox infections before a global smallpox vaccination campaign was initiated (Smith and McFadden, 2002). Despite of the discovery of the vaccination, many people died from the disease worldwide.

Following the decision of the Pan American Sanitary Organization in 1950, an eradication program was implemented. With the exception of Brasil, Argentina, Colombia and Ecuador, the attempt to eliminate smallpox in 1967 was successful in the Americas. The World Health Organization (WHO) initiated a global eradication program in 1959. The last naturally occuring case of smallpox was diagnosed in the town of Merca, Somalia in 1977 and the eradication of smallpox was certified by the WHO on May 8, 1980 (Figure 1-2) (Deria et al., 1980; WHO, 1980). 


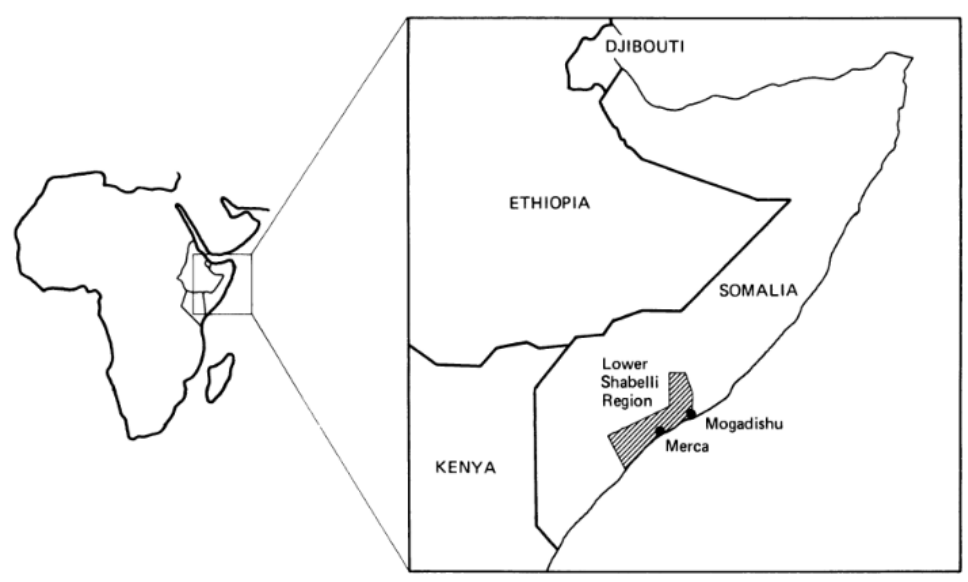

Figure 1-2: Location of the world's last known endemic case of smallpox (Deria et al., 1980). The last naturally occurring case of smallpox was diagnosed in the town of Merca, Somalia.

Because there was no effective animal reservoir for VARV, the global immunization program with the vaccinia virus was successful in eradicating this disease (Buller and Palumbo, 1991). Millions of lives were saved and the eradication of smallpox remains one of the outstanding medical achievements (Moss, 2011). In the case of smallpox, a virus was eradicated for the first and only time in human history. Officially, the variola virus is nowadays stored at only two institutes, the Vector Insitute in Novosibirsk, Russia and the Center for Disease Control and Prevention (CDC), USA.

Many different strains of VACV have been used for vaccination in the last two centuries (1.9.1 Vaccinia virus (VACV)). Due to the lack of information (origin, history of serial passage) about these strains, the origin of the present VACV strains is unknown.

\subsubsection{The potential threat of bioterrorism and zoonotic events}

In order to avoid severe side effects, vaccination with VACV stopped soon after the successful eradication of smallpox in 1980. Therefore, herd immunity is no longer given. If reintroduced, the human population remains unprotected. Routine smallpox vaccination ended in Europe between 1976 and 1984 (Fenner et al., 1988c). Third generation smallpox vaccines do exist, but the main purpose of these vaccines is for the protection of the military personnel and selected laboratories that work with OPXVs.

Because of the careful and exclusive storage of the virus, a voluntary or accidentially reintroduction of VARV is less likely yet existent and VARV pose a potential thread. However, the existence of other unregistered VARV stocks cannot be excluded. In June 2014, tubes labeled as "variola" were found in an unsecured freezer of a laboratory in Bethesda (Reardon, 2014). Furthermore, bioterrorist attacks, such as anthrax attacks in 2001, have sensitized the population and the awareness of the possible misuse of viruses in this context is ever present. 
Biological weapons, including VARV, have accidentially been used or exposed in planned tests. Smallpox is included in the category A of the CDC list of potential biological weapons (CDC, 2008) because of VARV's easy airborne transmission from one person to another as well as the high mortality rate and the low or not existing smallpox immunity in the majority of the human population (Wenzel, 2002). The re-introduction of VARV through a bioterrorist attack poses one of the greatest threats (Callaway, 2008; Henderson, 1999; Henderson et al., 1999; Jahrling et al., 2005; Kortepeter and Parker, 1999). Because of the risk of accidential or voluntary reintroduction through bioterrorism, the constant monitoring of smallpox must continue (Morand et al., 2017).

\subsection{Orthopoxvirus diseases}

\subsubsection{Virus transmission}

VARV is mainly transmitted through the respiratory route via virus containing droplets (Kempe et al., 1969), but infections via the conjunctiva have also been reported (Rao et al., 1960). Epidemiological studies have shown that the presence of VARV in scabs do not play a noteworthy role in virus transmission. A fetus can presumably be infected in the placenta during the second viremic phase of the mother (Marsden, 1936). No reports for infections via the alimentary tract or that of the involvement of arthropods exist.

Monkeypoxvirus (MPXV) is a zoonotic poxvirus (Essbauer et al., 2004) and the virus is therefore transmitted to humans through contact with wild animals that includes infected nonhuman primates (NHPs). The transmission rate increased from $30 \%$ in 1988 (Fenner et al., 1988a) to $73 \%$ in 2008 (WHO, 1980). Transmission chains beyond secondary infection have been reported (Hutin et al., 2001; Learned et al., 2005), but are rare (Jezek et al., 1986; Jezek et al., 1988). Therefore, it is assumed that MPXV infections only persists in the human population, if it is repeatedly re-introduced by the main reservoir (Fine et al., 1988).

Unlike VARV that is mainly airborne-transmitted, CPXV is another zoonotic poxvirus that is mainly transmitted to humans via skin or mucosal lesions. Most of the time it derives from scratches or bites of infected cats or pet rats leading to skin lesions commonly on fingers and hands. Virus-transfer from these lesions can later infect the face. CPXV infections transmitted by pet rats or other pet or zoo animals are increasingly reported and the number of animal species that are susceptible to CPXV infections increase similarily (Figure 1-3) (Baxby et al., 1979; Baxby et al., 1994; Becker et al., 2009; Campe et al., 2009; Kurth et al., 2009; MätzRensing et al., 2012; Pilaski et al., 1986; Schupp et al., 2011; Vorou et al., 2008; Wolfs et al., 
2002). CPXV infections are usually self-limiting. Severe generalized infections can occur in atopic persons, who receive glucocorticoids for therapeutic reasons or immunsuppressed HIV-patients (Blackford et al., 1993; Czerny et al., 1991; Klingebiel et al., 1988).

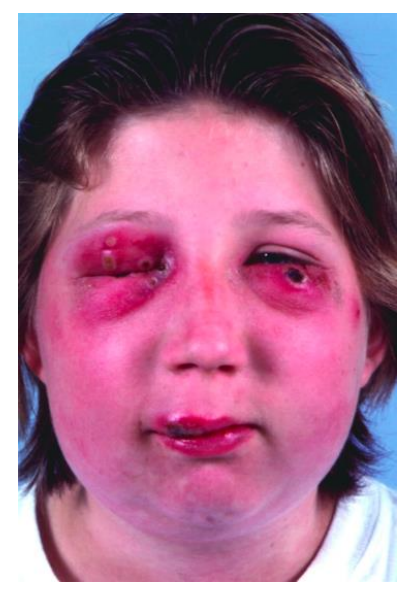

Figure 1-3: First proven case of rat-to-human transmission of cowpox in 2002 (Wolfs et al., 2002). Zoonotic infection of a 14-year-old girl who got infected (eyelid) after contact with a clinically ill wild rat that later succumbed to its infection.

\subsubsection{Clinical symptoms}

The most common clinical form of smallpox was the ordinary type and was caused by variola major and minor. Other clinical forms of smallpox were the modified type, the flat type and the hemorrhagic type where the last two types were usually fatal. Clinical symptoms of the ordinary type occur after an incubation period of approximately 12 days with a range from 7 to 17 days. The symptoms include headache, backache, high fever, malaise, prostration, and vomiting in rare cases that is followed by a maculopapular rash lasting for two to five days. The maculopapular rash develops to papules, vesicles and then to pustules. Most of the pustules are found on the soles of the foot, the face, the forearms, the palms of the hands, the mouth and the neck (Moore et al., 2006). Crusts start to form on day eight or nine. When they fall off, the skin underneath is pigment-free and remains scarred. Perhaps because of the developing antibodies, the fever sinks with the development of the rash and rises again with the development of the pustules. Conjunctivitis, which often leads to blindness in the patient, appears during the first eight days. The severeness of the disease is dependent on the immune response of the host and the cause of death is still not clear. The reason might be intravascular coagulation, massive secretion of cytokines and a cytopathic effect caused by a strong virus replication in the tissue that end in multi-organ dysfunction (Bray and Buller, 2004; Downie et al., 1953; Martin, 2002). Variola major was generally more severe than variola minor with a higher death rate. 
Other clinical forms can be observed in CPXV infections. The first typical lesion appears as an inflamed macula that turns into a papule and a vesicle within 12 days. The vesicle begins to ulcerate and becomes increasingly hemorrhagic. At the end of the second week the vesicle becomes incrusted (Dugmore and Dabir, 1992; Simcock et al., 1993). The degree of inflammation of the lesions varies, whilst the size of the extremely painful lesions ranges from one to three centimetres in diameter. An often marked and prolonged lymphadenopathy occurs. Systemic symptoms, such as fever, malaise, lethargy, in some cases vomiting and sore throat that lasts three to ten days, have been reported. It takes ten to 12 weeks or longer for the patient to fully recover. Ocular infections of the conjunctiva and cornea have occasionally been reported in localized CPXV infections (Hall and Stevens, 1987; Klingebiel et al., 1988). In more serious generalized infections, symptoms, such as atopic eczema, dermatitis, allergic asthma or immunosuppression, can occur and have a severe or lethal progression (Blackford et al., 1993; Czerny et al., 1991; Klingebiel et al., 1988).

\subsubsection{Pathogenesis}

Most of the information on the pathogenesis of VARV infections stem from previous smallpox cases that originated from artificial infections of animals with different OPXV (Buller and Palumbo, 1991; Hahon and Wilson, 1960; Zaucha et al., 2001). VARV initally infects the mucosa and the upper respiratory tract, the mucosal membranes of the mouth, the nasal cavity, the oropharynx or the alveoli of the lungs. After local replication, the virus then spreads through infected macrophages via the lymph system to the regional lymph nodes, in which replication continues. B cell and cytotoxic $\mathrm{T}$ cell responses are induced in the regional lymph nodes. The following virus replication leads to a first asymptomatic viremia between day five and six post infection (Fenner et al., 1988a). The virus is then disseminated to many organs (e.g. spleen, bone marrow, lymph nodes). After the invasion of the mononuclear phagocyte system, a short second viremia follows and VARV is characterised by the entry of the virus into the bloodstream at day eight post infection. The virus completely disseminates throughout the body and also the blood vessels of the dermis, the oral and pharyngeal mucosa in second viremia. Because of infected macrophages, VARV is spread from small dermal vessels to the epidermis. This leads to the typical pox lesions in the epidermis that occur through the development of edema, ballooning degeneration and splitting of the epidermis. The typical lesions (maculopapular, vesicular and papular rash) occur between days ten and 14 post infection. The infection starts with the occurance of the skin lesions. Skin lesions usually occur as soon as enanthems, mucosal lesions in the mouth and oropharynx occur. Fever and also cyto- 
kine secretion/cytokine storm occur during the second viremia. In addition, several Guarnieri bodies and extensive necrosis of epithelial cells of infected skin tissue can be detected through histological analyses. In hemorragic cases of VARV infections, the virus is only detectable after the death of the infected person in the pox lesions, spleen, kidney, liver, bone marrow and also other organs (Fenner et al., 1988a; Fenner et al., 1988b; Martin, 2002). Main source of virus transmission is oropharyngeal secret (saliva) that contaminates objects and can be easily transmitted via droplets. In some cases, the virus was also detected in urine. The contagiosity is highest in the first week after occurance of the skin lesions (Mac et al., 1950). Intravascular coagulation, massive secretion of cytokines that is associated with circulating immune complexes, soluble VARV antigens and a cytopathic effect based on massive virus replication in the tissues ending in multi-organ dysfunction have been discussed as the cause of death (Bray and Buller, 2004; Downie et al., 1953; Martin, 2002). Nevertheless, it remains unclear why individuals die.

\subsubsection{Calpox virus}

The studies presented herein are based on the findings of a private keeping of common CM (CM, Callithrix jacchus) in 2002 (Matz-Rensing et al., 2006). The animals developed a severe systemic disease with hemorrhagic lesions that resembled human smallpox, fever, depression, severe erosive-ulcerative lesions of the oral membranes and lymphadenopathy (Schmitt et al., 2014). The infected animals of a group of 30 to 80 New World monkeys died within one week after the onset of symptoms of the unknown disease. Mätz-Rensing and colleagues have found a new virus. The not yet fully characterized virus was named after its host Callithrix jacchus, calpox virus and was used to develop a promising OPXV model with CM. The virus was sequenced and identified to be the closest related to cowpox viruses. Nevertheless, calpox virus differs from the already described species and cannot be assigned to a previously known cowpox species (Carroll et al., 2011). It therefore represents a distinct and new CPXV strain.

In order to identify the cause of the animals' death, several pathological and histological analyzes and investigations in the CM followed (Kramski et al., 2010; Mätz-Rensing et al., 2012). Previously conducted studies focused on the final stage of the disease. Mätz-Rensing and colleagues detected the characteristic pox-like lesions in skin, mucous membranes, lymph nodes, liver and spleen. In parallel, Schmitt and colleagues experimentally infected CM with the calpox virus and analyzed different organs and tissues by histopathology, immunohistochemistry and transmission electron microscopy (Schmitt et al., 2017). The data indicates an 
early replication in nasal and bronchial epithelia as well as secondary replication in submandicular lymph nodes and spleen. A monocytic-cell associated viremia is followed by the detection of the virus in mainly epithelial cells and macrophages, but also in endothelial cells. The incubation period lasts about 12 days and resembles that of human smallpox. The definition is based on the histological and ultrastructural lesions, the onset of clinical symptoms and the immunohistochemical distribution pattern of the virus. The immune system obviously collapsed around day 12 post infection and animals start to die. Kramski and colleagues also found out that intranasal inoculation with very low infectious doses of the calpox virus leads to a severe, fatal disease course (Kramski et al., 2010). The intranasal inoculation route resembles the natural transmission route of smallpox.

\subsection{Orthopoxviruses within the Poxviridae family}

Two subfamilies belong to the Poxviridae family, the Chordopoxvirinae and Entomopoxvirinae. Entomopoxvirinae infect insects, whilst Chordopoxviridae infect vertebrates (Buller and Palumbo, 1991). Orthopoxvirus belongs to the Chordopoxvirinae and is one of the four genera of the poxviruses, notably Orthopox, Parapox, Yatapox and Molluscipox that may infect humans. The genera Orthopoxvirus (OPXV) with the type species vaccinia virus can be subdivided into ten species: Camelpox virus, Cowpox virus, Ectromelia virus, Monkeypox virus, Raccoonpox virus, Skunkpox virus, Vaccinia virus, Variola virus and Volepox virus (International Committee on Taxonomy of Viruses (ICTV), 2017). All OPXV species are serologically very closely related and therefore cross-reactive.

\subsection{Virus structure and genome}

With a size of $200 \mathrm{~nm}$ in diameter and $300 \mathrm{~nm}$ in length, the poxvirus virions are considered to be exceptionally large (Figure 1-4). The poxviridae viral particles (virions) are enveloped and generally brick shaped and carry the genome in a single, double-stranded DNA segment. The genome lies within a nucleoprotein complex in a biconcave capsid. In VACV, four different types of virions are produced in an infected cell: the extracellular enveloped virus (EEV), the intracellular enveloped virus (IEV), the cell-associated enveloped virus (CEV) and the intracellular mature virus (IMV). The virus particles differ in morphology, proteins and numbers of membrane layers. The enveloped virions (EV) have two membranes and are formed as long as the cell is still intact. After cell lysis, mainly mature virions (MV) are set free (Condit et al., 2006). Within CPXV- and ectromelia virus (ECTV)-infected cells also acidophilic-type inclusion bodies (ATI), or A-type inclusion bodies are formed and can be 
found in the cytoplasm (Patel et al., 1986). At the replication site (infected epithelial cells) Btype inclusion bodies or Guarnieri bodies are found, also virus particles aggregate during the infection.

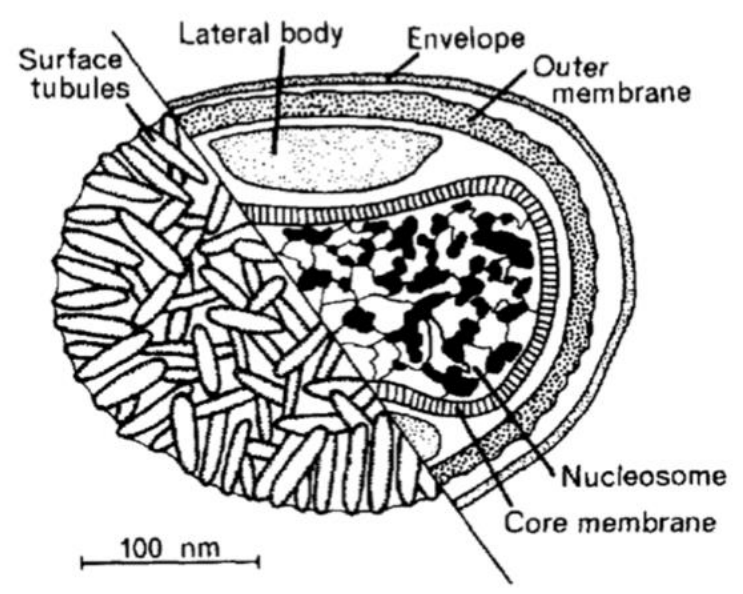

Figure 1-4: Drawing of extracellular enveloped vaccinia virus particle (Fenner et al., 1988a). Orthopoxviruses are remarkable large.

OPXV have a linear double stranded, linear DNA-genome of 180-190 kb in length and encode over 250 genes (Craighead, 2000). The ends are coupled by covalent hairpin structures (VARV) (Shchelkunov et al., 2000). OPXV replicate in the cytoplasm. Since replication machinery of the host is located in the nucleus and cannot be used by the virus, poxviruses encode for the complete functions of the replication cycle including polymerases, RNA-capping and RNA-modifying enzymes. The genomes of the Chordopoxvirinae are highly homologous. In the center of the genome there are 90 conserved genes that encode proteins for basal functions such as replication, transcription, virion assembly and release (Upton et al., 2003). The non-conserved genes are located at the ends of the genome and are species specific (Gubser et al., 2004; Shchelkunov et al., 1998). They determine the pathogenicity of the respective virus and encode for host range-factors and immunomodulators. Inverted terminal repetitions are located at the two ends of the OPXV genome (Garon et al., 1978). They consist of an AT-rich base-paired hairpin loop that connects the two DNA strands. The inverted terminal repetitions are variable in length but have a highly conserved region that is necessary for the processing of replicating concatemeric forms of DNA (DeLange and McFadden 1990; Merchlinsky 1990). 


\subsection{Virus life cycle}

\subsubsection{Virus entry and uncoating}

A ubiquitous receptor or the use of many cellular receptors has been assumed for OPXVs ability to infect a variety of cells. Although no specific cellular receptor has been detected for any of the OPXVs, glycosaminoglycans (GAGs) are thought to fulfill this function. The virus then enters the cell and uncoats in two steps. In the first step the outer membrane is removed when the virus enters the cell. In the second step the virus particle fuses with the cellular membrane and releases the virus core into the cytoplasm to express the virual genes (1.5.2 Virus gene expression). Furthermore, different virus particle subtypes probably enter the cells via different routes. It has been suggested that EEVs enter the cell via endocytosis. It is assumed that the low $\mathrm{pH}$ disrupts the virions outer membrane and released IMV fuses with endosomal membranes (Doms et al., 1990; Vanderplasschen et al., 1998). It is also assumed that vesicles are formed by surface invaginations (Gong et al., 1990; Kochan et al., 2008; Senkevich and Moss, 2005; Townsley et al., 2005; Vazquez et al., 1998).

There have been evidence that IMV and EEV have different binding sites (Vanderplasschen and Smith, 1997). When two types of virus bind to a cell, efficacy of infection may increase. Different proteins are involved in the cell attachment of EEVs (Chung et al., 1998a; Hsiao et al., 1998; Lin et al., 2000).

\subsubsection{Virus gene expression}

Expression of early-, intermediate-, and late-phase genes takes place in the cytoplasm of the host cell (Broyles, 2003). Viral RNA-polymerase begins expression of early-phase genes at approximately 30 minutes post-infection. This process starts inside the viral core which in the meantime becomes completely uncoated. At the time the early expression ends, the viral genome is released into the cytoplasm. Approximately half of all OPXVs genes belong to the early class (Oda and Joklik, 1967). The early mRNAs encode factors and enzymes that are needed for host interactions, viral DNA synthesis and transcription of the intermediate phase genes (Baldick and Moss, 1993). After early gene transcription, intermediate genes are expressed in the cytoplasm. At this time point, approximately 2 hours post infection, genomic DNA replication is triggered by viral DNA polymerase (1.5.3 DNA replication).

The intermediate genes are expressed from 140 minutes to 48 hours post infection and encode structural proteins to form a new mature virus particle (1.5.4 Virion assembly, maturation and release). 


\subsubsection{DNA replication}

DNA replication by viral DNA polymerase takes place entirely in the cytoplasm of the infected host cell and involves several stages (Broyles, 2003). DNA-replication occurs in discrete cytoplasmic structures known as virus factories or virosome (foci of replication, termed virus factor areas or Guarnieri bodies). The two strands of the linear, double-stranded DNA are linked by hairpin ends. Several viral proteins are involved in DNA synthesis: a 117-kDa DNA polymerase, a helicase-primase, an uracil DNA glycosylase, a progressivity factor, a singlestranded DNA-binding protein, a protein kinase and a DNA ligase (Moss, 2013). Replication begins with introduction of a nick in one of the viral DNA strands near the terminal region creating a free $3{ }^{\prime} \mathrm{OH}$ primer. This leads to strand displacement and elongation of the newly formed 3'end by the viral DNA polymerase. The elongated DNA strand possesses selfcomplementarity which allows it to fold back on itself and prime further elongation. This elongation can progress through the hairpin terminus. The replicated DNA appears as headto-tail concatemers, which could form by a rolling circle mechanism. In VACV about 10,000 genome copies per cell are generated by DNA replication and half of them are packaged into new virions (Joklik and Becker, 1964).

\subsubsection{Virion assembly, maturation and release}

The assembly of the virus particles is a complex process consisting of five stages and occurs in the cytoplasm of the cell. The assembly and virus morphogenesis occur together with the transcription of the late genes and genome replication in the viral factories (Rodriguez et al., 1997). Crescent membranes appear within the factories as first distinct structures of virus morphogenesis (Moss, 2015). It has been suggested that the crescent membrane is derived from the endoplasmatic reticulum. Crescents grow until they become three dimensional spheres to form the immature virion (IV). While crescent membranes develop, they become filled with viroplasm containing viral core proteins. Before the membrane is sealed, viral genomic DNA is packed into the viroplasm. The detailed mechanism of how the viral DNA is transported and incorporated into the virion remains unclear. VACV regulatory protein E6 is known to be essential for core protein association. By condensing into the dense brick-shaped mature virions (MVs), the IVs then maturate into intracellular mature virus (IMV), the infectious particles (Gaylord and Melnick, 1953; Morgan et al., 1954; Morgan and Wyckoff, 1950). The virus core condensates in this step and the major structural proteins enter the immature envelopes by proteolytic processing (Morgan, 1976; Moss and Rosenblum, 1973). Depending on the poxvirus genus, IMVs/MVs can also remain within the cell or may be 
wrapped into an additional membrane (Hiller and Weber, 1985; Schmelz et al., 1994; Tooze et al., 1993). The wrapped virions or intracellular enveloped viruses are transported to the cell periphery and in the final stage of maturation, the new extracellular enveloped virions are formed by exocytosis and exit the cell (Smith and Law, 2004). By losing the outermost Golgiderived membrane, the extracellular enveloped virions and cell-associated enveloped virions are formed. Cell-associated enveloped virions are important for cell-to-cell spread and remain attached at the cell surface. EEVs mediate the long-range dissemination of the virus in cell culture (Smith et al., 2002).

\subsection{Orthopoxviruses in humans}

\subsubsection{Zoonotic poxvirus infections in humans}

Humans and other primates are susceptible to OPXV infections. Five OPXVs can infect humans: variola, vaccinia, cowpox, monkeypox and camelpox.

One of the most devastating OPXV diseases in humans is known as the infection with VARV, the etiologic agent of smallpox (1.1.1 The smallpox virus). It has been assumed that VARV may have evolved from an enzootic pathogen of African rodents that spread from Africa, but no evidence exists (Li et al., 2007). Therefore, smallpox might have originally been acquired by humans as a zoonosis, from a terrestrial African rodent 16,000 to 68,000 years ago. However, smallpox was spread from human to humans without another animal reservoir.

Monkeypox, vaccinia, camelpox as well as cowpox are zoonotic (Goyal et al., 2013) and therefore regarded as infectious diseases of animals that can be transmitted to humans. Vacciniation of humans with live VACV against smallpox stopped soon after its eradication and since the waning herd immunity in the human population, other OPXV infections such as MPXV, CPXV or VACV in humans increased.

Worldwide MPXV and CPXV are sporadically occuring and humans are more often affected, especially by MPXV in Africa and CPXV in Europe, respectively (Bourquain et al., 2013; McCollum et al., 2015; Reed, 2004; Vorou et al., 2008).

MPXV-infection naturally occurs in Africa and particularly in the Democratic Republic of the Congo (DRC). MPXV is a zoonotic OPXV and an emerging infectious disease that causes serious smallpox-like illness in humans (Figure 1-5) (Rimoin et al., 2010; Thomassen et al., 2013). MPXV is endemic to West- and Central Africa since the 1970s (Nalca et al., 2005; Orba et al., 2015) and has been considered to be the most important poxvirus that affects human health since the global eradication of smallpox in 1977 (Breman, 2000). During the last 
30 years, human MPXV incidents have dramatically inreased in the DRC (Johnston et al., 2015). A comparison of active surveillance data from 1980s and 2006-07 suggest a 20-fold increase in human monkeypox incidents in the central DRC (Rimoin et al., 2010).

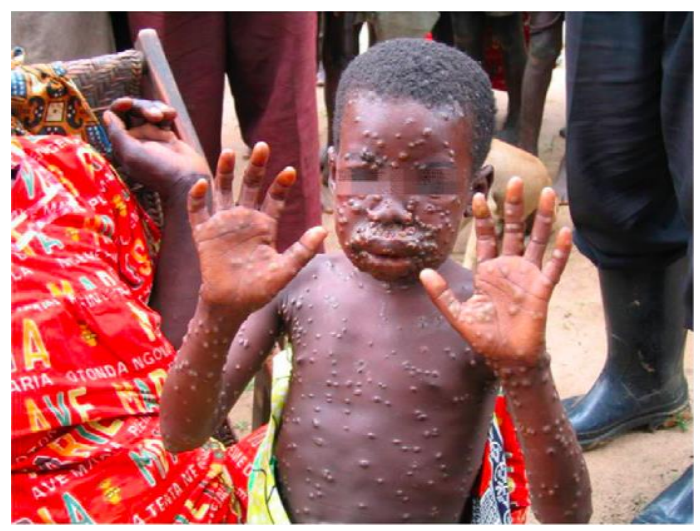

Figure 1-5: 7-year-old female child with the typical clinical presentation of human monkeypox, Sankuru District, Democratic Republic of Congo (Rimoin et al., 2010). Human monkeypox incidences increase 30 years after smallpox vaccination ceased in the Democratic Republic of Congo.

Another MPXV case shows that it was even able to spread to another continent. In 2003 a mild form of MPXV infected 69 people in the United States of America. Rodents and infected prairie dogs were imported from Ghana and transmitted the disease to humans. It can be assumed that vaccines against poxviruses also protect against MPXV and therefore the development of new and safer vaccines becomes more important (Hammarlund et al., 2005).

VACV occurs worldwide and infects a variety of host species such as cattle, rabbits, pigs, humans and buffalos (Bhanuprakash et al., 2010; Essbauer et al., 2004; Singh et al., 2008; Singh et al., 2007; Singh et al., 2006; Yadav et al., 2010). Since 1960 innumerous VACV outbreaks have been documented in different regions of Brazil. Also, reports of VACV species transmitted to humans via cows or Indian buffaloes were reported in Brasil and India (Goyal et al., 2013; Pereira Oliveira et al., 2014).

The number of reported VACV infections in humans are on the rise in South America and India, but it is not clear whether VACV infections are actually increasing or if the reports have just recently begun.

Camelpox is a disease restricted to camels and its causative agent is the OPXV camelpox virus (CMLV). Except for Australia, the disease is enzootic in almost every region, where camels are reared and naturally occurs on the African and Asian continents (Balamurugan et al., 2013). Although it only rarely infects humans, Jezek and colleagues have shown that camelpox can be transmitted to humans and arthropods (Jezek et al., 1983). Therefore, it also belongs to the zoonotic diseases. The emergence of zoonotic camelpox outbreaks in India is a serious public health concern (Bera et al., 2011). Due to increasing reports of camelpox cases 
and outbreaks in camels, Camelpox was considered an emerging public health problem (Balamurugan et al., 2013).

Furthermore, the threat of new emerging zoonotic poxvirus species exists (Shchelkunov, 2013).

\subsubsection{Cowpox viruses}

CPXV which is closely related to VACV is indigenous to Great Britain, and both the traditional cowpox virus strain and slightly different strains of the so-called 'cowpox-like` viruses can be found in Europe (Baxby et al., 1979; Carroll et al., 2011; Fenner et al., 1988a) and adjacent Russian states. Phylogenetic analyzes have shown that the CPXV isolates form two major monophyletic clades (cowpox-like and vaccinia-like) (Carroll et al., 2011). It can further be split into five distinct monophyletic clusters. All German CPXV isolates are in the cowpox-like clade (group 1-4), a sister to camelpox virus (CMLV), taterapox virus (TATV) and variola virus (VARV), whereas the vaccinia-like clade (group 5) is a sister to MPXV clade.

Of all the OPXVs, CPXV has the broadest host range. Contradictory to its name, CPXV rarely occurs in cattle and it is doubtful if cowpox was ever common in cattle (Baxby, 1977). Human cowpox is uncommon, but more commonly reported than bovine CPXV cases (Baxby and Bennett, 1990). CPXV maintains a reservoir in wild rodents and is endemic in bank voles (Clethrionomys glareolus) and wood mice (Apodemus sylvaticus) in Great Britain (Chantrey et al., 1999). CPXV has also been reported to infect a variety of animals in European zoos, e.g. elephants that also resulted in human infections (Kurth et al., 2008). Although several carriers have been reported, it is mainly transmitted by cats and pet rats (Begon et al., 1999; Bonnekoh et al., 2008). Most reports of human cowpox infections can be traced back to contact with infected cats (Casemore et al., 1987; Pether et al., 1986; Willemse and Egberink, 1985). However, CPXV infections have also been reported in monkeys. A fatal cowpox virus infection in the cotton-top tamarins (Saguinus oedipus) a New World monkey, have been detected in Germany (Figure 1-6) (Kalthoff et al., 2014). A low pathogenicity was found when the isolate was characterized in a Wistar rat model. 


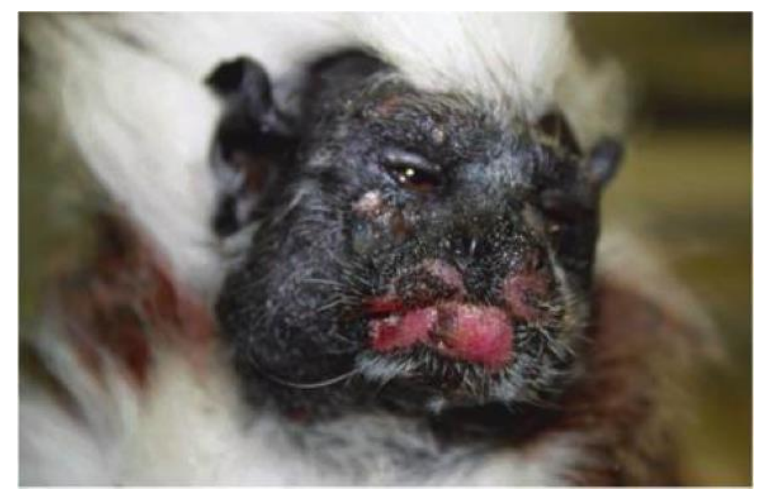

Figure 1-6: Cowpox virus-infected cotton-top tamarin (Kalthoff $\boldsymbol{e t}$ al., 2014). The New World monkey shows the typical skin lesions on the face.

Other CPXV infections in Germany and the Netherlands have also been reported in New World monkeys and macaques (Martina et al., 2006; Matz-Rensing et al., 2006) and a distinct and new CPXV strain, named calpox virus, was found in 2002 in common CM (CM, Callithrix jacchus) (Matz-Rensing et al., 2006) (1.2.4 Calpox virus).

Because of its broad host range and genetic features, CPXV is assumed as a candidate and threat for new emerging zoonotic poxvirus species (Essbauer et al., 2010). Of all OPXV, CPXV has the largest genetic repertoire (Gubser et al., 2004). This underlines CPXV as an emerging hazard.

\subsection{Immune response}

The severeness of the disease, clearance of the virus and protection against (re-)infection is determined by the immune responses of the host. Humoral as well as the cellular immune responses play an important role in OPXV infections (Smith and Kotwal, 2002). The immune system is differentiated into the innate and adaptive immune system. The innate immune system is fast and unspecific, whilst the adaptive immune system takes more time, but is specific and has memory.

\section{Innate immune response to poxvirus infection}

Following infection the immune system is activated, whereas the innate immune response constitutes the first line of defense against invading pathogens. At first, poxvirus infection induces the production of interferons as well as the activation of the complement system, natural killer (NK) cells and inflammatory cells (Smith and Kotwal, 2002). Monocytes and neutrophils are the most important parts in early innate immunity against poxvirus infection, because of their positive poxvirus antigen staining (Song et al., 2013b). Besides the cellular response, the humoral-mediated immunity (e.g. complement system) is of importance. The complement system has the ability to recognize a large panel of viruses as well as virus- 
infected cells, and trigger effector pathways (Agrawal et al., 2017). Interferons can e.g. kill the virus directly. The complement system marks the infected cells (opsonization) and consequently phagocytes can detect and kill them.

\section{Adaptive immune response to poxvirus infections - Cellular immune response}

The cellular immune response and the implied poxvirus-specific cytotoxic T-lymphocytes (CTLs) $\left(\mathrm{CD}^{+}\right)$are thought to play an important role in controlling poxvirus infections (Smith and Kotwal, 2002). $\mathrm{CD}^{+} \mathrm{T}$ cells detect early expressed proteins whilst $\mathrm{CD} 4^{+} \mathrm{T}$ cells, like antibodies, detect late expressed proteins (Sette et al., 2009). Experiments in knockout mice and macaques have shown that $\mathrm{T}$ cell response plays a more important role in the primary, acute infection than in the late phase (Gordon et al., 2011; Xu et al., 2004b). In addition, the development of severe vaccination complications, such as a progressive vaccinia in humans with an impaired cellular immunity, leads to the assumption that $\mathrm{T}$ cells play an important role in primary infections (Bray and Wright, 2003).

Poxviruses have a variety of immune evasion mechanisms to create an environment where the virus can not only survive but also replicate (Seet et al., 2003). There are two ways for the virus to interact with the host: the poorly understood inhibition of the host macromolecular synthesis by the virus and the defense of the virus against host antiviral mechanisms.

Several viral defense proteins are expressed by OPXVs in order to modulate and combat the host's antiviral response. They encode for proteins that induce the synthesis of steroids, inhibit the function of interferons, intercept interleukins and capture chemokines, inflammatory cytokines and antibodies. They also encode proteins that can interfere with the function of apoptosis. The activity of cytotoxic T-lymphocytes and NK cells can be inhibited and counteract the complement system (Alcami and Koszinowski, 2000; Haga and Bowie, 2005; Kotwal et al., 1990; Lustig et al., 2004; McFadden and Murphy, 2000; Perdiguero and Esteban, 2009; Taylor and Barry, 2006). Poxviruses can also shut down the synthesis of the cell's own products while keeping up their own protein synthesis (Langland et al., 2006). The immunomodulatory proteins are not shared across all OPXV species and each OPXV strain has its own combination of proteins. The deletion of some genes leads to the attenuation or an altered disease (Johnston and McFadden, 2004). Although each virus has its own variety of immunomodulatory proteins, some of the immunomodulatory viral genes are similar to cellular genes of the host. It is assumed that these genes were integrated into the viral genome via horizontal gene transfer through coevolution with the host. 
Poxviruses can harm the host through replication in the tissue, cause immunomodulation and furthermore, a strong reaction of the immune system can be triggered: an exessive inflammatory response (cytokine storm), which is discussed as the reason for many symptoms and for the cause of death of severe VARV-infections (Stanford et al., 2007). For instance, it has been reported that an intensive early inflammatory response by the host is thought to damage the host inadvertetly to such an extent that the individuum becomes ill or dies after the infection (Stanford et al., 2007).

\section{Adaptive immune response to poxvirus infections - Humoral immune response}

A human orthopoxvirus infection can elicit a complex B cell immune response that is reactive to antigens from diverse OPXV species (Gilchuk et al., 2016a; Gilchuk et al., 2016b).

Neutralising antibodies (nAbs) - as part of the humoral arm of the adaptive immune response - are most important because they bind directly to the poxvirus, cause aggregation and also prevent the adsorption and intake into the cell. They also enable phagocytosis and antibodydependent cell-mediated cytotoxycity through their function at opsonisation (process of marking the virus and ingestion or elimination by phagocytosis) (Edghill-Smith et al., 2005).

Results from animal studies indicated that neutralizing antibodies are essential and sufficient for an immunological protection (Edghill-Smith et al., 2005). Antibodies against specific OPXV-species have a wide cross-reactivity among the whole virus genus and depending on the antigenic relationship, they also have a wide cross-reactivity among the genus (Gilchuk et al., 2016a). NAbs are directed against gene products (Demkowicz et al., 1992) as well as different virus particles such as intracellular mature virions and enveloped virions generated during the infection (Amanna et al., 2006; Demkowicz et al., 1992; Jones-Trower et al., 2005; Viner and Isaacs, 2005).

In patients that suffered from non-hemorrhagic smallpox disease nAbs were detectable by day six of illness (about 18 days after infection). Titres increased over the course of the disease (Downie et al., 1969). A delayed, reduced or even missing antibody response was found in patients with a hemorrhagic-type of disease (Fenner et al., 1988c). NAbs were detected from day 12-14 and until day 25-30 in smallpox (Frey et al., 2003; McClain et al., 1997). Neutralizing antibodies are generated after 12-14 days when immunized with VACV and the highest titres are measured about two weeks later. After a re-vaccination the titres of nAbs increase already after seven days (Moss, 2011). Because of the eradication of VARV, it is difficult to calculate the protective effect of an immunization. Nevertheless, the antibody levels detected by ELISA or neutralization of VACV can persist for many decades as in memory B cells and 
$\mathrm{CD}^{+}$and CD8 ${ }^{+}$T cells (Crotty et al., 2003; el-Ad et al., 1990; Frey et al., 2003; Hammarlund et al., 2003; Taub et al., 2008). Studies have shown that in $90 \%$ of the observed individuals, a stable humoral immune response was detected up to 75 years after the immunization (Crotty et al., 2003; Hammarlund et al., 2003). It has also been observed that antibody titres were at a comparable level in immunized individuals and non-immunized individuals that survived VARV infections (Hammarlund et al., 2010; Taub et al., 2008).

Studies that induced the protection of macaques against monkeypox have suggested that nAbs are sufficient for protection (Edghill-Smith et al., 2005). Different proteins of the inner and outer membrane of the virus have been identified as neutralising determinants (Moss, 2011). It is supposed that nAbs against both enveloped viruses well as mature viruses are necessary for the protection against OPXV infections (Bray and Buller, 2004; Fogg et al., 2004; Heraud et al., 2006; Hooper et al., 2000; Lustig et al., 2005; Viner and Isaacs, 2005). The inoculation with VACV elicits nAbs against major antigens on the extracellular enveloped virus and the intracellular mature virion and confer protection against smallpox (Davies et al., 2005; Davies et al., 2007; Lawrence et al., 2007; Putz et al., 2006). The neutralising determinants A27, D8 and $\mathrm{H} 3$ are important for binding of the virus to the host cell on the inner membrane (Chung et al., 1998b; Hsiao et al., 1999). One protective antibody that binds to the heparan sulfate binding site of A27 was identified (Kaever et al., 2016). A17, L1 and A28 are involved in virus-cell membrane fusion (Nelson et al., 2008; Wallengren et al., 2001; Wolffe et al., 1995). A protective antibody response is induced by the protein B5 and A33 of the outer membrane, but only binding of antibodies to B5 has a neutralizing effect (Galmiche et al., 1999; Putz et al., 2006). The effect of neutralization of anti-B5-antibodies can be enhanced by the isotypespecific complement system (Benhnia et al., 2009). Antibodies against A33 also function in association with the complement system. By destroying the outer membrane of the virus particle, the inner membrane is set free and binding of anti-mature virus-antibodies is enabled (Lustig et al., 2004).

\subsection{Immune correlates of smallpox virus protection}

The smallpox eradication occurred prior to modern techniques in virology and immunology (Moss, 2011) and some live vaccines of VACV strain caused generalized VACV infections in patiens with severe $\mathrm{T}$ cell abnormalies, but not in others with an immunodeficiency (agammaglobulinemia). Therefore, evidence for the importance of a cell-mediated immunity in controlling the primary infection exists. Increased levels of cytokines correlated with low-grade fever, headache, myalgia, fatigue and regional lymphadenopathy after VACV infection. NAb 
titres to VACV correlated with the "vaccine take" (i.e. development of pustules) in naive individuals (Mc et al., 1958). In individuals who were already vaccinated and re-vaccinated correlated the successful vaccination inversely with the pre-existing nAb levels (Orr et al., 2004). Studies have shown that the route of vaccination is important. A higher $\mathrm{nAb}$ response was induced by percutaneous vaccination than with intradermal or intramuscular vaccination (McClain et al., 1997). Children developed $83 \%$ nAbs with percutaneous and $23 \%$ nAbs with subcutaneous vaccination (Galasso et al., 1977). Some studies have also found that smallpox vaccination induced $\mathrm{CD}^{+}$and $\mathrm{CD}^{+} \mathrm{T}$ cells (Ennis et al., 2002; Rock et al., 2005).

\subsection{Prevention (vaccine) and treatment of OPXV infection}

\subsubsection{Vaccinia virus (VACV)}

The origin of the vaccinia virus (VACV) remains unknown due to the lack of recording the repeatedly cultivating and passaging in research (Moss and Henderson, 1999). VACV is closely related to the cowpox virus (CPXV).

The disease pattern of a VACV infection closely resembles that of the local human CPXV infections (Paran and Sutter, 2009). Naïve individuals inoculated with VACV through vaccination or by accident, develop local infections of the eye or skin. However, VACV infections of at-risk individuals that are for example immunocompromised can lead to a severe disease or fatalities.

Vaccination with VACV comes with several advantages. Reports have shown that vaccination with VACV induces an antibody response that is developed much faster than after a natural VARV infection. Antibodies were detected ten days after primary vaccination and four to seven days after the second vaccination (Mc et al., 1958). The titres of neutralizing antibodies (nAbs) also increased as a reaction to the re-vaccination.

VACV strains have been used to establish more virulent laboratory viruses like VACV Western Reserve (WR) or VACV International Health Department-J (IHD-J) but have also been used for new vaccines. Various VACV strains were used as smallpox vaccines (McCollum and Damon, 2014; Verardi et al., 2012). The first generation smallpox vaccines were produced and used during the worldwide eradication program. Different VACV strains such as Lister-Elstree (Lister Institute, Elstree, England; propagated by the National Public Health Institute, Netherlands) (Fenner et al., 1988a), the New York City Board of Health strain (NYCBH e.g. Dryvax) (propagated by Wyeth Laboratories, USA) (Moss and Henderson, 1999), the Tiantan strain (Temple of Heaven) that was used in Asia (Zhang et al., 2013) and 
the EM-63 strain (NYCBOH) that was used in Russia (Fenner et al., 1988a) belongs to these first-generation smallpox vaccines. Because of the serious side effects, such as eczema vaccinatum, progressive vaccinia, generalized vaccinia, postvaccinal encephalopathy and encephalitis in some individuals, the production and licensing of these vaccines stopped in the late 1980’s.

The same smallpox vaccine strains employed for the manufacturing of first-generation vaccines were used for second-generation smallpox vaccines. These include the live attenuated smallpox vaccine ACAM2000 (NYCBOH; licensed in August 2007 by the FDA, USA) (Frey et al., 2007) or VACV LE-BN (Lister-Elstree strain produced by Bavarian Nordic, Martinsried, Germany) (Stittelaar et al., 2006; Uebler, 2008) produced in embryonic chicken fibroblast cells. Also, clonal virus variants plaque purified from traditional vaccine stocks were used. The second-generation vaccine strains were in vitro serially passaged in high numbers with sterile cultivation in cell culture with the purpose to increase attenuation and safety.

Third-generation smallpox vaccines are even more attenuated vaccine strains that were developed by further passage in cell culture or animals as safer vaccines at the end of the eradication. A safer third-generation smallpox vaccine is for example the replication competent LC16m8 (licensed in 1975, Japan) (Kennedy et al., 2011) that was developed from Lister strain. It was passaged in primary rabbit kidney cells and has a deletion in B5R. Another third-generation vaccine is the replication-deficient MVA strain Imvanex (also designated Imvamune or MVA-BN, licensed in July 2013 by the European Commission) (Frey et al., 2007; Garza et al., 2009) that was passaged on chicken embryo fibroblasts and its replication competency is limited in mammalian cell lines. Modified vaccinia virus Tiantan (MVTT) is a third-generation smallpox vaccine that was developed from the Asian Tiantan strain by removing the hemagglutinin gene. Its mucosal application protected mice against the VACV Western Reserve (WR) strain (Yu et al., 2010).

Fourth-generation smallpox vaccines represent non-infectious subunit vaccines that are composed of few viral antigens or proteins (Paran and Sutter, 2009). The proteins are expressed from DNA, recombinant viruses or replicons. The most frequently used proteins that were detected and used in combination are B5, L1, A33 and A27 (Aldaz-Carroll et al., 2005; Aldaz-Carroll et al., 2007; Fogg et al., 2004; Fogg et al., 2007; Golovkin et al., 2007; Heraud et al., 2006; Hooper et al., 2000; Hooper et al., 2004; Lustig et al., 2005; Xiao et al., 2007). They were effective in several animal models (e.g. non-human primates infected with MPXV) (Heraud et al., 2006). Fourth generation vaccines are for example a DNA vaccine that comprises a combination of IMV and EEV genes and core antigen and gave protection in rhesus 
macaques against monkeypox challenge (Hirao et al., 2011). Another experimental vaccine consisted of a combination of IMV and EEV proteins and also provided protection in animal models (Buchman et al., 2010). A multi T cell epitope vaccine based on conserved VACV and VARV sequences is another fourth generation vaccine (Moise et al., 2011). It gave protection in a mouse model.

\subsubsection{Modified vaccinia virus Ankara (MVA)}

The highly attenuated strain modified vaccinia virus Ankara (MVA) belongs to the third generation of vaccinia strains and was developed in the 1960s. It was attenuated by more than 570 passages in primary chicken embryo fibroblasts (CEFs). In comparison to the original strain, it contains six deletions that come with a $15 \%$ loss of genetic information (Antoine $e t$ al., 1998; Meyer et al., 1991). The high number of MVA passaging led to a strongly reduced replication competency on most mammalian cell lines and the virus only replicated in CEFs (Earl et al., 2004; Meyer et al., 1991). MVA was proven to be avirulent (Mayr et al., 1978). Several studies have confirmed the good tolerability of MVA and in the 1970s over 120,000 humans received primary vaccination in Germany (Hochstein-Mintzel et al., 1975; Mayr and Danner, 1978; Mayr and Danner, 1979). The ability of MVA to protect humans against VARV infections was not tested due to smallpox' eradication (Frey et al., 2007; Garza et al., 2009; Kennedy and Greenberg, 2009; Vollmar et al., 2006; von Krempelhuber et al., 2010). Studies later confirmed the good compatibility even in immunosuppressed individuals in different animal models (Cosma et al., 2003; Earl et al., 2004; Meyer et al., 1991; Stittelaar et al., 2001). MVA was also tested in an atopic mouse model and protective immunity against a lethal poxvirus challenge with VACV WR was proven (Knitlova et al., 2014).

The smallpox vaccine Imvamune (also Imvanex or MVA-Bavarian Nordic (BN) is a derivative of MVA. It differs to all other MVA strains in that it has undergone six rounds of plaque purification and is based on a single clone from the passage MVA-597 that was derived from MVA-584 (i.e. $584^{\text {th }}$ passage in CEF cells) and could induce an immune response in several studies. In several phase-II studies, Imvamune was tested to be safe in atopic and immunosuppressed individuals (Frey et al., 2007; Frey et al., 2013; Greenberg et al., 2013; von Krempelhuber et al., 2010; Walsh et al., 2013). However, Imvamune (also Imvanex or MVA $\mathrm{BN}$ ) was tested in the calpox virus/marmoset model and did not induce protection (Dr. StahlHennig and Dr. Mätz-Rensing, personal communication). Its suitability as smallpox vaccine is yet unclear. 
MVA (Imvamune and recombinant vaccinia virus) also gave solid protection in mice and macaques against MPXV (Hatch et al., 2013; Zielinski et al., 2010).

\subsubsection{Modified vaccinia virus Tiantan (MVTT)}

During the smallpox eradication campaign in 1960-1970s, vaccinia Tian Tan (VTT) was the most extensively used smallpox vaccine in China for millions of people beween 1920 and 1980 (Fang et al., 2005). The immunogenicity is good and the complications in humans are relatively mild. VTT is less virulent than VACV WR, but is still neurovirulent in mice and causes over $25 \%$ body weight loss after an intranasal inoculation. Therefore, the use as a noninvasive vaccine is restricted (Fang et al., 2005). The results showed that VTT should not be applied mucosally in humans and the need for further attenuation has been demonstrated (Fang et al., 2005). The virulence of VTT is of significant risk to children and immunocompromised adults (e.g. with HIV/AIDS, cancer, leukemia, lymphoma, multiple myeloma, etc) (Yu et al., 2010). As VTT was further attenuated through the deletion of inter alia, the hemagglutinin gene, leading to the generation of the modified vaccinia virus Tiantan (MVTT) (Yu et al., 2010; Zhu et al., 2007). The weight loss and neurovirulence in mice after intranasal inoculation was not observed in MVTT. Unlike VTT, MVTT did not replicate in mouse brain and was also safe in immunideficient mice (Yu et al., 2010). Protection was induced after an infection with VACV WR after one-time intranasal and intramuscular vaccination with MVTT in a mouse model (Yu et al., 2010). So far it has only been tested in the mouse model and therefore represents a promising approach to test the vaccine efficacy in a primate model (Huang et al., 2009; Zhu et al., 2007).

The needle-free mucosal application of MVTT was applied in the present studies (peroral and intranasal) and presents an easy alternative to intramuscular inoculation. It would also facilitate vaccination especially in developing countries (Meseda et al., 2005).

\subsubsection{Treatment of OPXV infections}

OPXV infections can be treated with ST-246, which is a specific inhibitor of EEV and provided $100 \%$ protection against a lethal infection of different OPXVs in different animal models (Grosenbach et al 2008, Quenelle et al 2007, Quenelle et al 2007, Yang et al 2005). It was also efficiently used in the treatment of a two-year old with a severe eczema vaccinatum (CDC 2008). Although therapeutics for OPXV infections do exist (e.g. ST-246) cidofovir is the only approved treatment of OPXV infection. Cidofovir is a nucleoside analog and has been used for the treatment of Cytomegalovirus infections (De Clercq 2002). There are sever- 
al compounds for OPXVs existent that were studied in cell culture or in animal models (Harrison et al 2004, Smee et al 2002, Smee and Sidwell 2003, Kern 2003, Byrd et al 2004, Damaso and Moussatche 1998, Quenelle et al 2006, Reis et al 2006, Su et al 2005). Other antiviral therapies like passive immunotherapy with anti-VACV immune serum (Berhanu et al 2008), siRNAs against potential OPXVs (Dave et al 2006) or DNA aptamers with antiviral activity (Nitsche et al 2007) were tested.

\subsection{The common marmoset - Callithrix jacchus}

The common marmoset (CM, Callithrix jacchus) belongs to the Callitrichidae family and is a New World monkey. They are originally found in the northeastern coast of Brazil, in the states of Piaui, Paraiba, Ceará, Rio Grande do Norte, Pernambuco, Agolas and Bahia. The monkeys are brown, grey and yellow and have white ear tufts. They also have a long, banded tail (Zhang et al., 1996). The males and females have a small size of 16 to $21 \mathrm{~cm}$ and a weight of 300 to $350 \mathrm{~g}$. Characteristic for this species are the claw-like nails (tegulaes) on their fingers except for their halluxes (big toes). This characteristic is necessary for their diet and arboreal lifestyle and is unique for this group. They live in small groups or in family organizations of about nine to 15 members with a flexible social structure and pairing system (Digby and Barreto, 1993; Rowe et al., 1996). Whilst most matings are monogamous, polygamy and polyandry have been observed in CM. CM commonly give birth to non-identical twins (Figure 1-7).

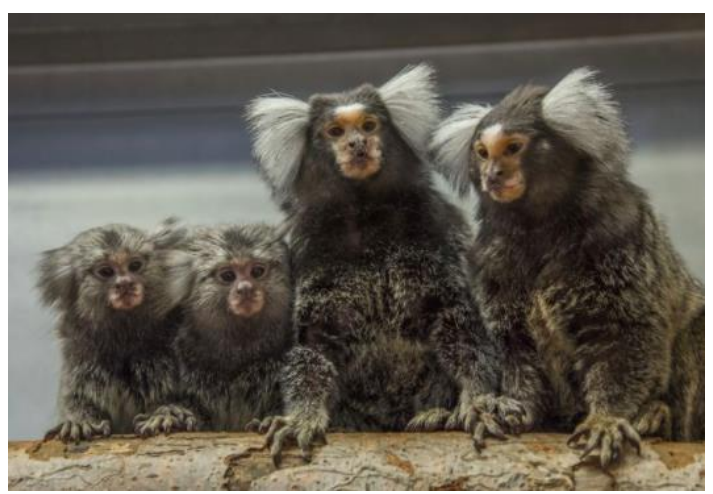

Figure 1-7: Two adult Callithix jacchus individuals with twins (www.dpz.eu, 2017). Adults showing the typical white ear tufts. Photo by Manfred Eberle.

The CM is exudativore-insectivore and feeds on plant exudates like gum, sap, latex and resin. They also feed on fruits, seeds, flowers, fungi, nectar, snails, lizards, tree frogs, bird eggs, nestlings and infant mammals. In the wild, CM can reach the age of 12 years (Rowe et al., 1996). New World monkeys are increasingly used in biomedical research (Abbott et al., 2003). 
We used the CM as in the non-human primate model because compared to Old World monkeys it is more convenient and has certain advantages, such as lower breeding and animal husbandry costs and an easier handling. The $\mathrm{CM}$ is also immunological related to humans but natural chimerism occurs (bone marrow chimerism) (Uccelli et al., 1997). CMs are used in several animal models for human diseases e.g. infectious diseases like anthrax (Carrion et al., 2007; Lever et al., 2008; Woollard et al., 2008), multiple sclerosis (Genain and Hauser, 1997; t Hart et al., 2000). Parkinson’s disease (Eslamboli, 2005; van Vliet et al., 2008), Alzheimer's disease, stroke (Marshall and Ridley, 2003) and immunotoxicological studies (Neubert et al., 1995) or spinal cord injury. Therefore, the CM represents a suitable model for studying vaccines and therapeutics.

\subsection{Animal models - a non-human primate model of OPXV infection}

Due to the fear of bioterrorism, and the increasing cases of MPXV and CPXV infections, research for therapeutics and prevention of OPXV infection is ongoing e.g. DNA vaccines (Hirao et al., 2011; Hooper et al., 2003; Pulford et al., 2004; Sakhatskyy et al., 2008), protein vaccines (Buchman et al., 2010; Davies et al., 2005; Fogg et al., 2004), T cell epitope vaccines (Moise et al., 2011; Puissant and Combadiere, 2006; Sakhatskyy et al., 2006; Verardi et al., 2012).

Beside mouse models, two well established NHP models exists: MPXV infection of cynomolgus macaques (Macaca fascicularis) (Hooper et al., 2004) and rhesus macaques (Macaca mulatta) as well as VARV-infected infection of cynomolgus macaques (Jahrling et al., 2004). So far, the best model for human smallpox is the MPXV-infected cynomolgus macaque model (Mucker et al., 2013). Because of the challenging handling with VARV (BSL-4), this model can only be used in BSL-4 laboratories. Howerver, working with MPXV (BSL-3) is limited. Compared to other, smaller animals, the macaques used in these studies are relatively expensive to keep. Also, a very high infectious dose via intravenous inoculation is necessary. Because of their close relation to humans these primates are the gold standard for OPXV models and for the testing of safer vaccines and antiviral drugs (Jordan and Hruby, 2006).

The CPXVs, calpox virus, was accidentally discovered (1.2.5 Calpox virus) and represents a suitable virus to infect common CM (CM, Callithrix jacchus). It induces a reproducibly lethal and systemic disease that leads to cutaneous pox lesions (Kramski et al., 2010). CPXVs have been used in other non-human primate (NHP) models (Johnson et al., 2015; Johnson et al., 2011) and the advantages and disadvantages of the here used calpox virus/marmoset model have been discussed in chapter 4.3.1 (4.3.1 Advantages and disadvantages of the calpox vi- 
rus/marmoset model). Schmitt and colleagues reviewed the different models of OPXV infections (see also Schmitt et al., 2014; Kramski et al., 2010). Mucker and colleagues successfully tested the susceptibility of CM to MPXV (Mucker et al., 2015). The CM were susceptible to MPXV but did not develop the classical pox-lesions (enanthema and exanthema) in contrast to the calpox virus where papulovesicular lesions were observed (Mätz-Rensing et al., 2012). Some of the main advantages of the here used calpox virus/marmoset model to other NHP models are their ability to work under BSL-2 conditions, the low infectious dose of calpox virus and the intranasal application route resembling the natural transmission route of human poxvirus infections. Furthermore, the disease in the calpox virus/marmoset model is comparable to human smallpox infections. Therefore, this model was used to test the efficacy of two attenuated smallpox vaccines MVA and MVTT with different vaccination strategies.

\subsection{Aims and Scope}

The aim of the study was to give insight into the early pathogenesis of CPVX infection in the calpox virus/marmoset model. The study aimed to determine the portal of viral entry, analyze the early dissemination as well as the pathological sequelae of infection in a time-course pathogenesis study.

The purpose of this study was also to analyze the immune response of the different vaccines and vaccination routes and the identification of protective immune correlates.

This work is therefore composed of two parts (i) a pathogenesis study and (ii) vaccine studies.

\subsubsection{Pathogenesis study}

Earlier studies conducted with calpox virus primarily focused on the final stage of disease with characteristic pox-like lesions detected in skin, mucous membranes, lymph nodes, liver and spleen (Mätz-Rensing et al., 2012). The main purpose of this study was the further characterization of calpox virus infection (1.2.4 Calpox virus) through the investigation of the early phase of the disease for clarification of the pathogenesis since several aspects are still unclear (e.g. mode of transmission, reason of death).

Therefore, a time-course pathogenesis study was conducted. Two different doses of calpox virus were used for intranasal inoculation of $15 \mathrm{CM}$. A group of three animals was infected with a high dose $\left(3.5 \times 10^{5} \mathrm{pfu}\right)$ of calpox virus and euthanized 3 dpi. Another group of twelve animals was infected with a 42-fold lower dose, with 10 median monkey infectious doses (MID 50$) 8.3 \times 10^{3}$ pfu of calpox virus. Each group consisted of three animals that were euthanized 5, 7, 10 and 12 days post inoculation. Serial necropsy was performed at predefined 
time points to collect different samples and detect different parameters in organs and blood using virological analyses (2.1.5 Pathogenesis study).

\subsubsection{Vaccination studies}

Two vaccination studies were conducted to evaluate the protective effect of differently attenuated vaccines after intranasal inoculation of a lethal dose of calpox virus in common CM (CM). Kramski and colleagues have already reported on the calpox virus/marmoset model as a suitable animal model for the evaluation of new vaccination strategies and antiviral therapies (Kramski et al., 2010).

Vaccination experiments have been conducted using VACV LE BN, MVA and MVTT.

The aim of this study was to analyze the cellular and humoral immune response of the different vaccines and vaccination routes and to identify immune correlates of protection retrospectively.

Studies at the German Primate Center (Deutsches Primatenzentrum GmbH, DPZ) have shown that intradermal VACV LE BN vaccination protected the four tested CM against a calpox virus challenge after a 10-week, but not after a 4-week waiting period (unpublished data; Dr. Stahl-Hennig and Dr. Mätz-Rensing, personal communication). VACV LE BN is the "gold standard" for vaccine efficacy in this animal model.

An early passage of MVA (MVA P568) was used in this study because the third generation smallpox vaccine Imvamune (also Imvanex or MVA BN) (Verardi et al., 2012) unexpectedly protected only two of four animals after a 4-week waiting period and did not induce protection after a 10-week waiting period in the calpox virus/marmoset model (Dr. Stahl-Hennig and Dr. Mätz-Rensing, personal communication) (1.9.2 Modified vaccinia virus Ankara (MVA)). Vaccination was done intramusculary (i.m.). While MVA provided solid protection in mouse and macaque models against MPXV infection, protection in the calpox virus/marmoset model is questionable. Here, CM were vaccinated intramuscularly with the early passage of MVA (MVA P568) and challenged with calpox virus after a four and 10-week waiting period.

Another attenuated vaccine used here was a MVTT, an attenuated variant of the vaccinia virus Tiantan that was used in China for vaccination against smallpox (Huang et al., 2009) (1.9.3 Modified vaccinia virus Tiantan (MVTT)). MVTT is replication competent in many mammalian cell lines but did not replicate in the mouse brain (Yu et al., 2010). Intranasal immunization with MVTT protected mice from VACV WR (Knitlova et al., 2014). It is more attenuated than VACV LE BN, but less than MVA which did not provide solid protection of the CM. 
In this study, CM were vaccinated with MVTT and challenged with calpox virus. Intranasally vaccinated macaques were challenged after a four and 10 -week waiting period while perorally vaccinated macaques were challenged after a 10 -week waiting period. 


\section{MATERIAL AND METHODS}

\subsection{Material}

The in this study used materials are listed in the Tables 2-1 - Table 2-6.

Table 2-1: General laboratory equipments and consumables.

\begin{tabular}{|c|c|}
\hline Name & Manufacturer \\
\hline$-20{ }^{\circ} \mathrm{C}$ freezer & Grundig \\
\hline$-20^{\circ} \mathrm{C}$ freezer, $4{ }^{\circ} \mathrm{C}$ fridge & Liebherr \\
\hline$-80{ }^{\circ} \mathrm{C}$ freezer ultralow u57085 & Labotect \\
\hline $4^{\circ} \mathrm{C}$ fridge eco energysaver & Privileg \\
\hline 5-Plex Rotor-Gene Q & Qiagen \\
\hline 96-well MaxiSorp Nunc-Immuno Plates & Thermo Scientific \\
\hline Agar dishes (100 mm) & Sarstedt \\
\hline Agilent DNA microarray scanner & Agilent Technologies \\
\hline Centrifugation tube $(15 \mathrm{ml}, 50 \mathrm{ml})$ & Greiner Bio-One \\
\hline Centrifuge 3 s-r & Heraeus \\
\hline Centrifuge $5415 \mathrm{R}$ & Eppendorf \\
\hline Centrifuge $5415 \mathrm{R}$ and 5424 & Eppendorf \\
\hline Centrifuge 5424 & Eppendorf \\
\hline Centrifuge sorvall discovery 90 with rotor tft 80.4 & ThermoScientific \\
\hline Cover slips & ThermoScientific \\
\hline Cryotubes $(1.8 \mathrm{ml}) \mathrm{NUNC}$ & ThermoScientific \\
\hline DNA-ExitusPlus & AppliChem \\
\hline Exsiccator, "Space Saver" F42025 & KNFLAB Neuberger \\
\hline FACS tubes & $\begin{array}{ll}\text { GmBH } \\
\text { Sarstedt }\end{array}$ \\
\hline fine scale $(0.1 \mathrm{mg}-200 \mathrm{~g})$ & Sartorius \\
\hline Flasks $\left(750 \mathrm{~mm}^{2}, 1750 \mathrm{~mm}^{2}\right)$ & Sarctedt \\
\hline Freezing device "mr. Frosty" & Nalcene \\
\hline Gel chamber & BIO-RAD \\
\hline Gel Documentation System & INTAS \\
\hline Glascontainers & Schott \\
\hline Harmony MIXER UZUSIO VTX-3000L & LLG. Lab Logistic Group \\
\hline Hera freeze $\left(-70^{\circ} \mathrm{C}\right)$ fridge & Heraeus \\
\hline Heraeus Thermo Scientific, Multifuge 3S-R & Thermo Scientific \\
\hline Hybridization System HybriWell HBW6L-2L & Grace Bio-Labs \\
\hline Ice mashine & Scotsman \\
\hline Incubation chamber, Rotilabo-Dying chamber Stain Tray & Carl Roth \\
\hline Incubator BBD6220 & Heraeus \\
\hline Incubator Heracell 240i & ThermoScientific \\
\hline Incubator series 5400 & NAPCO \\
\hline Inverted microscope, Axiovert 25 & Carl Zeics GmbH \\
\hline L-60 Ultraspeed Centrifuge & Beckmann Coulter \\
\hline LSR II (multi-laser flow cytometer) & BD Biosciences \\
\hline magnetic stirrer MR 2002 & \\
\hline Multi well plates (12-well, 351143; 96-well, 353072) & \\
\hline
\end{tabular}




\begin{tabular}{|c|c|}
\hline Multichannel micropipette & BD Falcon \\
\hline NanoDrop, ND-1000 Spectrophotometer & Eppendorf \\
\hline Neubauer counting chamber & NanoDrop Technologies \\
\hline Nitrogen tank & Carl Roth \\
\hline Nitrogen tank $-140{ }^{\circ} \mathrm{C}$ & Chronos Messer \\
\hline Non tissue culture treated plates (96-well) & Thermo Scientific \\
\hline Öko $-20^{\circ} \mathrm{C}$ & BD falcon \\
\hline Parafilm m & Privileg \\
\hline Pipette filter tips $(10 \mu 1,100 \mu 1,200 \mu 1,1000 \mu \mathrm{l})$ & Carl Roth \\
\hline Pipette tips rnase/dnase free $(10 \mu \mathrm{l}, 100 \mu \mathrm{l}, 200 \mu \mathrm{l}, 1000 \mu \mathrm{l})$ & Eppendorf \\
\hline Pipettes $(1-10 \mu 1,10-100 \mu 1,2-200 \mu 1,100-1000 \mu 1)$ & Sarstedt \\
\hline Polymax 1040 & Eppendorf \\
\hline PowerPac Basic power suppy & Heidolph \\
\hline Reaction tube $(0.2 \mathrm{ml}, 0.5 \mathrm{ml}, 1.5 \mathrm{ml}, 2 \mathrm{ml})$ & BIO-RAD \\
\hline Reaction tube $(1.5 \mathrm{ml}, 2 \mathrm{ml})$ & Sarstedt \\
\hline Rotilabo-Dying chamber and slide holder & Eppendorf \\
\hline Safe Lock Tubes $2.0 \mathrm{ml}$ & Carl Roth \\
\hline scale ED4202S-CW (0.01-4200 g) & Eppendorf \\
\hline Serological pipette $(2 \mathrm{ml}, 5 \mathrm{ml}, 10 \mathrm{ml}, 25 \mathrm{ml})$ & Sartorius \\
\hline SONOPLUS ultrasound-homogenizer & Sarstedt \\
\hline Sorvall Combi Plus Ultraspeed Centrifuge, DuPont & Bandelin \\
\hline Sorvall® RC-5B Refrigerated Superspeed Centrifuge; GS-3 & Thermo Scientific \\
\hline rotor & Thermo Scientific \\
\hline Sterile cell culture work bank & Thermo Scientific \\
\hline Tecan Sunrise photometer & ThermoScientific \\
\hline Tissue culture treated paltes (96-well) & Tecan Trading AG \\
\hline TissueLyser, Retsch & BD falcon \\
\hline TruCount tubes & Qiagen \\
\hline Vortex Genie 2 & BD Biosciences \\
\hline Water bath & Scientific Industries \\
\hline Water bath & GFL \\
\hline & Gebr. Rettberg \\
\hline
\end{tabular}

Table 2-2: Chemicals and Reagents.

\begin{tabular}{l|l}
\hline Reagent/ chemical & Source \\
\hline 3-Amino-9-EthylCarbazole & Sigma Aldrich \\
$3,3^{\prime}, 5,5^{\prime}$-Tetramethylbenzidine (TMB) Liquid Sub- & Sigma Aldrich \\
strate & Biozyme \\
Agrose & Roth \\
Albumin Fraction V & \\
anti-MVA rabbit immune serum & \\
anti-VACV serum & Invitrogen \\
Carboxyfluorescein succinimidyl ester & Roth \\
casein & SpheroTech \\
Compensation beads & Sigma Aldrich \\
Concanavalin-A & Sigma Aldrich \\
Crystal Violet & \\
\hline
\end{tabular}




\begin{tabular}{|c|c|}
\hline Dimethylformamide & Sigma Aldrich \\
\hline Dimethylsulfoxid (DMSO) & Fermentas \\
\hline dNTPs & Sigma Aldrich \\
\hline Ethanol & Merck \\
\hline Ethanol for spectroscopy & BD Biosciences \\
\hline FACS flow, FACS rinse, FACS safe & PAN Biotech \\
\hline Fetal calf serum & PAA laborato- \\
\hline Fetal calf serum & $\begin{array}{l}\text { ries } \\
\text { Roth }\end{array}$ \\
\hline Formaldehyde (37\%) & Sigma Aldrich \\
\hline glucose $\left(\mathrm{C}_{6} \mathrm{H}_{12} \mathrm{O}_{6}\right)$ & BioLegend \\
\hline Human TruStain FcX & Merck \\
\hline hydrogen chloride $(\mathrm{HCl})$ & \\
\hline $\begin{array}{l}\text { Hydrogen peroxide ( } 3 \% \text { ) } \\
\text { Isopropanol }\end{array}$ & Roth \\
\hline Loading dye $(6 x)$ & PAA laborato- \\
\hline Lymphocyte separation medium & ries \\
\hline Methanol & Roth \\
\hline Milk powder & Roth \\
\hline Nuclease free water & Merck \\
\hline Pancoll & PAN Biotech \\
\hline $\mathrm{PBS} / 0.5 \% \mathrm{BSA}$ & BioLegend \\
\hline Penicillin/streptomycin & PAN Biotech \\
\hline potassium chloride $(\mathrm{KCl})$ & Roth \\
\hline potassium dihydrogen phosphate $\left(\mathrm{KH}_{2} \mathrm{PO}_{4}\right)$ & Roth \\
\hline Primer & TIB Molbiol \\
\hline RCB Lysis/Fixation Solution (10x) & BioLegend \\
\hline RPMI- 1640 cell culture medium & PAN Biotech \\
\hline SecureSeal & Sigma Aldrich \\
\hline single-antibody-labeled compensation beads & SpheroTech \\
\hline Sodium acetate $(\mathrm{NaOAc})$ & Merck \\
\hline Sodium carbonate $\left(\mathrm{Na}_{2} \mathrm{CO}_{3}\right)$ & Merck \\
\hline sodium chloride $(\mathrm{NaCl})$ & Roth \\
\hline Sodium hydrogen carbonate $\left(\mathrm{NaHCO}_{3}\right)$ & Merck \\
\hline Sodium hydrogen phosphate $\left(\mathrm{Na}_{2} \mathrm{HPO}_{4} \cdot 12 \mathrm{H}_{2} \mathrm{O}\right)$ & Roth \\
\hline Streptavidin, V500 & BD Biosciences \\
\hline TaqMan probes & TIB Molbiol \\
\hline Trypsin, trypsin/EDTA $0.25 \%$ & PAN Biotech \\
\hline Tween 20 & Merck \\
\hline B-mercaptoethanol & Roth \\
\hline
\end{tabular}

Table 2-3: Reaction components and commercial kits.

\begin{tabular}{l|l}
\hline Kit/ component & Source \\
\hline Fixable Viability Kit, Brilliant Violet 570 & BioLegend \\
ImmoMix & Bioline \\
Plasmid DNA purification NucleoSpin Plasmid & Macherey- Nagel \\
QIAamp DNA Mini Kit (250) & Qiagen \\
TaqMan® Universal PCR Master Mix & Life Technologies \\
\hline
\end{tabular}


Table 2-4: List of Antibodies.

\begin{tabular}{|c|c|c|c|}
\hline Antigen & Clone & Fluorochrome & Manufacturer \\
\hline anti-CD45-Biotin & 6C9 & V500 & BD Biosciences \\
\hline anti-CD3 & SP $34-2$ & AF 700 & BD Biosciences \\
\hline anti-CD14 & M5E2 & PerCP-Cy5.5 & BD Biosciences \\
\hline anti-CD16 & $3 \mathrm{G} 8$ & APC-Cy7 & BD Biosciences \\
\hline anti-CD69 & L78 & PerCP-Сy5.5 & BD Biosciences \\
\hline anti-CD69 & FN50 & APC & BD Biosciences \\
\hline anti-CD4 & RPA-T4 & V450 & BD Biosciences \\
\hline anti-CD123 & 9F5 & $\mathrm{PE}$ & BD Biosciences \\
\hline anti-CD11b & ICRF44 & Pacific Blue & BD Biosciences \\
\hline anti-HLA-DR & L243 & APC-Cy7 & BD Biosciences \\
\hline anti-CD80 & L307.4 & V450 & BD Biosciences \\
\hline anti-CD20 & H299 (B1) & FITC & Beckman Coulter \\
\hline anti-CD159a & Z199.1 & APC & Beckman Coulter \\
\hline anti-CD45RA & $2 \mathrm{H} 4$ & ECD & Beckman Coulter \\
\hline anti-CD8 & HIT8a & $\mathrm{PE}$ & BioLegend \\
\hline anti-CD28 & L293 & PE-Cy7 & BioLegend \\
\hline anti-CD95 & DX2 & PE-Cy7 & BioLegend \\
\hline anti-CD95 & DX2 & APC & BioLegend \\
\hline anti-CD4 & L200 & PerCP-Cy5.5 & BioLegend \\
\hline anti-CD11c & 3.9 & PE-Cy7 & BioLegend \\
\hline anti-CD86 & IT2.2 & APC & BioLegend \\
\hline anti-human $\operatorname{IgGFc}$ & HP6017 & $\mathrm{PE}$ & BioLegend \\
\hline anti-CD27 & $\mathrm{O} 323$ & Brilliant Violet 650 & BioLegend \\
\hline 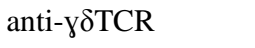 & 5A6.E9 & FITC & Caltag Laboratories \\
\hline anti-CD8a & DK25 & $\mathrm{PE}$ & Dako \\
\hline
\end{tabular}

Table 2-5: List of secondary Antibodies.

\begin{tabular}{l|l}
\hline Antigen & Manufacturer \\
\hline Alexa Fluor 647 peroxidase-conjugated AffiniPure $\mathrm{F}\left(\mathrm{ab}^{\prime}\right)_{2}$, & \\
$\left(\mathrm{H}+\mathrm{L}^{1}\right)$-conjugated goat anti-human IgG antibody & \\
$\mathrm{Cy5}$-conjugated AffiniPure $\mathrm{F}\left(\mathrm{ab}^{\prime}\right)_{2},\left(\mathrm{H}+\mathrm{L}^{1}\right)_{\text {-conjugated goat }}$ & \\
anti-mouse IgG antibody & Jackson ImmunoResearch Laboratories \\
Alexa Fluor 647-conjugated AffiniPure $\mathrm{F}\left(\mathrm{ab}^{\prime}\right)_{2}$ fragment goat & \\
anti-human IgG $\left(\mathrm{H}+\mathrm{L}^{1}\right)$ & \\
Cy3-conjugated IgG Fraction Monoclonal Mouse Anti-Biotin & \\
\hline
\end{tabular}

${ }^{1}$ detects heavy and light side chains

Table 2-6: List of buffers and medium.

\begin{tabular}{l|l}
\hline Reagent/ chemical & Source/ ingredients \\
\hline FOXP3 Fix/Perm Buffer Set & BioLegend \\
RBC Lysis/Fixation, 10x stock, diluted & BioLegend \\
with $\mathrm{ddH}_{2} \mathrm{O}$ to 1x Solution & \\
PBS/BSA & PBS/0.5\% BSA \\
\hline
\end{tabular}




\begin{tabular}{|c|c|}
\hline $\begin{array}{l}\text { FA-solution, } 0.37 \% \text { formaldehyde (FA)- } \\
\text { solution }\end{array}$ & diluted in PBS \\
\hline Blocking buffer & $2 \%(\mathrm{w} / \mathrm{v})$ milk powder $+10 \%(\mathrm{v} / \mathrm{v}) \mathrm{FCS}$ in PBS \\
\hline Coating buffer & $\begin{array}{l}\text { sodium carbonate }\left(\mathrm{Na}_{2} \mathrm{CO}_{3}\right) \text { buffer, } 0.03 \mathrm{M}(\mathrm{w} / \mathrm{v}), \mathrm{pH} 11 \text {, diluted } 1: 2 \text { in sodium } \\
\text { hydrogen carbonate }\left(\mathrm{NaHCO}_{3}\right) \text { buffer, } 0.07 \mathrm{M}(\mathrm{w} / \mathrm{v}), \mathrm{pH} 8 \\
1 \%(\mathrm{w} / \mathrm{v}) \text { sodium chloride }(\mathrm{NaCl}), 0.02 \%(\mathrm{w} / \mathrm{v}) \text { potassium chloride }(\mathrm{KCl}) \text {, } \\
0.289 \%(\mathrm{w} / \mathrm{v}) \text { sodium hydrogen phosphate }\left(\mathrm{Na}_{2} \mathrm{HPO}_{4} \cdot 12 \mathrm{H}_{2} \mathrm{O}\right), 0.02 \%(\mathrm{w} / \mathrm{v})\end{array}$ \\
\hline Phosphate-buffered saline (PBS) & $\begin{array}{l}14 \mathrm{mM} \text { potassium dihydrogen phosphate }\left(\mathrm{KH}_{2} \mathrm{PO}_{4} \cdot 12 \mathrm{H}_{2} \mathrm{O}\right), 1.37 \mathrm{mM} \text { sodium } \\
\text { chloride }(\mathrm{NaCl}), 27 \mathrm{mM} \text { potassium chloride }(\mathrm{KCl}), 43 \mathrm{mM} \text { sodium hydrogen } \\
\text { phosphate }\left(\mathrm{Na}_{2} \mathrm{HPO}_{4}\right)\end{array}$ \\
\hline $\begin{array}{l}\text { Dulbecco's Modified Eagle Medium } \\
\text { (DMEM) with } 4.5 \mathrm{~g} / \mathrm{L} \text { Glucose }\end{array}$ & PAN-Biotech \\
\hline tissue DMEM medium & $\begin{array}{l}\text { DMEM, } 2 \% \text { fetal calf serum (FCS) and a mixture of antibiotics ( } 1 \% \text { penicillin- } \\
\text { streptomycin; } 50 \mu \mathrm{g} / \mathrm{ml} \text { neomycin; } 100 \mu \mathrm{l} / \mathrm{ml} \text { nystatin; } 1 \mu \mathrm{g} / \mathrm{ml} \text { amphotericin and } \\
1 \% \text { gentamycin) }\end{array}$ \\
\hline $\begin{array}{l}\text { TMB-Substrate, } 3,3^{\prime}, 5,5^{\prime} \text { Tetra- } \\
\text { methylbenzidine (TMB) Liquid Substrate } \\
\text { solution, System for ELISA, T0440 }\end{array}$ & Sigma Aldrich \\
\hline $1 \mathrm{mM}$ TRIS buffer & 1 mM TRIS, pH 9.0 \\
\hline Stop solution & Hydrogen chloride, $1 \mathrm{M}$ \\
\hline $\mathrm{Na}_{2} \mathrm{CO}_{3}$ buffer & $\begin{array}{l}0.03 \mathrm{M}(\mathrm{w} / \mathrm{v}), \mathrm{pH} 11 \text {, diluted } 1: 2 \text { in sodium hydrogen carbonate } \mathrm{NaHCO}_{3} \text { buffer } \\
(0.07 \mathrm{M}(\mathrm{w} / \mathrm{v}), \mathrm{pH} 8)\end{array}$ \\
\hline Blocking buffer & $2 \%(\mathrm{w} / \mathrm{v})$ milk powder $+10 \%(\mathrm{v} / \mathrm{v}) \mathrm{FCS}$ in PBS \\
\hline
\end{tabular}

\subsection{Animal experiments}

\subsubsection{Ethical statement}

All animal experiments were performed at the German Primate Centre Göttingen (Deutsches Primatenzentrum GmbH, DPZ) and followed the laws of the German Animal Welfare Act. It complies with the European Union guidelines (EU directive 2010/63/EU) on the use of nonhuman primates for biomedical research.

Animal experiments were supervised by governmental veterinary authorities. The experiments were performed under licence number 33.9-42502-04-12/0745 issued by the LAVES (Niedersächsisches Landesamt für Verbraucherschutz und Lebensmittelsicherheit).

\subsubsection{Non-human primates and animal husbandry}

All animals used for the present studies were bred and housed at the DPZ under standard conditions. In total, 45 common CM (CM, Callithrix jacchus) were used for the present studies. 
19 animals entered the pathogenesis study and 26 animals were used for the vaccination studies (Table 2-7 and 2-8).

Prior to the experiments, CM were kept in the animal unit at Biosafety Level 2 (BSL-2) at the DPZ for acclimatisation purposes. They were housed in individual cages $(130 \times 58 \times 80 \mathrm{~cm})$, at room temperature $\left(25^{\circ} \mathrm{C} \pm 1{ }^{\circ} \mathrm{C}\right)$. At any time, they had the opportunity to get in touch with each other. Balanced diet consisted of food pellets for primates supplemented with fresh fruits and mash. Fresh water was provided ad libitum. Naïve animals served as negative controls for the detection of calpox antigen by flow cytometry (3.2.2 Detection of calpox virus antigen in various immune cells by flow cytometry).

Table 2-7: Specification of common CM (Callithrix jacchus) used for the pathogenesis study.

\begin{tabular}{|c|c|c|c|c|}
\hline Group & Animal ID & Sex & $\begin{array}{l}\text { Age } \\
\text { (years) }\end{array}$ & $\begin{array}{l}\text { Euthanasia } \\
\text { (dpi) }\end{array}$ \\
\hline \multirow[t]{3}{*}{ I } & 14325 & $\mathrm{~m}$ & 6 & $3^{1}$ \\
\hline & 14484 & $\mathrm{~m}$ & 5 & $3^{1}$ \\
\hline & 14991 & $\mathrm{~m}$ & 6 & $3^{1}$ \\
\hline \multirow[t]{3}{*}{ II } & 14973 & $\mathrm{f}$ & 3 & $5^{2}$ \\
\hline & 14592 & $\mathrm{f}$ & 4 & $5^{2}$ \\
\hline & 14647 & $\mathrm{~m}$ & 4 & $5^{2}$ \\
\hline \multirow[t]{3}{*}{ III } & 14483 & $\mathrm{f}$ & 4 & $7^{2}$ \\
\hline & 14966 & $\mathrm{~m}$ & 3 & $7^{2}$ \\
\hline & 14917 & $\mathrm{f}$ & 3 & $7^{2}$ \\
\hline \multirow[t]{3}{*}{ IV } & 15149 & $\mathrm{f}$ & 2 & $10^{2}$ \\
\hline & 15121 & $\mathrm{f}$ & 3 & $10^{2}$ \\
\hline & 14306 & $\mathrm{f}$ & 4 & $10^{2}$ \\
\hline \multirow[t]{3}{*}{$\mathrm{V}$} & 14702 & $\mathrm{~m}$ & 5 & $12^{2}$ \\
\hline & 15122 & $\mathrm{~m}$ & 5 & $12^{2}$ \\
\hline & 15095 & $\mathrm{~m}$ & 5 & $12^{2,3}$ \\
\hline \multirow[t]{4}{*}{ control } & 15995 & $\mathrm{f}$ & 5 & -4 \\
\hline & 15968 & $\mathrm{f}$ & 5 & -4 \\
\hline & 16000 & $\mathrm{~m}$ & 5 & -4 \\
\hline & 15970 & $\mathrm{~m}$ & 5 & -4 \\
\hline
\end{tabular}

${ }^{\mathbf{1}}$ Animals were infected with $3.5 \times 10^{5}$ pfu calpox virus.

${ }^{2}$ Animals were infected with $8.3 \times 10^{3}$ pfu calpox virus.

${ }^{3}$ Animal was at the final stage of disease.

${ }^{4}$ Naïve animals, not infected.

dpi, days post infection; f., female; m., male.

Table 2-8: Specification of common CM (Callithrix jacchus) used for the vaccination studies.

\begin{tabular}{|c|c|c|c|c|c|c|}
\hline Study & $\begin{array}{l}\text { Vaccination } \\
\text { group }\end{array}$ & Vaccine & Animal ID & Sex & Age & $\begin{array}{ll}\text { Waiting period } & \text { timepoint } \\
\text { between second } & \text { of death } \\
\text { immunisation } & \text { (dpi) } \\
\text { and challenge }^{1} & \end{array}$ \\
\hline
\end{tabular}




\begin{tabular}{|c|c|c|c|c|c|c|c|}
\hline \multirow[t]{12}{*}{ Study I } & \multirow[t]{4}{*}{ I } & \multirow[t]{4}{*}{ MVA i.m. } & 14144 & $f$ & 5 & \multirow[t]{4}{*}{4 weeks } & $24 *$ \\
\hline & & & 14403 & $\mathrm{f}$ & 5 & & $21 *$ \\
\hline & & & 14482 & $\mathrm{~m}$ & 5 & & $23 *$ \\
\hline & & & 14707 & $\mathrm{f}$ & 4 & & $18 * * *$ \\
\hline & \multirow[t]{2}{*}{ control } & \multirow[t]{2}{*}{ PBS i.m. } & 14406 & $\mathrm{~m}$ & 5 & \multirow[t]{2}{*}{4 weeks } & $13^{*}$ \\
\hline & & & 14646 & $\mathrm{~m}$ & 5 & & $14 *$ \\
\hline & \multirow[t]{4}{*}{ II } & \multirow[t]{4}{*}{ MVTT i.n. } & 14327 & $\mathrm{f}$ & 5 & \multirow[t]{4}{*}{4 weeks } & $84 * *$ \\
\hline & & & 14414 & $\mathrm{f}$ & 5 & & $90 * *$ \\
\hline & & & 14575 & $\mathrm{~m}$ & 5 & & $90 * *$ \\
\hline & & & 15007 & $\mathrm{f}$ & 4 & & $19^{*}$ \\
\hline & \multirow[t]{2}{*}{ control } & \multirow[t]{2}{*}{ PBS i.n. } & 15006 & f & 4 & \multirow[t]{2}{*}{4 weeks } & $16 * * *$ \\
\hline & & & 14415 & $\mathrm{f}$ & 5 & & $12 * * *$ \\
\hline \multirow[t]{14}{*}{ Study II } & \multirow[t]{4}{*}{ III } & \multirow[t]{4}{*}{ MVA i.m. } & 14233 & $\mathrm{~m}$ & 6 & \multirow[t]{4}{*}{10 weeks } & $17 *$ \\
\hline & & & 14309 & $\mathrm{f}$ & 6 & & $99 * *$ \\
\hline & & & 14248 & $\mathrm{~m}$ & 6 & & $21^{*}$ \\
\hline & & & 14372 & $\mathrm{~m}$ & 6 & & $21 *$ \\
\hline & \multirow[t]{4}{*}{ IV } & \multirow[t]{4}{*}{ MVTT i.n. } & 14169 & $\mathrm{~m}$ & 6 & \multirow[t]{4}{*}{10 weeks } & $17 *$ \\
\hline & & & 14603 & $\mathrm{f}$ & 6 & & $16^{*}$ \\
\hline & & & 14409 & $\mathrm{~m}$ & 6 & & $99 * *$ \\
\hline & & & 14469 & $\mathrm{f}$ & 6 & & $101 * *$ \\
\hline & control & PBS i.n. & 14365 & $\mathrm{f}$ & 5 & 10 weeks & $12 * * *$ \\
\hline & \multirow[t]{4}{*}{$\mathrm{V}$} & \multirow[t]{4}{*}{ MVTT p.o. } & 15098 & $\mathrm{~m}$ & 5 & \multirow[t]{4}{*}{10 weeks } & $98 * *$ \\
\hline & & & 14478 & $\mathrm{f}$ & 6 & & $101 * *$ \\
\hline & & & 14334 & $\mathrm{~m}$ & 6 & & $98 * *$ \\
\hline & & & 15100 & $\mathrm{~m}$ & 5 & & $17 *$ \\
\hline & control & PBS p.o. & 14974 & $\mathrm{~m}$ & 5 & 10 weeks & $14 *$ \\
\hline
\end{tabular}

${ }^{\mathbf{1}}$ The boost immunisation (control groups were mock-immunized with PBS) followed four weeks after the first vaccination in study I and ten weeks after first vaccination in study II. *Euthanasia because termination score was reached. **euthanasia at the end of the experiment. ***sudden death. dpi, days post infection; f., female; i.m., intramuscular; i.n., intranasal; MVA, modified vaccinia virus Ankara ; MVTT, modified vaccinia virus Tiantan; m., male; PBS, phosphate-buffered saline; p.o., peroral.

\subsubsection{Necropsy}

All CM were adspected daily and checked for clinical symptoms. For the pathogenesis study animals were euthanised at predefined time points (2.1.6 Pathogenesis study). Serial necropsy was then performed at 3, 5, 7, 10 and 12 dpi.

In the vaccination studies, animals were euthanized when the level of the predefined humane endpoint criteria was reached.

Animals were anaesthetized with Göttinger Mixture II (GM II) (see Appendix: Table A.2-1)

before intraperitoneal euthanasation with $100 \mathrm{mg} / \mathrm{kg}$ pentobarbital-sodium (Narcoren, Merial

GmbH, Hallbergmoos, Germany). 


\subsubsection{Sampling}

\section{Tissue}

Tissue samples of 29 organs (adrenal gland, axillary lymph node (LN), urinary bladder, bone marrow, buccal musoca, central nervous system (CNS), colon, esophagus, eyes, heart, inguinal LN, kidney, liver, lung, mesenteric LN, muscle, nasal mucosa, ovar/epididymis, parotid gland, skin, small intestine, spleen, stomach, submandibular LN, tongue, tonsil, trachea, uterus/prostate, vagina/testicle) per animal were collected and stored $-80{ }^{\circ} \mathrm{C}$.

\section{Blood samples}

For taking blood animals were anaesthetized with 20-30 mg ketamine/kg body weight intramuscularly (i.m.). Syringes used for blood drawing contained EDTA to avoid clotting. Blood samples were collected at 0,3, 5, 7, 10 and 12 dpi for the pathogenesis study. Samples were taken for the vaccination study I at $0,5,7,10,12,14,17,19,21,28,42,56,72$ dpi and for the vaccination study II at $0,5,7,10,12,14,17,21,25,29,35,42,49$ dpi.

\section{Plasma samples}

For vaccination study I and II plasma samples were obtained prior to vaccination and at 0 and $14 \mathrm{dpi}$ as well as at the latest available time points for serological assays.

Plasma was either prepared from whole blood by centrifugation for $15 \mathrm{~min}$ at $3000 \mathrm{rpm}$ or by centrifugation on a ficoll gradient for $25 \mathrm{~min}$ at $800 \mathrm{~g}$.

\subsubsection{Design of the pathogenesis study}

For the pathogenesis study, in total 15 animals were infected intranasally with $100 \mu 1$ of different doses of calpox virus suspension (Table 2-7). Initially, twelve animals were infected, three each group, with 10 median monkey infectious doses $\left(\mathrm{MID}_{50}\right)$ corresponding to $8.3 \mathrm{x}$ $10^{3}$ plaque forming units ( $\mathrm{pfu}$ ) of calpox virus according to Kramski et al. (2010), and three each were sacrificed at days 5, 7, 10 and 12 post inoculation (Figure 2-1). Because most samples from 5-dpi-animals were virus negative, another group of three animals was infected with a 42 -fold higher dose $\left(3.5 \times 10^{5} \mathrm{pfu}\right)$ of calpox virus and euthanised at $3 \mathrm{dpi}$. All animals were infected by pipetting $50 \mu 1$ of the calpox virus suspension into each nostril. 


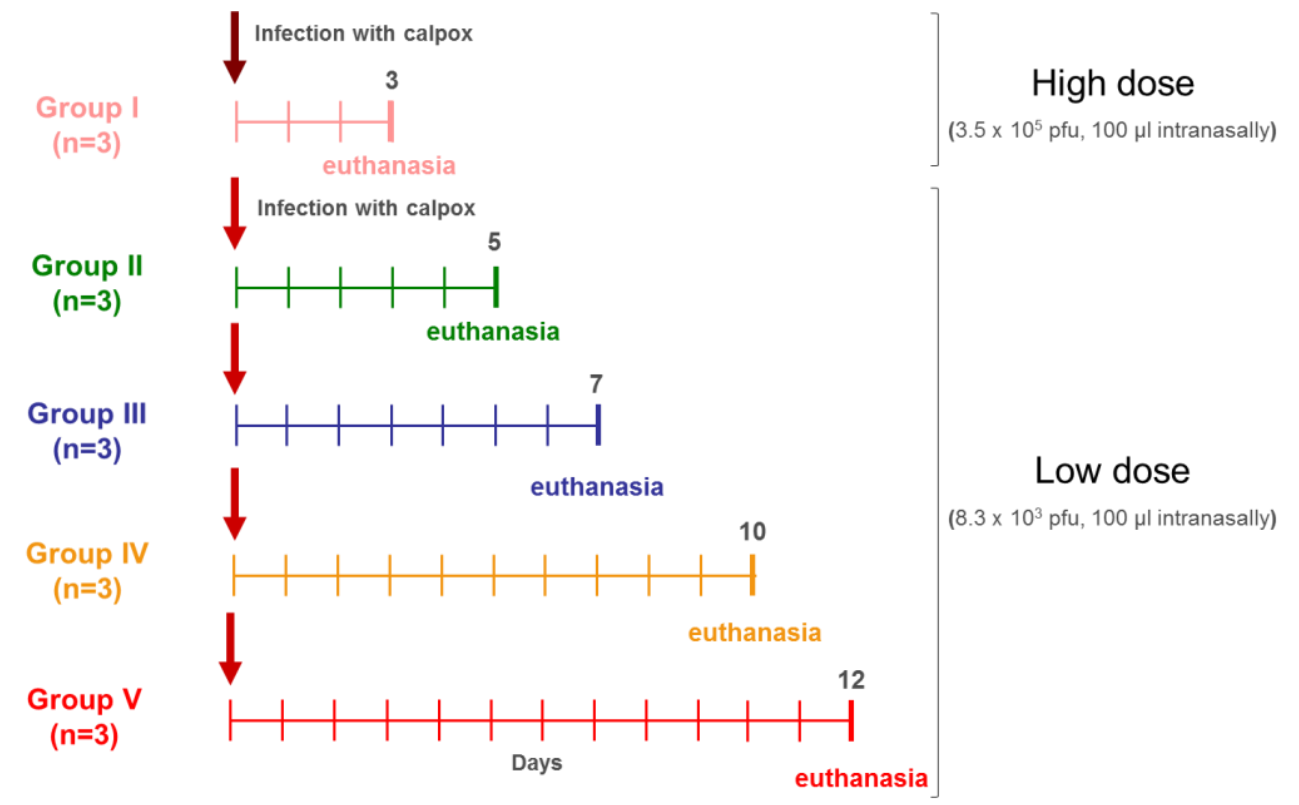

Figure 2-1: Design of the pathogenesis study. Three animals (group I, high dose) were infected intranasally with $3.5 \times 10^{5}$ pfu of calpox virus and were sacrificed 3 days post infection. Each three CM of group II-V (low dose), were inoculated intranasally with 10 median monkey infectious doses ( $\mathrm{MID}_{50}$ ) corresponding to $8.3 \times 10^{3}$ plaque forming units (pfu) of calpox virus and euthanised at days 5, 7, 10 and 12 post inoculation (low dose).

\section{Analysis plan of the pathogenesis study}

In total 29 organs (2.1.5 Sampling) per NHP were collected and tissue homogenates were prepared for virus titration and quantification of viral DNA by real-time polymerase chain reaction (PCR). In addition, $1 \mathrm{ml}$ blood was collected from each animal for viral DNA quantification at the day of necropsy and only day 3 and day 12 animals for virus titration. Additonally, in the day-3 and day-12 groups the presence of calpox virus antigen was determined by flowcytometric analysis of purified PBMCs and buffy coat.

\subsubsection{Vaccination studies}

Two vaccination studies were performed in the calpox virus/marmoset model to test the efficacy of different live-attenuated vaccinia viruses (VACV), i.e. modified VACV Ankara (MVA) (kindly provided by Prof. Czerny, Microbiology and Animal Hygiene, Georg-August University Göttingen) and modified VACV Tiantan (MVTT) (kindly provided by Prof. Chen, AIDS Institute, Hong Kong University, China).

\section{Vaccination study I: Immunization and challenge}

Twelve animals were used for the vaccine study I (Figure 2-2) and distributed to three groups with four $\mathrm{CM}$ each. One group was immunized intranasally with $5 \times 10^{7}$ plaque forming units (pfu) of MVTT. 50 $\mu 1$ of the virus suspension was administered into each nostril. Another 
group received MVA passage 568 intramuscularly at the same dose like that used for immunization with MVTT. The applied volume was $1 \mathrm{ml}$, and $500 \mu \mathrm{l}$ were injected into each hamstring muscle. Four weeks after the final immunization (four-week waiting period) all vaccinees together with four mock-treated controls were exposed intranasally to $8.3 \times 10^{3}$ pfu calpox virus as described above (2.2.5 Design of pathogenesis study). Animals of the control group were mock immunized with PBS (Table 2-8).

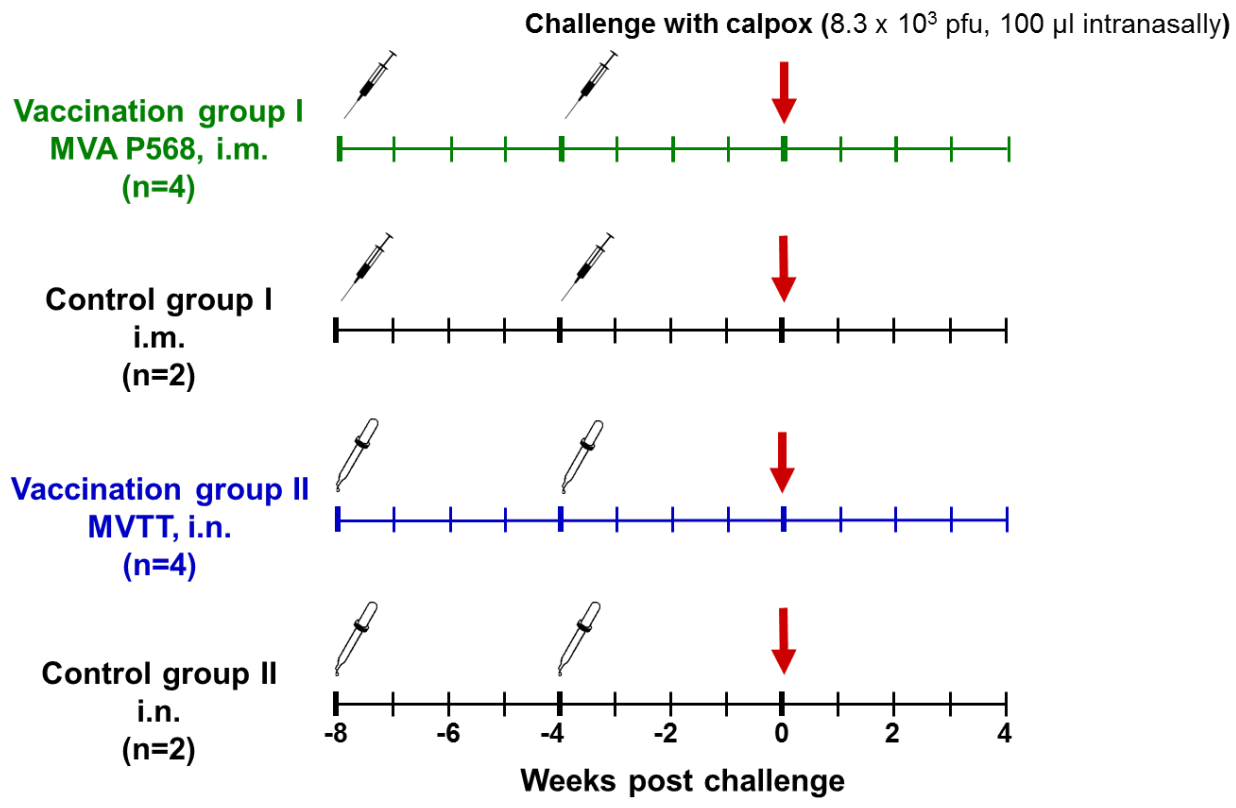

Figure 2-2: Scheme of vaccination study I. Four animals were immunized intramuscularly (i.m.) with $5 \times 10^{7}$ pfu of Modified Vaccinia virus Ankara (MVA) passage (P) 568 (vaccination group I). Vaccination group II was immunized with $5 \times 10^{7}$ pfu of modified vaccinia virus Tiantan strain (MVTT) intranasally (i.n.). The respective control groups were mock immunized. All animals were challenged i.n. with calpox virus four weeks after the second immunisation.

\section{Vaccination study II}

In the follow-up study (vaccination study II) fourteen CM were used (Figure 2-3). Four animals each were allocated to three vaccine groups, two mock-immunized animals served as controls. Two groups were identically immunized as described for vaccination study I. The third group received the same dose of MVTT identically as outlined above, but by the peroral route. For immunization with MVTT the two mucosal application sites were chosen because mucosal vaccination with the parental VTT in mice (i.n. or oral) induced higher and more sustained levels of neutralizing antibodies then the i.m. and subcutaneous vaccination routes ( $\mathrm{Lu}$ et al., 2011). In contrast to vaccination study I the time span between the booster immunisation and challenge virus exposure was prolonged to a 10 -week waiting period to potentially 
increase the protection of the CM. The challenge was performed as described (2.2.5 Design of pathogenesis study).

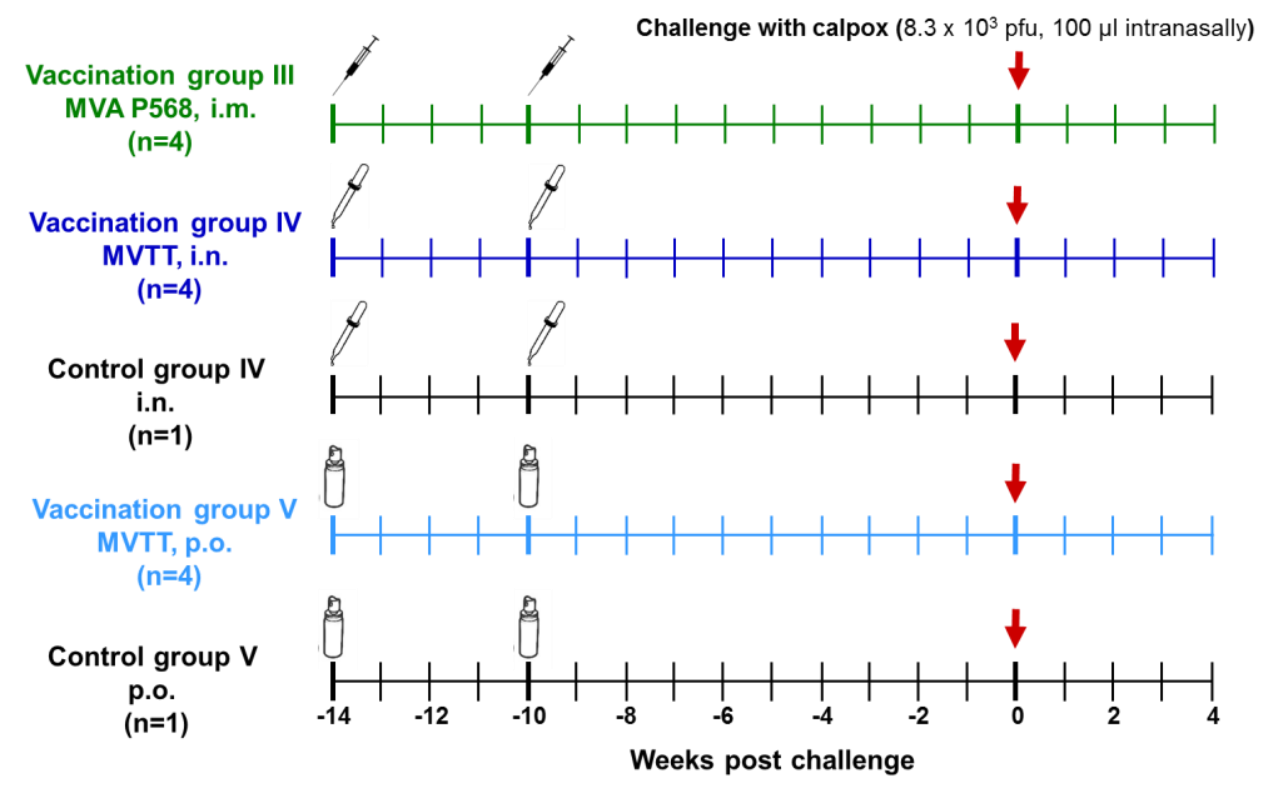

Figure 2-3: Scheme of vaccination study II. The study design was modified from vaccination study I. Animals of Vaccination group III were immunized intramuscularly (i.m.) with $5 \times 10^{7} \mathrm{pfu}$ of modified vaccinia virus Ankara (MVA) passage (P) 568. Vaccination group IV was immunized with $5 \times 10^{7} \mathrm{pfu}$ of modified vaccinia virus Tiantan strain (MVTT) intranasally (i.n.). Peroral (p.o.) immunisation with $5 \times 10^{7}$ pfu of MVTT was included as vaccination group V. There was a 10 -week waiting period between last vaccination and challenge with calpox virus. The control animals were mock immunized. i.m., intramuscularly; i.n., intranasally; MVA, Modified Vaccinia virus Ankara; P, passage.

\section{Analysis plan of the Vaccination studies}

Part of the results of vaccination study I were already compiled before the beginning of this thesis (detection and quantification of viral DNA copies in blood by real time-PCR). Therefore, this study predominantly focused on the analysis of vaccination study II. The humoral response in plasma was analysed by ELISA, neutralisation assay, and antibody fine mapping by peptide microarray. In order to analyse subsets of innate and adaptive immune cells in whole blood, polychromatic flow cytometry was applied. The cellular response was analysed with the T cell proliferation assay using carboxyfluorescein succinimidyl ester (CFSE) for cell labelling. Calpox virus in blood was determined by virus isolation and real-time PCR.

\subsection{Cell culture}

Vero E6 cells are susceptible to calpox virus infection and were therefore used for analyses in these presented studies. According to the recommendations of the European Collection of Cell Cultures, Vero E6 cells were cultivated with Dulbecco's Modified Eagle Medium (DMEM) with $4.5 \mathrm{~g} / \mathrm{L}$ Glucose (PAN-Biotech GmbH, Aidenbach, Germany), $10 \%$ FCS and 
$1 \%$ Penicillin-Streptomycin in culture flasks (Sarstedt, Nümbrecht, Germany). When the cells reached $90 \%$ confluence, they were washed once with phosphate-buffered saline (PBS) and incubated with trypsin/EDTA (PAN-Biotech GmbH, Aidenbach, Germany) at $37^{\circ} \mathrm{C}$. After cells were properly detached from the surface of the culture flask which required approximately 5-7 min, they were washed in Vero E6 cell culture medium once. Thereafter, they were split 1:10 and seeded into new culture flasks.

\subsection{Viruses}

Calpox virus Calpox virus was isolated from the skin of an infected Callithrix jacchus, 2002, Robert Koch Insitute, Berlin, Germany, kindly provided by Dr. Ellerbrok, Robert Koch Institut, Germany

VACV LE Vaccinia virus Lister Elstree, kindly provided by Prof. Czerny, Division of Microbiology and Animal Hygiene, Georg-August University of Goettingen, Germany

MVTT Modified vaccinia virus Tiantan, working stock was kindly provided by Prof. Chen, AIDS Institute and Department of Microbiology, University of Hong Kong, China

MVA, passage 568 Modified vaccinia virus Ankara, kindly provided by Prof. Czerny, Division of Microbiology and Animal Hygiene, Georg-August University of Goettingen, Germany

\subsection{Isolation of peripheral blood mononuclear cells (PBMCs) and buffy coat from whole blood}

Whole blood was diluted 1:2 with PBS and added onto Pancoll (PAN-Biotech GmbH, Aidenbach, Germany). A centrifugation step followed at $800 \mathrm{x}$ g for $25 \mathrm{~min}$ at room temperature. PBMCs banded at the interphase between Pancoll and plasma. Plasma was removed and stored at $-80{ }^{\circ} \mathrm{C}$ for later analysis. Next, the PBMCs were removed and washed twice with PBS which included centrifugation at 135 relative centrifugal force (rcf) or $\mathrm{xg}$ (times gravity) for $10 \mathrm{~min}$ at room temperature in a total volume of $10 \mathrm{ml}$. Finally, the cell pellet was resuspended in $1 \mathrm{ml}$ PBMC cell culture medium (RPMI-1640, PAN-Biotech GmbH, Aidenbach, Germany, $+10 \% \mathrm{FCS},+1 \% \mathrm{P} / \mathrm{S})$ and cell numbers were counted with a hemocytometer $(2.3$ Cell culture). Purified cells were either immediately processed for further analyzes or shortly stored in the refrigerator. 
Buffy coat was isolated from $600 \mu \mathrm{l}$ of whole blood which was centrifuged for $15 \mathrm{~min}$ at 564 $\mathrm{x}$ g. EDTA plasma was separated and stored at $-20{ }^{\circ} \mathrm{C}$ for serological analyses. The phase under the plasma contained the buffy coat and was visible as a white layer. The buffy coat was aspirated and washed in $1 \mathrm{ml}$ PBMC cell culture medium, centrifuged for $5 \mathrm{~min}$ at $1200 \mathrm{rpm}$ and the supernatant discarded. The pellet was resuspended in $120 \mu 1$ PBS and $50 \mu 1$ were used for flow cytometric analysis.

\subsection{Extraction of nucleic acid}

\subsubsection{DNA extraction from blood samples}

Calpox virus DNA was extracted from whole blood. To this end, $100 \mu 1$ of whole blood was diluted 1:2 with PBS. For DNA extraction the manufacturer's protocol of the QIAamp® DNA Mini Kit was followed except that the DNA was finally eluted in $100 \mu \mathrm{AE}$ buffer provided from the kit. Eluates containing the DNA were stored at $-20{ }^{\circ} \mathrm{C}$.

\subsubsection{DNA extraction from tissue samples}

Calpox virus DNA was extracted from 29 tissue samples per animal (2.2.5 Design of the pathogenesis study). After defining the weight of each tissue sample, PBS and one $5 \mathrm{~mm}$ diameter steel beads were added. The TissueLyser II (Qiagen) was then used for homogenisation. The homogenisation times varied among tissues (20 sec $-3 \mathrm{~min}$ ). The supernatant was taken out gently after a centrifugation step (125 x g for $10 \mathrm{~min}$ ). Aliquots of $100 \mu \mathrm{l}$ served as the base for DNA extraction and for plaque assay. Manufacturer's protocol of the QIAamp® DNA Mini Kit (Qiagen) was followed for DNA extraction from tissues. DNA was eluated in $100 \mu \mathrm{AE}$ buffer and stored at $-20^{\circ} \mathrm{C}$.

\subsection{Polychromatic flow cytometry}

Cells were stained with antibodies conjugated with fluorescent dyes and acquired using a LSR II (BD) equipped with three lasers (violet, blue and red). Compensation was calculated using appropriate single-antibody-labeled compensation beads (SpheroTech) to correct the overlapping of emission spectra. The calculation was done by FACS DIVA software 6.1.3. Analysis was done with FlowJo 9.6 (Treestar, Ashland, Oregon). 


\subsubsection{Preparation of compensation controls}

Compensations were calculated with compensation beads (SpheroTech, Lake Forest, Illinois) and the respective antibodies and dyes. Compensation beads were resuspended in PBS and 20 $\mu 1$ of this solution were added to FACS tubes. $1 \mu 1$ of each antibody listed below was added to the respective FACS tube and the suspension mixed Beads were incubated at room temperature for $30 \mathrm{~min}$ in the dark. Next, $500 \mu \mathrm{l}$ of RBC Lysis/Fixation solution (1x) were added. After vortexing and incubation for $15 \mathrm{~min}$ in the dark at room temperature, the mixture was centrifuged for $5 \mathrm{~min}$ at $1200 \mathrm{rpm}$ and the supernatant discarded. $250 \mu 1 \mathrm{PBS} / \mathrm{BSA}$ were added, the mixture was vortexed and centrifuged. The supernatant was discarded and the singlestained compensation beads were resuspended in $50 \mu \mathrm{l}$ PBS/BSA, acquired and the compensation was calculated using the FACS DIVA software 6.1.3.

\subsubsection{Polychromatic flow cytometric staining for calpox antigen in PBMCs}

PBMCs were stained and analysed by polychromatic flow cytometry in order to identify the type of immune cells infected with calpox virus. Blood from all animals sacrificed at the predefined time points day 3 and 12 post infections were available for this kind of analysis (2.2.5 Design of the pathogenesis study). First, $5 \times 10^{5}$ PBMCs purified from whole blood were stained for a number of cell surface markers. The staining cocktail comprised the following antibody conjugates: anti-human IgG Fc-PE, anti-CD45-Biotin, anti-CD3-AF700, anti-CD14PerCP-Cy5.5, anti-CD16- APC-Cy7, anti-CD20-FITC, and anti-CD159a-APC, and a streptavidin-V500 conjugate. All antibodies and the streptavidin were used at pretitrated concentrations. As a very first step, anti-CD45-Biotin was preincubated with Streptavidin-V500 for $15 \mathrm{~min}$ (1:1000). $50 \mu \mathrm{l}$ of the CD45-Biotin/Streptavidin mixture per test was added to $8 \mu \mathrm{l}$ of the mixture of the antibody cocktail. This mixture ( $58 \mu 1 /$ test $)$ was added to FACS tubes. $5 \mathrm{x}$ $10^{5}$ PBMCs in $50 \mu 1$ PBS/BSA were added and incubated with the antibodies for $30 \mathrm{~min}$ in the dark at room temperature (RT). Thereafter, one ml FOXP3 FIX/Perm Buffer (1x) was added, the mixture vortexed and incubated for $20 \mathrm{~min}$ in the dark at RT. Following centrifugation for $5 \mathrm{~min}$ at $1200 \mathrm{rpm}$, the supernatant was discarded, and cells were resuspended in $250 \mu \mathrm{PBS} / \mathrm{BSA}$ and stored at $4{ }^{\circ} \mathrm{C}$ over night. The next day PBMCs were pelleted for $5 \mathrm{~min}$ at $1200 \mathrm{rpm}$, the supernatant discarded, and $1 \mathrm{ml} \mathrm{FOXP3} \mathrm{Perm} \mathrm{Buffer} \mathrm{added.} \mathrm{The} \mathrm{mixture}$ was then vortexed and incubated for $20 \mathrm{~min}$ in the dark at RT followed by cell pelleting for 5 min at $1200 \mathrm{rpm}$. After that, the supernatant was discarded, $250 \mu 1$ Perm Buffer added, and the mixture vortexed. The samples were centrifuged for $5 \mathrm{~min}$ at $1200 \mathrm{rpm}$ and the supernatant was discarded again. $500 \mu \mathrm{l}$ Perm Buffer were added, the mixture vortexed and incubated 
for $15 \mathrm{~min}$ in the dark at RT. The samples were then centrifuged for $5 \mathrm{~min}$ at $1200 \mathrm{rpm}$ and the supernatant was discarded. Next, $5 \mu 1$ Human TruStain FcX ${ }^{\mathrm{TM}}$ in $100 \mu \mathrm{l}$ PBS/BSA was added and vortexed. The mixture was incubated for $10 \mathrm{~min}$ in the dark at RT. $100 \mu \mathrm{l}$ of antiVACV serum (1:50 or 1:100) in PBS/BSA were added. The mixture was incubated for $1 \mathrm{~h}$ in the dark at RT. $250 \mu \mathrm{l}$ PBS/BSA were added, vortexed and centrifuged for 5 min at $1200 \mathrm{rpm}$. Thereafter, anti-human IgG antibody was added in $100 \mu \mathrm{l}$ PBS/BSA according to the manufacturer's instructions. The mixture incubated for another hour in the dark at RT. Finally, cells were washed once in Perm Buffer and then resuspended in $50 \mu 1$ PBS/BSA. Cells were acquired by flow cytometry and data analyzed by the FlowJo software.

\subsubsection{Polychromatic flow cytometric calpox antigen staining of cells from buffy coat}

In order to also test granulocytes for the presence of calpox antigen, buffy coats were isolated as described (2.5 Isolation of peripheral blood mononuclear cells (PBMCs) and buffy coat from whole blood) and analyzed essentially as described for PBMCs (2.5 Isolation of peripheral blood mononuclear cells (PBMCs) and buffy coat from whole blood). Blood from healthy donor animals was included to assess background reaction (Table 2-7), because no autologous prevalues were available.

\subsubsection{T cell proliferation assay}

$\mathrm{T}$ cell proliferation assay was performed through labeling PBMCs with carboxyfluorescein succinimidyl ester (CFSE), and analyzing cell division. After isolation (2.4 Isolation of peripheral blood mononuclear cells (PBMCs)) PBMCs were stained with $100 \mu$ l CFSE $(10 \mu \mathrm{M})$ for $15 \mathrm{~min}$ at $37{ }^{\circ} \mathrm{C}$ and $5 \% \mathrm{CO}_{2}$. CFSE is a non-fluorescent and colourless cell staining dye, which passively diffuses into cells. It is covalently coupled with its succinimidyl group to intracellular amines and is therefore retained within the cell. CFSE acetate groups are then cleaved by intracellular esterases, which convert CFSE molecules into fluorescent esters. 100 $\mu 1$ FCS was added to stop the reaction. Cells were then washed in a centrifugation step at 350 $\mathrm{x} g$ for $8 \mathrm{~min}$ at room temperature. After the supernatant was discarded, cells were resuspended in $1 \mathrm{ml}$ PBMC cell culture medium (2.4 Isolation of peripheral blood mononuclear cells (PBMCs)). Subsequently, PBMCs were washed again (as decribed before), the supernatant was discarded and cell numbers were adjusted to $1.3 \times 10^{6}$ cells $/ \mathrm{ml}$ PBMCs.

One portion of the cells was left untreated as controls (RPMI), another portion was stimulated with $1 \%$ concanavalin A (ConA, Sigma) and a further portion with inactivated modified vaccinia virus Ankara at a multiplicity of infection of 3. Cells were seeded for each formulation 
in triplicate at a cell density of $2 \times 10^{5}$ cells per well of a U-bottom shaped 96-well plates in a total volume of $150 \mu \mathrm{l}$ RPMI medium Next, either $50 \mu \mathrm{l}$ of RPMI or the stimulating agents in the same volume were added and incubated at $37{ }^{\circ} \mathrm{C}$ and $5 \% \mathrm{CO}_{2}$ for six days. Cells were checked daily for proliferation using a microscope.

\section{Polychromatic flow cytometriy staining of the $\mathbf{T}$ cell proliferation assay}

After this stimulation period, cells were stained with a cocktail of fluorophore-labelled antibodies to assess proliferation of $\mathrm{T}$ cell subsets. This cocktail contained anti-CD8-PE, antiCD45RA-ECD, anti-CD28-PerCP-Cy5.5, anti-CD69-PE-Cy7, anti-CD95-APC, anti-CD3AF700, anti-CD4-V450, and Zombie Yellow and fixable Viability kit for live/dead staining. CFSE-stained cells from triplicates were pooled, transferred to FACS tubes and washed once with $250 \mu \mathrm{l}$ PBS/BSA. After centrifugation for $5 \mathrm{~min}$ at $1200 \mathrm{rpm}$, the supernatant was discarded the cell pellet resuspended in $250 \mu \mathrm{l}$ PBS/BSA, vortexed and the cells again centrifuged. Supernatant was discarded and the cells were stained with antibody mixture for $30 \mathrm{~min}$ in the dark at room temperature. All antibodies were used at pretitrated concentrations. Cells were then washed again with $250 \mu \mathrm{PBS} / \mathrm{BSA}$ and were resuspended in $50 \mu \mathrm{l} \mathrm{FA-solution.}$ Cells were acquired and analyzed (see 2.7.2 Polychromatic flow cytometric staining for calpox antigen in PBMCs).

\subsubsection{Polychromatic flow cytometric analysis of whole blood}

Polychromatic flow cytometric analysis of whole blood was performed in order to analyze the proportions of different adaptive and innate immune cells in the vaccination studies during immunization and after challenge.

The staining cocktail comprised the following antibody conjugates: anti-CD45-Biotin, antiy $\delta$ TCR-FITC, anti-CD3-AF700, anti-CD14- PerCP-Cy5.5, anti-CD16- APC-Cy7, anti¡ $\delta$ TCR-FITC, anti-CD123-PE, anti-CD11c-PE-Cy7, anti-CD86-APC, anti-CD11b-Pacific Blue, and, and a streptavidin-V500 conjugate. All antibodies were used at pretitrated concentrations. CD45-Biotin was preincubated with Streptavidin-V500 for $15 \mathrm{~min}$ (1:1000). Per test $50 \mu 1$ of the CD45-Biotin/Streptavidin mixture was added to $23.5 \mu 1$ of the mixture of the antibodies. This mixture was added to FACS tubes. $50 \mu$ l of whole blood were incubated with this mixture for $30 \mathrm{~min}$ in the dark at RT. $1 \mathrm{ml}$ of RBC Lysis/Fixation solution (1x) was added. The mixture was then vortexed and incubated for $15 \mathrm{~min}$ in the dark at RT. Cells were washed with $250 \mu \mathrm{PBS}$ PSA, after pelleting dissolved in $50 \mu \mathrm{PBS} / \mathrm{BSA}$, and acquired and analyzed. 
Results of the samples are given in \% cell proportions after baseline correction.

\subsubsection{Polychromatic flow cytometric staining of whole blood for the determination of absolute numbers of different immune cell subsets}

Absolute cell numbers of different immune cells were assessed by staining whole blood. For this approach the staining cocktail comprised the following antibody conjugates: anti-CD45Biotin, anti-CD3-AF700, anti-CD20-FITC, anti-CD8a-PE, anti-CD4-PerCP-Cy5, anti-CD69PE-Cy7, anti-CD159a-APC, anti-HLA-DR-APC-Cy7, anti-CD80-V450, anti-CD27-Brilliant Violet 650 , and a streptavidin-V500 conjugate. All antibodies were used at pretitrated concentrations. CD45-Biotin was preincubated with Streptavidin-V500 for $15 \mathrm{~min}$ (1:1000). $50 \mu 1$ of the CD45-Biotin/Streptavidin mixture per test was added to the mixture of the antibodies. This mixture was added to Trucount ${ }^{\mathrm{TM}}$ tubes. $50 \mu 1$ of whole blood was carefully added above the metal retainer and the tube was vortexed. Then, cells were incubated with the mixture of the antibodies for $15 \mathrm{~min}$ in the dark at RT. Next, $450 \mu \mathrm{l}$ of RBC Lysis/Fixation solution (1x) were added followed by vortexing followed by an incubation step for $15 \mathrm{~min}$ in the dark at RT. Each Trucount ${ }^{\mathrm{TM}}$ tube contains a known number of fluorescent beads, which are released when the lyophilised pellet is dissolved. Cells were acquired immediately after the incubation time and analyzed. The absolute number of positive cells (cells/ $\mu 1)$ in each sample was then determined by comparing cellular events to bead events. The number of positive cellular events was divided by the number of bead events and then multiplied by the respective Trucount ${ }^{\mathrm{TM}}$ bead concentration divided by the used blood volume $(50 \mu \mathrm{l})$.

\subsection{Virus quantification}

\subsubsection{Virus quantification by endpoint dilution assay in tissue homogenates}

Infectious calpox virus in tissue homogenates was determined by endpoint dilution assay. For this, $100 \mu 1$ of the tissue homogenate (2.6.2 DNA extraction from tissue samples) were used. The tissue homogenates were tenfold serially diluted from $10^{-1}$ to $10^{-4}$ with tissue DMEM medium. Viral dilutions were analysed in quadruplicate adding $100 \mu 1$ of each dilution to the pre-seeded Vero E6 cells in 96-well plates.

Plates were kept at $37{ }^{\circ} \mathrm{C}$ and $5 \% \mathrm{CO}_{2}$. The cell monolayer was washed three times with 100 $\mu \mathrm{l}$ PBS $24 \mathrm{~h}$ after inoculation and each well was supplemented with $100 \mu \mathrm{l}$ of the tissue DMEM medium.

Overall, the plates were incubates for four days at $37{ }^{\circ} \mathrm{C}$ and $5 \% \mathrm{CO}_{2}$. After that, intracellular immunostaining of the cells was performed. For permeabilization of cell membranes, the cells 
were treated with methanol for $15 \mathrm{~min}$ at $-20{ }^{\circ} \mathrm{C}$. Thereafter, plates were washed PBS three times. Unspecific binding was blocked by incubating the cells with $2 \%$ milk powder in PBS for $1 \mathrm{~h}$, at $37^{\circ} \mathrm{C}$. To detect intracellular calpox virus antigen, a human anti-VACV hyperimmune serum kindly provided by Prof. Czerny. The human anti-VACV serum was diluted in 1:100 in $2 \%$ milk powder in PBS, and $50 \mu \mathrm{l}$ were added to each well and incubated for $1 \mathrm{~h}$ at $37{ }^{\circ} \mathrm{C}$. After three washing steps with PBS an incubation with horseradish peroxidaseconjugated secondary antibody (peroxidase-conjugated AffiniPure $\mathrm{F}\left(\mathrm{ab}^{\prime}\right) 2$, $(\mathrm{H}+\mathrm{L})$-conjugated anti-goat anti-human IgG antibody) followed. The secondary antibody was 1:1000 diluted in $2 \%$ milk powder in PBS. $50 \mu \mathrm{l}$ were added to each well and incubated for $1 \mathrm{~h}$ at $37{ }^{\circ} \mathrm{C}$. The wells were then washed 3 times with PBS. Binding of conjugate to the calpox antigen was visualized by adding $50 \mu \mathrm{l}$ per well of a substrate containing $2 \mu \mathrm{g}$ AEC (3-Amino-9EthylCarbazole), $6 \mathrm{ml} / \mathrm{l} \mathrm{DMF}$ (Dimethylformamide), $0.25 \mu 13 \% \mathrm{H}_{2} \mathrm{O}_{2}$ (Hydrogen peroxide) and $1 \mathrm{ml} / \mathrm{l} 50 \mu \mathrm{l} \mathrm{NaOAc}$ (sodium acetate)) for $20 \mathrm{~min}$. The colour reaction resulted in a dark reddisch-brown intracytoplasmic staining which was evaluated unter the microscope. Results are given as $50 \%$ tissue culture infective dose $\left(\mathrm{TCID}_{50}\right) / \mathrm{g}$ tissue which were calculated according to Reed and Muench method (Fridholm\&Everitt, 2005; LaBarre\& Lowy, 2001)

\subsubsection{Virus quantification by endpoint dilution assay in buffy coat}

A plaque assay was conducted to detect replicating calpox virus in blood by cocultivation of buffy coat leucocytes with Vero E6 cells. To this end, $100 \mu 1$ of whole blood was used. Blood was centrifuged for $15 \mathrm{~min}$ at $2455 \mathrm{rpm}$. The upper phase containing the plasma was taken off and stored at $-20{ }^{\circ} \mathrm{C}$ for serological analyses. The phase under the plasma contained the buffy coat and was visible as a white layer. The buffy coat was washed in $1 \mathrm{ml}$ titration medium (Dulbecco's modified Eagle's medium (DMEM), 4.5 g/L glucose (PAN-Biotech) with $2 \%$ fetal calf serum (FCS) and $1 \%$ penicillin-streptomycin (P/S)), centrifuged for $5 \mathrm{~min}$ at 1200 rpm, and the supernatant discarded. Leukocytes were resuspended in $1 \mathrm{ml}$ titration medium corresponding to an initial 1:10 dilution of the cell suspension. Samples were then serially diluted in $\log 2$ steps up to 1:5120. $500 \mu \mathrm{l}$ of each dilution were added to pre-seeded Vero E6 cells in 12-well plates. As a control cells were incubated with $500 \mu 1$ of Vero E6 cell culture medium. The plates were incubated for three days at $37{ }^{\circ} \mathrm{C}$ and $5 \% \mathrm{CO}_{2}$. In order to remove unadsorbed virus, cell monolayers were washed $24 \mathrm{~h}$ after inoculation, and $500 \mu \mathrm{l}$ of fresh titration medium was added to each well.

Further processing followed the protocol decribed in paragraph 2.8.1 except that the required volume were adapted to the 12 -well plates. For the hyperimmune serum, the conjugated sec- 
ondar antibody and the substrate $500 \mu \mathrm{l}$ per well were used. Primary plaques were defined as the biggest plaques of the same size and counted under the microscope. The secondary comets were not counted. Results are given in plaque forming units per $\mu 1$ blood $[\mathrm{pfu} / \mu \mathrm{l}]$.

\subsection{Detection of calpox virus}

\section{Gating strategy for the detection of calpox virus antigen in immune cells}

The gating strategy for the identification of calpox infected innate and adaptive immune cells is depicted in Figure 2-4. In the first step, doublets were excluded from analysis by applying a singlets gate using forward scatter (FSC)-height (-H) against FSC-area (-A). Neutrophils, monocytes and lymphocytes as populations of leukocytes were analyzed based on side scatter (SSC)-area (-A) versus CD45 expression. Three different subsets of monocytes were classified by CD14 and CD16. Lymphocytes were characterized by CD3 and CD20 expression to distinguish between $\mathrm{CD}^{+} \mathrm{T}$ cells and $\mathrm{CD} 20^{+} \mathrm{B}$ cells as well as to identify $\mathrm{CD} 3^{+} \mathrm{CD} 20^{+}$cells. NK cells were analyzed by gating on the $\mathrm{CD} 3^{-} \mathrm{CD} 20^{-}$population, followed by gating on CD159a $\mathrm{a}^{+}$cells. Intracellular calpox antigen was visualized through an anti-VACV immune serum. 

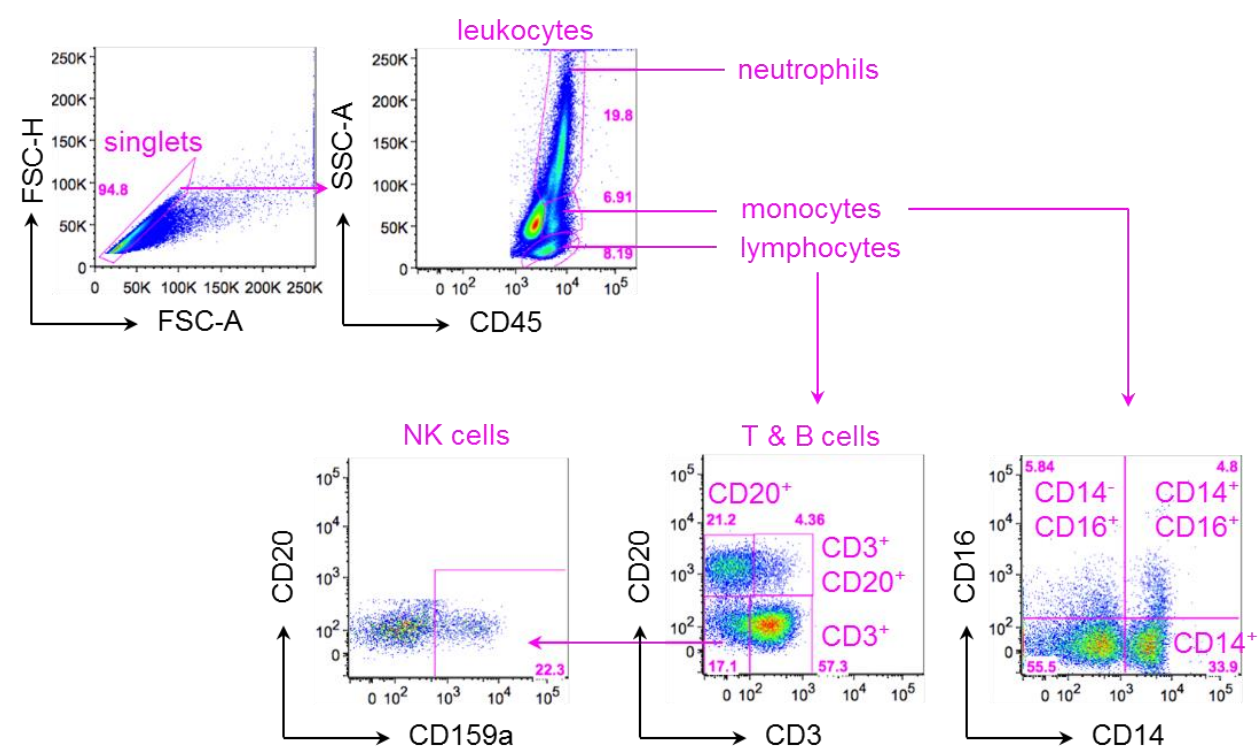

Figure 2-4. Gating strategy for the detection of calpox virus antigen in immune cells from buffy coat by flow cytometry. Representative gating strategy is depicted. Following exclusion of doublets lymphocytes, monocytes and neutrophils were gated on $\mathrm{CD} 45$ expression. Within the lymphocyte population $\mathrm{CD}^{+} \mathrm{T}$ cells and $\mathrm{CD} 20^{+} \mathrm{B}$ cells were distinguished. $\mathrm{NK}$ cells were defined as CD3- CD20- CD159a ${ }^{+}$. Monocytes were further distinguished based on CD14 vs. CD16 expression. Cell populations are demonstrated as dot plots. Pink lines, one gate; pink number, events within a gate given as percentages of the previous population.

\subsection{Quantitative real-time PCR}

A real-time PCR assay was used for the detection and quantification of calpox virus DNA (Table 2-9 and 2-10). The plasmid standard contained the target sequence and was used for viral DNA quantification. The amplicon of the target region of the "ankyrin repeat-containing protein" (CAM58389.1, target size of 78 bp) was cloned into a TOPO-TA vector (Invitrogen, Karlsruhe, Germany) which was kindly provided by Dr. Constanze Yue (RKI, Berlin, Germany). Plasmid DNA was quantified in NanoDrop photospectrometer and plasmid copy numbers were calculated. The real-time PCR quantification of all samples was performed in duplicate. Primers used are given in Table 2-9, real-time PCR cycling conditions and components in Table 2-10. Each real-time PCR run was analysed using the 5-Plex Rotor-Gene Q using the Rotor-Gene Q Series Software 2.1.0. The viral DNA quantities were expressed as calpox DNA copy numbers per $\mathrm{g}$ tissue. The detection limit was 50,000 calpox DNA copy numbers/g tissue. For blood the detection limit was 10 calpox DNA copies/ $\mu 1$ blood. The data of viral load in blood in the vaccination study I were acquired by Tingchuan Shi preliminarily to this thesis. 
Table 2-9: Oligonucleotides used in calpox virus-specific real-time PCR assay.

\begin{tabular}{l|l}
\hline Oligonucleotide name & Oligonucleotide sequence 5' $\rightarrow 3^{\prime}$ \\
\hline & Amplicon length: 78 bp \\
Calp1for & CCggCATgCgTgACTgAATT \\
Calp1rev & TAAgATgCgAgCCgAgAAgC \\
TIB-1 & FAM-TgCTCCgTgTTCTACCATCgTgCg-TMR \\
\hline
\end{tabular}

The base "G" is given as small letter to avoid confusion with "C"; FAM = 5'-Modification 6-FAM label; TMR = 3'Modification TAMRA label.

Table 2-10: Cycling conditions and components for calpox virus-specific real-time PCR.

\begin{tabular}{lll}
\hline Cycling conditions & \\
\hline $95^{\circ} \mathrm{C}$ & $10 \mathrm{~min}$ & \\
\hline $95^{\circ} \mathrm{C}$ & $15 \mathrm{sec}$ & 45 \\
$58^{\circ} \mathrm{C}$ & $45 \mathrm{sec}$ & repeats
\end{tabular}

\begin{tabular}{ll} 
Approach $15 \mu \mathrm{l}$ & \\
\hline $\mathrm{H}_{2} \mathrm{O}$ & $4 \mu \mathrm{l}$ \\
Primer "Calp 1 for" $100 \mu \mathrm{M}$ & $1 \mu \mathrm{l}$ \\
Primer "Calp 1 rev" $100 \mu \mathrm{M}$ & $1 \mu \mathrm{l}$ \\
Probe "TIB-1" $100 \mu \mathrm{M}$ & $0.5 \mu \mathrm{l}$ \\
TaqMan® Universal PCR Master Mix & $7.5 \mu \mathrm{l}$ \\
DNA & $1 \mu \mathrm{l}$
\end{tabular}

For detailed information about the oligo nucleotides, see Table 2-9.

\subsection{Serological analyses}

\subsubsection{Binding antibodies by enzyme-linked immunosorbent assay (ELISA)}

An ELISA was performed to analyse the humoral plasma response for binding antibodies against whole viral particles of vaccinia virus (VACV) Lister Elstree (LE) and calpox virus. Antibody levels of different plasma samples (2.1.4 Sampling, Plasma samples) were determined to identify immune correlates.

\section{Virus purification}

To harvest and concentrate VACV LE and calpox virus which was then to be used as antigen for coating 17 tissue culture flasks (T175) were cultured with Vero E6 cells in a volume of 45 $\mathrm{ml}$. The cells were infected with a sufficient amount of the respective virus at $95 \%$ confluency and harvested. The cells were inoculated with VACV LE for two days and with calpox virus three days. Each virus/cell suspensions (VACV LE and calpox virus) were frozen at -80 ${ }^{\circ} \mathrm{C}$. Cell culture material was then thawed and centrifuged in a Sorvall centrifuge (Thermo Scientific, Sorvall® RC-5B Refrigerated Superspeed Centrifuge; GS-3 rotor) at $9000 \mathrm{rpm}$ for 
$2 \mathrm{~h}$, at $4{ }^{\circ} \mathrm{C}$. The supernatant was discarded and the pellet resuspended in $40 \mathrm{ml} 1 \mathrm{mM}$ TRIS buffer, $\mathrm{pH}$ 9.0. Next, the resuspended cell debris was incubated for $30 \mathrm{~min}$ and then frozen at $-80{ }^{\circ} \mathrm{C}$.

The next day the suspension was thawed in a waterbath at $37{ }^{\circ} \mathrm{C}$, refrozen in liquid nitrogen and thawed again. The suspension was vortexted and treated thrice with a SONOPLUS ultrasound-homogenizer for one min followed by a centrifugation at $3218 \mathrm{rpm}$ for $10 \mathrm{~min}$, at $4{ }^{\circ} \mathrm{C}$ (Thermo Scientific, Heraeus, Multifuge 3S-R; 6446 rotor). Thereafter, the supernatant was transferred to a new falcon tube and purified with a sucrose cushion. To this end, as a first step purification was done with $10 \mathrm{ml}$ of $36 \%$ sucrose. A centrifugation step at $25000 \mathrm{rpm}$ for 90 min followed at $4{ }^{\circ} \mathrm{C}$ (Thermo Scientific, Sorvall Combi Plus, DuPont, Sorvall Ultraspeed Centrifuge, Sorvall AH0-629 Rotor $29000 \mathrm{rev/min} \mathrm{MAX;} \mathrm{SW} 28$ rotor). The pellet was resuspended in $200 \mu 11 \mathrm{mM}$ TRIS buffer, pH 9.0 and incubated overnight at $4{ }^{\circ} \mathrm{C}$. The pellet was then again resuspended and transferred into a $1.5 \mathrm{ml}$ E-cup. In the next step a sucrose gradient was prepared with a gradient mixer. For that, $20 \%$ and $60 \%$ sucrose and $1 \mathrm{mM}$ TRIS buffer, pH 9.0 were used. The pellet was resuspended, vortexed and treated thrice with a SONOPLUS ultrasound-homogenizer for $1 \mathrm{~min}$. After that, the virus was layered onto the sucrose gradient and a centrifugation step at $15000 \mathrm{rpm}$ of $90 \mathrm{~min}$ at $4{ }^{\circ} \mathrm{C}$ followed (Beckmann Coulter Centrifuge, L-60 Ultraspeed Centrifuge; SW 40 rotor). The virus was visible as a band in the centrifugation tube and aspirated with a syringe by puncturing the tube. To eliminate the sucrose the concentrated virus suspension was then washed with $1 \mathrm{mM}$ TRIS buffer, pH 9.0 in a centrifugation step at $25000 \mathrm{rpm}$ of $1 \mathrm{~h}$, at $4{ }^{\circ} \mathrm{C}$ (Beckmann Coulter Centrifuge, L60 Ultraspeed Centrifuge; SW 40 rotor). The pellet was incubated with $100 \mu 11 \mathrm{mM}$ TRIS, pH 9.0 at $4{ }^{\circ} \mathrm{C}$ overnight. The following day, the pellet was resuspended and aliquoted into cryotubes. After a protein determination according to LOWRY the aliquots were stored at -80 ${ }^{\circ} \mathrm{C}$.

\section{Enzyme-linked immunosorbent assay (ELISA)}

The respective antigen (VACV LE or calpox virus) was bound to 96-well MaxiSorp ${ }^{\text {TM }}$ NuncImmuno $^{\mathrm{TM}}$ plates in a first coating step. For this, $100 \mu \mathrm{l} / \mathrm{well}$ of $5 \mu \mathrm{g} / \mathrm{ml}$ whole virus in coating buffer $\left(\mathrm{Na}_{2} \mathrm{CO}_{3}\right.$ buffer) were added to each well. Plates were closed with a sealing foil. After an incubation period of $24 \mathrm{~h}$ at $4{ }^{\circ} \mathrm{C}$, plates were washed three times with $200 \mu \mathrm{l} /$ well of PBS. The plates were then treated with $100 \mu \mathrm{l} /$ well of blocking buffer for $2 \mathrm{~h}$ at $37^{\circ} \mathrm{C}$ and $5 \%$ $\mathrm{CO}_{2}$ to prevent unspecific binding of antibodies. Plasma samples were diluted 1:200 in blocking buffer and $100 \mu \mathrm{l} /$ well were added in dublicate. 
Plasma samples of each animal obtained prior to vaccination were used to calculate the cutoff. Plates were closed with a sealing foil and an incubation step for $1 \mathrm{~h}$ at $37^{\circ} \mathrm{C}$. Plates were then washed five times with PBS. For detection of the primary antibody, the peroxidaseconjugated AffiniPure F(ab') $)_{2}$ Fragment Goat Anti-human $\operatorname{IgG}(\mathrm{H}+\mathrm{L})$ antibodies were diluted 1:1000 in blocking buffer and $100 \mu \mathrm{l} /$ well was added to the plates. Plates were sealed followed by incubation for $1 \mathrm{~h}$ at $37^{\circ} \mathrm{C}$. Plates were then washed ten times with PBS to eliminate unbound secondary antibodies. In the next step, $100 \mu \mathrm{l} /$ well TMB-substrate solution (3,3',5,5'-tetramethylbenzidine) was added and incubated for $20 \mathrm{~min}$ at room temperature in the dark. The reaction was stopped by adding $50 \mu \mathrm{l} /$ well Stop solution. Optical density was then measured using a Tecan Sunrise ${ }^{\mathrm{TM}}$ photometer equipped with the Magellan 6.4 Software. The samples were measured at $450 \mathrm{~nm}$ with a reference wavelength of $630 \mathrm{~nm}$. A plasma sample with a known antibody status (anti-MVA rabbit 1987, kindly provided by Prof. Czerny) served as positive control. 8 wells per plate were used as no template controls.

Twice the OD value determined for each pre-immunization plasma was used to define the individual cut-off value and was subtracted from the values from immune plasma.

\subsubsection{B cell epitope fine mapping by peptide microarray}

\subsubsection{Poxvirus peptide microarray layout}

The poxvirus peptide microarray was used to detect antibodies against linear epitopes of different orthopoxvirus antigens.

15mer peptides overlapping by twelve amino acids were synthetised via SPOT technique (Frank, 1992), passed through the SC process (Dikmans, 2006) and spotted onto microscope glass slides. The spot size was between $200-250 \mu \mathrm{m}$ and the peptide concentration in each spot was 360 fmol.

Six proteins of VACV Western Reserve i.e. A27, A33, B5, D8, H3 and L1 were selected and used for synthesis of 475 peptides covering the entire proteins (Figure 2-5B).

The GenBank accession numbers are as follows: A27 (YP_233032.1), A33 (YP_233038), B5 (YP_233069.1) D8 (YP_232995.1), H3 (YP_232983.1) and L1 (YP_232970.1).

The mature virion (MV) membrane-associated proteins (A27, D8, H3 and L1) and transmembrane proteins that are exposed on the outer surface of the enveloped virion (EV) (A33 and B5) were chosen because these determinants play a role in neutralization (Moss, 2011).

In addition, ten cellulose-conjugated biotin spots served as positive controls and for orienting the Spot Calling program. Each chip contained eight identical arrays (Figure 2-5, A) with the 
same arrangement of the spots on each array (Figure 2-5, B). An exemplary reaction of the first three peptides covering A27 and its respective amino acid sequence corresponding to spots \#1-\#3 in B is demonstrated (Figure 2-5, C).

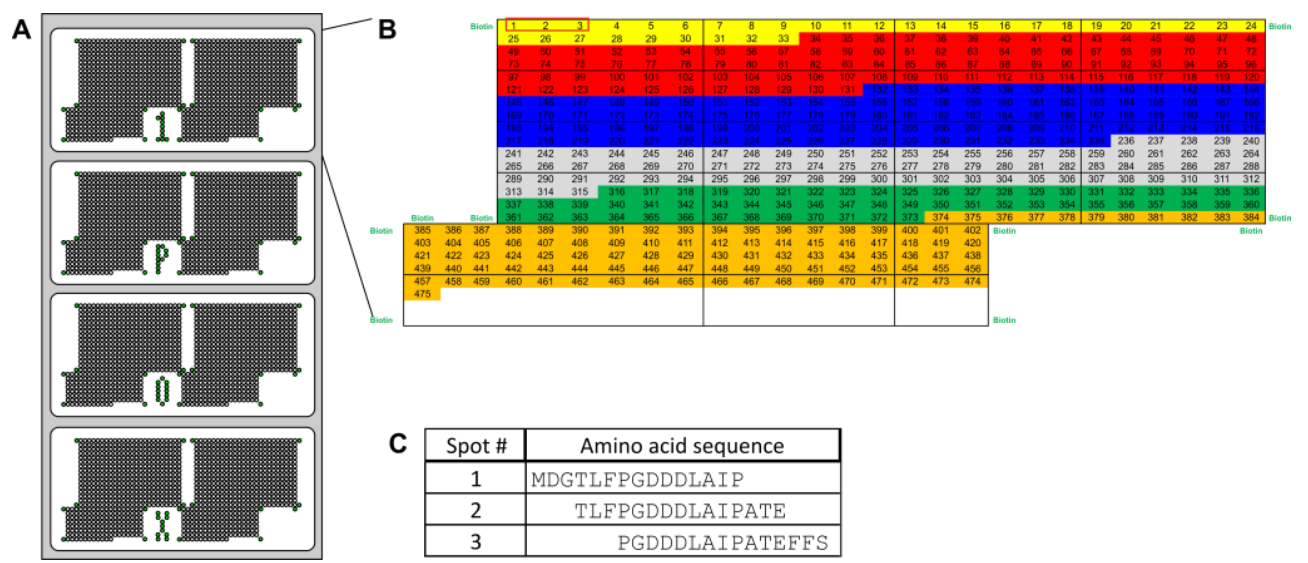

Figure 2-5: Layout of the poxvirus microarray chip. (A) The chip contained eight arrays arranged in the same manner. (B) Arrangement of spots on each array: yellow represents peptides of A27 antigen of Vaccinia virus Western Reserve; red, D8; blue, H3; grey, L1; green, A33 and orange, B5. The number serves as the batch identification and letters represent the chip name. (C) Amino acid sequence of the first three peptides covering A27, corresponding to spots \#1-\#3 in B (boxed in red).

\subsubsection{Microarray chamber}

The microarray chamber (Figure 2-6) was supposed to be used for the incubation of the microarray chips and was designed to analyze four microarray chips simultaneously. A homemade microarray chamber was used (Hotop, 2014) to avoid leakage and for multiple analysis in a humidified system. It was slighty modified in this study. The D-197 metal springs enabled a consistent pressure between the lid and the bottom that contains the microarray chips. To increase the pressure a $1 \mathrm{~mm}$ thick rubber cover (Figure 2-7) of 65 shore was glued with a special adhesive SB 2444 (Henkel AG \& Co. KGaA, Düsseldorf, Germany) to the lid.

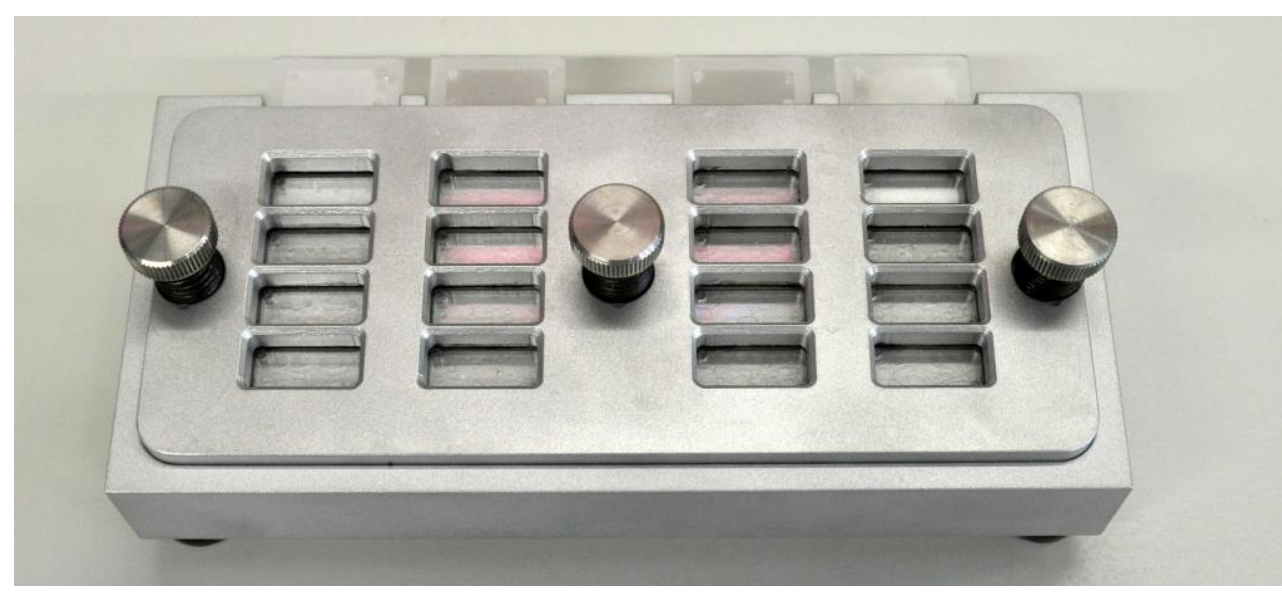

Figure 2-6: The poxvirus microarray chamber was designed to analyze four microarray chips simultaneously. Four microarray chips were placed into the metal chamber and the first antibody mixture that contained the plasma samples was added to the microarray chip. 


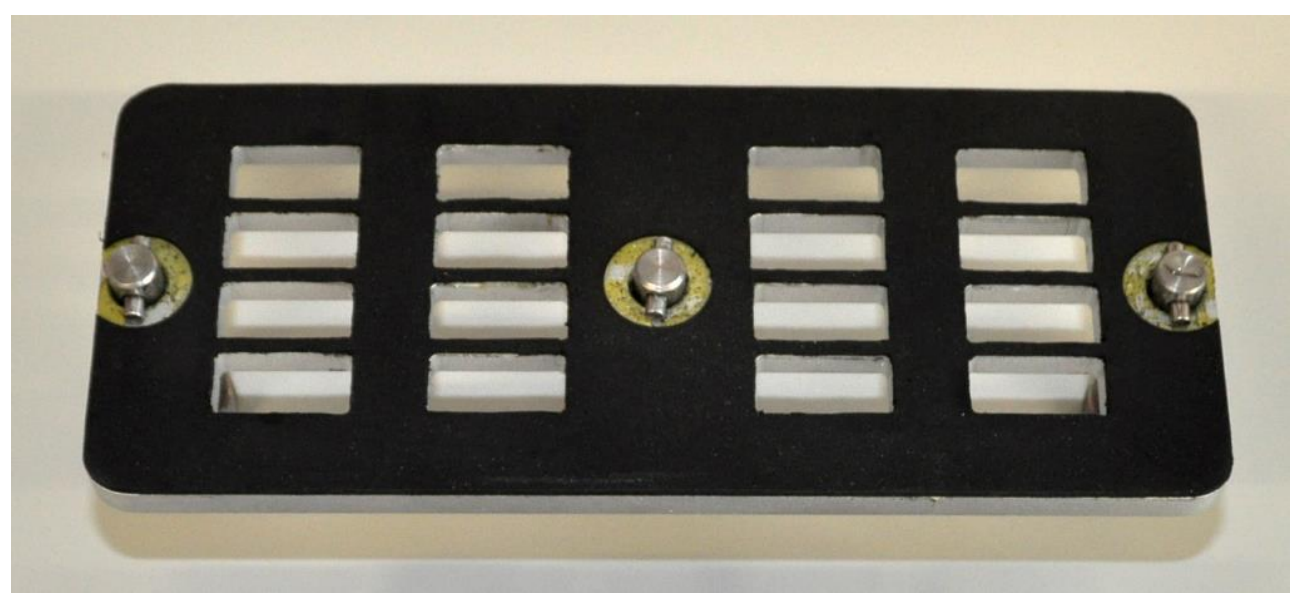

Figure 2-7: Rubber cover of the poxvirus microarray chamber's lid depicted from below.

\subsubsection{Validation of the chip using mAbs against known epitope regions}

The poxvirus peptide microarrays were pretested to evaluate the reactions by using monoclonal antibodies (mAbs) against a known epitope. The screening procedure of the microarray chip was performed as previously reported with few modifications (Hotop et al., 2014). Briefly, the microarray chip was washed with absolute ethanol for $3 \mathrm{~min}$. It was then washed with Tris-buffered saline (TBS) thrice for 3 min. The blocking step was done with blocking buffer using $2 \%$ casein in $0.05 \%$ Tween TBS (T-TBS) for $60 \mathrm{~min}$, at room temperature to block unspecific reactions. Then it was washed with T-TBS three times, each for 3 min. Protein concentrations were measured using NanoDrop ${ }^{\circledR}$ ND-1000 Spectrophotometer (NanoDrop Technologies, Inc., Wilmington, DE, USA). Next, the monoclonal antibody was diluted to 20 $\mathrm{mg} / \mu \mathrm{l}$ in the blocking buffer. Then $60 \mu \mathrm{l}$ of the diluted antibody were placed onto the chip. The poxvirus microarray chip was designed to screen 4 samples simultaneously in repeated determination, using an adhesive chamber (SecureSeal ${ }^{\mathrm{TM}}$, Sigma-Aldrich Co. LLC, USA) because the incubation had to be done under humidified conditions. Additionally, a microarray chamber, homemade press tool (2.11.2.2 Microarray chamber) was used to avoid leakage. After incubation of the antibody was incubated overnight in a cooling room at $4{ }^{\circ} \mathrm{C}$, three washing steps were performed with T-TBS for $5 \mathrm{~min}$. Next, $60 \mu \mathrm{l}$ of the secondary antibody mixture containing 1:240 diluted Cy3-conjugated streptavidin and Cy5-conjugated goat antimouse IgG (Jackson Immunoresearch Laboratories, West Grove, PA, USA) was added onto the chip together with an object plate.

After an incubation of the chip for $90 \mathrm{~min}$ at room temperature in a humidified chamber it was washed two times with T-TBS, three times with distilled water, each for $5 \mathrm{~min}$. The chip was then dried using compressed air and immediately analyzed with an Agilent DNA microarray scanner (Agilent Technologies, USA). 


\subsubsection{Poxvirus scanning chip in the calpox virus/marmoset model}

Samples from the vaccination studies I and II were tested with the optimized protocol. The blocking buffer was $2 \%$ casein in $0.05 \%$ Tween TBS (T-TBS) with $5 \%$ Albumin Fraction $\mathrm{V}$. The first antibody solution contained the plasma sample diluted 1:60 in blocking buffer. The mixture of secondary binding reagents contained 1:240 diluted Cy3-conjugated streptavidin and Alexa Fluor ${ }^{\circledR}$ 647-conjugated AffiniPure $\mathrm{F}\left(\mathrm{ab}^{\prime}\right)_{2}$ fragment goat anti-human IgG $(\mathrm{H}+\mathrm{L})$ (Jackson Immunoresearch Laboratories, West Grove, PA, USA). All other steps were identical to those described in the previous paragraph.

\subsubsection{Screening procedure and data acquisition of the microarray chip}

The microarray chips were scanned with an Agilent DNA microarray scanner (Agilent Technologies, USA). The true signals were identified by the semi-automated Spot Calling method (Hotop et al., 2014) as followed: after screening, the obtained photo was first saved as a TIFF file. The fluorescence intensity of each spot was calculated using the ImageJ Software (Schneider et al., 2012). Two times the averages of the sum of fluorescence intensities from all spots plus one standard deviation were calculated and used as cut off using Excel (Microsoft, version 2010).

\subsubsection{Plaque reduction neutralization test (PRNT)}

\section{Staining reagents and antibodies}

Crystal Violet Sigma-Aldrich C3886-25G; 077K07401 Crystal Violet

anti-MVA rabbit kindly provided by Prof. Czerny, Microbiology and animal

immunserum; $\quad$ hygiene, Georg-August University Göttingen

11.06.1997

To analyze the anti-poxvirus neutralization capacity of the different plasma samples of vaccination study I and II, a microtiter plaque reduction neutralization test (PRNT) was established because of the small amounts of plasma available from each animal. First, $3 \times 10^{4}$ Vero E6 cells/well were seeded into 96-well plates with DMEM and incubated for approximately $24 \mathrm{~h}$ at $37{ }^{\circ} \mathrm{C}$ and $5 \% \mathrm{CO}_{2}$. The cells should be $70 \%$ confluent at the start of the test for a good read-out. Plasma samples were serially diluted with DMEM and $100 \mu 1$ were mixed

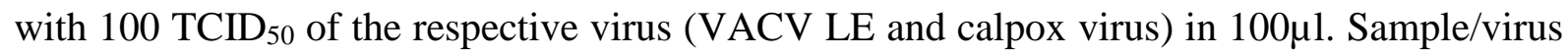
mixture incubated for $1 \mathrm{~h}$ at $37^{\circ} \mathrm{C}$ and was then added to the Vero E6 cells. After adsorbance for $1 \mathrm{~h}$ at $37{ }^{\circ} \mathrm{C}$ and $5 \% \mathrm{CO}_{2}$ plates were washed twice with PBS. Cells were then incubated in $200 \mu \mathrm{l} \mathrm{DMEM}$ at $37{ }^{\circ} \mathrm{C}$ and $5 \% \mathrm{CO}_{2}$ for $72 \mathrm{~h}$. After that, medium was removed and plates 
were stained with $100 \mu \mathrm{l}$ crystal violet $(1.5 \%$ in $80 \%$ ethanol)/well for 2 min and finally washed with $\mathrm{H}_{2} \mathrm{O}$. The plaques per well were then counted. When $50 \%$ plaque reduction compared to the virus control was observed, the reciprocal of the respective dilution was considered the neutralizing titre. The average result of a duplicate was determined. Anti-MVA rabbit immunserum was used as positive control (rabbit hyperimmune serum, anti-MVA antibody was kindly provided by Claus Peter Czerny).

\subsubsection{Gating strategy for the identification of innate and adaptive immune cells}

Gating strategy for the identification of innate and adaptive immune cells is depicted in Figure 2-8. After gating on singlets, lymphocytes were analyzed by gating on SSC-A ${ }^{\text {low }} \mathrm{CD}^{4} 5^{+}$cells. $\gamma \delta \mathrm{T}$ cells and T cells were analyzed in the next step (expression of $\gamma \delta$-TCR vs. CD3).

To analyze monocytes and DCs, another gating scheme was used (Figure 2-8). Following exclusion of doublets (by a single gate) as well as $\gamma \delta \mathrm{T}$ cells and conventional T cells, macrophages (MQs; CD14- $\left.{ }^{-} D 16^{+}\right)$and monocytes $\left(\mathrm{CD} 14^{+}\right.$and $\left.\mathrm{CD} 16^{+}\right)$were gated. The $\mathrm{CD}^{-} 4^{-}$and CD16- population was further analyzed regarding CD11c and CD123 expression to differentiate between myeloid dendritic cells (mDCs; CD11c $\mathrm{c}^{+} \mathrm{CD} 123^{-}$) and plasmacytoid dendritic cells (pDCs; CD11 $\mathrm{c}^{-} \mathrm{CD} 123^{+}$). Expression of the activation marker CD86 was analyzed on monocytes and macrophages.

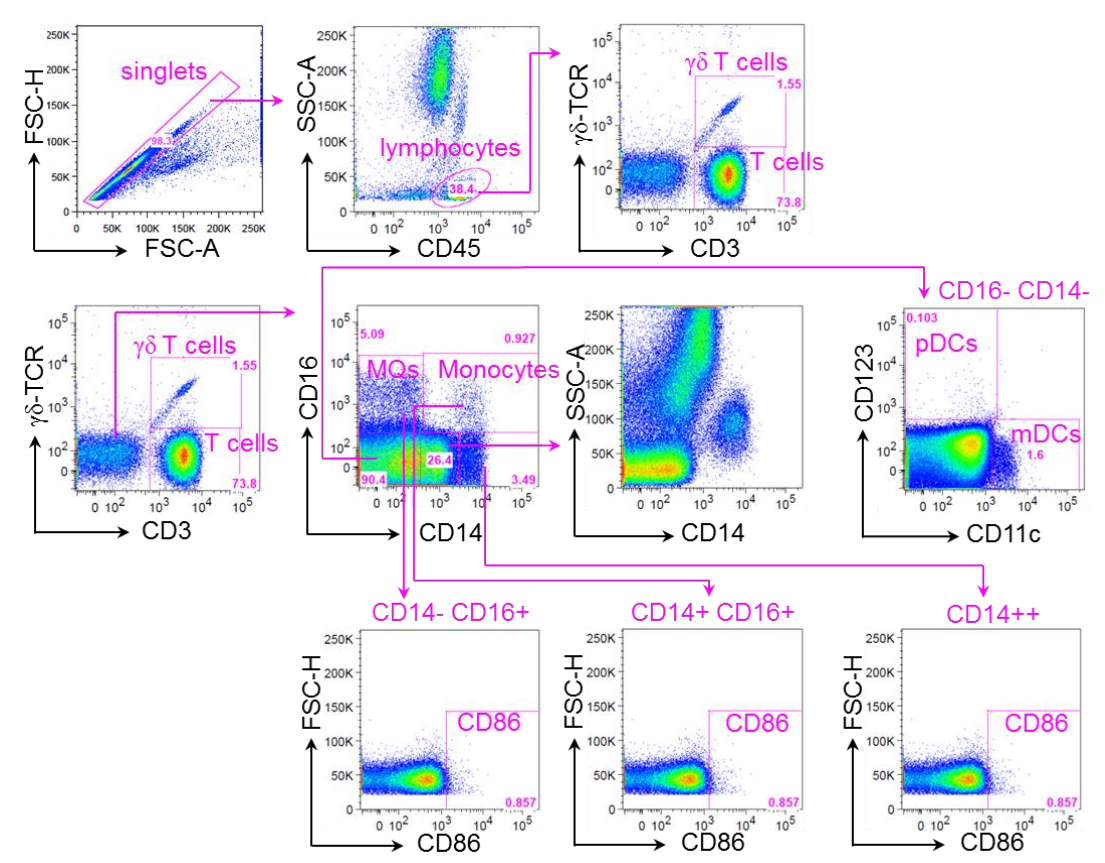

Figure 2-8. Gating strategy for the identification of innate and adaptive immune cells by flow cytometry. Representative dot plots of \#15095 are shown. Following exclusion of doublets lymphocytes were gated based on CD45 expression. $\gamma \delta$ $\mathrm{T}$ cells and $\mathrm{T}$ cells were then analyzed. Monocytes and DCs were analyzed using another gating scheme. Following exclusion of doublets as well as $\gamma \delta \mathrm{T}$ cells and conventional T cells, macrophages (CD14- CD16 ${ }^{+}$) and monocytes (CD14 ${ }^{+}$and $\mathrm{CD}^{+} 6^{+}$) were gated. The CD14- and CD16- population was analyzed based on CD11c and CD123 expression to differentiate between myeloid dendritic cells $\left(\mathrm{CD} 11 \mathrm{c}^{+} \mathrm{CD} 123^{-}\right)$and plasmacytoid dendritic cells $\left(\mathrm{CD} 11 \mathrm{c}^{-} \mathrm{CD} 123^{+}\right)$. Expression of the 
activation marker CD86 was analyzed on monocytes and macrophages and CD14 ${ }^{++}$cells. Pink lines, one gate; pink number, events within a gate given as percentages of the population before.

\subsubsection{Gating strategy to assess absolute cell counts of innate and adaptive immune cells}

The gating strategy for the identification of absolute counts of innate and adaptive immune cells is depicted in Figure 2-9. First, doublets were excluded by gating on singlets. Thereafter, lymphocytes were analyzed by gating on side scatter SSC-A against CD45 expression. CD3 ${ }^{+}$ $\mathrm{T}$ cells and $\mathrm{CD} 20^{+} \mathrm{B}$ cells were analyzed in the next step. T cells were divided into $\mathrm{CD} 4^{+}$, $\mathrm{CD}^{+}$and $\mathrm{CD}^{+} \mathrm{CD}^{+} \mathrm{T}$ cells and expression of the activation markers CD69 and HLA-DR (human leukocyte antigen - antigen D related). B cells were further analyzed for their expression of the activation marker CD80 and the memory marker CD27. NK cells were identified by gating on $\mathrm{CD}^{-} \mathrm{CD} 20^{-}$cells, followed by gating on $\mathrm{CD}^{+} \mathrm{CD}^{+} 159 \mathrm{a}^{+}$cells. Finally, CD69 and HLA-DR expression by NK cells and T cells was analyzed.
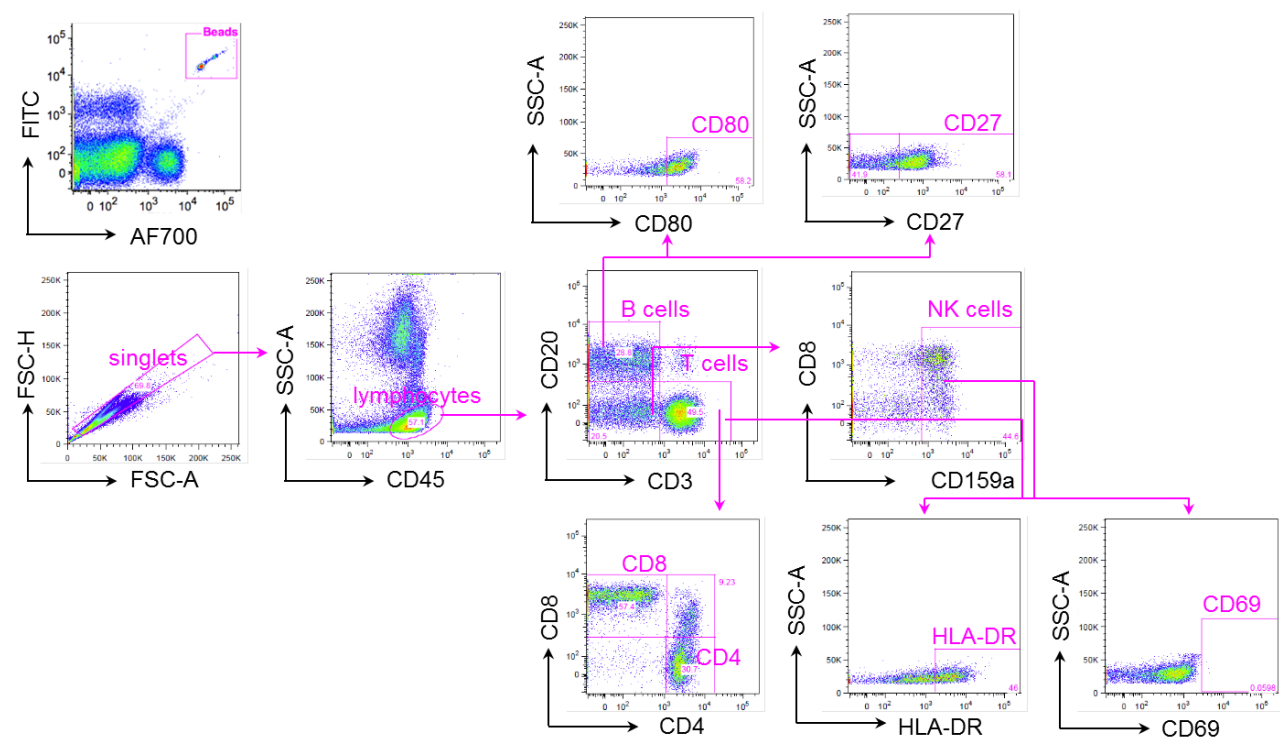

Figure 2-9. Gating strategy for the identification of absolute counts of innate and adaptive immune cells by flow cytometry. Representative dot plots of \#15095 are shown. To calculate absolute cell counts the number of cells of interest is put in relation to a defined amount of beads. The bead gate is set based on the fluorescence signal of FITC versus Alexa Fluor 700. Following exclusion of doublets lymphocytes were gated based on CD45 expression. $\mathrm{CD}^{+} \mathrm{T}$ cells and $\mathrm{CD} 20^{+} \mathrm{B}$ cells were then analyzed. $\mathrm{T}$ cells were divided into $\mathrm{CD} 4^{+}, \mathrm{CD} 8^{+}$and $\mathrm{CD} 4^{+} \mathrm{CD} 8^{+} \mathrm{T}$ cells and expression of the activation markers CD69 and HLA-DR. B cells were analyzed regarding expression of the activation marker CD80 and the memory marker CD27, NK cells were identified by gating on $\mathrm{CD}^{-} \mathrm{CD}^{-} 0^{-}$cells, followed by gating CD8 ${ }^{+/} \mathrm{CD} 159 \mathrm{a}^{+}$cells, CD69 and HLADR expression by NK cells was analyzed. Pink lines, one gate; pink number, events within a gate given as percentages of the population before.

\subsubsection{Gating strategy for $T$ cell proliferation assay}

The gating strategy is depicted in Figure $2-10 . \mathrm{CD}^{+} \mathrm{T}$ cell proliferation was analyzed by gating first on singlets (exclusion of doublets) by applying forward scatter FSC-A against FSC$\mathrm{H}$. Then gating was done on $\mathrm{CD} 3^{+} \mathrm{T}$ lymphocytes excluding dead cells. Next $\mathrm{CD} 4^{+}, \mathrm{CD}^{+}$ and $\mathrm{CD} 4^{+} \mathrm{CD} 8^{+}$cells (T cells) were identified. Stimulation of Callithrix jacchus $\mathrm{T}$ cells using 
concanavalin A (Con A) results in activation, proliferation and differentiation of both $\mathrm{CD}^{+}$ and $\mathrm{CD}^{+} \mathrm{T}$ cells and served as positive control. The proliferative activity of live $\mathrm{CD}^{+} \mathrm{T}$ cells treated with ConA, MVA or medium (negative control) were exemplified for one animal (\#14478) at 29 days post challenge (dpc).

CFSE$^{-}$cells represent the proliferating cells.
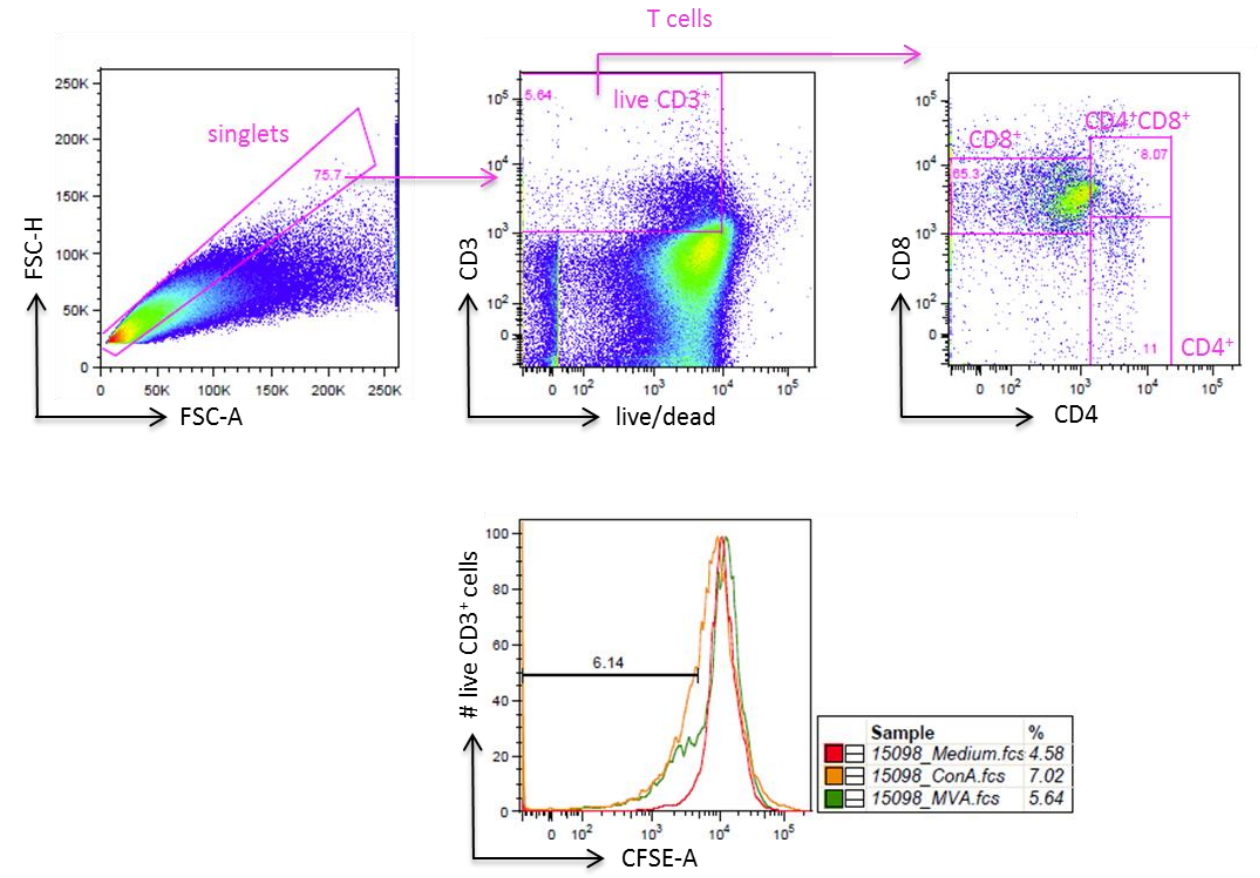

Figure 2-10. Representative gating strategy of CFSE-stained T cells from ConA or MVA-stimulated PBMCs from vaccination study II. PBMCs were labeled with the fluorescent dye CFSE to analyze T cell proliferation. PBMCs were either left untreated or stimulated with MVA or ConA as a control for 6 days. Subsequently PBMCs were stained for flow cytometric analyses. Representative dot plots from \#14478 are shown. Following exclusion of doublets, dead cells were excluded and $\mathrm{CD}^{+} \mathrm{T}$ cells were gated. These were further divided into $\mathrm{CD} 4^{+}, \mathrm{CD} 4^{+} \mathrm{CD} 8^{+}$and $\mathrm{CD} 8^{+} \mathrm{T}$ cells. Loss of CFSE signal was analyzed to evaluate proliferating cells (CFSE- cells). ConA, Concavalin A; MVA, modified vaccinia virus Ankara; pfu, plaque forming units; pink lines, one gate; pink number, events within a gate given as percentages of the population before.

\subsection{Statistical analyses}

Statistical analyses were performed with GraphPad Prism v5.0. The mean \pm standard deviation (SD) or median with range were determined. To detect significant differences in nonrelated samples, the non-parametric Mann-Whitney-U-test was used. For related samples, the paired t-test was used. The differences were considered significant when the significance level (p-value) was smaller than $0.05(*=\mathrm{p}<0.05 ; * *=\mathrm{p}<0.01 ; * * *=\mathrm{p}<0.001)$. 


\section{RESULTS}

\subsection{Pathogenesis study - portal of viral entry (I)}

\subsubsection{Detection of calpox virus in organs}

The aim was to determine the portal of viral entry by sacrificing infected animals in the presymptomatic phase at defined time points, i.e. 3, 5, 7, 10 and 12 days following inoculation. Therefore, a broad panel of different organs was analysed for the presence of calpox virus. The organs were analyzed for infectious calpox virus and calpox viral DNA.

\subsubsection{Replicating calpox virus and calpox DNA copies in different organs at dif- ferent time points after infection}

Infectious virus was quantified in tissues by endpoint dilution assay and compared to qRTPCR results using the same tissue preparation (Figure 3-1 and 3-2). Data generated by the two different analyses were normalized to one gram tissue. As it was difficult to anticipate at which time point after viral inoculation first traces of virus were detectable by the different virological techniques, first of all animals being inoculated for five days with the low viral dose were sacrificed. Actually, low levels of viral DNA ( $7 \times 10^{4}-4.7 \times 10^{6}$ copies/gram tissue) were found in two of the three animals in very few organs close to or representing the inoculation site, i.e. in one animal in the tonsil and in the other one in nasal mucosa and submandibular lymph node (Figure 3-1A), but no infectious virus was isolated from any of the tissues analysed. Next, animals inoculated with the same dose were euthanized at day 7, 10 and 12 . In the day-7-animals the data indicated a systemic spread of the virus. When merging the findings of all three animals of this group, all organs tested positive for viral DNA and from half of them infectious virus was detected. Notably, highest viral loads were observed in one animal in the nasal mucosa (animal 14483: $2.2 \times 10^{11}$ DNA copies and $2.3 \times 10^{8}$ TCID $_{50}$ ). Viral loads were considerably lower in the other organs ranging from approximately $10^{5}-10^{9}$ DNA copy numbers and $10^{2}-4 \times 10^{7}$ TCID50 (Figure 3-1B). At day 10, viral loads obviously had not increased compared to those observed in the animals sacrificed three days earlier. On this day of necropsy, however, infectious virus was more readily detectable in around $50 \%$ of the tissues. Levels of infectivity were either similar to viral DNA copies (animal 15149, nasal mucosa) or between one and five orders of magnitude lower than the copy numbers (Figure 31C). Altogether, highest viral levels were observed in the animals sacrificed at day 12 reaching up to $10^{16}$ copy numbers (animal 14702, nasal mucosa, bone marrow and adrenal gland) and up to $10^{9}$ infectious units (animal 14702, bone marrow). Remarkably, from each organ of 
mostly all three animals infectious virus was recovered at this late time point (Figure 3-1D). Taken into account the very low viral loads detected at day five following calpox infection thus rendering it unlikely to find any virus at an earlier time point, the viral dose was increased 40-fold to study infection events on day 3. In two of the three animals sacrificed at that day, viral DNA and infectious virus was found, one further animal remained negative for any viral parameter in any organ. Those two animals exhibited $6 \times 10^{8}-5 \times 10^{9}$ viral DNA copy numbers as well as considerable levels of infectious virus $\left(10^{5} \mathrm{TCID}_{50}\right)$ in the nasal mucosa. Moreover, in one of the two, tonsil, tongue lung, and eyes tested positive for calpox DNA, but copy numbers were by three or more orders of magnitude lower compared to the nasal mucosa $\left(\leq 10^{6}\right.$ copy numbers). Two thousandfold lower numbers of infectious virus $\left(\sim 50 \mathrm{TCID}_{50}\right)$ were recovered from tongue and esophagus when compared to the nasal mucosa. All the other organs remained negative for the two viral parameters (Figure 3-2).

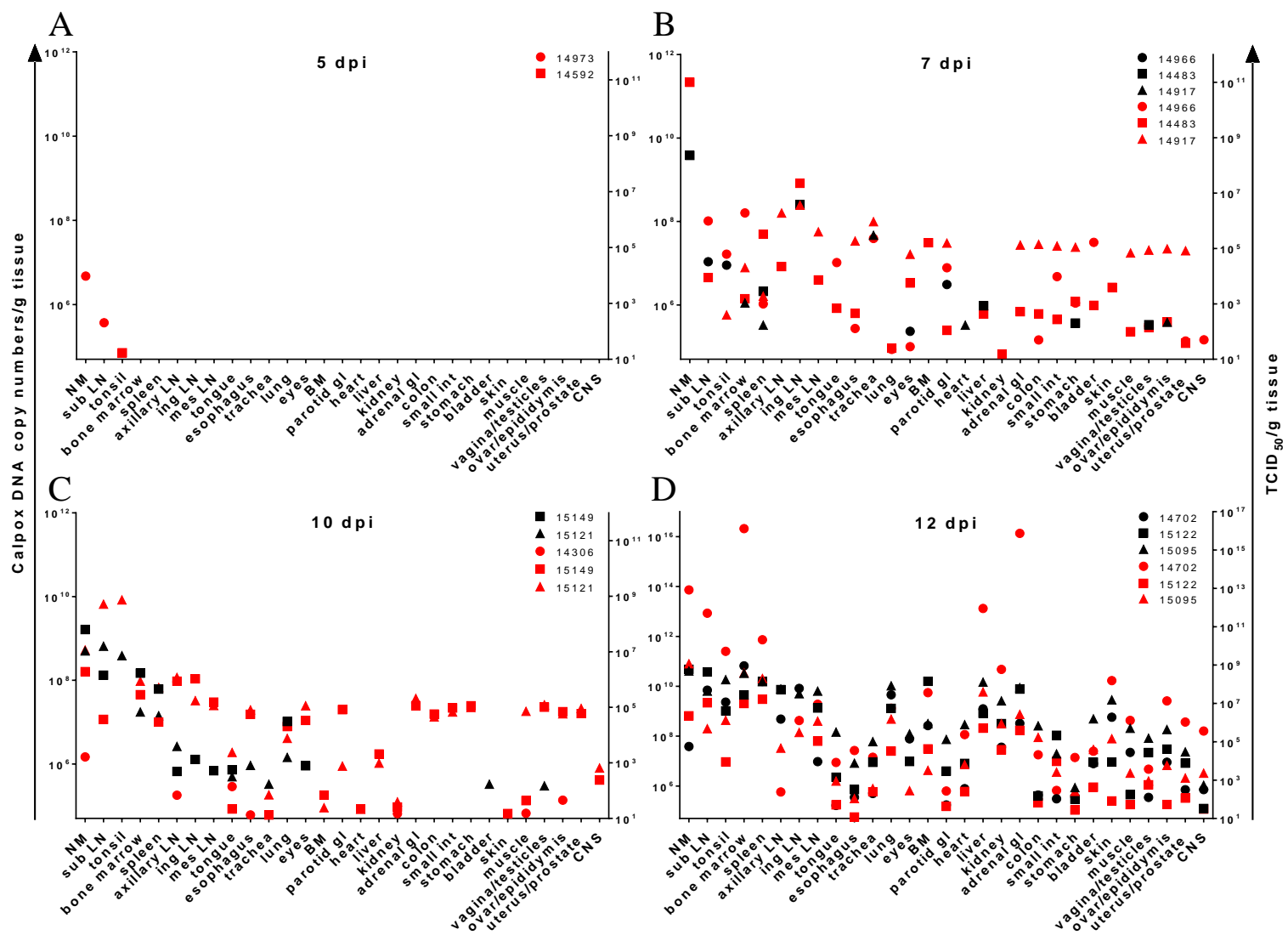

Figure 3-1. Calpox virus DNA copy numbers and TCID50 of infectious calpox virus in different tissues at different time points after i.n. infection. (A-D) Comparison of calpox DNA copy numbers per g tissue (red) and infectious calpox virus titre (black) at different time points after infection. Tissues are indicated at the x-axes. Calculation of the DNA copy numbers for calpox virus is based on the mean value of duplicate measurements and a plasmid standard for the real-time PCR assay. The detection limit is 50,000 calpox DNA copy numbers/g tissue. All day-5 animals were negative for infectious virus; BM, buccal mucosa; CNS, central nervous system; dpi, days post infection; gl, gland; ing, inguinal; int, intestine; LN, lymph node; mes, mesenteric; NM, nasal mucosa; sub, submandibular; TCID50, $50 \%$ tissue culture infective dose. 


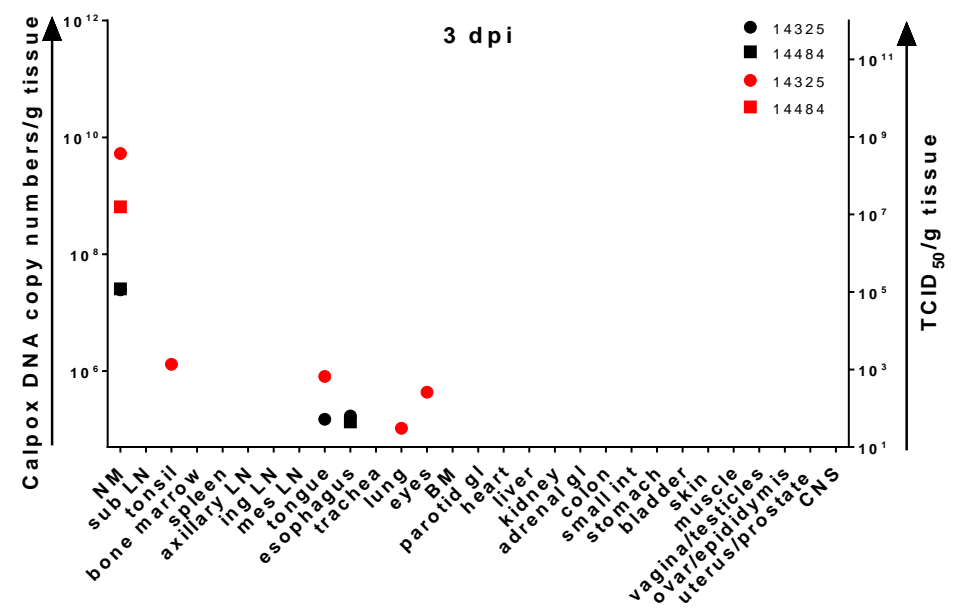

Figure 3-2. Calpox virus DNA copy numbers and TCID 50 of infectious calpox virus in different tissues three days after i.n. infection. Comparison of calpox DNA copy numbers per g tissue (red) and infectious calpox virus titre (black) at 3 dpi. Tissues are indicated at the x-axes. Calculation of the copy numbers for calpox virus is based on the mean value of duplicate measurements and a plasmid standard for the real-time PCR assay. The detection limit is 50,000 calpox DNA copy numbers/g tissue. One animal (\#14991) was negative in real-time PCR and plaque assay; BM, buccal mucosa; CNS, central nervous system; dpi, days post infection; gl, gland; ing, inguinal; int, intestine; LN, lymph node; mes, mesenteric; NM, nasal mucosa; sub, submandibular; TCID $\mathbf{5 0}, 50 \%$ tissue culture infective dose.

\subsection{Pathogenesis study - early dissemination and pathological sequelae of infec- tion (II)}

In the pathogenesis study the analysis of the early dissemination and pathological sequelae of infection (II) was in focus. The detection of the start of viremia and calpox virus antigens in immune cells were aims of the study.

\subsubsection{Detection of calpox virus in blood}

\section{The start of viremia as determined by real-time PCR is dose dependent}

Calpox viral DNA in blood was determined by real-time PCR at different time points after infection. Earliest calpox viral DNA in blood was detected in one of the two day-3 animals (\#14484) (Figure 3-3) which also was positive for viral DNA and infectious virus in organs close to the virus application site. Following inoculation with the lower viral dose, calpox viral DNA became first detectable in two of three animals at 7 dpi (\#14966 and \#14483) while all day-5 animals did not exhibit viremia. Unexpectedly, one of the day-10 animals remained calpox viral DNA negative in blood, although virus could be isolated from a number of organs. By contrast, all day-12 animals were viral DNA positive in blood exhibiting the highest copy numbers. The start of viremia is dose dependent since viremia was detected at 3 dpi with the high dose $\left(3.5 \times 10^{5} \mathrm{pfu}\right.$ calpox virus $)$ and at 7 dpi with the low dose $\left(8.3 \times 10^{3} \mathrm{pfu}\right.$ calpox virus). 


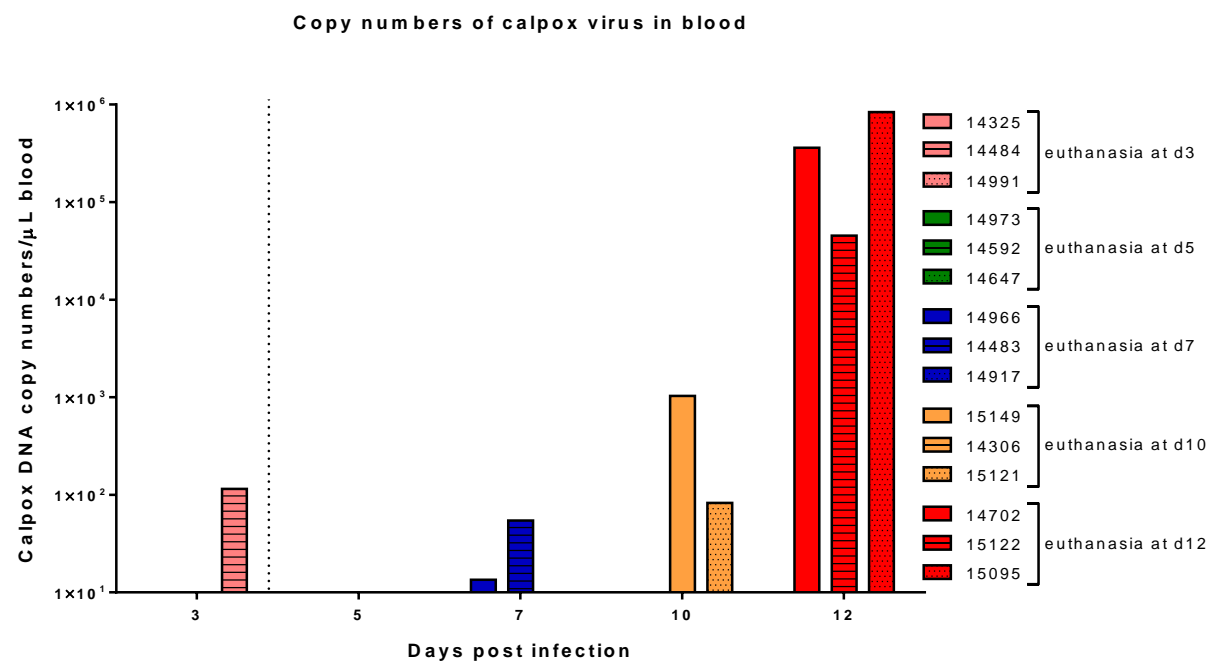

Figure 3-3. Calpox virus DNA in blood was determined by real-time PCR at the indicated time points after infection. d, day; The detection limit was 10 calpox DNA copy numbers/ $\mu 1$ blood.

First replicating virus was detected by cocultivation of buffy coat in one animal of the low-dose group at 7 days post infection (dpi)

To get an estimate on how calpox DNA copy numbers in blood translated into infectious virus, the calpox virus titre was determined by cocultivating leucocytes from buffy coat with Vero E6 cells. For this analysis, material was only available from the groups sacrificed on day 3 and day 12 after infection. In addition to the day of necropsy, blood from the day-12 group was also tested at days 3, 7, and 10. In contrast to the results from tissues, in the day-3 group no replicating virus was isolated from blood (Figure 3-4). First replicating virus was found in buffy coat of one animal (\#15095) from the day-12 group at 7 dpi. Infectious virus particles $[\mathrm{pfu} / \mu \mathrm{l}]$ were detected in all tested animals at $10 \mathrm{dpi}$ and at the last available time point at 12 dpi. The highest number of $3808.51 \mathrm{pfu} / \mu 1$ was reached in animal 15095 at $12 \mathrm{dpi}$. 

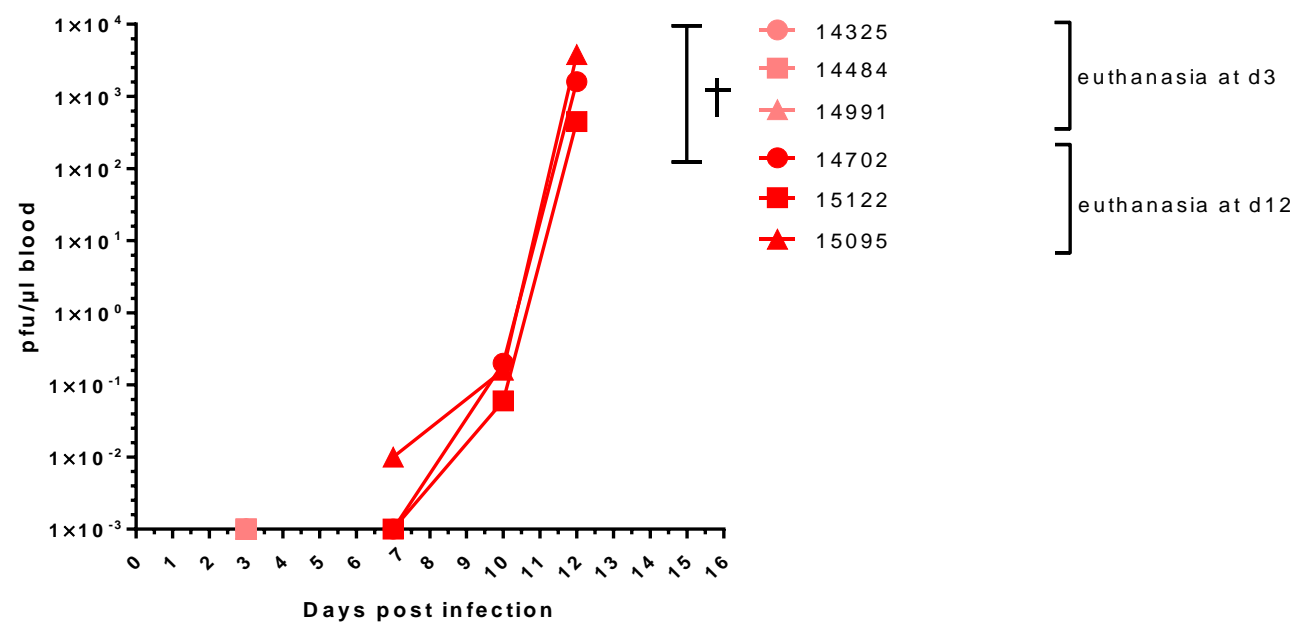

Figure 3-4. Replicating calpox virus in blood at different time points after infection by cocultivation of buffy coat with Vero E6 cells. First replicating virus (1/3) was detected in the low-dose group at 7 dpi. d, day; pfu, plaque forming units.

\subsubsection{Detection of calpox virus antigen in various immune cells by flow cytometry}

Although viremia was detectable either by PCR or limiting dilution assay, the infected cell type within the leucocytes could not be identified by these two techniques. To analyze which immune cells were infected, multicolour flowcytometry was used to differentiate between neutrophils, monocytes, T and B cells and NK cells. For this type of analysis again only blood from the groups sacrificed on day 3 and 12 after infection was available. The gating strategy is shown in the Materials and Method section (Figure 2-4).

\section{Calpox virus antigen positive (calpox ${ }^{+}$) cells were already detected in the day 3 group}

\section{infected with the high calpox dose}

Flowcytometric analyses revealed that calpox antigen was detected in the $\mathrm{CD} 3{ }^{+} \mathrm{CD} 20^{+}$lymphocyte subset in two out the three monkeys, in the classical monocytes (CD14 $\left.{ }^{+} \mathrm{CD} 16^{-}\right)$and in the intermediate monocytes $\left(\mathrm{CD} 14^{+} \mathrm{CD} 16^{+}\right)$of one of the day-3 animals (Figure 3-5, Figure 3$6)$.

In day-12 animals calpox antigen became readily detectable in all investigated immune cells at varying levels except for the neutrophils, which remained almost entirely negative for calpox virus antigen. Strikingly, all different immune cells of animal 15095 were positive for calpox protein. The animal 15095 was euthanized at the planned $12 \mathrm{dpi}$ and also for ethical reasons because of its moribund endpoint. In this animal the highest percentage of infected cells was observed in the $\mathrm{CD}^{+} \mathrm{CD} 20^{+}$cell population (almost $100 \%$ ), followed by around 75 to $90 \%$ infection of $\mathrm{B}$ cells and the different monocyte subtypes. $\mathrm{CD}^{+} \mathrm{T}$ cells were infected by $40 \%$ and NK cells by $55 \%$. The other two animals presented with a varying pattern of 
infection in the different cell types. However, calpox virus antigen was preferentially observed in the $\mathrm{CD} 3^{+} \mathrm{CD} 20^{+}$subset and the monocytes.

The results regarding the detection of calpox ${ }^{+}$cells in PBMCs were similar to the results of buffy coat. The courses were identical to the results in buffy coat, but the values in the controles were notably higher. Thus, only data from buffy coat staining are shown.

In summary, calpox virus infected in the initial phase preferentially $\mathrm{CD}^{+} \mathrm{CD} 20^{+}$and $\mathrm{CD} 14^{+}$ cells, and in the later phase also NK cells and B cells.

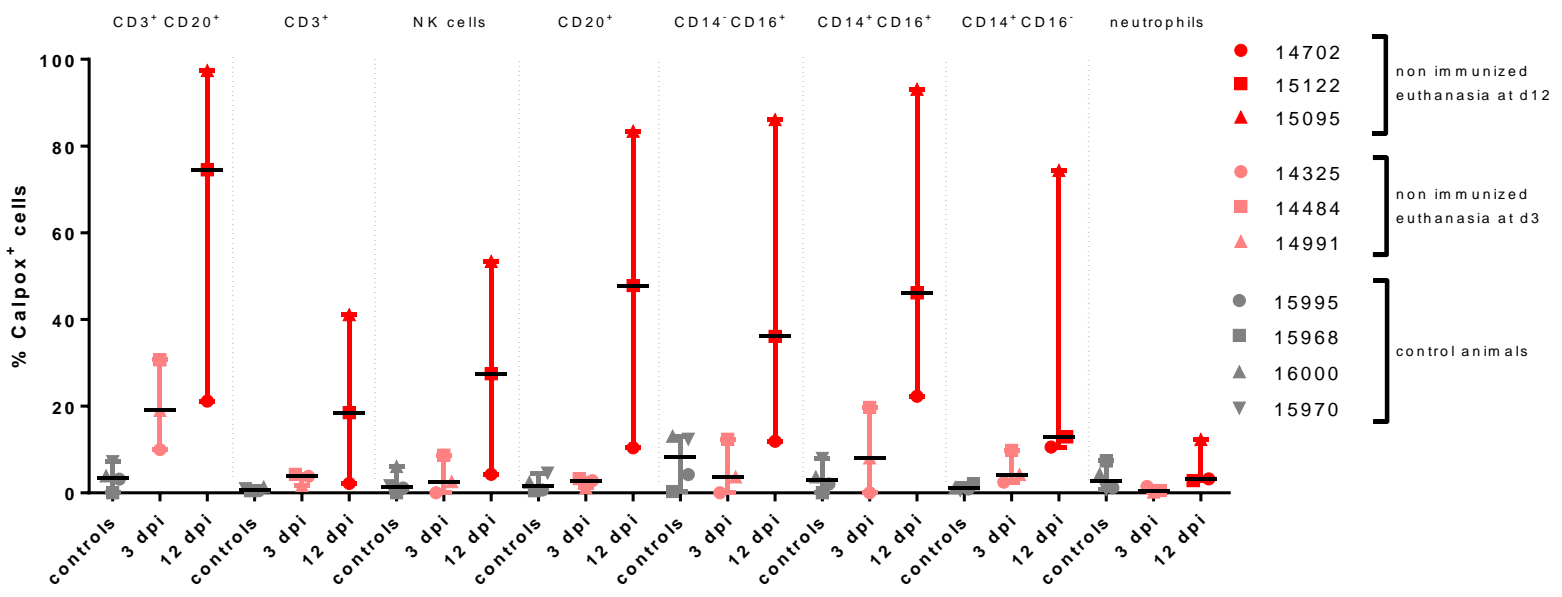

Figure 3-5. Calpox virus antigen was detected in immune cells of buffy coat by flow cytometry. The day- 3 animals received a higher dose $\left(3.5 \times 10^{5}\right.$ pfu calpox virus) than the animals euthanized on day 12 ( $8.3 \times 10^{3}$ pfu calpox virus). Medians with the range of percent calpox antigen positive $\left(\right.$ calpox $\left.^{+}\right)$stained cells are shown. Calpox ${ }^{+}$stained cells of the control animals were used to determine the baseline level. Calpox $^{+}$cells of the day- 3 animals were close to the calpox ${ }^{+}$cells of naïve baselines, except for calpox ${ }^{+}$cells of $\mathrm{CD}^{+} \mathrm{CD} 20^{+}$cells and $\mathrm{CD} 14^{+} \mathrm{CD} 16^{+}$monocytes. Levels of calpox ${ }^{+}$cells in the day- 12 animals were all higher than the calpox ${ }^{+}$cells of baselines except for the neutrophils. Calpox ${ }^{+}$cells of $\mathrm{CD}^{+}$cells and calpox ${ }^{+}$ cells of $\mathrm{CD} 14^{+} \mathrm{CD} 16^{-}$cells were low at $12 \mathrm{dpi}$. The cut off was defined as the highest control value of each of the respective cells. dpi, days post infection; Calpox ${ }^{+}$, calpox virus antigen positive.
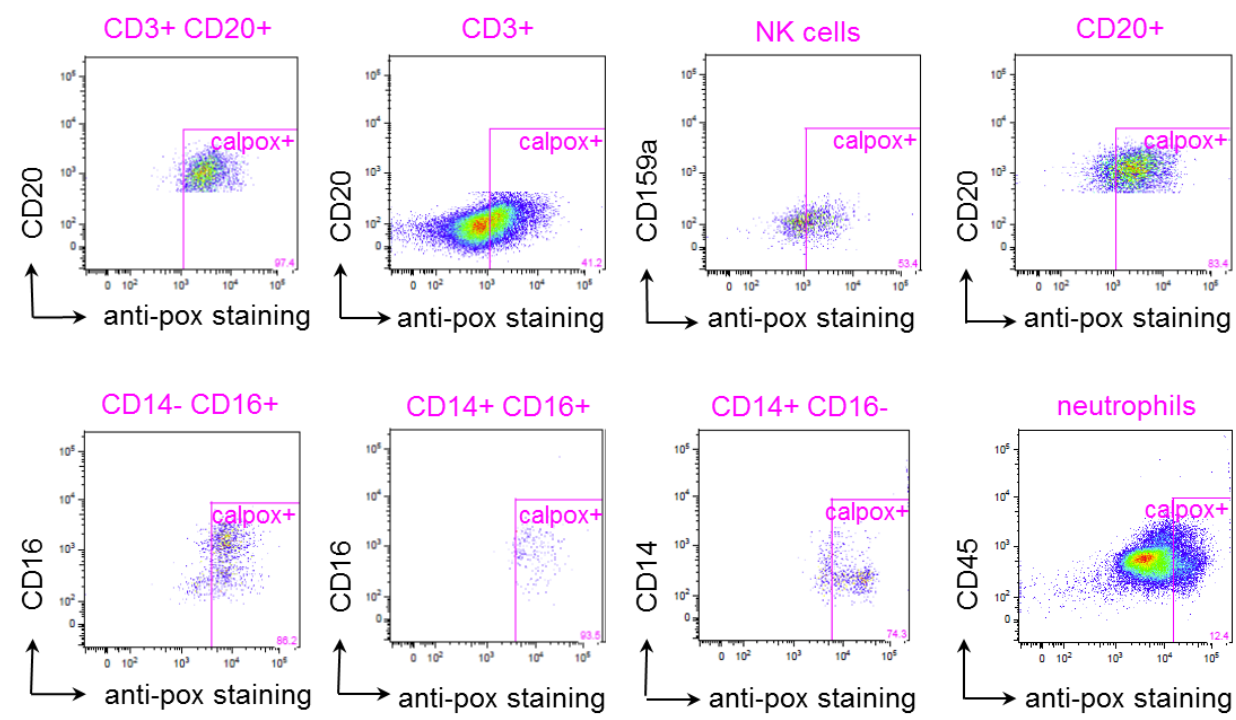

Figure 3-6. Exemplary dot plot showing calpox antigen positive $\left(\right.$ calpox $\left.^{+}\right)$immune cell populations in buffy coat. Representive dot plot of different calpox ${ }^{+}$cells are depicted. Example of VACV antigen stimulated (1:100) cells of buffy coat (\#15095) at 12 dpi. Pink lines, one gate; pink number, events within a gate given as percentages of the previous population; SSC-A, side scatter pulse area. 


\subsection{Vaccination studies - identification of immune correlates (II)}

\subsubsection{Immunization with MVTT showed highest vaccine efficacy}

The efficacy of two different live-attenuated VACV vaccines was compared after different waiting periods following immunization and by varying the vaccination route for one vaccine. One read-out for comparing vaccine efficacy was to determine the survival time of the different vaccinees after challenge with calpox virus (Figure 3-7). All mock immunized control animals died between 12 and 16 days post challenge confirming the high lethality of calpox virus in CM. By contrast, among the tested live-attenuated VACV vaccines immunization with MVTT protected more animals (67\%) than MVA (13\%), independent of the vaccination route and waiting period.

In study I that included a 4-week waiting period, $75 \%$ animals of the animals vaccinated i.n. with MVTT survived whereas all animals immunized i.m. with MVA died within 24 dpi.

In vaccination study II the waiting period was extended to 10 weeks after immunization. After this longer waiting period one MVA-vaccinated animal survived challenge virus exposure.

Independently of the route of vaccination, MVTT conferred protection between 50 and $75 \%$, which is in line with the efficacy level of MVTT following challenge after the shorter waiting period. Notably, vaccine failures survived considerably longer than the untreated control animals. The time period until the animals died (days post challenge) differed significantly between the vaccine failures and the mock immunized group (***; $\mathrm{p}=0.0002$ ).

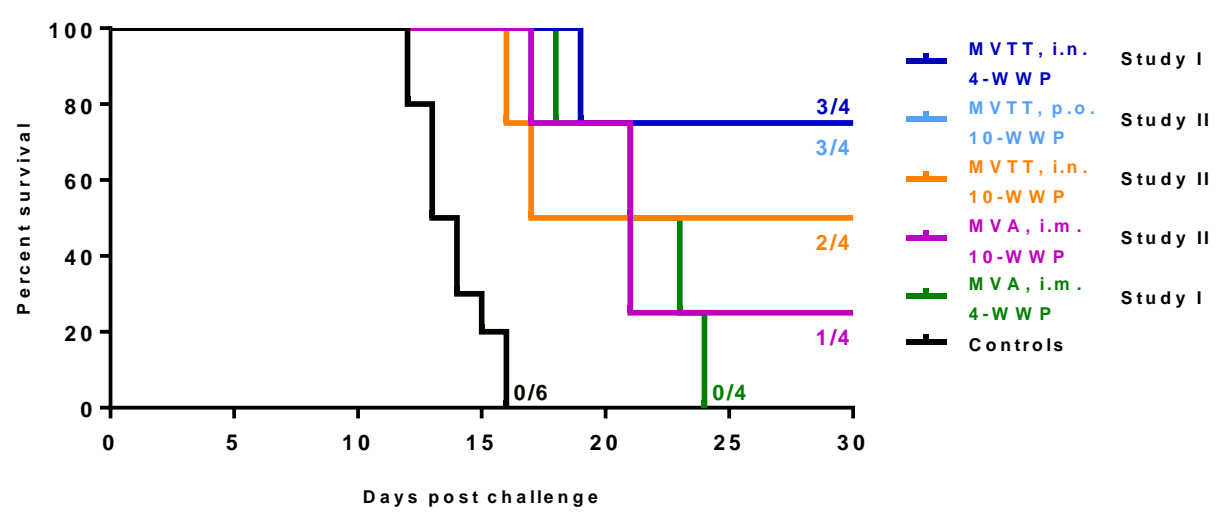

Figure 3-7. Kaplan-Meier plot on survival of vaccinated and unvaccinated $\mathrm{CM}$ after a lethal challenge with calpox virus. Comparing the survival of the different vaccinees shows that MVTT protected more individuals (67 \%) than MVA (13 $\%$ ), independently of the vaccination route. When different vaccination strategies are compared vacciniation with MVTT p.o. protected more animals than MVTT i.n. in vaccination study II (10-weeks waiting period). All mock immunized control animals died between 12 and $16 \mathrm{dpc}$. dpc, days post challenge; i.m., intramuscular immunization; i.n., intranasal immunization; MVA, modified vaccinia virus Ankara; MVTT, modified vaccinia virus Tiantan; PBS, Phosphate-buffered saline; p.o., peroral Immunization. 


\subsubsection{Post-challenge viral load confirms survival rates}

\subsubsection{Detection of calpox DNA copies in blood}

\section{Vaccination study I}

As a further read-out of the vaccination studies viral loads in blood were investigated. The second parameter besides survival to assess protection included measurement of calpox viral DNA copy number in and reisolation of infectious calpox virus from blood (see 3.3.2.2 Infectious virus detected by viral plaque assay). Calpox viral DNA was detected in blood by RTPCR in all control animals and vaccine failures. Surprisingly, all three protected vaccinees from the MVTT group exhibited transient low-level calpox DNA blips (1.9 x $10^{2}$ DNA copy numbers/ $\mu$ l blood) which appeared between days 14 and 21 and lasted in one animal until day 42 after challenge (Figure 3-8). In all infected animals viral DNA copy numbers continuously increased reaching in the controls $10^{5}-10^{7}$ copies/ $\mu 1$ blood shortly before or at death. In vaccine breakthroughs mean peak loads at death were significant lower $(\mathrm{p}=0.0159)$ compared to the controls and ranged between $7.5 \times 10^{3}-1.9 \times 10^{5}$ copies.

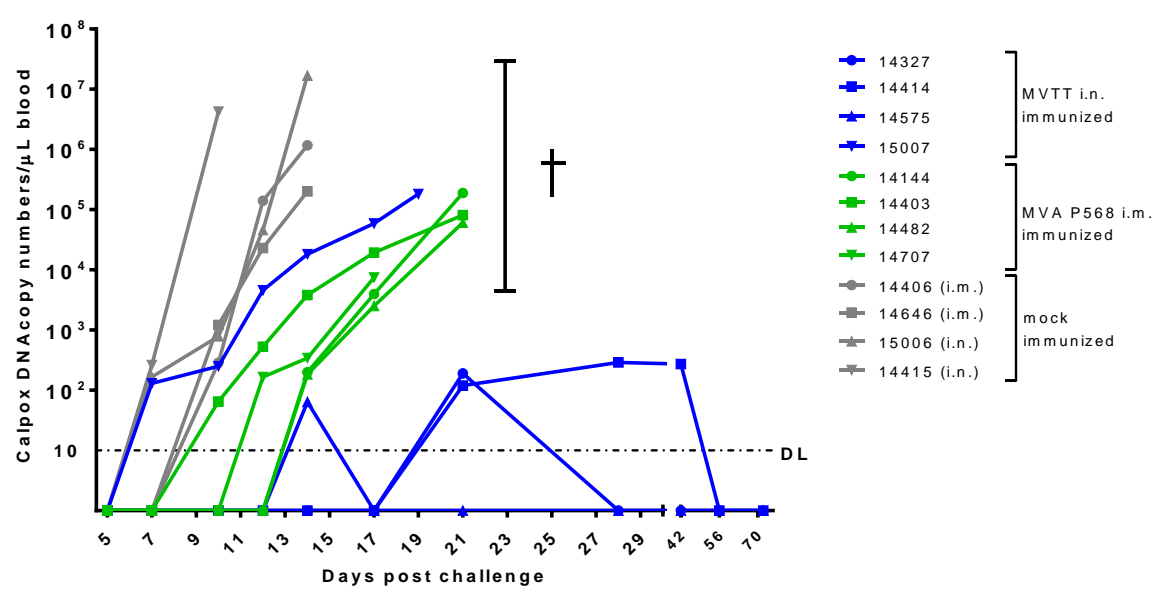

Figure 3-8. Calpox viral DNA in blood of vaccinees of study I by qRT-PCR longitudinally post challenge. Viral DNA was quantified by qRT-PCR and expressed as calpox DNA copy numbers per $\mu$ l blood. The detection limit was 10 calpox DNA copies/ $\mu$ l blood. DL, detection limit; i.m., intramuscular immunization; i.n., intranasal immunization; MVA, modified vaccinia virus Ankara; MVTT, modified vaccinia virus Tiantan; $\mathbf{P}$, passage; $\uparrow$, unprotected animals.

\section{Vaccination study II}

Calpox viral DNA loads in the second study confirmed peak levels and kinetics in control animals and unprotected vaccinees similar to what was observed in vaccination study I (Figure 3-9). While one control animal did not exceed peak viral loads above $10^{5} \mathrm{copies} / \mu 1 \mathrm{blood}$, in the other one more than $10^{10}$ copies were measured. Again, mean peak DNA viral loads in the vaccine failures were lower (but not significantly) compared to the controls $\left(3.7 \times 10^{3}-1.9 \times 10^{5}\right.$ copy numbers). Also, in two of the protected vaccines (both from either of the MVTT groups) 
transient blips of viral DNA appeared by day 14 after challenge, but dropped below the detection limit by day 21 and 29 .

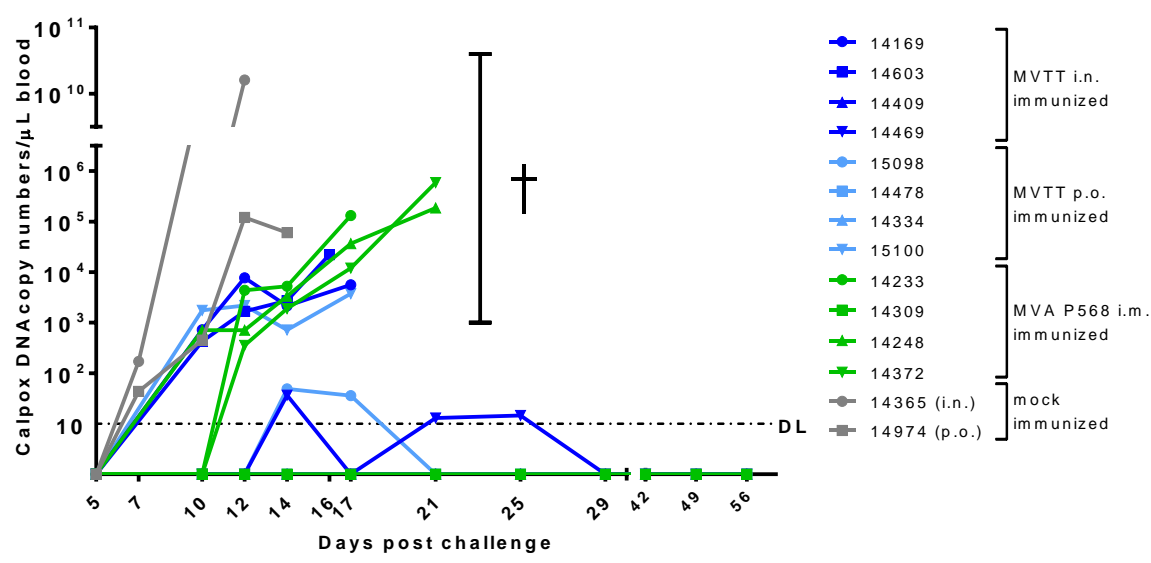

Figure 3-9. Calpox viral DNA in blood of vaccinees of study II by qRT-PCR longitudinally post challenge. Viral DNA levels were determined by qRT-PCR and are expressed as calpox DNA copy numbers per $\mu 1$ blood. The detection limit was 10 calpox DNA copies/ $\mu$ l blood. DL, detection limit; i.m., intramuscular immunization; i.n., intranasal immunization; MVA, modified vaccinia virus Ankara; MVTT, modified vaccinia virus Tiantan; P; passage; $\uparrow$, unprotected animals.

\subsubsection{Infectious virus detected by viral plaque assay}

To find out whether the transient low-level DNA viral blips in blood of protected MVTT vaccinees form study I were also mirrored by the presence of replicating calpox virus, an endpoint dilution assay was set up to isolate and quantify infectious calpox virus.

Replicating calpox virus increased strongest in all animals of the mock group (Figure 3-10). The highest value of $4.94 \times 10^{2} \mathrm{pfu} / \mu \mathrm{l}$ blood was measured in animal \#14365 at the time of death. MVA immunized vaccine failures presented with viral titres between $2 \mathrm{pfu} / \mu \mathrm{l}$ and $2.7 \times 10^{2} \mathrm{pfu} / \mu \mathrm{l}$ calpox virus at the time of death. In contrast, the two MVTT immunized vaccine failures had lower viral titres between $0.05 \mathrm{pfu} / \mu \mathrm{l}$ and $0.17 \mathrm{pfu} / \mu \mathrm{l}$ at the final stage.

No infectious virus could be isolated from any of the protected animals. 


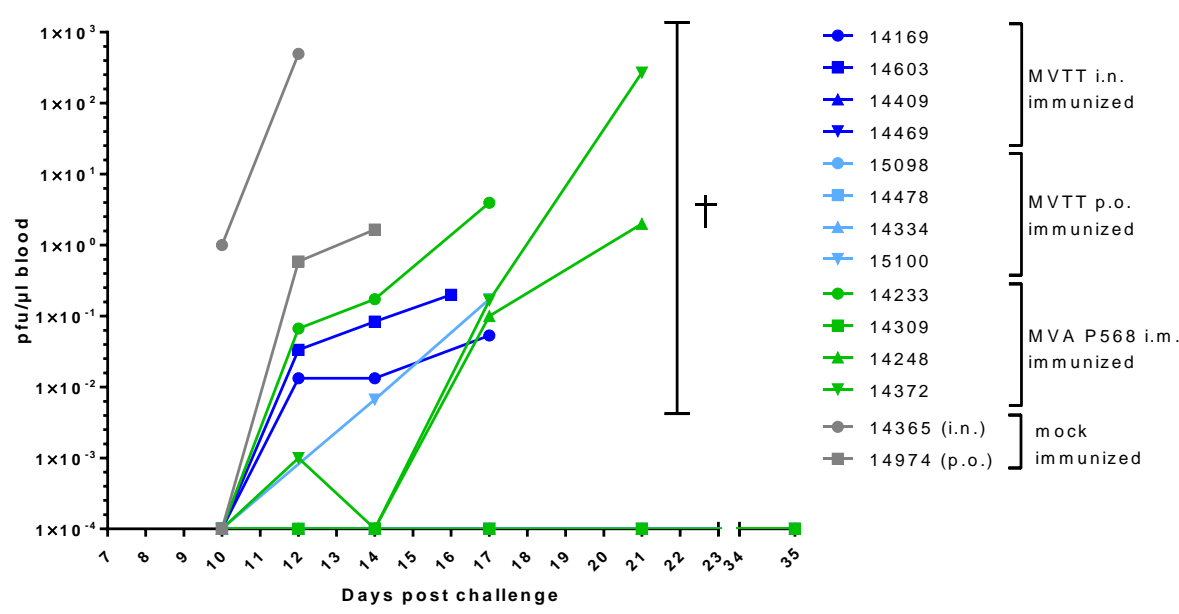

Figure 3-10. Replicating calpox virus in blood of animals from vaccine study II. Virus was isolated by cocultivation of buffy coat with Vero E6 cells. i.n., intranasal; MVA, modified vaccinia virus Ankara; MVTT, modified vaccinia virus Tiantan; p.o., per oral; $\uparrow$, unprotected animals.

\subsubsection{Humoral responses following immunization}

\subsubsection{No significant influence of binding serum Abs against whole virus parti- cles on protection}

To identify possible immune correlates between humoral immune responses and vaccine efficacy, at first binding antibodies against particles of VACV LE and calpox were analyzed by ELISA. In general, in both vaccine studies serum antibody levels against VACV LE (Figure 3-11A; Figure 3-12A) were higher than against calpox virus (Figure 3-11B; Figure 3-12B) at the time of challenge.

At challenge in vaccine study I, MVA vaccinees had considerably higher antibody levels against the VACV antigen (Figure 3-13A), while against calpox virus antigen responses were mixed and varied within the two vaccine groups (Figure 3-13B).

Significant differences in binding antibodies against VACV between vaccination groups (MVTT, i.n. and MVA, i.m.) ( $\mathrm{p}=0.0286)$ were detected for vaccination study I at day of challenge (Figure 3-14). In vaccination study II involving the 10-week waiting period, antibody levels in the MVTT vaccinees against VACV antigen were similar to or even higher than those in the MVA vaccinated animals, but in each group high variation was observed. Also, the different vaccination routes chosen for MVTT had no influence on immunogenicity, as overall levels in the MVTT vaccinees were similar.

Overall, at challenge the binding antibody level against VACV in MVA, i.m. vaccinees was higher than in the MVTT, i.n. group in study I. No other association between binding antibody levels, neither against VACV nor calpox antigen, and protection were noticed in any of the vaccinees. 
Two weeks after challenge in all animals from vaccination study I immunized with MVA, antibody levels against both OPXV antigens markedly increased suggesting an anamnestic response (Figure 3-11). The same was true for three of the four animals vaccinated with MVTT. Interestingly, this rise was not only observed in the one vaccine failure, but also in two vaccinees which according to the criteria survival and viral load were considered to be protected. The majority of animals from vaccination study II independant of being protected or not protectd also developed anamnestic antibody responses against both viral antigens except for two protected animals, i.e. one MVA vaccinee (\# 14309) and one MVTT vaccinee (\#14334) (Figure 3-12).

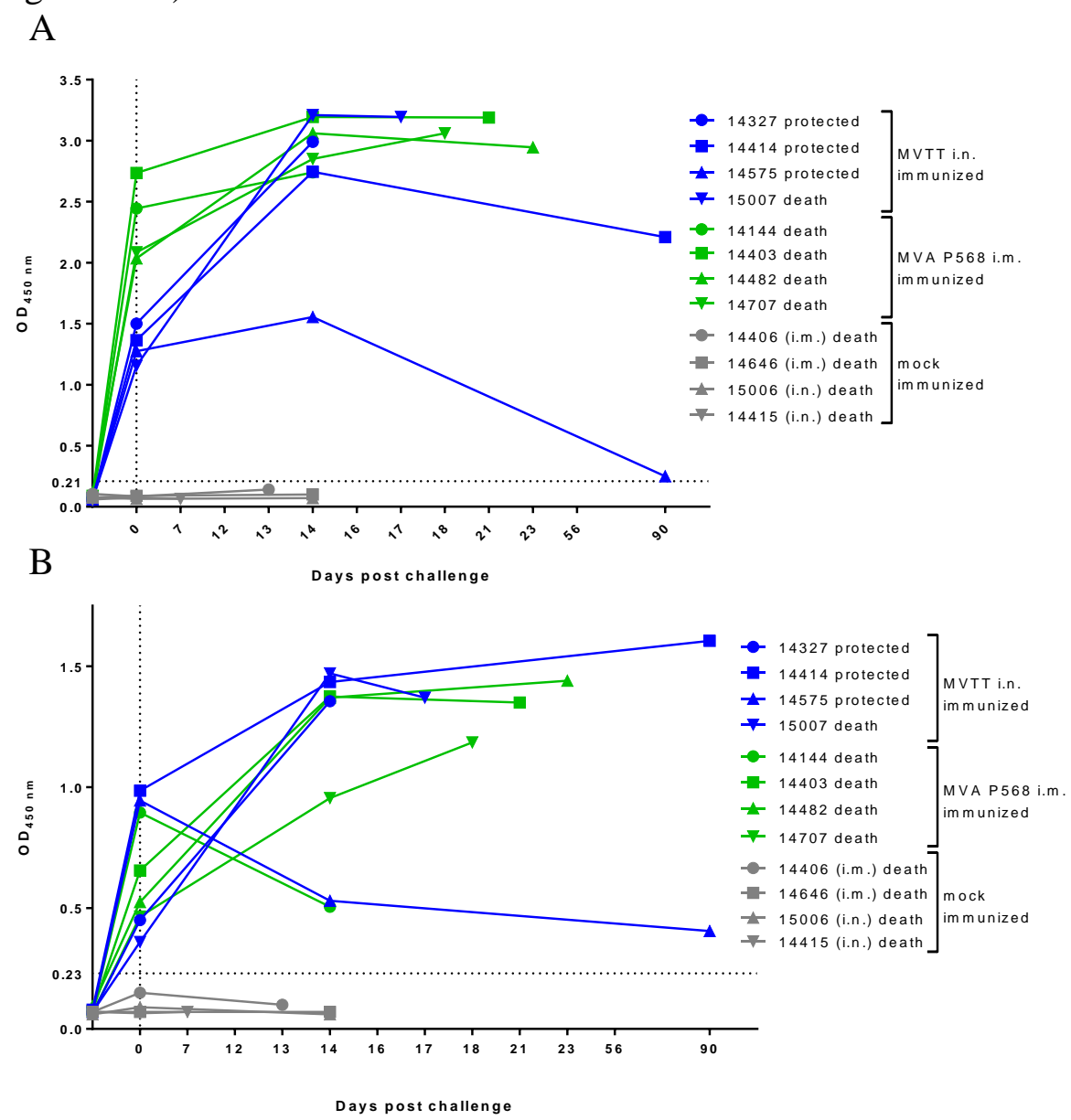

Figure 3-11. Levels of vaccine-induced binding antibodies were not associated with protection in vaccination study $I$. Binding antibodies against (A) VACV LE and (B) calpox virus were determined by ELISA in plasma from the day of challenge and after challenge. The cut-off (0.21 and 0.23$)$ was double the value of the highest OD of each of the negative controls. Plasma samples were were diluted 1:200 in blocking buffer and $100 \mu 1 /$ well was used in double detection. i.m., intramuscular Immunization; i.n., intranasal Immunization; MVA, modified vaccinia virus Ankara; MVTT, modified vaccinia virus Tiantan; p.o., peroral immunization; $\mathbf{P}$, passage.

All mock immunized control animals remained antibody negative. 

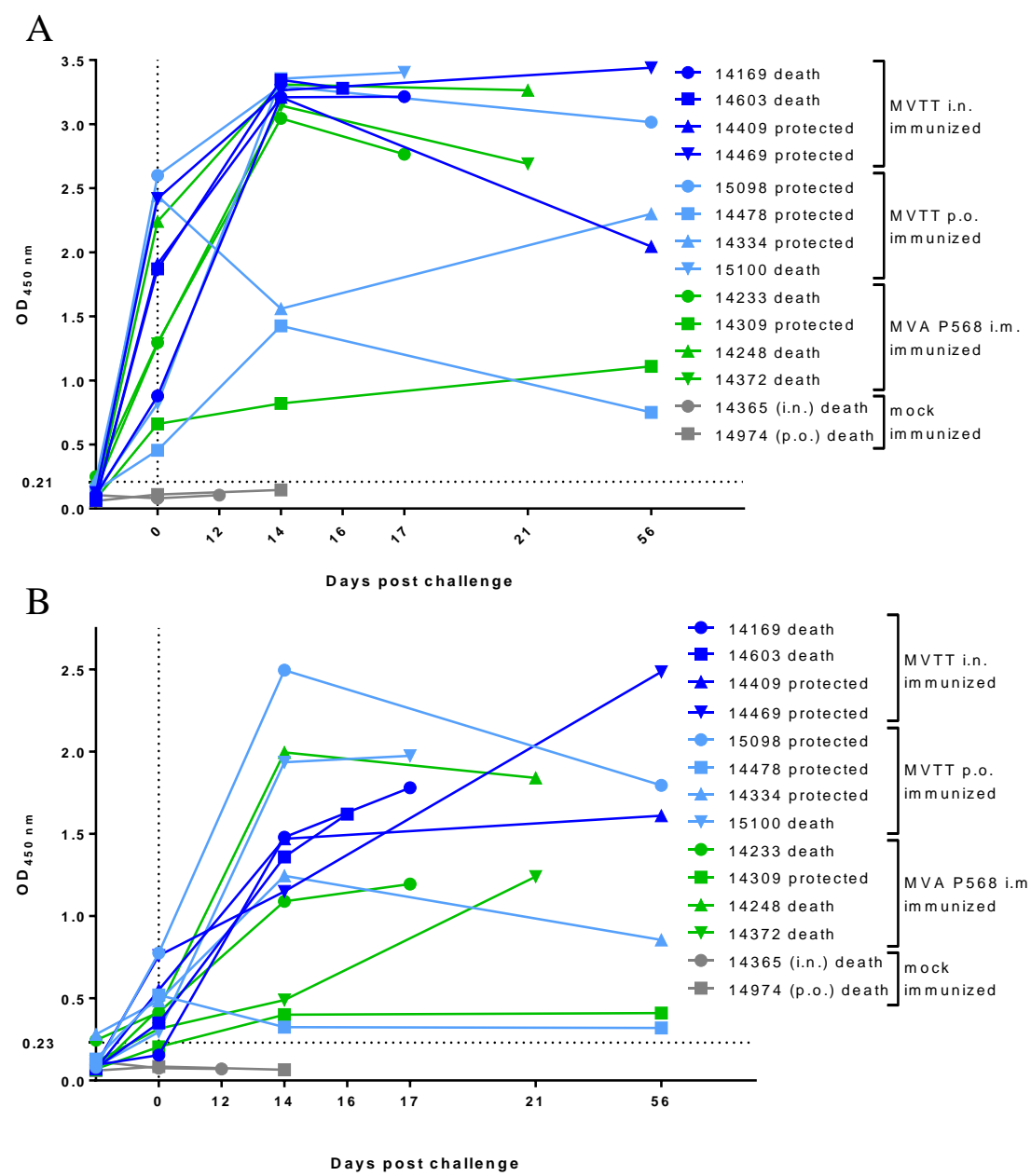

Figure 3-12. Levels of vaccine-induced binding antibodies were not associated with protection in vaccination study II. Binding antibodies against (A) VACV LE and (B) calpox virus were quantified by ELISA. The cut-off value (0.21 and 0.23) was the double amount of the highest OD value of each of the negative controls. Plasma samples were diluted 1:200 in blocking buffer and $100 \mu \mathrm{l} /$ well was used in dublicate. i.m., intramuscular immunization; i.n., intranasal immunization; MVA, modified vaccinia virus Ankara; MVTT, modified vaccinia virus Tiantan; p.o., peroral immunization; P, passage.

A

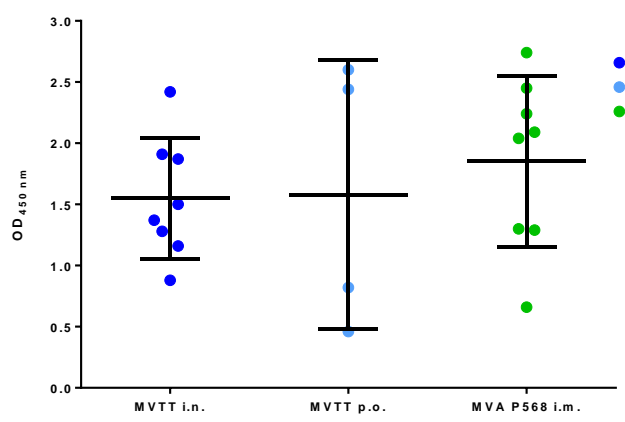

B

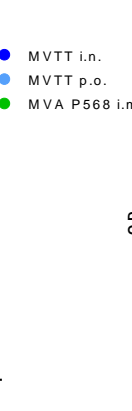

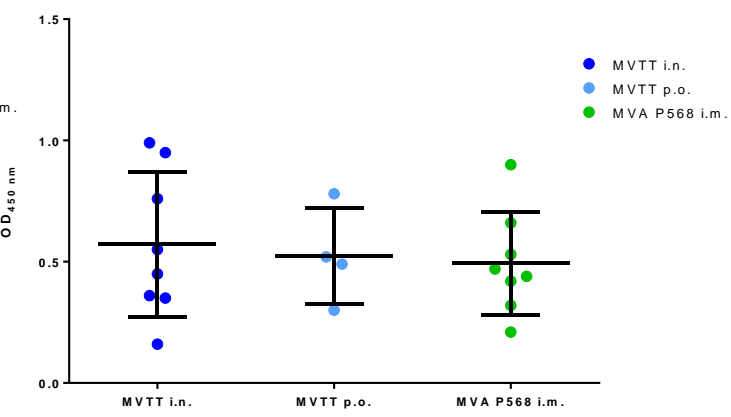

Figure 3-13. Results for vaccination study I and II revealed no differences in binding antibodies between vaccination groups by ELISA at day of challenge. Binding antibodies against (A) VACV LE and (B) calpox virus were determined by ELISA. No significant differences were detected when vaccination groups in study I and II were compared together. However, significant differences in binding antibodies against VACV between groups (MVTT, i.n. and MVA, i.m.) $(\mathrm{p}=0.0286)$ were detected for vaccination study I at day of challenge (see Figure 3-14). Mean \pm SD of all animals per group are shown. i.m., intramuscular immunization; i.n., intranasal immunization; MVA, modified vaccinia virus Ankara; MVTT, modified vaccinia virus Tiantan; p.o., peroral immunization; $\mathbf{P}$, passage. 


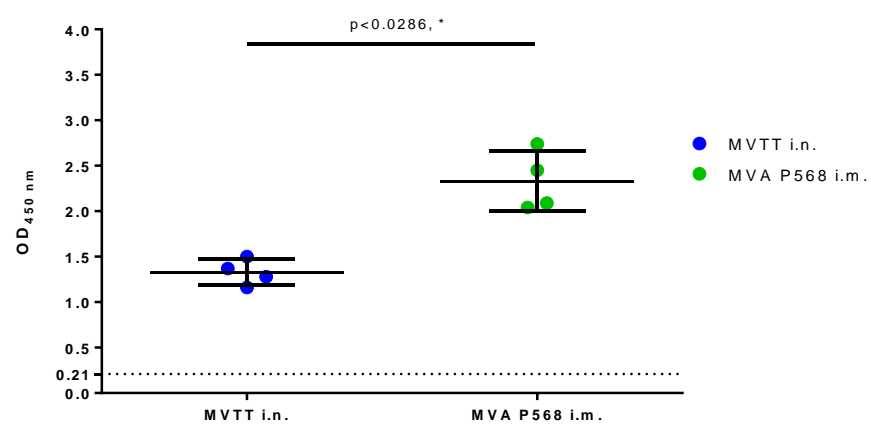

Figure 3-14. Results for vaccination study I revealed significant differences in binding antibodies determined by ELISA between vaccination groups at challenge. Coating with $5 \mu \mathrm{g} / \mathrm{ml}$ VACV. Mean \pm SD of all animals per group is shown. The mean value of the MVA immunized group was significantly higher than that of the MVTT immunized group at challenge. The cut-off value $(0.21$ and 0.23$)$ was the double amount of the highest OD value of each of the negative controls. i.m., intramuscular immunization; i.n., intranasal immunization; MVA, modified vaccinia virus Ankara; MVTT, modified vaccinia virus Tiantan; $\mathbf{P}$, passage; $\mathbf{p}$, significance level.

The antibody levels against calpox virus of all protected animals were higher than those of the unprotected animals of vaccination study I and II (Figure 3-15A). However, differences did not reach significance.
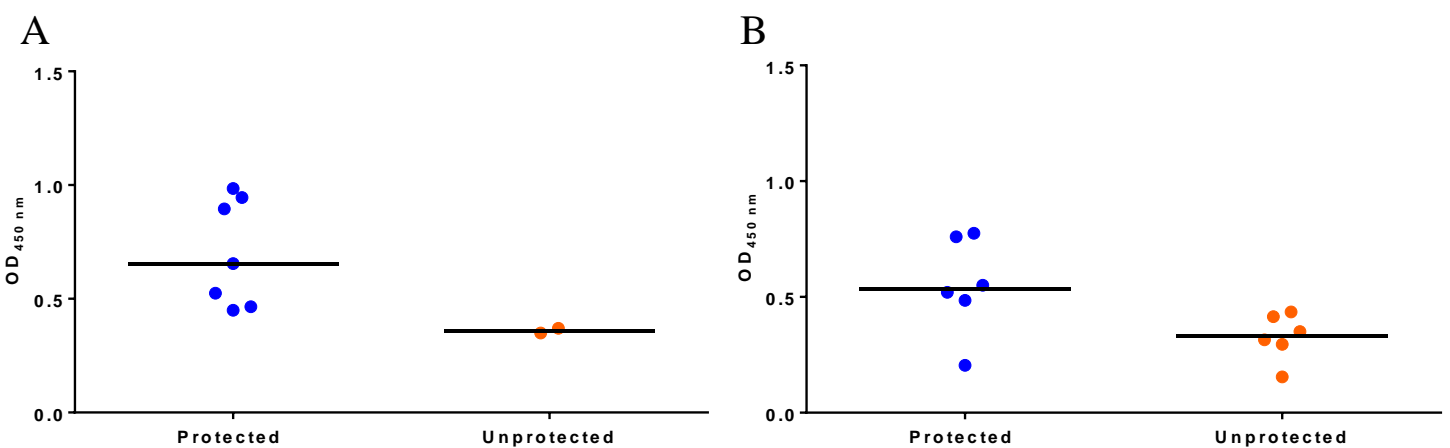

Figure 3-15. ELISA antibodies against viral particles of calpox virus at the day of challenge virus exposure. Medians of all protected animals against unprotected animals of vaccination study I (A) and vaccination study II (B) are shown. Statistics revealed no significance.

\subsubsection{Successful validation of the poxvirus peptide microarray chip}

MAbs against already known epitope regions were used to validate the poxvirus peptide microarray chip. All regions of the epitopes were mapped identically to the known epitope regions by peptide microarrays (data not shown).

\subsubsection{High resolution mapping of antibody linear targets revealed unique epitopes in protected $\mathrm{CM}$}

ELISA test applying whole viral particles cannot discriminate between the quality of antibody responses in protected and unprotected CM. Therefore, an OPXV-specific high-resolution peptide microarray assay was employed to map antibodies against calpox virus linear 
epitopes, which can be correlated with protection from infection. Plasma samples from control animals as well as all vaccinees before and after challenge were screened with peptide microarray chips. In vaccinees before challenge, no reaction with unique epitopes can be correlated to protection (see Appendix: Table A.3-3 - A.3-14). Surprisingly, four epitopes from three of the six investigated proteins were exclusively observed in plasma collected post challenge from protected animals (Table 3-1).

Table 3-1: List of detected epitopes in protected animals after challenge.

\begin{tabular}{l|l|lllll}
\hline $\begin{array}{l}\text { Epitope } \\
\text { no. }\end{array}$ & Study & Vaccine & Protein & $\begin{array}{l}\text { Animal } \\
\text { ID }\end{array}$ & $\begin{array}{l}\text { Spot } \\
\text { no. }\end{array}$ & Sequence \\
\hline 1 & I & MVTT, i.n. & L1 & 14327 & 245 & 28-ASAQTKCDIEIGNFY-42 \\
2 & I & MVTT, i.n. & A33 & 14414 & $337-338$ & 64-SANEAAITDAAVAVA-81 ${ }^{1}$ \\
3 & II & MVTT, i.n. & L1 & 14469 & 240 & 79-LTPEQKAYVPAMFTA-93 ${ }^{1}$ \\
& & MVTT, p.o. & & 15098 & 240 & \\
4 & II & MVTT, i.n. & A33 & 14469 & $370-371$ & 163-TSDYQDSDVSQEVRKYFC-180 ${ }^{1}$ \\
& & MVTT, p.o. & & 15098 & $370-371$ & \\
5 & II & MVTT, i.n. & B5 & 14469 & $452-455$ & 235-PICVRTNEEFDPVDDGPDDETDLS-258 \\
& & MVTT, p.o. & & 15098 & $452-455$ & \\
\hline
\end{tabular}

ID, identification; i.n., intranasal Immunization; MVTT, modified vaccinia virus Tiantan; no., number; p.o., peroral Immunization; Study, Vaccination study.

${ }^{1}$ The epitopes 1-4 were only detected after challenge.

${ }^{2}$ Epitope 5 was already detected at 2 weeks post second immunization.

In study II the total number of epitopes (12 epitopes) identifies during immunization was higher than in study I (4 epitopes) (Table 3-2).

Table 3-2: List of all mapped epitopes induced by vaccination.

\begin{tabular}{|c|c|c|c|c|c|c|}
\hline Study & $\begin{array}{l}\text { Epitope } \\
\text { no. }\end{array}$ & Vaccine & Protein & $\begin{array}{l}\text { Animal } \\
\text { ID }\end{array}$ & $\begin{array}{l}\text { Spot } \\
\text { no. }\end{array}$ & Sequence \\
\hline \multirow[t]{11}{*}{$\mathrm{I}$} & 1 & MVA, i.m. & B5 & 14482 & 458 & 253-DETDLSKLSKDVVQY-267 \\
\hline & 2 & MVTT, i.n. & D8 & 14414 & 97 & 190-NIHSDQLSKFRTLLS-204 \\
\hline & & MVTT, i.n. & D8 & 14327 & 97 & 190-NIHSDQLSKFRTLLS-204 \\
\hline & 3 & MVTT, i.n. & B5 & 14327 & $381-383$ & 22-TVPTMNNAKLTSTETSFNDKQ-42 \\
\hline & 4 & MVA, i.m. & B5 & 14144 & $398-400$ & 73-KKMCTVSDYISELYNKPLYEV-93 \\
\hline & & MVA, i.m. & B5 & 14403 & $398-400$ & 73-KKMCTVSDYISELYNKPLYEV-93 \\
\hline & & MVA, i.m. & B5 & 14482 & $398-400$ & 73-KKMCTVSDYISELYNKPLYEV-93 \\
\hline & & MVA, i.m. & B5 & 14707 & $398-400$ & 73-KKMCTVSDYISELYNKPLYEV-93 \\
\hline & & MVTT, i.n. & B5 & 14414 & $398-400$ & 73-KKMCTVSDYISELYNKPLYEV-93 \\
\hline & & MVTT, i.n. & B5 & 14575 & $398-400$ & 73-KKMCTVSDYISELYNKPLYEV-93 \\
\hline & & MVTT, i.n. & B5 & 15007 & $398-400$ & 73-KKMCTVSDYISELYNKPLYEV-93 \\
\hline \multirow[t]{6}{*}{ II } & 1 & MVA, i.m. & A27 & 14309 & 3 & 7-PGDDDLAIPATEFFS-21 \\
\hline & 2 & MVA, i.m. & A27 & 14233 & 12 & 34-AIVKADEDDNEETLK-48 \\
\hline & 3 & MVTT, p.o. & D8 & 15098 & 34 & 1-MPQQLSPINIETKKA-15 \\
\hline & 4 & MVA, i.m. & D8 & 14233 & 47 & 40-GKLVRINFKGGYISG-54 \\
\hline & & MVTT, p.o. & D8 & 15098 & 47 & 40-GKLVRINFKGGYISG-54 \\
\hline & 5 & MVTT, p.o. & D8 & 15098 & $79-80$ & 136-NQLDSIRSANTSAPFDSV-153 \\
\hline
\end{tabular}




\begin{tabular}{|c|c|c|c|c|c|}
\hline 6 & MVA, i.m. & D8 & 14233 & 81 & 142-RSANTSAPFDSVFYL-156 \\
\hline 7 & MVA, i.m. & D8 & 14372 & $102-103$ & 205-SSNHDGKPHYITENYRNP-222 \\
\hline \multirow[t]{3}{*}{8} & MVTT, i.n. & A33 & 14469 & $317-319$ & 4-PENDEEQTSVFSATVYGDKIQ-24 \\
\hline & MVTT, i.n. & A33 & 14409 & $317-319$ & 4-PENDEEQTSVFSATVYGDKIQ-24 \\
\hline & MVTT, p.o. & A33 & 15098 & $317-319$ & 4-PENDEEQTSVFSATVYGDKIQ-24 \\
\hline 9 & MVTT, p.o. & A33 & 15098 & 370 & 163-TSDYQDSDVSQEVRK-177 \\
\hline \multirow[t]{2}{*}{10} & MVTT, i.n. & B5 & 14469 & $454-455$ & 244-FDPVDDGPDDETDLSKLS-261 \\
\hline & MVTT, p.o. & B5 & 15098 & $452-455$ & 235-PICVRTNEEFDPVDDGPDDETDLS-258 \\
\hline \multirow[t]{6}{*}{11} & MVA, i.m. & B5 & 14309 & $457-478$ & 250-GPDDETDLSKLSKDVVQY-267 \\
\hline & MVA, i.m. & B5 & 14248 & $457-478$ & 250-GPDDETDLSKLSKDVVQY-267 \\
\hline & MVA, i.m. & B5 & 14372 & $457-478$ & 250-GPDDETDLSKLSKDVVQY-267 \\
\hline & MVTT, i.n. & B5 & 14603 & $457-478$ & 250-GPDDETDLSKLSKDVVQY-267 \\
\hline & MVTT, p.o. & B5 & 14334 & $457-478$ & 250-GPDDETDLSKLSKDVVQY-267 \\
\hline & MVTT, p.o. & B5 & 15100 & $457-478$ & 250-GPDDETDLSKLSKDVVQY-267 \\
\hline \multirow[t]{9}{*}{12} & MVA, i.m. & B5 & 14233 & $398-400$ & 73-KKMCTVSDYISELYNKPLYEV-93 \\
\hline & MVA, i.m. & B5 & 14309 & $398-400$ & 73-KKMCTVSDYISELYNKPLYEV-93 \\
\hline & MVA, i.m. & B5 & 14248 & $398-400$ & 73-KKMCTVSDYISELYNKPLYEV-93 \\
\hline & MVA, i.m. & B5 & 14372 & $398-400$ & 73-KKMCTVSDYISELYNKPLYEV-93 \\
\hline & MVTT, i.n. & B5 & 14469 & $399-400$ & 73-KKMCTVSDYISELYNKPLYEV-93 \\
\hline & MVTT, i.n. & B5 & 14603 & $398-400$ & 73-KKMCTVSDYISELYNKPLYEV-93 \\
\hline & MVTT, p.o. & B5 & 15098 & $398-400$ & 73-KKMCTVSDYISELYNKPLYEV-93 \\
\hline & MVTT, p.o. & B5 & 14334 & $399-400$ & 73-KKMCTVSDYISELYNKPLYEV-93 \\
\hline & MVTT, p.o. & B5 & 15100 & $398-400$ & 73-KKMCTVSDYISELYNKPLYEV-93 \\
\hline
\end{tabular}

ID, identification; i.m., intramuscular immunization; i.n., intranasal immunization; MVA, modified vaccinia virus Ankara; MVTT, modified vaccinia virus Tiantan; no., number; p.o., peroral immunization; Study, vaccination study.

\subsubsection{Neutralizing antibodies did not correlate with protection}

Neutralizing antibodies against VACV LE and calpox virus were determined by a plaque reduction neutralization test (PRNT) in plasma collected at day of challenge (Figure 3-16). In vaccination study I the titres against VACV LE varied between 1:20 and 1:320 in all immunized animals. No differences between the two vaccine groups were evident. When samples were tested against calpox virus the titres varied between 1:10 and 1:80 in all immunized animals indicating lower neutralizing capacity against the challenge virus compared to VACV. Two of the vaccinees (one protected MVTT immunized animal, \#14414, and one unprotected MVA immunized animal, \#14403) were tested negative for neutralizing antibodies against calpox virus. Neutralizing titres were not associated with protection. Being in line with this, neutralizing antibodies were also detected in animals that died, e.g. \#15007. 


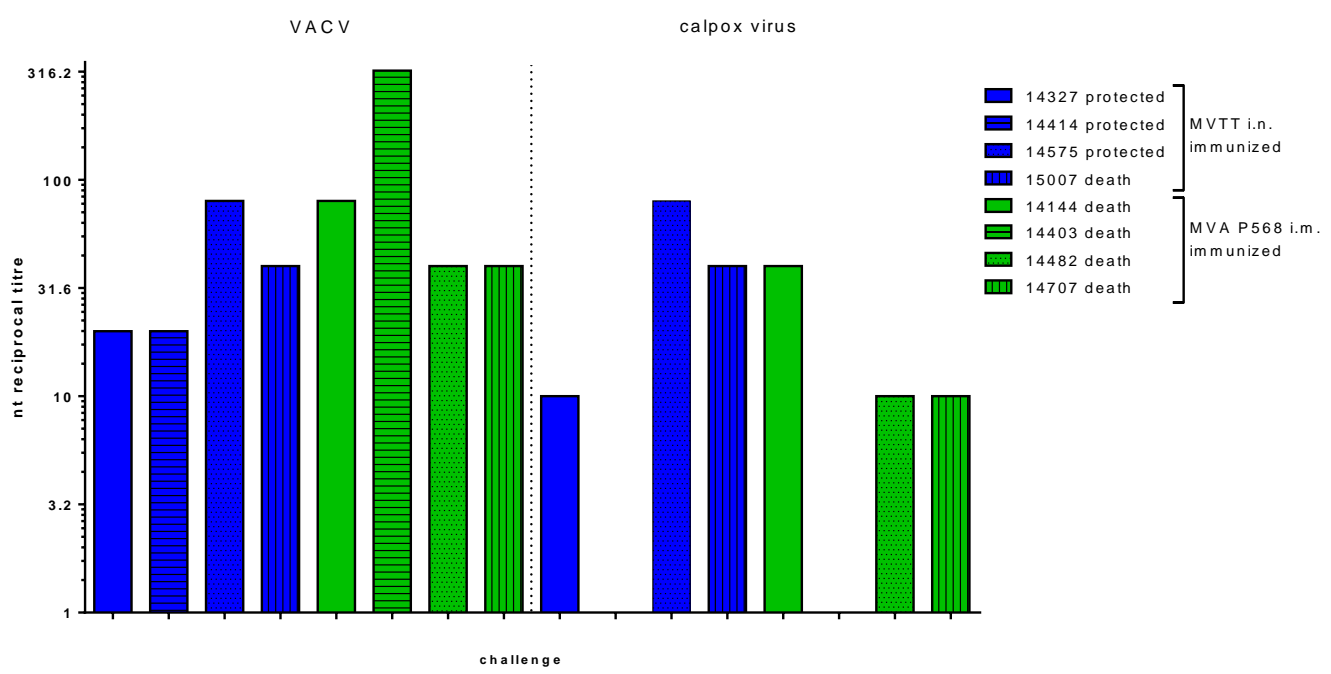

Figure 3-16. Neutralizing antibodies against VACV LE and calpox virus in animals of vaccination study I at time of challenge. Values are shown as mean of duplicate testing. Plasma from control animals was always negative. i.m., intramuscular immunization; i.n., intranasal immunization; MVA, modified vaccinia virus Ankara; MVTT, modified vaccinia virus Tiantan; nt, neutralizing antibody titre as determined by PRNT; P, passage.

The mean values against calpox virus were lower than against VACV LE (Figure 3-17). No significant difference between the mean values of the titres between the groups was detected.

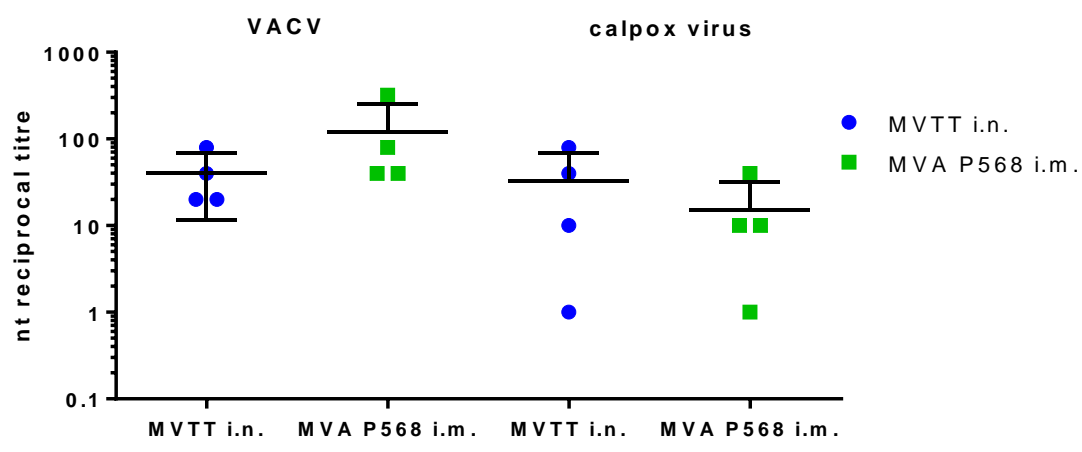

Figure 3-17. Titres of neutralizing antibodies per vaccination group in vaccination study I. Plasma samples were examined pre-immunization and at the day of challengefor the presence of VACV LE and calpox virus neutralizing antibodies by a plaque reduction test. The vaccine groups did not differ significantly from each other. Controls were alsways negative. Mean with SD is shown. i.m., intramuscular immunization; i.n., intranasal immunization; MVA, modified vaccinia virus Ankara; MVTT, modified vaccinia virus Tiantan; nt, neutralizing antibody titre as determined by PRNT; $\mathbf{P}$, passage.

In vaccinees from vaccination study II neutralizing antibody titres against VACV LE were lower compared to vaccination study I ranging between 1:10 and 1:80 (Figure 3-18). The neutralizing activity against calpox virus was in the same range (Figure 3-19B). Notably, the mean neutralizing titre against VACV in the group vaccinated perorally with MVTT was significantly higher compared to the MVA vaccinated animals (Figure 3-19, $\mathrm{p}=0.0487$ ).

In summary, there were no significant differences between neutralizing titres in protected and unprotected animals in study II. 


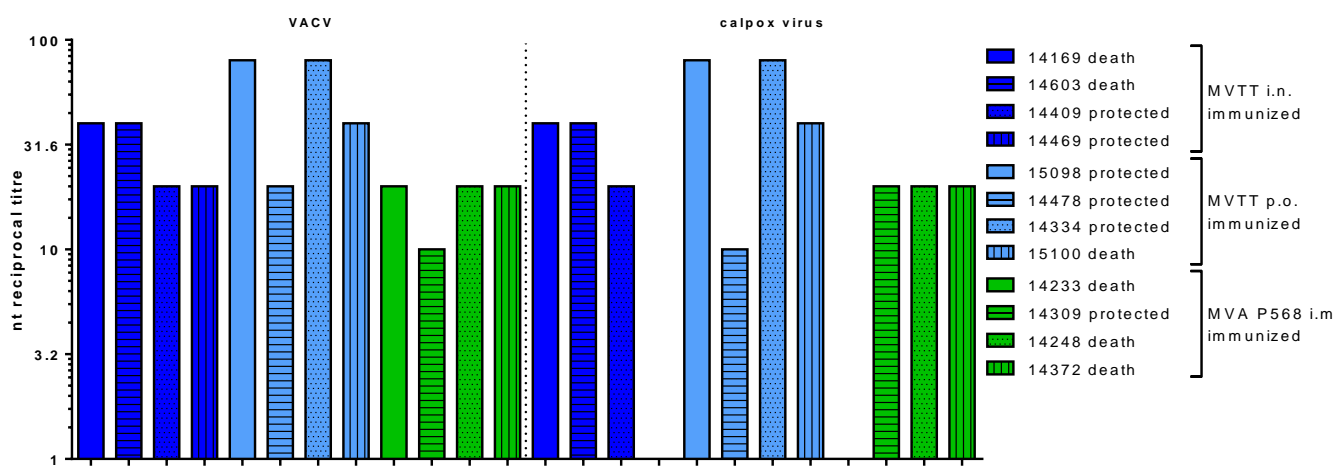

challenge

Figure 3-18. Neutralizing antibodiy titres in animals of vaccination study II. Plasma samples were examined at the day of challenge for the presence of VACV LE and calpox virus neutralizing antibodies by a plaque reduction test. The vaccine groups did not differ significantly from each other. Controls were always negative. i.m., intramuscular immunization; i.n., intranasal immunization; MVA, modified vaccinia virus Ankara; MVTT, modified vaccinia virus Tiantan; nt, neutralizing antibody titre as determined by PRNT; p.o., peroral immunization; $\mathbf{P}$, passage

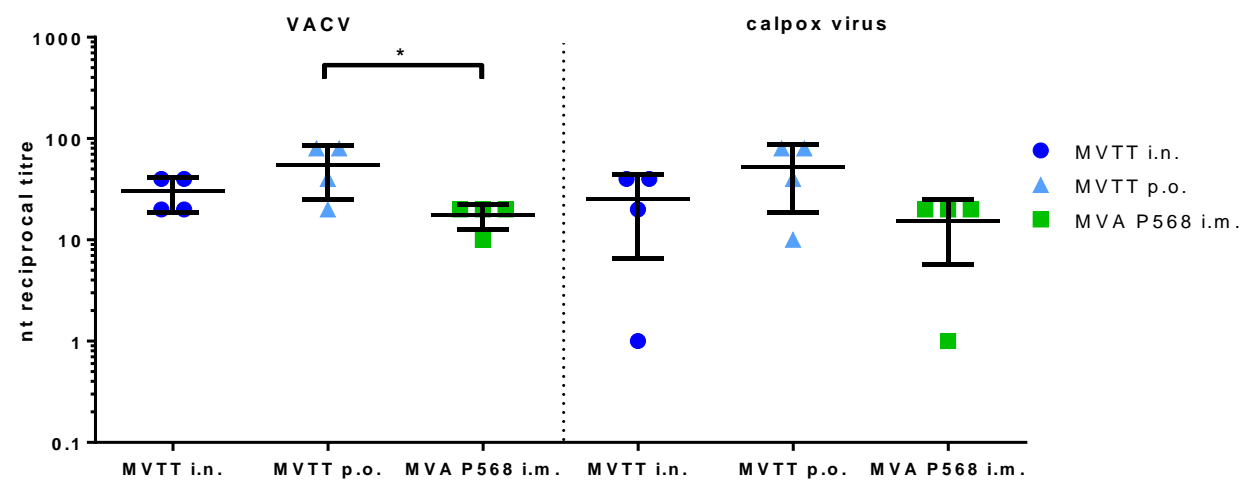

Figure 3-19. Mean \pm SD of the detected titres of neutralizing antibodies per vaccination group for vaccination study II. Plasma samples collected at the day of challenge were examined for the presence neutralizing antibodies against VACV LE and calpox virus. The titres against VACV LE of the MVTT p.o. immunized group were significantly higher $(p=0.0487)$ than those of the MVA i.m. immunized group titre .m., intramuscular immunization; i.n., intranasal immunization; MVA, modified vaccinia virus Ankara; MVTT, modified vaccinia virus Tiantan; nt, neutralizing antibody titre as determined by PRNT; P, passage; *, p < 0.05 .

The analyses showed that the levels of neutralizing antibodies were higher after the 4-week waiting period, but there was no significant difference notable (i).

Compared to the neutralizing antibody titres after the 4-week waiting period, the titres were lower after the 10-week waiting period (ii).

No association was detectable between the development of levels of neutralizing antibodies and protection (iii). Animals mit moderate neutralizing antibody titres were not protected and animals without were protected as well. 


\subsubsection{Phenotyping of immune cells and cellular immune response}

\subsubsection{Longitudinal phenotyping of innate and adaptive immune cells in whole blood}

The phenotyping of different immune cells was done to assess the influence of VACV immunization and of calpox virus infection on a variety of cell subsets of the innate and adaptive immune response. A particular focus was laid on monocytes/macrophages because this subset is discussed to be responsible for spreading the virus (Davies and Parekh, 2017). Moreover, the proliferative cellular response against VACV was investigated and related to challenge outcome. For these kind of analyses only material from vaccination study II was available.

\section{Longitudinal phenotyping of innate immune cells}

In $\mathrm{CD}_{14}{ }^{+}$cells (monocytes) (Figure 3-20) and myeloid dendritic cells (mDCs) (Figure 3-21) was a cell drop detected after immunization. The cell numbers increased at $21 \mathrm{dpc}$. Cell numbers increased in moribund animals (\#14248 and \#14372).

In pDCs (Figure 3-22), NK cells (Figure 3-23) and $\gamma \delta$ T cells (Figure 3-24) no differences were observed in absolute cell numbers during the analyzed time.

\section{Longitudinal analysis of CD14 ${ }^{+}$cells (monocytes) and myeloid dendritic cells (mDCs)}

Absolute numbers of $\mathrm{CD}_{1} 4^{+}$monocytes (Figure 3-20) and myeloid dendritic cells (mDCs) (Figure 3-21) dropped after immunization and increased after challenge at day 21. Notably, cell numbers increased further in two moribund animals that were vaccinated with MVA (\#14248 and \#14372).

No significant differences were detected in absolute cell numbers between vaccination groups at the day of challenge. There was also no significant difference between cell numbers of protected and unprotected animals at challenge. 


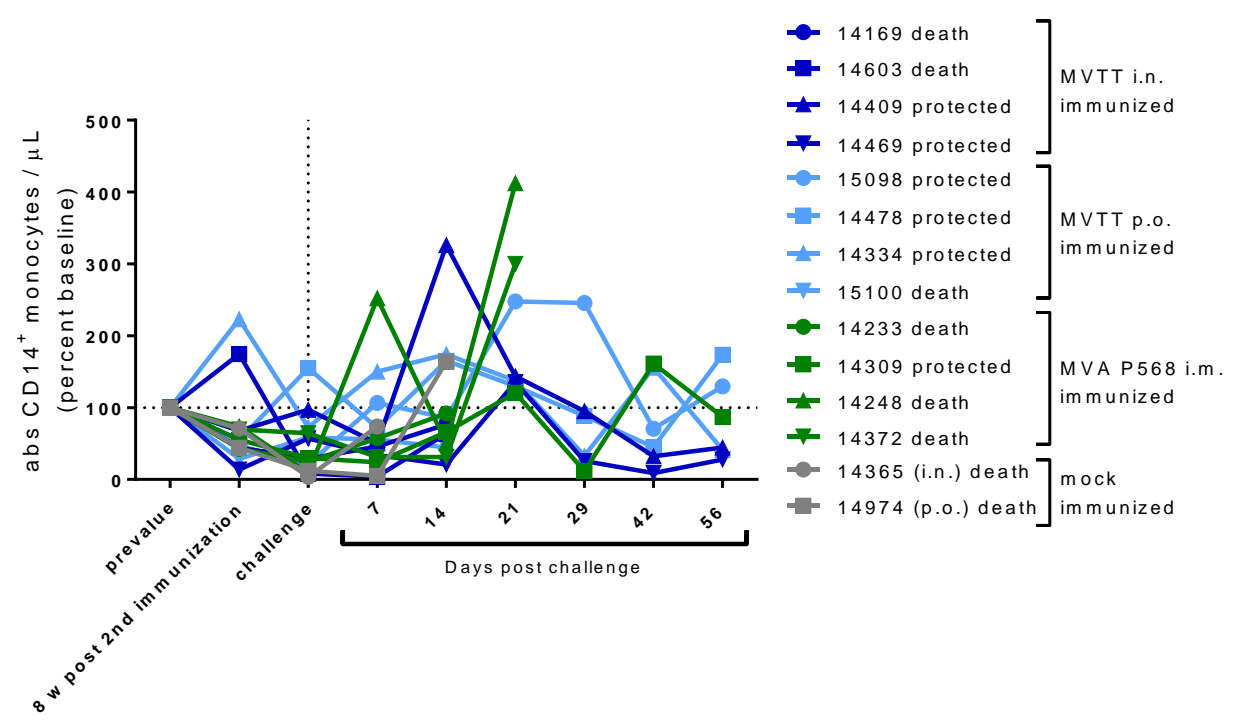

Figure 3-20. Absolute cell numbers of $\mathrm{CD14}^{+}$cells (monocytes) in vaccination study II are shown over time. Absolute cell numbers are given in percent baseline. abs, absolute cell numbers; dpc, days post challenge; i.n., intranasal; i.m., intramuscular; MVA, modified vaccinia virus Ankara; MVTT, modified vaccinia virus Tiantan; p.o., per oral; P, passage, w, weeks.
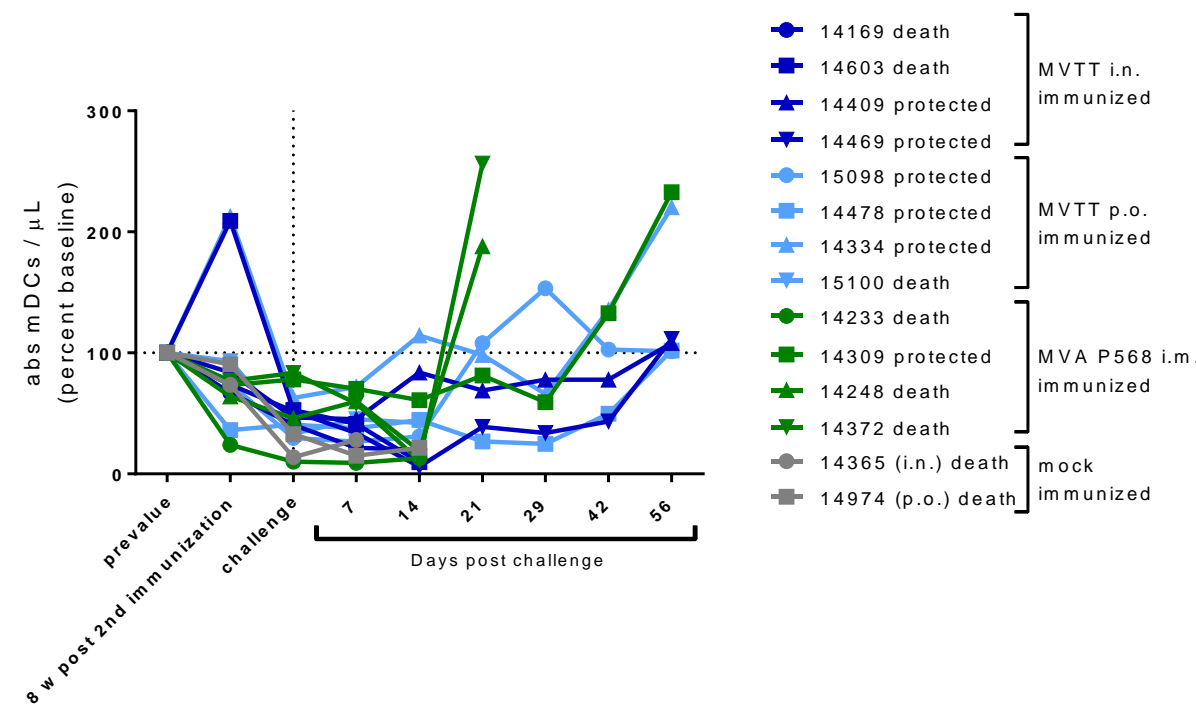

Figure 3-21. Absolute cell numbers of myeloid dendritic cells (mDCs) in vaccination study II are shown over time. Absolute cell numbers are given in percent baseline. abs, absolute cell numbers; dpc, days post challenge; i.n., intranasal; i.m., intramuscular; MVA, modified vaccinia virus Ankara; MVTT, modified vaccinia virus Tiantan; p.o., per oral; P, passage, w, weeks.

\section{Longitudinal analysis of pDCs, NK cell and $\gamma \delta \mathrm{T}$ cell numbers}

In the majority of animals, no significant changes in the absolute cell numbers of pDCs (Figure 3-22), NK cells (Figure 3-23), and $\gamma \delta \mathrm{T}$ cells (Figure 3-24) were observed. Notably, cell numbers increased in two moribund animals that were vaccinated with MVA (\#14248 and \#14372). Furthermore, one protected MVTT p.o. animal (\#14334) had higher absolute $\gamma \delta \mathrm{T}$ 
cell numbers compared to the other animals. They increased 900 -fold at 8 -weeks post second immunization, decreased 600-fold at challenge, but remained higher after challenge.

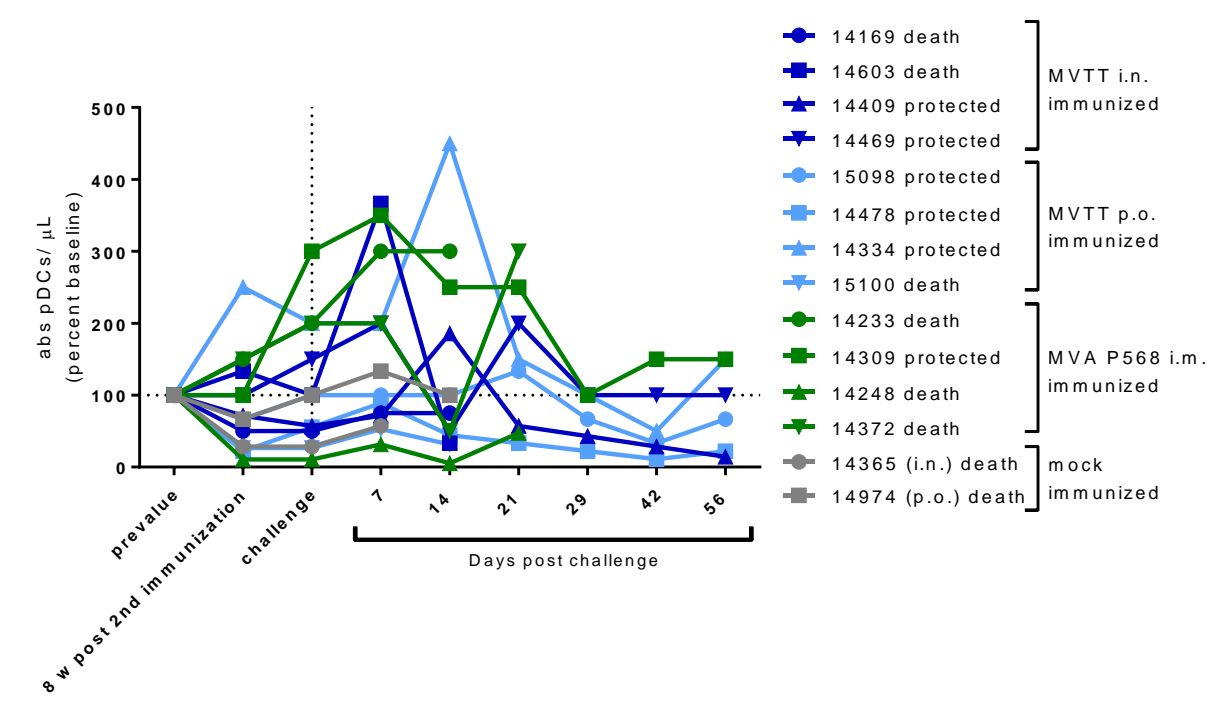

Figure 3-22. Absolute cell numbers of plasmacytoid dendritic cells (pDCs) in vaccination study II are shown over time. Absolute cell numbers are given in percent baseline. abs, absolute cell numbers; dpc, days post challenge; i.n., intranasal; i.m., intramuscular; MVA, modified vaccinia virus Ankara; MVTT, modified vaccinia virus Tiantan; p.o., per oral; P, passage, w, weeks.

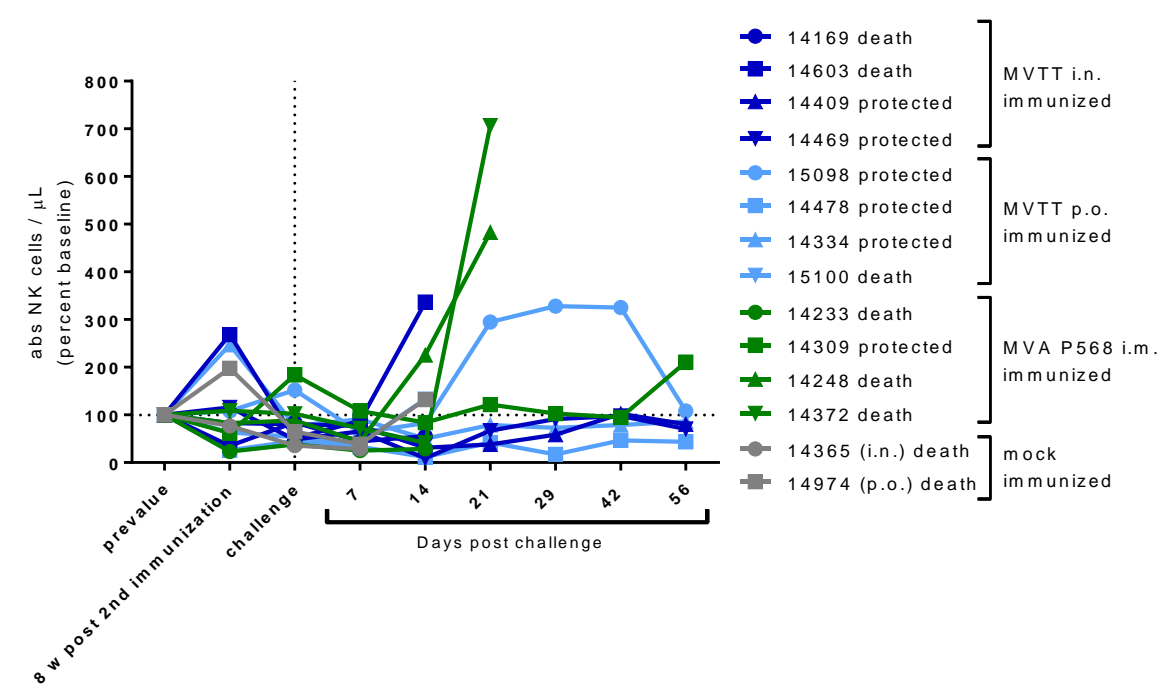

Figure 3-23. Absolute cell numbers of NK cells in vaccination study II are shown over time. Absolute cell numbers are given in percent baseline. abs, absolute cell numbers; dpc, days post challenge; i.n., intranasal; i.m., intramuscular; MVA, modified vaccinia virus Ankara; MVTT, modified vaccinia virus Tiantan; p.o., per oral; P, passage, w, weeks. 


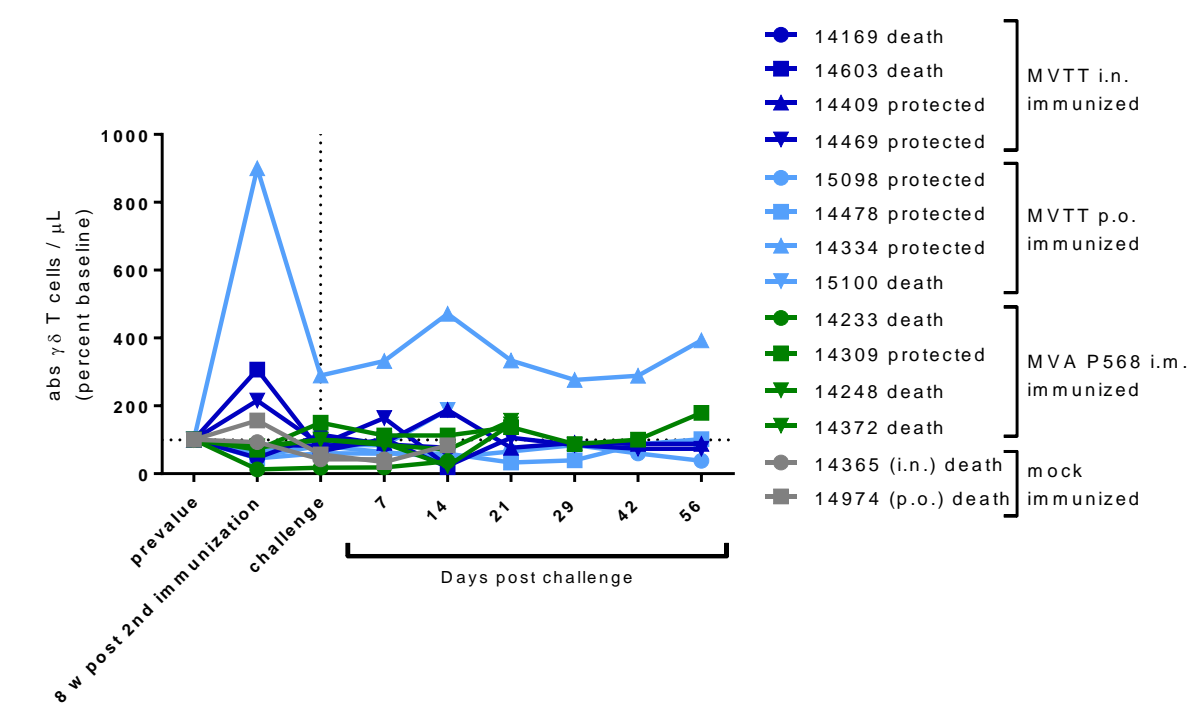

Figure 3-24. Absolute $\gamma \delta \boldsymbol{T}$ cells in vaccination study II are shown over time. Absolute cell numbers are given in percent baseline. abs, absolute cell numbers; dpc, days post challenge; i.n., intranasal; i.m., intramuscular; MVA, modified vaccinia virus Ankara; MVTT, modified vaccinia virus Tiantan; p.o., per oral; P, passage, w, weeks.

\section{Longitudinal phenotyping of adaptive immune cells}

\section{Longitudinal analysis of $\mathrm{CD3}^{+}, \mathrm{CD4}^{+}$and $\mathrm{CD8}^{+} \mathrm{T}$ cell numbers}

In absolute $\mathrm{CD}^{+}$, (Figure 3-25), $\mathrm{CD}^{+}$(Figure 3-26), $\mathrm{CD}^{+}$(Figure 3-27) and $\mathrm{CD} 4^{+} \mathrm{CD} 8^{+}$ (Figure 3-28) T cell numbers were no differences observed during immunization. The poxvirus infection did also not have a strong effect cell numbers or frequencies (data not shown) of $\mathrm{CD}^{+}, \mathrm{CD}^{+}, \mathrm{CD}^{+}$and $\mathrm{CD} 4^{+} \mathrm{CD} 8^{+} \mathrm{T}$ cells. 

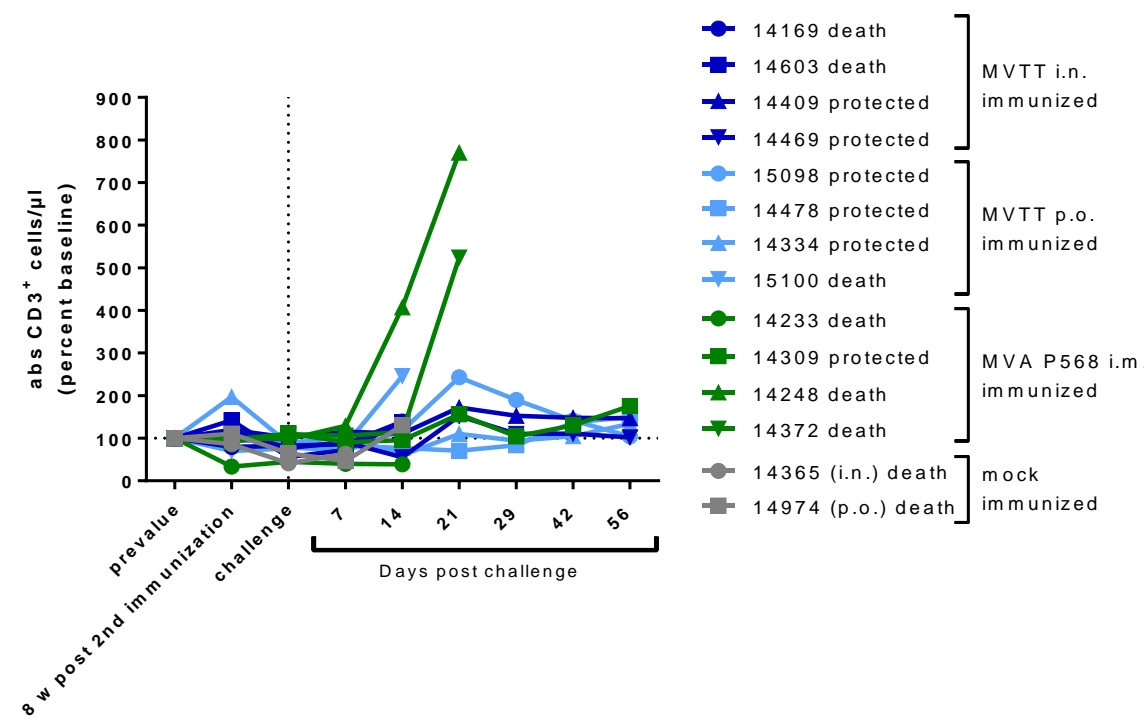

Figure 3-25. Absolute cell numbers of $\mathrm{CD3}^{+} \mathbf{T}$ cells in vaccination study II are shown over time. Absolute cell numbers are given in percent baseline. abs, absolute cell numbers; dpc, days post challenge; i.n., intranasal; i.m., intramuscular; MVA, modified vaccinia virus Ankara; MVTT, modified vaccinia virus Tiantan; p.o., per oral; P, passage, w, weeks.

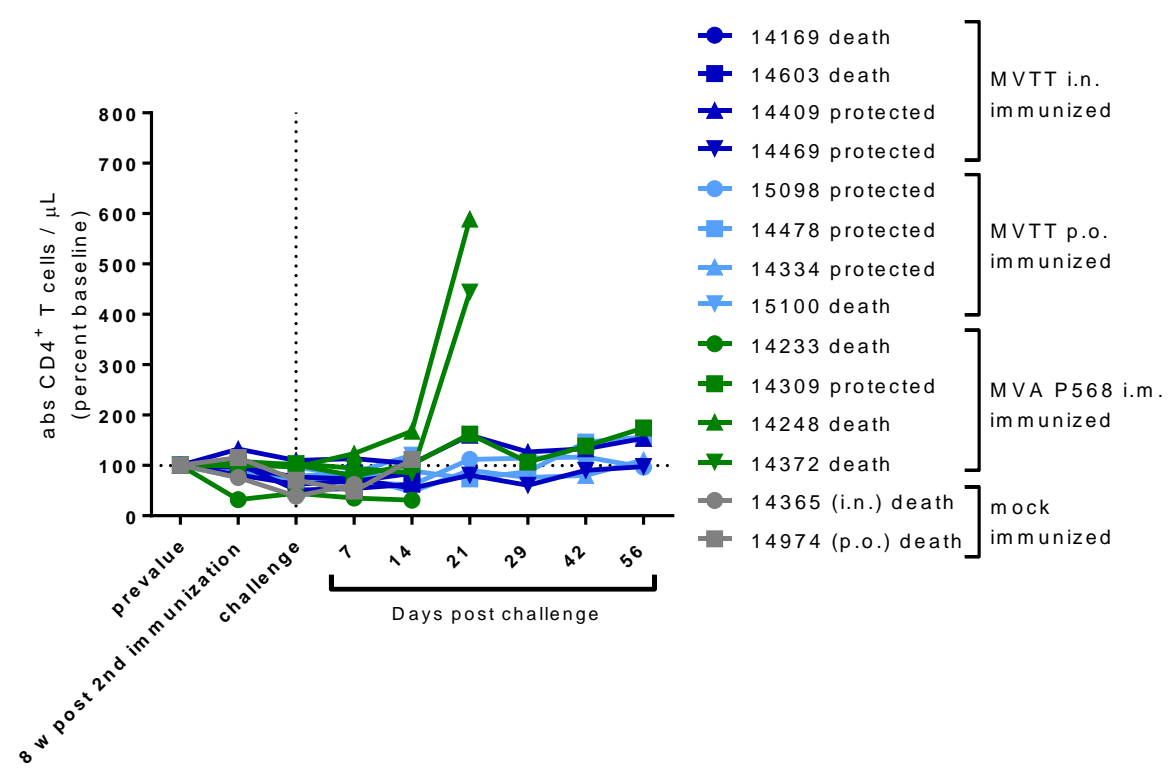

Figure 3-26. Absolute cell numbers of $\mathrm{CD4}^{+} \mathbf{T}$ cells in vaccination study II are shown over time. Absolute cell numbers are given in percent baseline. abs, absolute cell numbers; dpc, days post challenge; i.n., intranasal; i.m., intramuscular; MVA, modified vaccinia virus Ankara; MVTT, modified vaccinia virus Tiantan; p.o., per oral; P, passage, w, weeks. 


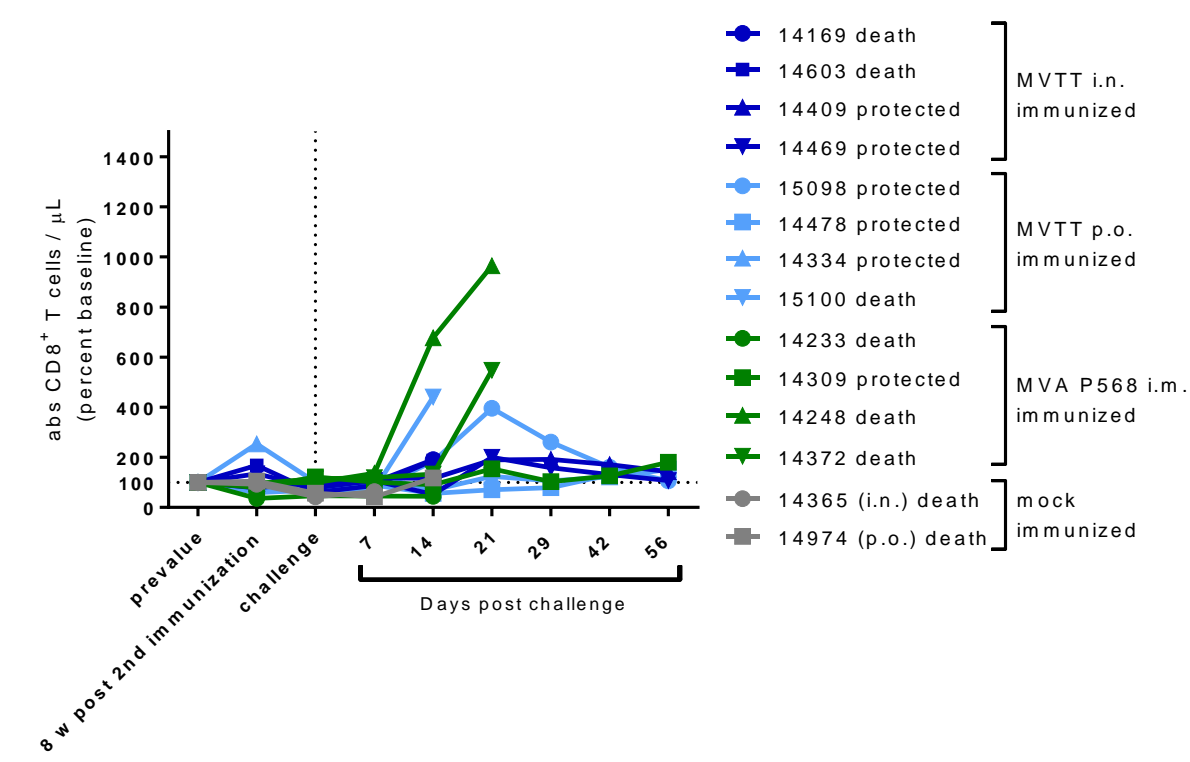

Figure 3-27. Absolute cell numbers of $\mathbf{C D 8}^{+} \mathbf{T}$ cells in vaccination study II are shown over time. Absolute cell numbers are given in percent baseline. abs, absolute cell numbers; dpc, days post challenge; i.n., intranasal; i.m., intramuscular; MVA, modified vaccinia virus Ankara; MVTT, modified vaccinia virus Tiantan; p.o., per oral; P, passage, w, weeks.
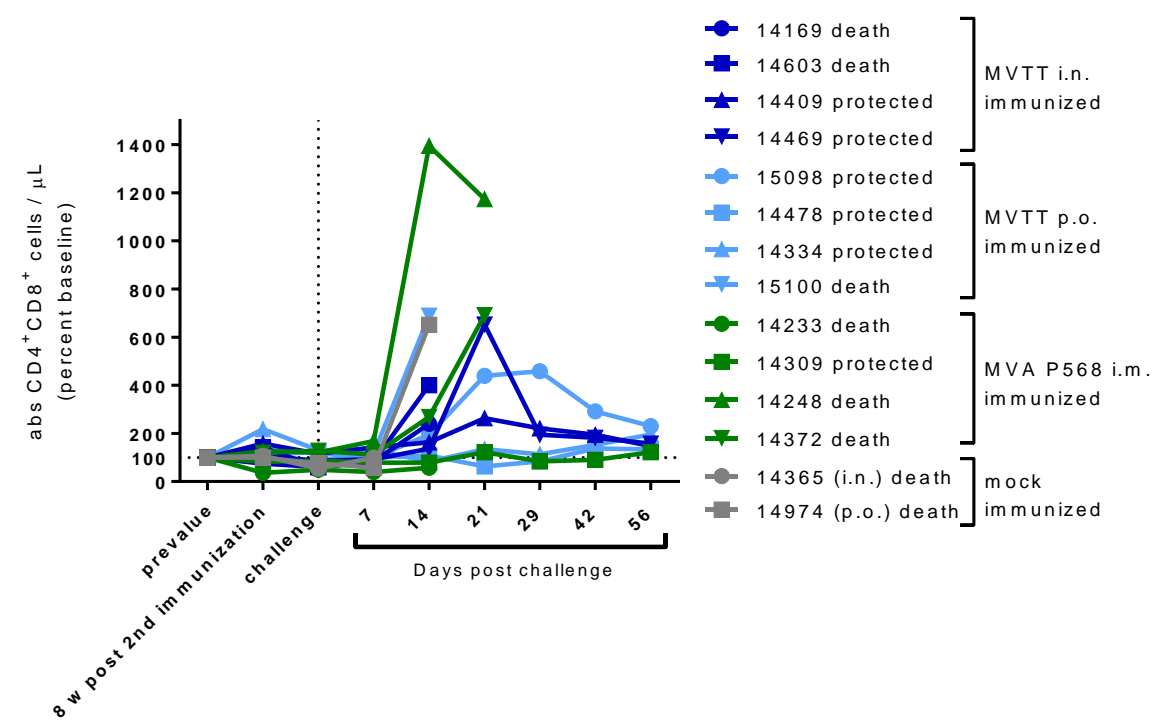

Figure 3-28. Absolute cell numbers of $\mathrm{CD4}^{+} \mathrm{CD8}^{+}$cells in vaccination study II are shown over time. Absolute cell numbers are given in percent baseline. abs, absolute cell numbers; dpc, days post challenge; i.n., intranasal; i.m., intramuscular; MVA, modified vaccinia virus Ankara; MVTT, modified vaccinia virus Tiantan; p.o., per oral; $\mathbf{P}$, passage, w, weeks.

\section{Longitudinal analysis of $\mathrm{CD}^{2} \mathrm{O}^{+} \mathrm{B}$ cells cell numbers}

Absolute $\mathrm{CD} 20^{+} \mathrm{B}$ cell numbers remained below or at baseline during the observation period in the majority of the animals (Figure 3-29). Only, in two MVA i.m. immunized animals (\#14248 and \#14372) the cell numbers of CD20+ B cells increased shortly before animals be- 
came moribund. Between groups there were no significant differences detected at the day of challenge.

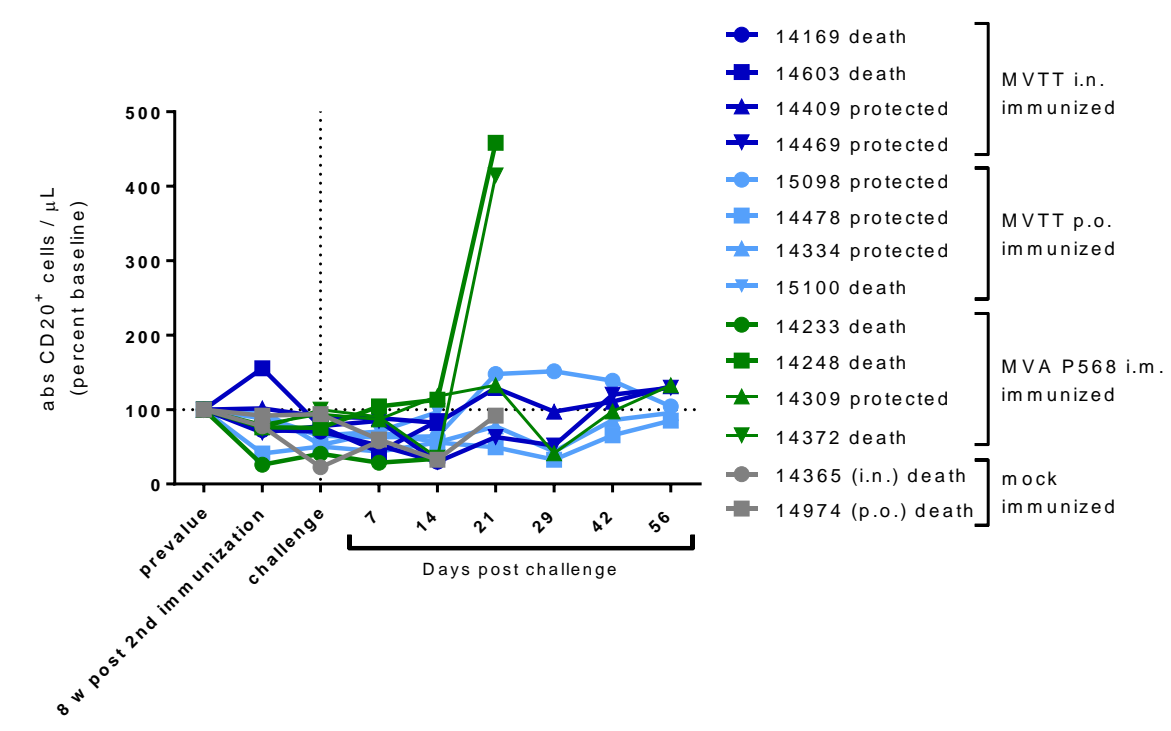

Figure 3-29. Absolute cell numbers of $\mathrm{CD20}^{+} \mathrm{B}$ cells in vaccination study II are shown over time. Absolute cell numbers are given in percent baseline. abs, absolute cell numbers; dpc, days post challenge; i.n., intranasal; i.m., intramuscular; MVA, modified vaccinia virus Ankara; MVTT, modified vaccinia virus Tiantan; p.o., per oral; P, passage, w, weeks

\section{Longitudinal analysis of the expression of activation marker $\mathrm{CD86}^{+}$on $\mathrm{CD}^{++}, \mathrm{CD14}^{+}$ $\mathrm{CD}^{+}{ }^{+}$and $\mathrm{CD}^{+}{ }^{+}$cells}

Expression of $\mathrm{CD}^{+} 6^{+}$was assessed on $\mathrm{CD} 14^{++}$(Figure 3-30), $\mathrm{CD} 14^{+} \mathrm{CD} 16^{+}$(Figure 3-31) and $\mathrm{CD}_{16}{ }^{+}$cells (Figure 3-32). The frequencies of the activated cells dropped after immunization but increased strikingly after challenge at day 21 . Thereafter the percentages decreased again and stayed below the baseline. Between groups there were no significant differences detected at the day of challenge. 


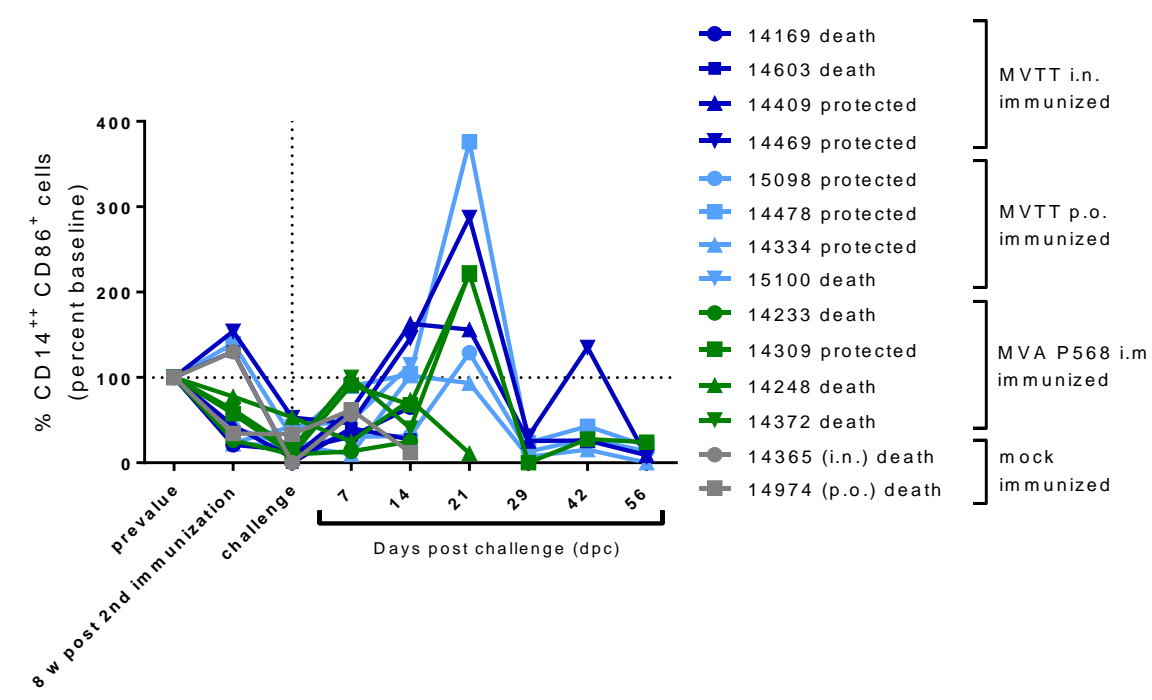

Figure 3-30. Percentages of $\mathrm{CD86}^{+}$cells of $\mathrm{CD}^{++}$cells in vaccination study II are shown over time. Percentages of cell numbers are given in percent baseline. dpc, days post challenge; i.n., intranasal; i.m., intramuscular; MVA, modified vaccinia virus Ankara; MVTT, modified vaccinia virus Tiantan; p.o., per oral; P, passage, w, weeks.

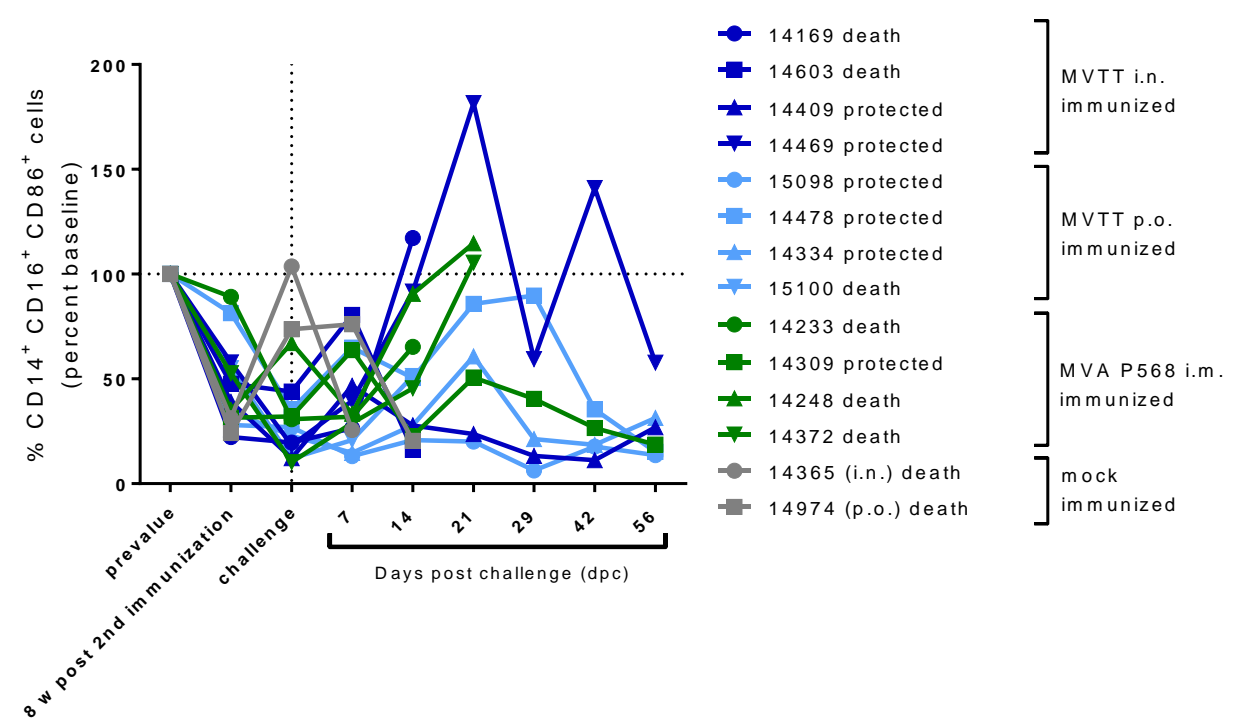

Figure 3-31. Percentages of $\mathrm{CD86}^{+}$cells of $\mathrm{CD14}^{+} \mathrm{CD16}^{+}$cells in vaccination study II are shown over time. Percentages of cell numbers are given in percent baseline. dpc, days post challenge; i.n., intranasal; i.m., intramuscular; MVA, modified vaccinia virus Ankara; MVTT, modified vaccinia virus Tiantan; p.o., per oral; P, passage, w, weeks. 


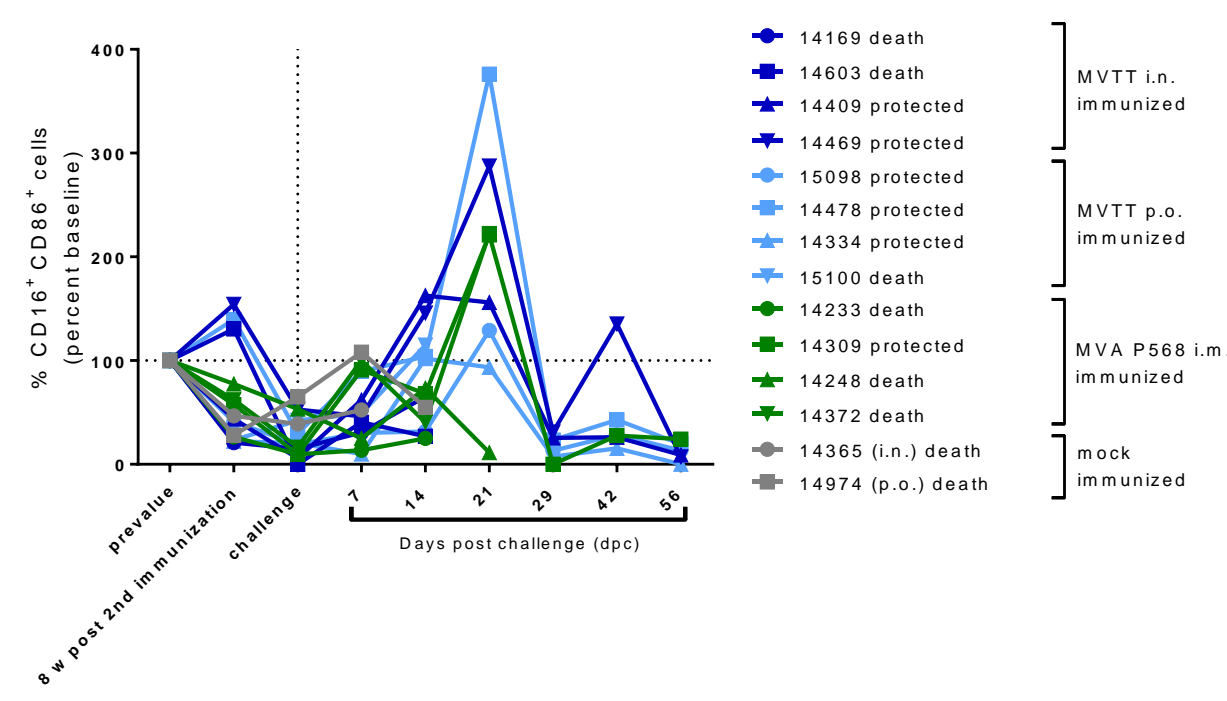

Figure 3-32. Percentages of $\mathrm{CD86}^{+}$cells of $\mathrm{CD16}^{+}$cells in vaccination study II are shown over time. Percentages of cell numbers are given in percent baseline. dpc, days post challenge; i.n., intranasal; i.m., intramuscular; MVA, modified vaccinia virus Ankara; MVTT, modified vaccinia virus Tiantan; p.o., per oral; $\mathbf{P}$, passage, w, weeks.

\section{No significant differences in $T$ and $B$ cell numbers and their expression of certain activa- tion markers}

No significant difference between vaccination groups in activation markers (e.g. CD69, HLADR and CD80) expressed on T and B cells were detected at the day of challenge. The course of the cell frequencies was thus similar to the parental $\mathrm{T}$ and $\mathrm{B}$ cell numbers (data not shown).

\subsubsection{T cell proliferation assay}

\section{Proliferating $\mathbf{T}$ cells over time}

$\mathrm{CD}^{+} \mathrm{T}$ cells as well as their subpopulations $\mathrm{CD} 4^{+}, \mathrm{CD} 4^{+} \mathrm{CD} 8^{+}$and $\mathrm{CD} 8^{+} \mathrm{T}$ cells were analyzed for their proliferating capacity after stimulation with orthopoxvirus antigen by evaluation of percentages of $\mathrm{CFSE}^{-}$cells. Before immunization hardly any proliferating cells were detected. No significant differences between the vaccination groups were observed during the observation period (no data shown).

Frequencies of $\mathrm{CD}^{+} \mathrm{CFSE}^{-}, \mathrm{CD}^{+} \mathrm{CD}^{+}, \mathrm{CD}^{+} \mathrm{CD}^{+}$, and $\mathrm{CD} 3{ }^{+} \mathrm{CD} 4{ }^{+} \mathrm{CD} 8^{+}$cells (Figure 3$33 \mathrm{~A}, 33 \mathrm{~B}, 34 \mathrm{~A}$ and $34 \mathrm{~B}$ ) were increased at 4-weeks post first immunization in protected and unprotected animals, whereas the frequencies of $\mathrm{CD}^{+} \mathrm{CFSE}^{-}$cells in mock immunized animals were also increased at that time point (Figure $3-33 \mathrm{~A}$ ). $\mathrm{CD}^{+} \mathrm{CD}^{+} \mathrm{CFSE}^{-}$cell frequencies (Figure 3-33B) increased at 8-weeks post second immunization. In the two protected animals \#14478 and \#14334 of the MVTT p.o. immunized group those frequencies slightly increased longitudinally even after challenge. 


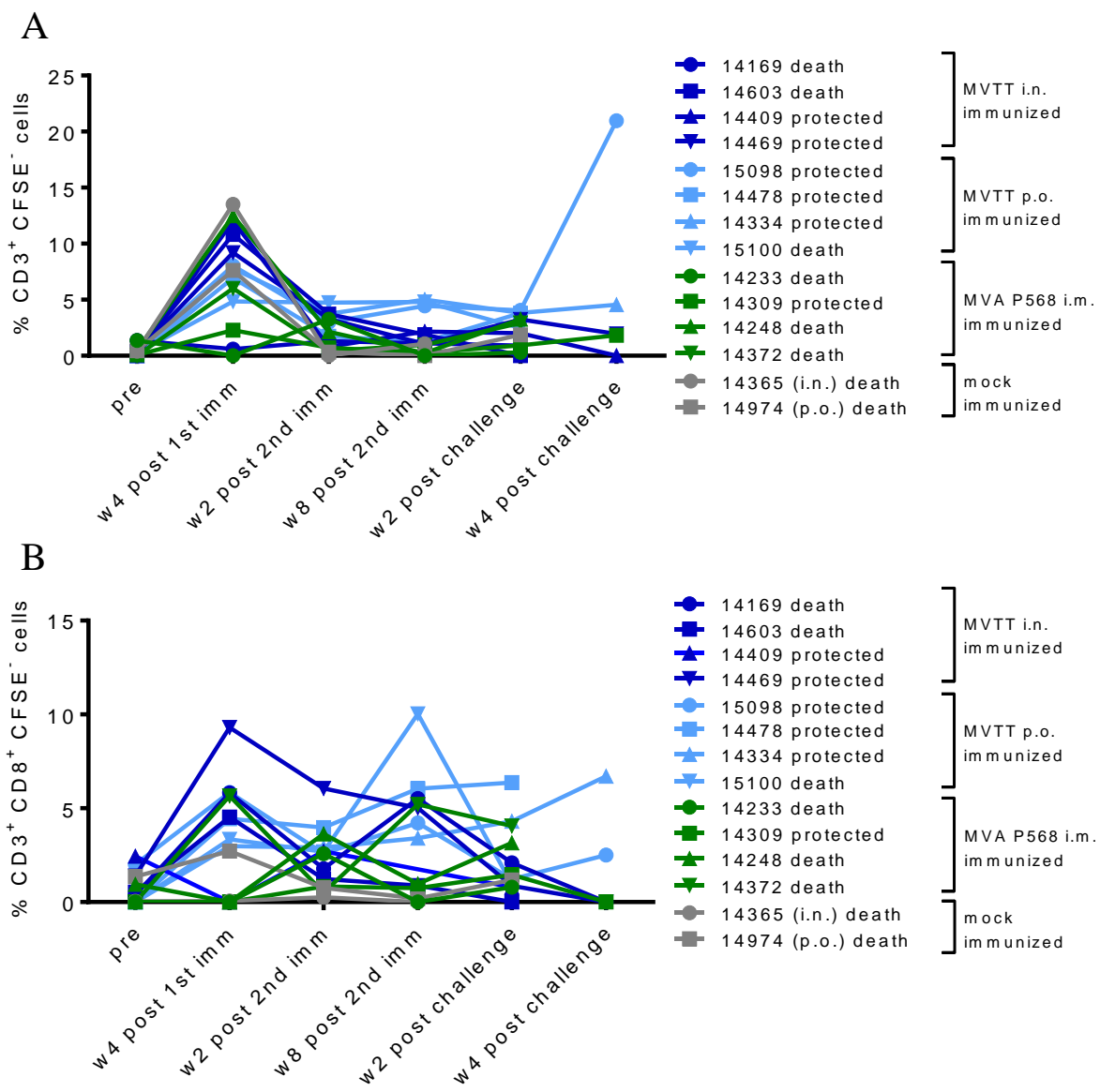

Figure 3-33. Proliferating $\mathrm{CD3}^{+}$and $\mathrm{CD3}^{+} \mathrm{CD8}^{+}$cells (CFSE-) frequencies were measured and increased at one time point (4-weeks post first immunization). PBMCs were labeled with the fluorescent dye CFSE to analyze T cell proliferation. PBMCs were stimulated with MVA for 6 days. Subsequently PBMCs were stained for flow cytometric analyses. Results of the MVA-stimulated samples are given as \%CFSE- cells of A) $\mathrm{CD}^{+}$or B) $\mathrm{CD}^{+} \mathrm{CD}^{+}$cells. death, animals were not protected and died after calpox virus challenge; imm, immunization; i.m., intramuscular; i.n., intranasal; MVA, modified vaccinia virus Ankara; MVTT, modified vaccinia virus Tiantan; P, passage; p.o., per oral; protected, animals were protected, w, weeks.

$\mathrm{CD}^{+} \mathrm{CD}^{+} \mathrm{CD}^{+} \mathrm{CFSE}^{-}$cells slightly increased longitudinally in MVTT vaccination groups in protected as well as cells of the unprotected animals (Figure 3-34B), while these cells in the mock immunized animals did not proliferate. Eight weeks post second immunization ( 2 week before challenge) the MVTT vaccinees had higher $\mathrm{CD} 3{ }^{+} \mathrm{CD} 4^{+} \mathrm{CD} 8^{+} \mathrm{CFSE}-$ cell frequencies than MVA vaccinees and mock immunized animals. Highest cell frequencies were detected in one protected MVTT vaccinee (\#15098) 4-weeks post challenge. The proliferative activity was not associated with the protection of the animals from the calpox virus challenge. After challenge, $\mathrm{CD}^{+} \mathrm{CD}^{+} \mathrm{CD}^{+} \mathrm{CFSE}^{-}$cells did not proliferate in one protected animal (\#14309) and in some unprotected animals an increase of cell frequencies was measured (\#14248, \#14603 and \#15100). 


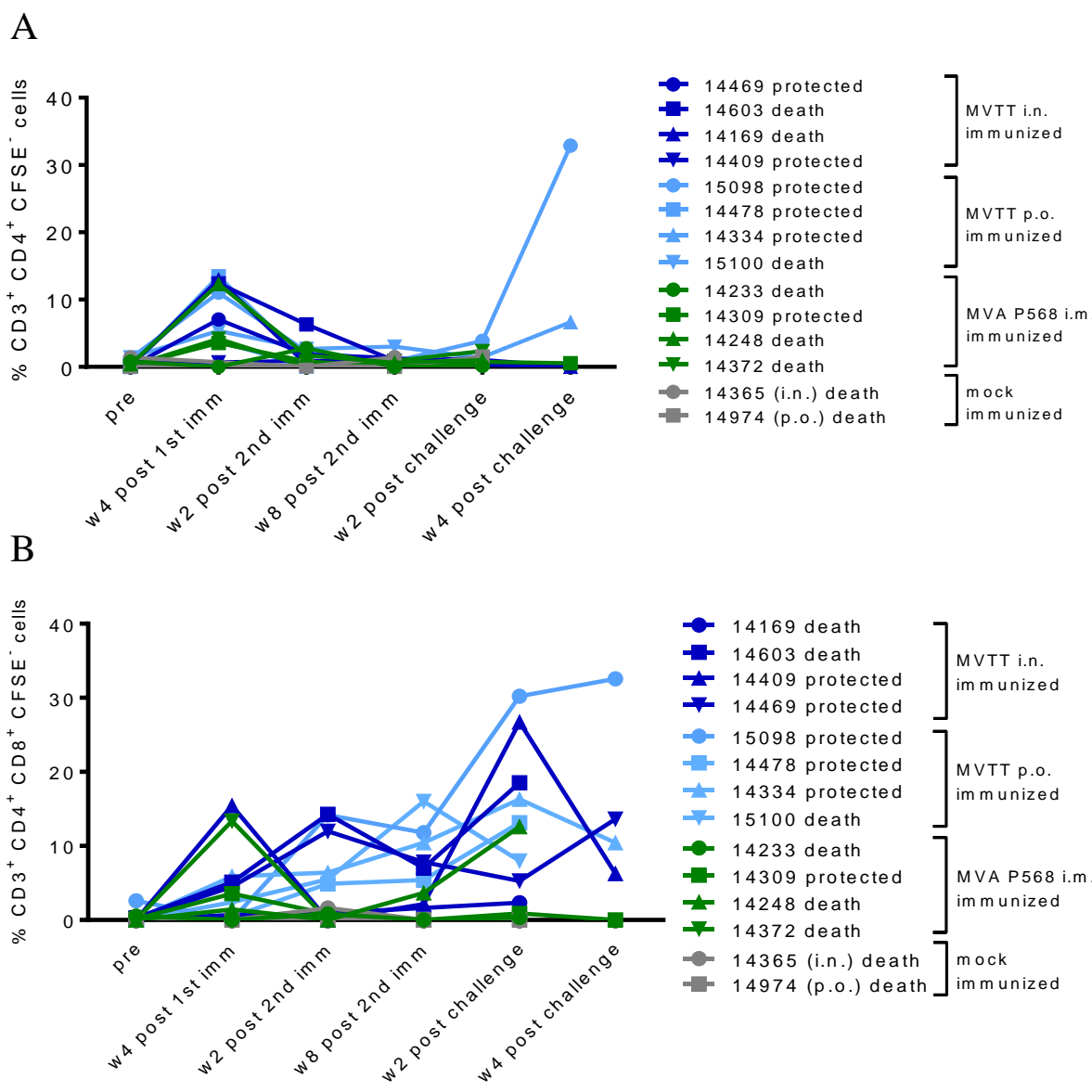

Figure 3-34. Frequencies of $\mathrm{CD3}^{+} \mathrm{CD}^{+} \mathrm{CFSE}^{-}$and $\mathrm{CD3}^{+} \mathrm{CD4}^{+} \mathrm{CD8}^{+} \mathrm{CFSE}^{-}$cells over time. $\mathrm{T}$ cells of the control group (mock immunized animals) did not proliferate. A) $\mathrm{CD}^{+} \mathrm{CD}^{+} \mathrm{CFSE}^{-}$cells. B) $\mathrm{CD} 3{ }^{+} \mathrm{CD} 4{ }^{+} \mathrm{CD}^{+} \mathrm{CFSE}^{-}$cell frequencies. $\mathrm{T}$ cell proliferation in blood was analyzed at different time points by labeling PBMCs with CFSE. PBMCs were labeled with the fluorescent dye CFSE to analyze T cell proliferation. PBMCs were stimulated with MVA. Subsequently PBMCs were stained for flow cytometric analyses. Results of the MVA-stimulated samples are given as \% of CFSE- of CD3 $3^{+} \mathrm{CD} 4^{+}$or $\mathrm{CD}^{+} \mathrm{CD} 8^{+}$ cells. death, animals were not protected and died after calpox virus challenge; imm, immunization; i.m., intramuscular; i.n., intranasal; MVA, modified vaccinia virus Ankara; MVTT, modified vaccinia virus Tiantan; P, passage; p.o., per oral; protected, animals were protected, w, weeks. 


\section{DISCUSSION}

\subsection{Pathogenesis study}

\subsubsection{Following intranasal application calpox virus enters through the nasal epithe- lium}

In 2010 the calpox virus marmoset model was first described offering a new non-human primate model to study OPXV pathogenesis, therapy and prophylaxis (Kramski et al., 2010). However, there have only been reports about the severe pathological alterations in the late stage of the disease caused by that orthopoxvirus (Kramski et al., 2010; Mätz-Rensing et al., 2012). Therefore, a study was designed in which serial necropsies were carried out during the asymptomatic phase of calpox virus infection in CMs. At each defined time point, a broad spectrum of organs was collected. In parallel to the virological analyses, organs were analyzed histologically for the occurrence of alterations by the Pathology Unit of the DPZ. One aim (I) was to determine primary target cells and organs of infection as well as to determine the portal of viral entry. (II) Another aim was to shed light on the early viral dissemination and the pathological sequelae of infection by detection of replicating calpox virus and calpox viral DNA in different organs and blood. In addition, cells that replicate the virus should be identified by flow cytometry.

Previous studies have shown that very low doses of calpox virus applied intranasally were sufficient for the infection of CM when compared to doses used in common OPXV models (Kramski et al., 2010). Since infection of the upper respiratory tract closely resembles the natural infection route of smallpox via aerosols, all animals were inoculated intranasally. Virus containg aerosols are inhaled, thereby passing the nasal, oral, and, pharyngeal mucosae or, if inhaled deeper reaching the lung and causing disease at this location (Barnewall et al., 2012; Fenner et al., 1988b). Therefore, these tissues could be targets of initial replication.

It is known that the initial site of the OPXV infection is the skin, a mucosal surface or the respiratory tract (Munoz, 2014a). For the calpox/marmoset model it was assumed that the nasal epithelium and the nasal-associated lymphoid tissue (NALT) represent the principal target for primary infection (Mätz-Rensing et al., 2012).

To increase the likelihood for the detection of even minute quantities of virus at the earliest time point of euthanasia, i.e. day 3 , approximately $3.5 \times 10^{5}$ PFU were administered. By qRTPCR and endpoint dilution assay, virus was detected in nasal mucosa and tongue at this early time point. Highest viral loads were found in nasal mucosa (epithelial layer of the mucous membranes) in the nose by both assays. Animals sacrificed at later time points after viral in- 
oculation received a 42-fold lower dose which had reliably infected 16 animals from unrelated studies (Kramski et al., 2010). Under these conditions in animals sacrificed at 5 dpi, only lowlevel calpox viral DNA was detectable in the nasal mucosa (1/3 CM) and two adjacent organs, but no infectious virus was recovered. This also indicated viral replication at the site of inoculation and was corroborated by high viral levels in the nasal mucosa of one out of three animals euthanized at $7 \mathrm{dpi}$. All these findings underline that nasal epithelial cells are the principle target cells for calpox virus. The results of the immunohistochemical analyses conducted by scientists of the DPZ Pathology unit demonstrated that the nasal epithelial cells serve as the portal of viral entry. Viral antigen was detected within inclusion bodies in this location at 3 dpi (Schmitt et al., 2017). Additionally, first alterations in the nasal ciliated epithelium were visible as well as immunohistochemical reactivity in respiratory epithelium at this early time point (Schmitt et al., 2017).

The nasal-associated lymphoid tissue (NALT) as well as the nasal epithelium have been assumed to serve as the principal target for primary infection in this model (Mätz-Rensing et al., 2012). The NALT is functionally active against infectious agents such as airborne transmitted viruses (e.g. VARV) or other pathogens and contains many immunocompetent cells like B cells, dendritic cells, macrophages and T cells (Bienenstock and McDermott, 2005). Furthermore, the NALT is rich in microfold cells. M cells take up viral antigen and transport them to the immune cells. Dendritic cells with captured antigen will then migrate to the draining lymph node and present the antigen to T cells (Xu et al., 2014).

\subsubsection{Identification of calpox virus replicating immune cells}

To identify cell subsets in blood harbouring calpox virus a flowcytometric assay was adapted for the analysis of purified PBMCs and buffy coat. An anti-VACV hyperimmune serum cross-reacting with calpox was used for viral detection. As no autologous preinfection values were available positivity was assessed by simultaneously analyzing blood from uninfected CM. Calpox virus antigen was observed in $\mathrm{CD}^{+} \mathrm{CD} 20^{+}$cells in two of three animals and in intermediate $\mathrm{CD} 14^{+} \mathrm{CD} 16^{+}$monocytes in one of three animals by day 3 post infection (high dose-infected animals). For cynomolgus macaques infected intravenously with the cowpox virus, it is known that especially monocytes and granulocytes became infected (Song et al., 2013b), and to a lesser extent, B cells, NK cells and T cells. In this study, calpox virus antigen was mainly present in $\mathrm{CD} 20^{+} \mathrm{B}$ cells and $\mathrm{CD}^{+} \mathrm{CD} 20^{+}$cells. Only a small proportion of neutrophils stained positive for calpox antigen in one animal of the day-12 group (Figure 3-5) while this subset was negative in all the other animals tested. By contrast, in monocytes 
$\left(\mathrm{CD} 14^{+} \mathrm{CD} 16^{-}, \mathrm{CD} 14^{+} \mathrm{CD} 16^{+}\right.$and $\left.\mathrm{CD} 14^{+} \mathrm{CD} 16^{-}\right)$calpox virus antigen was readily detected at 12 dpi. Levels of infected $\mathrm{CD}^{+} \mathrm{T}$ cells and NK cells were lower compared to those of monocytes and $\mathrm{CD} 20^{+} \mathrm{B}$ cells. With respect to infected immune cells in blood, $\mathrm{CD} 3{ }^{+} \mathrm{CD} 20^{+}$cells and monocytes appeared to be the preferred subset of calpox virus replication early in infection whereas the spectrum of infected cell types broadened in the late preclinical stage.

These findings are in agreement with a study of VACV WR infection of human PBMCs (Sanchez-Puig et al., 2004). When macrophages become infected, their ability to act as accessory cells to activate T-cells or natural killer (NK) cells and secrete cytokines is blocked which is of advantage for the virus (Smith and Kotwal, 2002). Perhaps the virus infects the immune cells that play an important role in host defense like NK cells in early (first three days) host immune response after poxvirus infection. The latter implies that a $\mathrm{T}$ cell response seems to play a more important role in early rather than the late phase of primary, acute infections (Gordon et al., 2011; Xu et al., 2004a).

A novel finding was the detection of calpox virus antigen in $\mathrm{CD}^{+} \mathrm{CD} 20^{+}$cells. To my knowledge, nothing is known about $\mathrm{CD}^{+} \mathrm{CD} 20^{+}$cells in $\mathrm{CM}$ so far and further research is needed to clarify their function. The existence of $\mathrm{CD} 3{ }^{+} \mathrm{CD} 20^{+}$cells have been controversely discussed and were thought to be an artifact of flow cytometry (Henry et al., 2010). They were also described as a storage-dependent phenomenon (Nagel et al., 2014). By contrast, according to more recent studies (Neumann et al., 2015; Schuh et al., 2016) $\mathrm{CD}^{+} \mathrm{CD} 20^{+} \mathrm{T}$ cells seem to represent a true population, pervading lymphatic organs and the cerebrospinal fluid. Functional properties of human $\mathrm{CD}^{+} \mathrm{CD} 20^{+} \mathrm{T}$ cells comprise the production of different cytokines (IL-4, IL-17, IFN- $\gamma$, TFN- $\gamma$ ). They also respond to multiple sclerosis disease modifying drugs (Schuh et al., 2016).

\subsubsection{Comparison of methods for the detection of calpox virus in blood samples}

Calpox virus levels were determined in blood by different methods, i.e. detection of the calpox virus antigen by flow cytometry, by real-time PCR and by plaque assay. qRT-PCR and calpox virus antigen staining by flow cytometry were more sensitive than virus isolation. When samples from the day- 3 animals were analyzed by the same techniques in parallel, calpox virus DNA was detected in only one of three animals by real-time PCR, in two of three animals the fraction of $\mathrm{CD}^{+} \mathrm{CD} 20^{+}$cells was positive for calpox virus antigen by flow cytometry, but no virus could be isolated from buffy coat. In animals exhibiting highest percentages of calpox virus positive cells as determined by flow cytometry, also highest levels of calpox DNA copy numbers were observed. 
Real-time PCR and poxvirus antigen staining by flow cytometry were the most time-efficient methods. Moreover, staining for calpox virus antigen by flow cytometry has the advantage that antigen expression in different immune cells can be tested in one approach. Because of a lower background staining (baseline) in samples from the controls animals, the calpox virus antigen staining by flow cytometry with cells from buffy coat is preferred to PBMCs.

\subsection{4. (II) The early dissemination and pathological sequelae of infection}

The pathogenesis study provides evidence that early dissemination starts in the nasal mucosa. Calpox viral DNA and replicating calpox virus were already detectable in the nasal mucosa of two animals of the high-dose group sacrificed at 3 dpi. Also, few organs adjacent to the inoculation site were either positive for viral DNA or infectious virus. Presence of virus in tonsil, tongue, esophagus and lung most likely resulted from passing or swallowing of viruscontaining droplets and in the case of lung from deep inhalation. The presence of viral DNA in the eye was unexpected, but might be explained by smear infection. In the animals infected with the lower calpox virus dose and sacrificed at day 5, low levels of viral DNA were detected in the nasal mucosa, tonsil and submandibular lymph node. Parallel analyses of the organs by immunohistochemistry for the detection of calpox virus antigen (Schmitt et al., 2017) suggested that trafficking of infected macrophages presumably led to a secondary replication in the submandibular lymph nodes (Schmitt et al., 2017). Nasal mucosa was also positive for virus at day 7 in one animal (\#14483) which exhibited high viral loads in that organ. Those were a couple of magnitudes higher compared to viral loads in the other organs of this animal or the loads in the other two animals of that group being either positive for viral DNA and/or infectious virus. Overall, in the day-7 animals only mild histological lesions were observed (Schmitt et al., 2017).

In addition to the analyses of viral loads in a multitude of organs, also blood was investigated to define at which point in time viremia occurred. One animal of the day-3 high dose group presented with low level viremia based on viral DNA copy numbers (100 copies per $\mu 1)$. This animal was one of the two animals, which had comparable high levels of viral DNA and infectious virus in the nasal mucosa. Thus, considerable viral replication at the portal of viral entry did not automatically resulted in viremia. Rather, early systemic viral spread might have been contained in the organ-positive, but blood-negative animal by short-lived innate immune responses. In the animals infected with the low calpox virus dose, viremia was only observed in two of three animals sacrificed at day 7. It can therefore be assumed that the onset of viremia is dose dependent since it started later in low-dose infected animals. Most of the animals 
became viremic at 10 and $12 \mathrm{dpi}$. The results of the endpoint dilution assay were in line with the results of the real-time PCR.

Not surprisingly, the highest calpox DNA copy numbers in blood were detected in all animals euthanized at the latest predefined time point (12 dpi). The immune system probably collapses around that day followed by death within a few days. Discrepancies between pathological and molecular biological findings (e.g. trachea was negative for all 12 dpi animals in immunohistochemistry and no histological lesions were found (Schmitt et al., 2017), but had detectable calpox virus DNA and particles, (see Figure 3-1D)) can be explained by the different locations of primary material/tissue of an organ analyzed by the different techniques. This suggests that even in the late preclinical stage of infection viral replication is still focal within an organ.

The preferred organs of calpox replication before appearance of clinical signs appear to be nasal mucosa, bone marrow, liver and generally the lymphoid tissues, as indicated by high viral loads, i.e. $10^{9}-10^{17}$ calpox DNA copy number/g tissue and $10^{7}-10^{9} \mathrm{TCID}_{50} / \mathrm{g}$ tissue. Lower levels of calpox virus were observed in the central nervous system (CNS), parotid gland, small intestine and stomach. These findings are consistent with other studies (Tucker, 2011). Overall, calpox virus replicated in a broad panel of organs tested, albeit to a different extent.

My own data suggests that the calpox virus initially replicated in the upper respiratory tract, i.e. the nasal mucosa. From there the virus probably reaches via lymphatics the adjacent lymphatic tissue (submandibular LN and tonsil) in which secondary replication takes place. Next, the virus is transported through the blood stream all over the body (day 7). Lower viral loads (by real-time PCR and plaque assay) were found in the central nervous system (CNS), parotid gland, small intestine, stomach, esophagus, parotid gland and vagina/testicle indicating that the calpox virus replicated less inefficiently in these organs. These findings are consistent with other studies (Tucker, 2011).

The viral dissemination/pathogenesis in the calpox virus/marmoset model is comparable to that of human smallpox infection (Fenner et al., 1988b), because it also starts in the respiratory mucosa, spreads then to the draining lymph nodes and finally disseminates via blood to other organs. The same way of distribution was observed in a study in which cynomolgus macaques were infected with aerosolized MPXV by using a head-only exposure chamber with a collision nebulizer (Zaucha et al., 2001). As in my study, the virus was detected in various organs in lethal MPXV infection. Moreover, Kramski and colleagues described the involvement of organs of the digestive tract in the calpox virus/marmoset model as opposed to human 
VARV infections (Fenner et al., 1988b; Kramski et al., 2010), which was also confirmed by this study. Following high-dose viral exposure, a monocytic cell-associated viremia was observed in one animal at 3 dpi. By day 7, virus was detectable in a broad spectrum of organs. Mainly epithelial cells and macrophages, but also endothelial cells were infected (Schmitt $e t$ al., 2017).

Because the last time point for predefined euthanasia (day 12) was very close to the time span of infection-related death, viral loads were highest compared to the earlier time point of necropsy. On that day, multiple immune cell subsets were also positive for calpox virus antigen. The broad cell tropism of the calpox virus is demonstrated in a study by Kramski and colleagues (Kramski et al., 2010). It has been shown that independent of the infection route and inoculated viral dose, high copy numbers of genomic viral DNA and virus-specific mRNA indicating active replication were found in a broad spectrum of organs and tissues (Kramski et al., 2010). Since VACV WR and MVA as well as the calpox virus seem to have also preferences for certain cell populations (Sanchez-Puig et al., 2004), this may indicate that factors other than host range genes like the accessibility and the amount of receptors, ability to internalize the virus and the metabolic state of the cell might influence the infection rate of PBMCs and immune cells.

In lethal human monkeypox virus (MPXV) and variola virus (VARV) infection virus was also found in various organs (Fenner et al., 1988c). It has been shown for VARV infections in cynomolgus macaques that viral replication was probably associated with organ dysfunction and multisystem failure of all the organs (Jahrling et al., 2004), which assumably was also the case in this study. In the calpox virus/marmoset model, all animals that developed clinical signs died which is in contrast to natural human VARV and MPXV infections, where only part of the diseased patients became moribund. Seroconversion was not observed in animals that died within 12 days (Kramski et al., 2010) and was not analyzed in this pathogenesis study. Obviously, when the survival time is too short, virus-specific IgM antibodies are not mounted until the time point of death.

In the calpox virus/marmoset model a clearly shorter time was reported between the first appearance of clinical signs until death, i.e. one or two days (Kramski et al., 2010), compared to human smallpox or MPXV infection. There, a period of two to five days was reported during which fever, malaise, prostration, headache and backache occurred that was followed by the development of a maculopapular rash. Animals of this study that were euthanized or died unexpectedly between 12 or $16 \mathrm{dpi}$, showed varying clinical signs (Schmitt et al., 2017). Two animals from the group for which euthanasia was predetermined at 12 dpi had only very few 
typical pox lesions on the skin, at mucocutaneous junctions and mild nasal discharge. The animals with clinical signs showed fatigue, anorexia, moderate to severe seromucous nasal discharge, and multiple pox lesions on skin and mucocutaneous junctions one to two days prior to death. Also, MPXV and VARV patients develop a more or less confluent rash (Fenner et al., 1988a; Fenner et al., 1988b; Fenner et al., 1988c; Jezek et al., 1987; Parker et al., 2007) which is lacking in the calpox virus infected monkeys (Kramski 2010). Only small, however multiple skin lesions distributed on the face, abdomen and thighs after i.n. infection with calpox virus occurred in CM (Kramski et al., 2010). Studies have already shown that even a high calpox viral dose did not lead to confluent skin lesions, which are characteristic for and previously demonstrated in macaques infected with MPXV and VARV (Hooper et al., 2004; Jahrling et al., 2004; Paluku and Szczeniowski, 1988; Stittelaar et al., 2005).

The morphology of the skin lesions induced by calpox virus differs from VARV or MPXV lesions. While in calpox infection skin lesions were flat and small in size (2-5 $\mathrm{mm}$ in diameter), VARV- or MPXV-associated lesions reach a diameter of up to $10 \mathrm{~mm}$. However, the size of the animal may also be related to the size of the skin lesions. A severe lymphadenopathy was found in calpox virus infection and represent a classic alteration in many OPXV infections. The lymphadenopathy is comparable to the clinical picture of cowpox virus (CPXV) and MPXV infections in humans and also in NHP, but is not observed in human smallpox (Fenner et al., 1988a; Heraud et al., 2006; Hooper et al., 2004; Sale et al., 2006).

\subsubsection{Virus titration and viral DNA quantification of organ homogenates}

The infectious virus titre of a tissue is known to be lower than the titre of the viral load since real-time PCR not only measures the replicating virus (like $\mathrm{TCID}_{50} / \mathrm{g}$ tissue) but also other viral particles. Results of endpoint dilution assay and real-time PCR mostly matched with the pathological finding e.g. nasal mucosa was negative for all 7 dpi animals, except in one animal (\#14483) with the highest viral load and replicating viral titre at 7 dpi. In a parallel processed dissertation (Schmitt, 2015), mild histological lesions were detected in this animal. Discrepancies between pathological and molecularbiological findings (e.g. trachea was negative for all 12 dpi animals in immunohistochemistry and no histological lesions were found, but had detectable viral load and was also positive in endpoint dilution assay, see Figure 31D) can be caused by using different primary material/tissue of an organ.

Expectedly, the highest $\mathrm{TCID}_{50} / \mathrm{g}$ tissue and calpox DNA copy number/g tissue were measured in 12 dpi animals, because of the close time to the expected moribund endpoint at $12 \mathrm{dpi}$. 
Since the comparison of the two assays (real-time PCR and endpoint dilution assay) shows that the bone marrow, nasal mucosa and submand. LN were strongly positive organs in both tests, the calpox virus consequently replicated efficiently in these organs. Low viral levels in both assays were determined in the central nervous system (CNS), parotid gland, small intestine and stomach, which indicate that the calpox virus replicated less efficiently in these organs. These findings are consistent with other studies (Tucker, 2011).

Both assays were positive at $3 \mathrm{dpi}$ for nasal mucosa and tongue, the highest values however, were measured in nasal mucosa (epithelial layer of the mucous membranes in the nose) in both assays. The highest viral load in 5 dpi animals (with the low inoculation dose) was also detected in the nasal mucosa in one of three animals, which indicates earliest replication of the virus in the nose for both inoculation doses. There is no evidence for a viremia at $5 \mathrm{dpi}$, because no infectious virus was detected and only few tissues were positive in of two of three animals. Calpox DNA copy numbers and infectious virus titres were found in a broad spectrum of organs from $7 \mathrm{dpi}$ to $12 \mathrm{dpi}$. In conclusion the viremia then starts between 5 and $7 \mathrm{dpi}$ after a local virus replication at the viral application site.

To sum up the results of the endpoint dilution assay and real-time PCR, the data provide evidence that the calpox virus first replicates where the virus is applied (in the nose) and evidence for viremia starting between 5 and 7 dpi is shown.

\subsection{6 (I) Portal of viral entry}

One of the aims was to determine the portal of viral entry. Only very mild histological alterations were detected at day 5, 7 and 10 as described in a dissertation conducted in parallel (Schmitt, 2015). Also, in almost all day-5 animals, no calpox virus DNA and no infectious calpox virus was detected. Therefore, a second study was performed where 3 more animals were infected with an approximately 42 -fold higher dose in order to increase sensitivity of the analyses.

There have only been reports about the severe pathological alterations of the late stage of the disease caused by calpox virus. Therefore, a study where serial necropsies were carried out during the asymptomatic phase of calpox virus infection in common CM (CM) was designed. At each defined time point, a broad spectrum of organs was collected and analyzed for the occurrence of alterations (see dissertation by Schmitt, 2015). The aim was to determine primary target cells and organs of infection as well as to determine the portal of viral entry (I). Another aim was to investigate the early dissemination and the pathological sequelae of infection (II) by detection of replicating calpox virus and calpox viral DNA in different organs 
(present study). In addition, the detection of replicating calpox virus and calpox virus DNA in blood was in focus. The aims of the study were to detect the start of viremia and to analyze by flow cytometry which are the preferred immune cell subsets for calpox infection. Studies have shown that in the calpox model when employing intranasal virus inoculation very low infectious doses are sufficient to establish infection compared to common OPXV models (Kramski et al., 2010). Therefore, in this study, animals were inoculated via the intranasal (i.n.) route. Also, the infection of the upper respiratory tract closely resembles the natural infection route of smallpox, which occurs via aerosols. Aerosols with an infectious virus are inhaled, exposed to the oral, nasal, pharyngeal mucosae or if inhaled deeper to the lung, could lead to disease in the lower respiratory tract (Barnewall et al., 2012; Fenner et al., 1988b).

It is known that the initial site of the orthopoxvirus (OPXV) infection is the skin, a mucosal surface or the respiratory tract (Munoz, 2014b). For the calpox/marmoset model it was assumed that the nasal epithelium and the nasal-associated lymphoid tissue (NALT) represent the principal target for primary infection when the virus is administered intranasally (MätzRensing et al., 2012). To understand the pathogenesis of the calpox virus infection in CM the pathogenesis study was conducted to analyze the early replication site. The detection of replicating calpox virus and viral DNA in the nasal mucosa three days after inoculation leads to the conclusion that calpox virus first multiplies at the inoculation site, the nasal mucosa. The portal of viral entry and the location of primary replication is thus the nasal mucosa (nose) when the virus is inoculated intranasally. Different findings underline the observation that nasal epithel cells are the principle target cells. First, viral DNA was also almost exclusively detectable in the nasal mucosa at 5 dpi when the low infectious dose was given. In addition, the results of the immunohistochemical analyzes presented in a dissertation conducted in parallel, show that the nasal epithelial cells serve as the principal target cells for primary replication since viral antigen within inclusion bodies was detected in that study at $3 \mathrm{dpi}$ (Schmitt, 2015). Additionally, first alterations in the nasal ciliated epithelium were visible as well as immunohistochemical reactivity in respiratory epithelium at 3 dpi.

The nasal mucosa is the location of viral entry. The nasal mucosa's immune system is the nasal-associated lymphoid tissue (NALT) that has been assumed to be the principal target for primary infection in this model (Mätz-Rensing et al., 2012). The NALT can protect against infectious agents such as airborne transmitted viruses (e.g. VARV) or other pathogens and contains many immunocompetent cells (e.g. B cells, dendritic cells, macrophages, $\mathrm{T}$ cells) (Bienenstock and McDermott, 2005). Furthermore, NALT consists of follicle-associated epithelium, the overlying epithelium with microfold $M$ cells. $M$ cells take up viral antigen and 
transport them to the immune cells. Dendritic cells with antigen will then migrate to the draining lymph node and present the antigen to T cells (Xu et al., 2014).

Consistently, the first targets for naturally acquired smallpox infection are the cells of the mucous membranes of the mouth, the nasal cavity, the oropharynx and the alveoli of the lungs. The findings in this study seem reasonable, because in other CPXV and ECTV species the replication site is the infected epithelia (Patel et al., 1986).

\subsubsection{Calpox virus detected in blood}

\section{Infectious virus in buffy coat and viral DNA quantification from whole blood}

Earliest calpox viral DNA in blood was detected in two of three animals at 7 dpi (\#14966 and \#14483). Therefore, supposably viremia starts between 5 dpi (when no viral DNA was detected) and $7 \mathrm{dpi}$ following inoculation with the lower viral dose $\left(8.3 \times 10^{3} \mathrm{pfu}\right)$. It is assumed that the dose was too low to cause viremia at 5 dpi animals, since all three animals at 5 dpi were negative in blood for viral DNA. Most of the animals became viremic after calpox infection at the following time points.

To increase the probability to track down the virus, the day-3 animals received an approximately 42 -fold higher dose $\left(3.5 \times 10^{5} \mathrm{pfu}\right)$ and one of three animals at 3 dpi became viremic. Earliest viral DNA in blood was found in one animal at 3 dpi (\#14484) (3.2.1.2 Viral DNA quantification from whole blood), however, this did not coincide with detection of replicating virus in buffy coat. This can be explained by the fact that the infectious virus titre in buffy coat is known to be lower than the titre of viral load since real-time PCR method is more sensitive. The real-time PCR measures the replicating virus as well as non-replicating viral particles.

The results of the plaque assay using buffy coat for cocultivation with sensitive indicator cells appeared to be valid when compared to the results of the real-time PCR at 7 dpi. Day $-5,-7$ and -10 group animals were not tested for replicating calpox virus in blood because the method was not established at that time point. Instead, the day-12 group was tested at 7, 10 and 12 dpi. The first replicating virus was detected in one of three animals (\#15095) of the day-12 group (animals were infected with $8.3 \times 10^{3}$ pfu calpox virus) at $7 \mathrm{dpi}$, but analysis for viral DNA was not done. However, calpox viral DNA was detected in two of three animals (\#14966 and \#14483) from the day-7 group.

Most of the animals became viremic at 10 and 12 dpi and at 10 dpi the replicating virus and calpox viral DNA increased because the end of the incubation period is at approximately 11 
days post infection. As expected, the highest calpox DNA copy numbers in blood were detected in all animals euthanized at the latest time point (12 dpi).

To sum up the results of testing blood for infectious virus as analyzed by plaque assay with buffy coat and for viral DNA by real-time PCR with whole blood, it can be postulated that a start of viremia (DNA copies in blood) is dose dependent. In this model viremia starts at 3 dpi (with the high inoculation dose) or between 5 and 7 dpi (with the low inoculation dose).

\subsubsection{Detection of calpox virus infection with anti-vaccinia virus (VACV) serum} The viremia starts at day 3 with the high dose $\left(3.5 \times 10^{5}\right.$ pfu calpox virus $)$

Calpox virus antigen positive $\left(\right.$ calpox $\left.^{+}\right)$cells were detected in cells from buffy coat and PBMCs. The detection of calpox ${ }^{+}$cells of $\mathrm{CD}^{+} \mathrm{CD} 20^{+}$cells in two of three animals and the detection of calpox ${ }^{+}$cells of intermediate $\mathrm{CD} 14^{+} \mathrm{CD} 16^{+}$monocytes in one of three animals of the 3-day group indicates the start of viremia at day 3. These results are in line with other findings. Therein, the calpox virus was detected in the blood of one of three animals at that time point by real-time PCR (4.1.7 Calpox virus detected in blood).

Early reports have indicated that VACV causes a cytopathic effect in human leukocytes and an infection of leukocytes has also been reported in monkeys. For cynomolgus macaques infected intravenously with cowpox virus, it is known that especially monocytes and granulocytes were positive for the virus (Song et al., 2013b). Monocytes and granulocytes/neutrophils were also the major populations that were stained positive for pox antigen, whilst frequencies of B cells, NK cells and T cells were low in cynomolgus macaques. However, in the pathogenesis study presented herein neutrophils were tested negative in day- 3 and only a very small proportion of neutrophils appeared to be positive in one day-12 animal. Monocytes $\left(\mathrm{CD} 14^{+} \mathrm{CD} 16^{-}, \mathrm{CD} 14^{+} \mathrm{CD} 16^{+}\right.$and $\left.\mathrm{CD} 14^{+} \mathrm{CD} 16^{-}\right)$were tested calpoxvirus antigen positive at 12 dpi. But their proportion was not as high as that of $\mathrm{CD} 20^{+} \mathrm{B}$ cells and of $\mathrm{CD}^{+} \mathrm{CD} 20^{+}$cells. To my knowledge, nothing is known about $\mathrm{CD}^{+} \mathrm{CD} 20^{+}$cells in $\mathrm{CM}$ so far and further research is needed to clarify their function in common CM (CM). Double positive $\mathrm{CD}^{+} \mathrm{CD} 20^{+}$cells are usually only detected in e.g. lymph nodes of rhesus macaques (Macaca mulatta), but not in blood. Schuh and colleagues reported that 3-5\% of the T cells in human blood express $\mathrm{CD} 20$ and that the $\mathrm{CD} 3{ }^{+} \mathrm{CD} 20^{+} \mathrm{T}$ cells produce different cytokines, pervade lymphatic organs and the cerebrospinal fluid. (Schuh et al., 2016). They also respond to MS disease modifying drugs. The existence of $\mathrm{CD}^{+} \mathrm{CD} 20^{+}$cells have been controverely discussed and were thought to be an artifact of flow cytometry in the past (Henry et al., 2010). 
They also have been described as a storage-dependent phenomenon (Nagel et al., 2014). Following recent studies (Neumann et al., 2015; Schuh et al., 2016) $\mathrm{CD}^{+} \mathrm{CD} 20^{+} \mathrm{T}$ cells do exist. The function of human $\mathrm{CD}^{+} \mathrm{CD} 20^{+} \mathrm{T}$ cells is the production of different cytokines (IL-4, IL17, IFN- $\gamma$ and TFN- $\gamma$ ), pervading lymphatic organs and the cerebrospinal fluid. They also respond to MS disease modifying drugs.

This study clearly demonstrated the presence of the calpox virus antigen in different immune cells (neutrophils, monocytes, T and B cells and NK cells) by flow cytometry. In marmostes infected for 12 days, multiple immune cell subsets were positive for calpox virus antigen. Furthermore, the results of this study point to a preference of calpox virus infecting certain cell types. Infection was detected in all tested immune cells (monocytes, T and B cells and NK cells) except for the neutrophils. Followed by $\mathrm{CD} 20^{+} \mathrm{B}$ cells and $\mathrm{NK}$ cells $\mathrm{CD} 3^{+} \mathrm{CD} 20^{+}$cells, the monocytes were most susceptible. $\mathrm{CD}^{+} \mathrm{T}$ cells were infected at very low frequencies. These findings are conform with the results of VACV WR infection of human PBMCs by Sanchez-Puig and colleagues (Sanchez-Puig et al., 2004). When macrophages get infected, their normal ability to act as accessory cells to activate T-cells or natural killer (NK) cells and secrete cytokines is blocked which is of advantage for virus infection (Smith and Kotwal, 2002). Perhaps the virus infects the immune cells that play an important role in host defense as e.g. NK cells play an important role in early (first three days) host immune response after poxvirus infection. The latter implies that $\mathrm{T}$ cell responses seems to play a more important role in the early rather than the late phase of primary, acute infections (Gordon et al., 2011; Xu et al., 2004b).

Not only did Sanchez-Puig and colleagues find that VACV WR and MVA showed a preference for certain cell populations (Sanchez-Puig et al., 2004), but this study also showed that the calpox virus seems to have preferences for certain cell populations. This may indicate that factors other than host range genes like the accessibility and the amount of receptors, ability to internalize the virus and the metabolic state of the cell might influence the infection rate of PBMCs and immune cells in the buffy coat.

\subsubsection{Detection method for calpox virus}

The calpox virus was detected in blood by using different methods. The first method was the detection of the calpox virus antigen in buffy coat and PBMCs by flow cytometry, secondly viral DNA was quantified by real-time PCR and the third method was the virus isolation from buffy coat by plaque assay. Comparison of the different calpox virus detection methods revealed that real-time PCR and calpox virus antigen staining by flow cytometry are very sensi- 
tive methods and more sensitive than virus isolation. When the same animals were first tested, calpox virus DNA was detected in only one of three animals at 3 dpi by real-time PCR. Calpox viral antigen was detectable in immune cells of at least two day- 3 animals in $\mathrm{CD}^{+} \mathrm{CD} 20^{+}$ cells by flow cytometry. In animals that had the highest percentages of calpox ${ }^{+}$cells by flow cytometry, the highest viral loads were also detected by real-time PCR. Aside from real-time PCR, calpox virus antigen detection in buffy coat and PBMCs by flow cytometry was the most sensitive method used here.

The real-time PCR and poxvirus antigen staining by flow cytometry methods are also the fastest methods. Since replicating calpox virus detection by plaque assay was first observed at $7 \mathrm{dpi}$ in one of three animals, this seems to be the least sensitive of the tested methods.

Calpox virus antigen staining by flow cytometry has its advantages. It is possible to test different immune cells in one approach and detect the infection of animals supplementing realtime PCR and plaque assay. Because a lower background staining (baseline) was detected in cells from buffy coat of the controls animals compared to purified PBMCs, the calpox virus antigen staining by flow cytometry with cells from buffy coat is prefered.

\subsubsection{Onset of viremia is dose dependent as determined by real-time PCR, plaque assay and calpox viral antigen staining}

Viremia starts at 3 dpi in cases with high dose infection ( $3.5 \times 10^{5}$ pfu calpox virus) as calpox virus antigen positive $\left(\right.$ calpox $\left.^{+}\right)$cells in buffy coat and PBMCs were detected at this time point (\#14484 and \#14991) and calpox virus DNA in blood was detected by real-time PCR at 3 dpi (\#14484). Replicating virus was not detected at 3 dpi.

However, based on the results of calpox DNA copy numbers in different organs from 7 to 12 dpi, it can be concluded that after a local virus replication at the viral application site the viremia starts between 6 and $7 \mathrm{dpi}$ (with the low inoculation dose). In the low-dose group ( $8.3 \mathrm{x}$ $10^{3}$ pfu calpox virus) earliest calpox viral DNA in blood was detected in two of three animals at 7 dpi (\#14966 and \#14483) but not at 5 dpi. At 7 dpi the first replicating virus was also detected in one of three animals (\#15095). Evidence of a viremia that starts between 6 and 7 dpi with the low dose $\left(8.3 \times 10^{3}\right.$ pfu calpox virus), exists and it can therefore be assumed that the onset of viremia is dose dependent.

\subsubsection{1 (II) Early dissemination and pathological sequelae of infection}

It has been reported that once cells became pox-antigen positive and remained positive, the non-human primates (NHPs) developed more severe symptoms and reached moribund end- 
points (Song et al., 2013a). Unfortunately, this was not analyzed here because animals were euthanized at defined time points even though in this study the $\mathrm{CD}^{+} \mathrm{CD} 20^{+}$subset and intermediate $\mathrm{CD} 14^{+} \mathrm{CD} 16^{+}$monocytes were positive for calpox antigen as determined by flow cytometry at 3 dpi. Future studies with the testing for poxvirus antigen positive cells in this model might give insights of a correlation between early positive poxvirus antigen staining, disease progression and lethality since early positive pox staining is also a strong predictor for lethality in the studies of Song and colleagues.

Little was known about the early phase of the calpox virus pathogenesis. To understand the early dissemination and pathological sequelae of calpox virus infection in CM the pathogenesis study was conducted. The pathogenesis study provides evidence that after intranasal calpox virus application the nose represents the portal of viral entry in the calpox/maroset model and therefore early dissemination starts in the nasal mucosa.

Earliest and highest detection of calpox virus DNA and replicating calpox virus was in the nasal mucosa at 3 dpi (with the high inoculation dose). The earliest and highest detection of the calpox virus DNA was also detected in the nasal mucosa at 5 dpi (with the low inoculation dose). Therefore, this location is the portal of viral entry. Other organs (eyes, esophagus, lung, tongue and tonsil) were positive for calpox viral DNA and/or replicating calpox virus at 3 dpi and for calpox DNA copy numbers at 5 dpi (with the low dose) (nasal mucosa, submandibulary LN and tonsil). When analyzing the early dissemination, viremia starts at 3 dpi (with the high inoculation dose) in the calpox/marmoset model after i.n. inoculation. The results outlined in a dissertation conducted in parallel show that the trafficking of infected macrophages presumably lead to a secondary replication in the submandibulary LN and the spleen (Schmitt, 2015).

Virus replicated efficiently in the bone marrow, nasal mucosa and submandibulary LN. Two assays (real-time PCR and endpoint dilution assay) were compared and the organs contained high viral levels as manifested in both tests (between $10^{12}$ and $10^{17}$ calpox DNA copy number/g tissue and between $10^{8}$ and $10^{9} \mathrm{TCID} 50 / \mathrm{g}$ tissue). This also indicates a progressive infection. In comparison to the intravenous (i.v.) inoculation route decribed by Kramski and colleagues, the highest viral loads were measured in skin, spleen, lung, lymph node, tonsils and trachea and indicate lower viral loads when compared to the results of this study (between $10^{7}$ and $10^{8}$ calpox virus genome equivalents (GE)) (Kramski et al., 2010).

The data suggests that the calpox virus initially replicated in the upper respiratory tract followed by the systemic spread. Calpox virus probably disseminated through the blood stream (viremia; see 4.1.6). Onset of viremia is dose dependent as determined by real-time PCR, 
plaque assay and VACV antigen staining. It is probable that the calpox virus first replicated in the nasal mucosa and then spread from the nasal mucosa to the surrounding lymphatic tissue (submandibulary LN and tonsil) where it leads to secondary replication. The virus probably spreads from there to other organs of the respiratory tract and other surrounding tissues (eyes, esophagus, lung and tongue) by viremia followed by diffuse dissemination in other organs. Low level infection as indicated by the results from both assays (real-time PCR and endpoint dilution assay) were determined in the central nervous system (CNS), parotid gland, small intestine, stomach, esophagus, parotid gland and vagina/testicle suggesting that the calpox virus replicated less efficiently in these organs. These findings are consistent with other studies (Tucker, 2011).

The pathogenesis in this model is comparable to that of smallpox infection, because it also starts in the respiratory mucosa, spreads to the lymph nodes and is followed by viremia. The same process was observed in a study where cynomolgus macaques were infected with aerosolized MPXV by using a head-only exposure chamber with a collision nebulizer (Zaucha et al., 2001).

As in this study, the virus was detected in various organs in lethal human MPXV and VARV infections. Kramski and colleagues described the involvement of organs of the digestive tract in the calpox virus/marmoset model opposed to human VARV infections (Fenner et al., 1988c), which was verified in this study. A monocytic cell associated viremia starts at 7 dpi and causes systemic dissemination of the virus. The virus is detectable in a broad spectrum of organs, mainly in epithelial cells and macrophages, but also in endothelial cells. The viremia proceeds in viral replication and typical calpox lesions in lung, liver, lymphatics, adrenal gland, mucocutaneous junctions, and skin as described in a parallel dissertation (Schmitt, 2015).

Because $12 \mathrm{dpi}$ is already close to the moribund endpoint, the highest $\mathrm{TCID}_{50} / \mathrm{g}$ tissue and calpox DNA copy number/g tissue were measured in 12 dpi animals. In late state infection (12 dpi), multiple immune cell subsets were also positive for calpox virus antigen. The wide cell tropism of the calpox virus is demonstrated in a study of Kramski and colleagues. It has been shown that independent of the infection route and inoculated viral dose, high copy numbers of virus genomic DNA and virus-specific mRNA indicating active replication were found in a broad spectrum of organs and tissues (Kramski et al., 2010). In lethal human monkeypox virus (MPXV) and variola virus (VARV) infections, multiple organs are also generally involved and the virus was also found in various organs (Fenner et al., 1988b). It has been 
described that a systemic macrophage response is required to contain a peripheral poxvirus infection (Davies and Parekh, 2017).

It has already been shown for VARV infections in cynomolgus macaques that the virus replication was probably associated with organ dysfunction and multisystem failure of all the organs (Jahrling et al., 2004), which assumably was also the case in this study. In the calpox virus/marmoset model, all animals that developed clinical symptoms died. In natural VARV and MPXV infections, only part of the diseased patients had a lethal outcome. Seroconversion was not observed in animals that died within 12 days and was not analyzed in the pathogenesis study. Obviously, the survival time of the CM after infection is too short to detect titres of virus-specific IgM antibodies at the time point of death. It has also been reported that intranasal infection of the calpox virus probably resulted in an overwhelming infection and death occurred before the humoral immune system could respond by developing IgG and neutralizing antibodies (Kramski et al., 2010).

In the calpox virus/marmoset model, most of the clinical signs resemble those commonly found in smallpox. In this model, however a drastically shorter time was reported between the first appearance of clinical signs only one or two days before death (Kramski et al., 2010; Schmitt, 2015). In human smallpox and MPXV infection a longer period of two to five days were reported where fever, malaise, prostration, headache and backache occurred which were followed by the development of a maculopapular rash. The first symptoms rapidly developed into more severe clinical findings in the upper respiratory tract and the lymphatic system as well as hemorrhagic edema. As reported in a dissertation conducted in parallel (Schmitt, 2015), animals that were euthanized or died unexpectedly between 12 or $16 \mathrm{dpi}$, showed varying symptoms. Two animals from the group that was euthanized at $12 \mathrm{dpi}$ had only a very few typical pox lesions on the skin, mucocutaneous junctions and mild nasal discharge. The animals with symptoms showed signs of fatigue, anorexia, moderate to severe seromucous nasal discharge and multiple pox lesions on skin and mucocutaneous junctions one to two days prior to death. Another difference to MPXV and VARV patients is that those present with a more or less confluent rash (Fenner et al., 1988a; Fenner et al., 1988c; Jezek et al., 1987; Parker et al., 2007). Only sporadic and small skin lesions distributed on the face, abdomen, and thighs after i.n. infection with calpox virus occurred in CM (Kramski et al., 2010). Studies have already shown that even with a high calpox virus dose it was impossible to induce an infection that led to skin lesions that were distributed confluently on the body of CM that is characteristic and previously demonstrated in macaques infected with MPXV and VARV (Hooper et al., 2004; Jahrling et al., 2004; Jezek et al., 1988; Stittelaar et al., 2005). 
The morphology of the skin lesions differs between calpox virus and VARV or MPXV lesions. The calpox virus induced skin lesions were flat and small in size (2-5 $\mathrm{mm}$ in diameter) whereas the lesions induced by VARV or MPXV, reach a diameter of up to $10 \mathrm{~mm}$, but the size of the animal might also play a role (Kramski et al., 2010). The severe lymphadenopathy was found in calpox virus infection and occurs as a classic alteration in many OPXV infections. The lymphadenopathy is comparable to the clinical picture of the cowpox virus (CPXV) and MPXV infections in humans and also NHP models but not in human smallpox (Fenner et al., 1988a; Heraud et al., 2006; Hooper et al., 2004; Kramski et al., 2010; Sale et al., 2006).

\subsection{Vaccination studies}

\subsubsection{Vaccine efficacy}

In addition to efficacy testing of next-generation vaccines against orthopoxviruses in mouse models, further proof has to be provided by using suitable large animal models. In this respect animal models with an immune system very similar to the human one are preferable. This is the reason why non-human primates were chosen as large animals for such studies. To this end, efficacy of candidate vaccines has been investigated in macaques which were challenged with MXPV or VARV. However, mostly monkeys were exposed to high viral doses by the intravenous route, thus not representing the natural transmission route of the two humanvirulent viruses. Since in the calpox virus/marmoset model very low viral doses administered intranasally are sufficient for infection and rapidly lead to death of all animals, this model can provide meaningful efficacy data through a natural transmission setting in a short period of time. The primary read-outs for protection are viral loads and survival.

Earlier studies in the calpox virus/marmoset model have shown that after a waiting period of ten weeks between single vaccination with vaccinia virus Lister Elstree (VACV LE) intradermally (i.d.) and intranasal calpox challenge all vaccinees were protected. When vaccinees were already challenged four weeks after VACV LE was given, only one of four was protected (Kramski, 2009). The conventional VACV vaccine against smallpox is known to be associated with considerable side effects, particularly in older or immunocompromised vaccinees (Mota et al., 2011). Thus, in this study the differently attenuated, safer VACV vaccines MVTT and MVA were evaluated in CM for their efficacy against intransal low-dose calpox virus exposure after a 4- (study I) or 10-week waiting period (study II) between last immunization and viral challenge. To compensate for their reduced replication capacity compared to replication-competent VACV LE, both vaccines were given twice four weeks apart as was 
reported for MVA before (Stittelaar et al., 2005). Because MVTT was highly immunogenic in mice after noninvasive mucosal administration, either through the intransal (Yu et al. 2009) or peroral route (Huang et al. 2009), the same routes were chosen for immunization of CM with MVTT. As numbers of monkeys were limited not allowing to test both mucosal routes combined with the different waiting periods, relying on efficacy data in the mouse model (Yu et al., 2010) in vaccine study I only the efficacy of intranasal MVTT applicaton was investigated. By contrast, intranasal and peroral immunizations were compared side by side, when a longer waiting period (10 weeks) should allow for prolonged maturation of vaccine-induced immune responses and thus for better protective effects.

When the time period until the animals died (days post challenge) is compared between the vaccine failures and the mock immunized control animals a significant difference was detetcted $(* * * ; p=0.0002)$. Therefore, a vaccination effect is present in the vaccine failures, but this effect can not be explained by the data raised in this thesis.

Overall, independently of the waiting period immunization with MVTT protected $67 \%$ of the animals whereas immunization with MVA was only protective in $13 \%$ of the test animals.

While MVA was not protective when last immunization and challenge were spaced four week apart, intranasal immunization with MVTT protected three out of four monkeys after this waiting period, thereby confirming MVTT efficacy findings in the mouse model (Yu et al., 2010). The longer waiting period seemed to slightly improve protection in the MVA immunized group as indicated by survival of one of four vaccinees, whereas in animals immunized with MVTT i.n. no increased protection rate was observed. Similar protective rates were observed after peroral MVTT immunization. Thus, MVTT immunization by the two different mucosal routes seemed to provide similar protection levels, which however did not reach those achieved by the fully replication-competent VACV LE. The high efficacy of VACV LE still represents the gold standard for a prophylaxis against orthopoxviruses. This high level of protection could not be achieved by the more attenuated VACV vaccines, but considering the potential side effects of the conventional smallpox vaccine, MVTT seems to be a safe and well-tolerated smallpox vaccine candidate for humans. Compared to MVTT, MVA-mediated protection was poor in the calpox virus/marmoset model. This might be attributable to the stringency of the calpox virus challenge. If calpox virus replicates to a certain threshold, the virus is always lethal for CM. This is in contrast to the MPXV/macaque model in which dosedependent stages of infection occur. In that model MVA mediated protection from disease, but not from infection (Earl et al., 2004; Stittelaar et al., 2005). Because of the reduced replication competencies of MVA and MVTT (MVA < MVTT) in contrast to the fully replication 
competent VACV LE development of protective immune responses through these vaccines may require even a longer period of time as compared to VACV LE. Still, MVTT- or MVA induced immune reactions may never be sufficient for complete protection in the calpox virus/marmoset model. Interestingly, and controversial to the own calpox challenge data, already four days after a single MVA immunization macaques were protected from pathogenic sequelae of a MPXV challenge, most likely due to rapidly developing antibody and $\mathrm{CD}^{+} \mathrm{T}$ cell responses (Earl et al., 2008).

Kinetics of calpox viral DNA load in blood from all vaccine failures were comparable to those observed in mock immunized animals. Moreover, in study II the data on replicating virus confirmed those on viral DNA.

In three protected MVTT-vaccinees of study I very low levels of calpox virus DNA were transiently observed starting on day 14 or 21 . Whether this also translated into infectious virus, could not be assessed. In study II two protected vaccinees exhibited those viral blips as well, but remained negative for infectious virus. Thus, albeit calpox virus probably replicated in some protected animals, the immune responses were obviously able to control viral dissemination and to finally eliminate the virus. Therefore, these animals were protected against calpox virus-induced clinical manifestations and overall are regarded as protected. Vaccinees were considered being protected according to the criteria survival and viral load.

Low-level viral DNA blips in blood of protected vaccinees were detected. It seems as when the calpox DNA copy numbers increase upon a certain threshold (between $10^{3}$ and $10^{4}$ ) animals die from disease.

\subsubsection{The role of the humoral immune response in the calpox virus/marmoset model}

In this study the role of humoral serum responses was analyzed by the analysis of binding antibodies by enzyme-linked immunosorbent assay (ELISA), mapping antibodies against linear B cell epitopes and the investigation of neutralizing antibody titres. In non-human primates as well as mouse models, no protection was observed before antibodies were developed (within a period of seven to ten days after vaccination) (Staib et al., 2006; Stittelaar et al., 2006).

In this system it was not possible to test the role of the complement due to the limited availability of plasma. Plasma samples were only tested without heat inactivation because of the type of sample used here. 


\section{Binding antibodies by enzyme-linked immunosorbent assay (ELISA)}

A correlation between the presence of serum antibodies and protection has been reported for human orthopoxvirus (OPXV) infections (Downie and Mc, 1958; Mack et al., 1972; Sarkar et $a l ., 1975)$. In the vaccination studies presented here the antibody responses determined in the day-of-challenge plasma were not associated with protection.

The reasons for this are not clear, but should not be a matter of immune response maturation. In this respect it has been reported that in macaques MVA immunization led to a more rapid development of cellular and humoral immune responses compared to Dryvax immunization and was already $100 \%$ protective against a lethal MPXV dose ten days after vaccination (Earl et al., 2008). Furthermore, those reactions were stable and very similar in the two vaccine groups four weeks after vaccination. Thus, such stability of immune responses is also to be expected in the marmorset vaccinees, and lack of an immune correlation cannot be explained by waning immunity. It should be noted that ELISA antibody levels against calpox virus (expressed as $\mathrm{OD}_{450 \mathrm{~nm}}$ ) were considerably lower than against VACV which might me attributable to lower affinity of antibodies towards calpox viral particles.

Interestingly, independent of the vaccine variant and the immunization routes used an ananmnestic antibody response was not only noticed in all MVA and MVTT vaccine failures, but also in $75 \%$ of the protected MVTT vaccinates. This indicates that low-level calpox virus replication must have occurred in those protected animals displaying the stark rise in antibody levels after challenge, presumably at the vaccination site, however, not leading to viremia.

When considering all protected animals, two MVTT and one MVA vaccinate lacked this response which suggests sterile immunity.

\section{Specificity of antibodies against B cell linear epitopes in relation to challenge outcome}

Since binding antibody levels against whole orthopoxvirus antigen did not discriminate between protected and unprotected vaccines, antibodies were analyzed at a high resolution level by means of peptide microarrays. This technique allows to measure antibodies against linear epitopes of an antigen, in this case against vaccinia virus proteins. For the generation of overlapping peptide libraries, the immunodominant VACV WR proteins A27, A33, B5, D8, H3, and L1 (Matho et al., 2012) were chosen. They represent surface proteins and are targets of neutralizing and protective antibodies (Moss 2011).

The proteins A27, D8, H3 and L1 are involved in virus entry and/or fusion (Moss, 2011), whereas the proteins A33 and B5 play a role in virus spread (Moss, 2011). 
A27 is an enveloped protein and is anchored to the viral membrane of the MV and one of the three glycosaminoglycan adhesion molecules. Linear epitopes in A27 have already been described as targets of protective antibodies induced by vaccination against smallpox (Kaever et al., 2016).

A33 (Roper et al., 1996) and B5 (Engelstad et al., 1992; Isaacs et al., 1992) are transmembrane proteins that are exposed on the outer surface of the enveloped virion (EV), but are not found on the intracellular mature virions (IMV) (Chan and Ward, 2010). These proteins were chosen, because deletion of their genes resulted in decreased virus spread (Moss, 2011). A33 and B5 have also been shown to be targets of comet-reducing antibodies. A33 and B5 are EV membrane proteins and were protective in the intranasal VACV and ectromelia virus (ECTV) footpad infection mouse models (Fang and Sigal, 2006; Fogg et al., 2004; Galmiche et al., 1999).

D8 is another membrane protein, which was chosen because the mAb LA5 binds to the VACV envelope protein D8 and is able to neutralize VACV when complement is present (Matho et al., 2012). D8 binds to the chondroitin sulfate on the host cell surface, but not to heparan sulfate like proteins H3 and A27 (Matho et al., 2012).

$\mathrm{H} 3$ is, like A27, involved in cell adhesion of the MV to glycosaminoglycan heparan sulfate of the host cell (Matho et al., 2012).

$\mathrm{L} 1$ is a capsid protein of the MV, which is located on the mature virion (MV) and functions as an entry/fusion protein. L1 is likely associated with the neutralization of VACV (Kaever et al., 2016). Furthermore, antibodies against A33, B5 and L1 were able to protect mice against lethal challenge with VACV (Fogg et al., 2004).

In this study, unique antibodies against linear B cell epitopes in proteins L1, A33, and B5 were only identified in protected animals almost exclusively after (epitopes 1-4), but in one case also before challenge (epitope 5). The failure to detect a specific reaction pattern of antibodies directed against distinct amino acid sequences might be explained by (i) low antibody levels which were not sufficient to result in a reaction in the peptide array. Another reason for not detecting antibodies against B cell epitopes that correlate with protection might be that (ii) the sequences of the printed VACV WR peptides are too divergent to those of the vaccines (MVA and MVTT) thereby missing antibody responses. Alternatively, (iii) at least antibodies against epitopes 1-4 developed de novo post challenge. The theory behind this is that other antibodies may play a role in the exposure of these four epitopes to the immune system upon binding to the challenge virus thereby unmasking usually unaccessible regions. This phenomenon was observed (a change in the 3D structure) in the presentation of an important neutral- 
izing epitope in the haemagglutinin of the influenza virus upon binding of antibody to different regions (Leikina et al., 2002). Finally, another plausible argument for lack of antibodies against linear amino acid sequences could be that (iv) almost all generated antibodies were directed against conformational or discontinuous epitopes which prevents any detection in the peptide microarray.

A further reason why protective antibodies might not have been screened by peptide microarray at challenge could be that the proteins that were synthetized and printed on the peptide microarrays did not include the relevant sequence.

Also of note is that after the longer waiting period between last immunization and challenge in study II, the total number of identified epitopes $(n=12)$ was higher than in study $\mathrm{I}(\mathrm{n}=4)$ suggesting maturation of the humoral response over time.

Despite the uniqueness of the development of antibodies against some epitopes after challenge (Table 3-3), because of the timing those responses could not have contributed to protection from the calpox challenge.

\section{Detection of neutralizing antibody titres}

Neutralizing antibodies play an important role in the protection against poxviruses (Gilchuk et al., 2016b; Moss, 2011). Since the level of neutralizing activity in immune serum in thought to be the best laboratory predictor of protective immunity to OPXV infections in humans (Mack et al., 1972), I analyzed the neutralizing antibody titres in this study.

Assumably, vaccination with MVTT p.o. induced more neutralization than MVA i.m. Levels of binding antibodies and neutralizing antibody titres were lower against the calpox virus than the immunogen VACV LE.

In vaccination study II the mean value of the MVTT p.o. immunized group titres was higher than MVTT i.n. followed by MVA i.m. immunized group titres when the serum samples were tested against VACV LE and calpox virus. One can assume that MVTT p.o. induced more neutralizing antibodies than MVTT i.n. and MVA i.m.

There were no neutralizing antibody titres detected in some animals that were protected, e.g. \#14414. Previous studies have shown that immunological protection can also be provided by preventing the release of virions rather than by neutralization (Vanderplasschen and Smith, 1997) of the EEV particles. In that case antibodies against EEV particles can cause the aggregation of EEV particles on the cell surface (Ichihashi, 1996).

To sum up, there was no correlation between protected and unprotected animals in the development of neutralizing antibodies as well as the other tests like B cell epitope fine mapping by 
peptide microarray and ELISA when analyzing the humoral response at challenge. When analyzing the humoral response, it can be assumed that MVTT p.o. induced more neutralizing antibodies than MVTT i.n. and MVA i.m.. In peptide microarray, antibodies against different epitope regions that are exclusive in protected animals were detected at different time points after challenge (Table 3-1). There was no correlation between the level of binding antibody responses in protected and unprotected animals detected (ELISA).

\subsubsection{The role of the cellular immune response in the calpox virus/marmoset model} Polychromatic flow cytometric staining of differently fluorescently marked cells in whole blood

Significant differences in $\mathrm{T}$ and $\mathrm{B}$ cell numbers, their expression of activation markers CD69 as well as HLA-DR and CD80, were neither observed between the different vaccine groups nor between protected and unprotected animals on the day of the challenge. This suggests that the different vaccination strategies did not affect innate and adaptive immune cell numbers. There was also no correlation between cell numbers of protected and unprotected animals at challenge (data not shown). Furthermore, the kinetics of the tested cell subset frequencies (e.g. T and B cell frequencies) were similar and no differences between vaccination groups were detected. Although cell numbers did not change and frequencies were similar as well, it is possible that differences in the vaccine-induced secretion of cytokines occur that might play an important role in the protection of $\mathrm{CM}$ in the calpox virus/marmoset model. To my knowledge, there are no reports on phenotyping of immune cells after poxvirus infection for CM. Mucker and colleagues (Mucker et al., 2015) infected CM with MPXV, but no phenotyping of immune cells was done after the infection. Song and colleagues found that a monkeypox virus (MPXV) infection of rhesus macaques led to expanded frequencies and absolute numbers of NK cells in blood (Song et al., 2013b). In the calpox virus/marmoset model, this effect was not detected by flow cytometric staining. Unfortunately, earlier studies in the calpox virus/marmoset model with VACV LE BN vaccination did not include analyses of the vaccine induced cellular response.

In the vaccination studies, cell numbers of $\mathrm{CD} 20^{+} \mathrm{B}$ cells, $\mathrm{CD} 3^{+}, \mathrm{CD}^{+}$and $\mathrm{CD} 8^{+} \mathrm{T}$ cells, $\mathrm{CD} 4^{+} \mathrm{CD}^{+} \mathrm{T}$ cells, CD14 ${ }^{+}$cells (monocytes), NK cells, mDCs, pDCs, CD16 ${ }^{+} \mathrm{CD}^{+} 6^{+}$cells, $\mathrm{CD} 14^{++} \mathrm{CD} 86^{+}$cells increased shortly before the animals became moribund. Also, increases in the expression of the activation marker CD86 in $\mathrm{CD} 14^{+} \mathrm{CD} 16^{+}$cells were observed. This 
strong increase of the absolute cell numbers shortly before death may be due to a possible cytokine storm for which, however, no evidence exists.

\section{Proliferation of $\mathrm{CD}^{+}, \mathrm{CD}^{+} \mathrm{CD}^{+}$and $\mathrm{CD}^{+} \mathrm{CD}^{+} \mathrm{T}$ cells}

Proliferation of $\mathrm{CD}^{+}, \mathrm{CD}^{+} \mathrm{CD}^{+}$and $\mathrm{CD}^{+} \mathrm{CD}^{+} \mathrm{T}$ cells upon stimulation with VACV (here MVA) was analyzed and at no measured time point were significant differences between the vaccination groups detected. Therefore, the different vaccines did not differ in their proliferating activities of $\mathrm{T}$ cells.

Moise and colleagues evaluated a $\mathrm{T}$ cell epitope vaccine based on vaccinia/variola sequences and induced protective immunity against VACV infection in mice by $\mathrm{T}$ cell responses alone and without the necessity of B cell priming (Moise et al., 2011). In contrast, the proliferative activity of $\mathrm{CD}^{+} \mathrm{CD}^{+} \mathrm{CD}^{+}$cells was present even though animals died (e.g. \#15100). The proliferative activity was not associated with the protection of the animals from the calpox virus challenge. It needs to be clarified what exact role the $\mathrm{T}$ cell proliferation plays in protection.

\section{3 (III) Identification of immune correlates}

Little is known about the immune response in CM after a poxvirus infection (Kramski et al., 2010). Data from two vaccination studies were merged to identify any immune correlates of protection. So far, it is still unclear why the vaccine failures died. These animals all developed high viral load viremia and in case where analysis was possible, also moderate to high titres of infectious virus in whole blood when compared to unvaccinated control animals. Lower levels of infectious virus shortly before or at death were observed in MVTT vaccine failures independent of the vaccination route when compared to those not protected by MVA immunization.

This suggests that calpox virus replication was slightly reduced in MVTT vaccine breakthroughs which, however, did not prevent the animals from becoming moribund. A correlation between the presence of serum antibodies and protection from death has already been reported for human orthopoxvirus (OPXV) infections (Downie and Mc, 1958; Mack et al., 1972; Sarkar et al., 1975). There is, however, no relation between the levels of ELISA antibody responses and protection. If neutralizing antibodies (nAbs) are present, protection does not depend on a high level of antibody response in ELISA because animals were protected also with a low titre (e.g. \#14478) and vice versa (e.g. \#14603). No association was observed 
between the breadth of high resolution antibody responses as determined by peptide microarray and protection. Nevertheless, neutralizing antibodies seem to play an important role since B cell epitope fine mapping by peptide microarray revealed antibodies against epitope regions that are exclusicely present in protected animals at different time points post challenge. Neutralizing antibodies were determined, because it has been reported that those play an important role in the protection against poxviruses (Edghill-Smith et al., 2005). Data from this study suggest that MVTT was a better inducer for neutralizing antibodies than MVA. Poorer induction of neutralizing antibodies through MVA compared to Dryvax immunization has also been reported for macaques (Earl 2008). Also, there was no correlation between titres of neutralizing antibodies determined in plasma from the day of challenge and protection. Indeed, the MVTT vaccine failures developed moderate neutralizing antibody titres (e.g. \#14169) suggesting that the presence of these functional antibodies did not influence the disease course. The phentopyping of immune cells was analyzed, but since no significant differences between groups were detected in the analyzed T- and B cells and activation markers at the day of challenge, the different vaccination strategies did not have an effect on the different cell numbers at challenge. The $\mathrm{T}$ cell proliferation assay also revealed no significant differences between the vaccine groups at the time points analysed. The proliferative activity was also not associated with the protection of the animals from calpox virus challenge.

It is tempting to speculate that death of calpox-infected animals resulted from a collapse of the immune system and organ dysfunction. With the findings of a dissertation conducted in parallel (Schmitt, 2015), it seems to be reasonable that the deaths are related to multi-organ system failure, immune system compromise and resulting opportunistic bacterial infections with sepsis. Another explanation for the death of common CM (CM) might be a cytokine storm, since in human hemorrhagic smallpox the cause of death was often suggested as toxemia or a cytokine storm (Councilman et al., 1904; Fenner et al., 1988b; Jahrling et al., 2004; Johnson et al., 2011; Martin, 2002; Ricketts, 1908). A cytokine storm is also assumed to play an important role in a MPXV model in cynomolgus macaques (Macaca fascicularis). Strongly elevated levels of certain cytokines (CCL2, IL-6, IL-8, IFN- $\gamma$ ) were detected in cynomolgus macaques following intravenous infection with VARV and cowpox virus (Jahrling et al., 2004; Johnson et al., 2011). The cytokines that seem to play an important role in poxvirus immune response were not analyzed here. Due to the limited availability of reagents for CM, details of the immunology of calpox virus pathogenesis have not been studied and are difficult to elucidate.

In the studies of Zielinski and colleagues, cynomologous macaques were immunized with the 
cell culture grown ACAM 2000 vaccine (Wyeth strain) Wyeth-IL15, the genome of Wyeth strain of VACV with the integrated human cytokine IL15, a cytokine with pleiotropic immune modulatory activities (Zielinski et al., 2010). Three years after immunization with that vaccine animals were challenged with a lethal dose of MPXV. Following challenge most vaccinates presented with milder clinical manifestations and complete recovery. This study showed that the immunization with Wyeth-IL15 confered long term protection. IL-15 is essential in the functioning and differentiation of NK cells. Because of the positive effect of vaccination with Wyeth-IL15 as well as the clearance of VACV it contributes to the control (Hugin et al., 1993). Other cytokines (e.g. IL-18) were also detected in cowpox viruses (Smith et al., 2000) as well as cytopkine receptor homologs (e.g. vTNFR, vIFN $\gamma \mathrm{R}$, vIL-1R) (Alcami and Smith, 1992; Loparev et al., 1998; Smith and McFadden, 2002).

In this study, no distinct immune correlates were identified. Poxviruses are able to defend themselves through encoded proteins that are involved in immune evasion and immune modulation (Smith and Kotwal, 2002). Such proteins are able to block several strategies of the host defense, e.g. blocking the activity of many chemokines, cytokines, serine proteases and even complement.

\subsubsection{Advantages and disadvantages of the calpox virus/marmoset model}

When selecting an animal model for viral pathogenesis or prophylactic studies, different aspects (e.g. applicability, costs, ethics) must be considered. The advantages and disadvantages of different OPXV NHP models have been discussed in Schmitt and colleagues (Schmitt et al., 2014).

One disadvantage of the calpox virus/marmoset model is the relatively high maintenance costs compared to rodents or other non-rodent species (Jagessar et al., 2013). For ethical reasons, using this model has the disadvantage that the common marmoset (CM) is closer related to humans than rodents. The small size of the CM also plays a role: animal experimentation and sampling is more difficult to perform in small animals and also the blood volume which can be collected is limited. Another disadvantage of this model is the absence of disease stages as observed for smallpox in humans and MPXV infection of macaques (Schmitt et al., 2014). Although calpox virus belongs to the specific of cowpox viruses and therefore is potentially infectious for humans, epidemiological investigations demonstrated no evidence of transmission to man because persons being in close contact to calpox virus infected and diseased CM were all seronegative for OPXV antibodies (Mätz-Rensing et al., 2012). Still, infectivity in general cannot be excluded (Dr. Stahl-Hennig, personal communication) (Schmitt 
et al., 2014). Furthermore, in non-human primate models there are less animals available than in smaller animal models (Schmitt, 2015). Also, the availability of some critical immunological tools is still very limited for CM.

In general, non-human primates including cynomolgus macaques, rhesus macaques, African green monkeys and CM are the gold-standard for pathogenesis studies and the evaluation of new medical countermeasures, because they fulfill the criteria of the FDA Animal Rule for the highly pathogenic viruses (Safronetz et al., 2013). Advantages of the CM compared to other NHPs are that they are not endangered, are inexpensive to keep and easy to handle (Kramski et al., 2010). Their reproductive rate in captivity is high and they are also available at a reasonable price. CPXV was reported as suitable to study human smallpox, because it shares 19 immunomodulatory genes with VARV, the smallpox agent (Johnson et al., 2011; Seet et al., 2003). Furthermore, the disease in the calpox virus/marmoset model is comparable to human smallpox. The incubation period is similar to that of human smallpox. Another advantage of the calpox virus/marmoset model is that infected animals can be kept at biosafety level (BSL) -2 whereas MPXV must be handeled at BSL-3, and VARV even at BSL-4. Experiments with this virus can therefore be conducted by a larger number of laboratories (Mätz-Rensing et al., 2012). Also, the CMs develop a reproducible lethal, systemic disease with cutaneous pox lesions and die upon low-dose intranasal inoculation within 12-16 dpi (Kramski et al., 2010). Also, a very low infectious viral dose is sufficient to establish infection. The i.n. calpox virus application was established in CM because VARV infection in humans normally occurs via airborne transmission. By infecting the upper respiratory tract, the major natural route of the human VARV infection is mimicked. This inoculation route has been described as advantage to the non-physiological i.v. route because that one "bypasses the mucosa and circumvents a primary replication of the virus in regional lymph nodes and the successive systemic spread via the lymphatic system, altogether representing the first viremia" (Kramski et al., 2010).

This is why the calpox virus/marmoset model is a suitable animal model for the validation of vaccines and antiviral drugs, but also for studying OPXV pathogenesis.

\subsubsection{Relevance of modified vaccinia virus Ankara (MVA) and modified vaccinia virus Tiantan (MVTT) in developing a safer, but efficacious smallpox vac- cine}

The studies of Chen and colleagues suggest that MVTT is a safe and noninvasive smallpox vaccine candidate which induces neutralizing antibodies upon i.n. immunization ( $\mathrm{Yu}$ et al., 
2010). MVTT replicates in many mammalian cell lines, but replication in the brain of mice is lacking underlining the safety of this vaccine (Yu et al., 2010). Mucosal immunization with MVTT protected mice from VACV Western Reserve (WR) challenge. Accordingsly, MVTT is not only a potentially safe and noninvasive smallpox vaccine, but also effective. The efficacy in CM remained to be tested in these studies. MVTT protected $67 \%$ of the animals whilst MVA protected only $13 \%$ of the vaccinees independently of the waiting period. Thus, MVTT seems to provide better protection, but because of small animal numbers per group careful interpretation is required. When comparing all MVTT-induced immune responses with those mounted after MVA, MVTT appeared to be more immunogenic than the highly attenuated MVA and thus looks like a promising new smallpox vaccine candidate. Yet, it is unclear why lethal vaccine breakthroughs occurred in some MVTT vaccinated animals. One explanation was previously given (Schmitt, 2015). The interaction of intranasally applied MVTT with the NALT could stimulate a local respiratory mucosal immunity (Xu et al., 2014). Cytotoxic Tlymphocytes that recognize the intranasally calpox virus challenge could have evoked excessive immune responses that resulted in severe pneumonia (Schmitt, 2015). This could have held true for MVTT vaccine failure. As MVA breakthroughs shared the same pathological alterations, local expansion of challenge virus at the portal of entry might have attracted systemic virus-specific CTLs leading to a similar burst of immune reaction.

This study could not clarify whether the mucosal application of MVTT led to a cytotoxic T cell response. Besides the fact that the virus-specific proliferative activity of the $\mathrm{CD}^{+} \mathrm{CD}^{+} \mathrm{CD}^{+}$subset was slightly higher in MVTT vaccines compared to that of MVA ones, there were no hints of a cytotoxic $\mathrm{T}$ cell response that may be the cause for the severe pulmonary lesions as described in a parallel dissertation (Schmitt et al., 2017). Another explanation for the pulmonary changes might be a cytokine storm, which can occur independently of the vaccination route. When a pathogen enters a susceptible host, the immune sytem fights against it, different cytokines signal the immune cells (e.g. T cells, macrophages) and then they migrate to the infection site.

\subsubsection{The role of the different vaccination routes}

Mucosal vaccination with VTT in mice (i.n. or oral) induced higher sustained levels of neutralizing antibodies (nAbs) than i.m. and subcutaneous vaccination (Lu et al., 2011). Therefore, the intranasal and peroral routes were tested here. MVTT was developed from the Asian Tiantan strain by removing the hemagglutinin gene and its mucosal application protected mice against the VACV Western Reserve (WR) strain (Yu et al., 2010). Mucosal immuniza- 
tion seems to be a successful application route using this vaccine. In comparison to systemic vaccine application, the mucosal application has the advantage that it triggers local (in the NALT) as well as systemic humoral and cellular immune responses (Holmgren and Czerkinsky, 2005). In addition, a better compliance with this noninvasive vaccination is expected. Technically, the application is easy and can be done by untrained personnel. The mucosal application is also of advantage, because no injection needles are required thus preventing the risk of iatronic transmission of blood-borne infections (Lycke, 2012). A disadvantage is that the mucosally applied vaccine might be swallowed or eliminated through sneezing. Therefore, it would not be clear as to how much of the applied dose remains in the organism and whether there is enough time for antigen adsorption before mucociliary clearance (Velasquez et al., 2011). Therefore, in a future approach systemic vaccination of CM with MVTT should be analyzed. It would be interesting to know if the same dose given intranasally confers a higher level of protection. Huang and colleagues showed in a mouse model that mucosal application of MVTT led to higher levels of nAbs than an intramuscular injection (Huang et al., 2009), but the results in a monkey model might be different.

Nevertheless, distinguishing between different mucosal application routes (i.n. or p.o.) in MVTT immunized animals in this study does not seem to be of importance when considering the humoral responses. Neutralising antoibody titres did not vary significantly between the MVTT p.o. group and the MVTT i.n. group. These data are in accordance with findings by Huang and colleagues in mice (Huang et al., 2009). MVA was chosen because of its high attenuation resulting in lack of replication in most mammalian cell lines. Thus, it was important that all MVA particles were contained in the body through injection.

The mucosal vaccination route does not seem to have an influence on the ELISA antibody titres because no significant difference in titres between the vaccine groups was observed. B cell epitope fine mapping resulted in the detection of antibodies against different epitope regions that were exclusively found in protected animals with no notable difference in MVTT i.n. and MVTT p.o. immunized animals. Antibodies against epitope 2 were only discovered in one MVTT p.o. animal, but antibodies against the other epitopes appeared in both MVTT immunized groups. Therefore, mucosal immunization with MVTT is advantageous for many reasons, but the route seems to be less relevant.

\subsection{Conclusion and outlook}

From the findings of this thesis it can be concluded that the first replication of the calpox virus is located at the inoculation site, i.e. the nasal mucosa. It can be postulated that the onset 
of dissemination of the virus via blood (viremia) (DNA copies in blood) is dose dependent and starts already at $3 \mathrm{dpi}$ when a high inoculation dose is used and between 6 and 7 dpi when a low inoculation dose is administered. The data suggests that the calpox virus initially replicated in the upper respiratory tract, most likely in the nasal mucosa, next in the surrounding lymphatic tissue (submandibular LN and tonsil) and finally spread systemically through viremia. When a high dose of calpox virus is applied intranasally, a not quantifiable proportion of virus is probably swallowed, thereby leading to infection of tissues located more distant from the viral entry (tongue and esophagus). In the prefinal phase, all organs became infected. MVA did not provide solid protection to CM in this model. In this approach, the overall highest protection was mediated by MVTT independent of the vaccination route and waiting period. When comparing the serological findings in MVTT and MVA vaccinees, MVTT seemed to be more immunogenetic in this model. Accordingly, the mucosal needle-free application of MVTT appears to be a promising smallpox vaccine candidate. Little is known about the immune response in $\mathrm{CM}$ after a poxvirus infection and unfortunately, no obvious immune correlates of protection were found in this study.

Details of the immunology of calpox virus pathogenesis (e.g. the role of cytokines in the calpox virus/marmoset model) have not been studied so far, and due to the limited availability of reagents for common $\mathrm{CM}(\mathrm{CM})$, they are difficult to elucidate. This remains to be studied in the future.

According to Zielinski and colleagues (Zielinski et al., 2010) studies with human cytokines integrated into VACV genome represent an interesting approach which is worthwhile to be investigated in the calpox virus/marmoset model.

In further studies, recombinant proteins that are known to be determinants for the induction of protective and neutralizing antibody could be used for seroanalysis in order to ultimately discover immune correlates that could help to inform smallpox vaccine development. New studies could also show if efficacy of MVTT immunization is increased through systemic vaccination. Moreover, it would be interesting to test the efficacy of a further third generation live-attenuated smallpox vaccine (e.g. the Japanese strain LC16m8) and how it compares to MVTT and MVA in the calpox virus/marmoset model. 


\section{SUMMARY}

\section{Li Lin Gan (2017)}

Smallpox was successfully eradicated in 1980 and mass vaccination stopped between 1976 and 1984 in Europe. Herd immunity has been waning since then and left the population unprotected against orthopoxvirus (OPXV) infections. In the light of bioterrorism and emerging zoonotic diseases an increasing interest in the development of antiviral drugs and safer vaccines against OPXVs arises. A non-human primate model was established in cooperation with the Robert Koch Institute (RKI). Common CM (CM, Callithrix jacchus) were intranasally infected with a cowpox virus that is lethal for this non-human primate species and designated calpox virus. The calpox virus/marmoset model was established to study pathogenesis and to evaluate new vaccines and therapeutics.

The aim of this doctoral thesis is to contribute to the understanding of the pathogenesis of the calpox virus infection in CM and other OPXV infections in humans. The calpox virus/marmoset model was used to (i) determine the portal of viral entry, (ii) analyze the early dissemination as well as the pathological sequelae of infection in a time-course pathogenesis study. The purpose of this research is also to (iii) analyze the immune response of the different vaccines and vaccination routes and to identify immune correlates of protection in two vaccination studies. The calpox virus/marmoset model, a hypothesized model for smallpox or other OPXV infections in humans was used to evaluate the protective effect of different attenuated vaccines.

For the pathogenesis study two groups of three animals each were formed. First group was infected with a $3.5 \times 10^{5}$ pfu calpox virus (low dose group) and euthanized on day 3 post inoculation. Second group was challenged with $8.3 \times 10^{3}$ pfu calpox virus (high dose group) and euthanized on days $5,7,10$ or 12 post infection. Blood and various organs were collected from the animals and analyzed for infectious virus using a plaque assay, endpoint dilution assay and for viral DNA using real-time PCR. PBMCs and buffy coat were analyzed for the calpox virus antigen in immune cells by flow cytometry.

The earliest replicating virus was detectable at the inoculation site, the nasal mucosa and at a much lower levels in the esophagus and tongue at 3 dpi after the high dose challenge. After the low dose challenge, earliest viral DNA was detected in the nasal mucosa, submandibular lymph nodes and tonsils at $5 \mathrm{dpi}$ and in blood and several organs at 7, 10 and $12 \mathrm{dpi}$. In animals received high dose infection, double positive $\mathrm{CD}^{+} \mathrm{CD} 20^{+}$cells and intermediate 
$\mathrm{CD} 14^{+} \mathrm{CD} 16^{+}$monocytes were already VACV antigen positive $\left(\right.$calpox $\left.^{+}\right)$. In late state of infection (12 dpi) multiple immune cell subsets were tested positive for vaccinia virus antigen. In the performed vaccination studies, two attenuated smallpox vaccines, i.e. modified vaccinia virus (VV) Tian Tan (MVTT) and modified VV Ankara (MVA) were tested for their efficacy after a 4- and a 10-week waiting period. Binding antibodies were quantified with enzymelinked immunosorbent assay (ELISA). For the analysis of neutralizing antibodies (nAb's) was $\mathrm{B}$ cell epitope fine mapping performed with peptide microarray as well as plaque reduction neutralization test (PRNT). The cellular immune response was analyzed by flow cytometric analysis of innate and adaptive immune cells in blood as well as T cell proliferation assay.

When testing the different smallpox vaccines an overall protection of $67 \%$ was observed following immunization with MVTT and $13 \%$ after MVA vaccination, independently of the vaccination route and waiting period. All vaccine failures became virus positive by real-time PCR. MVTT-immunized vaccine failures had lower viral titres in blood than MVA immunized vaccine failures at the final stage. Binding antibodies against viral particles measured by ELISA were not associated with the protection of the CM from the calpox virus challenge. In both vaccination studies binding antibodies did not significantly differ between the vaccine groups at day of challenge. Peptide microarrays revealed antibodies against linear B cell epitope regions in different proteins (A33, B5 and L1) that were exclusively found in protected animals post challenge. Neutralizing antibodies were detected in both vaccination studies at day of challenge by PRNT but no correlation of measured titres between protected and unprotected animals was detected. When serum samples were tested against VACV Lister Elstree (LE), the titres of the MVTT per oral (p.o.) immunized group were significantly higher ( $p=0.0487)$ than the titres of the MVA intramuscularly (i.m.) immunized group at challenge. The cellular response was analyzed by flow cytometric staining. T and B cell numbers did not differ significantly between vaccination groups. The expression of activation markers (e.g. NK cells, $\gamma \delta$ T cells, CD3 ${ }^{+}$cells, CD69, HLA-DR and CD80) were detected at the day of challenge. Virus-specific T-cell proliferation was observed in some animals vaccinated with MVTT, but the proliferative activity was not associated with the protection of the CM from the calpox virus challenge.

In conclusion, the data suggests that the calpox virus initially replicated in the upper respiratory tract followed by systemic spread. The calpox virus probably first replicated in the nasal mucosa and spread from the nasal mucosa to the surrounding lymphatic tissue (submandibular lymph node and tonsil), where secondary replication occured. Subsequently, the virus probably spreads to other organs of the respiratory tract and then to surrounding organs (eyes, 
esophagus, lung and tongue). In the prefinal phase, all organs became infected. Furthermore, the onset of viremia (DNA copies in blood), is probably dose dependent and starts at 3 dpi (with the high inoculation dose) and at $7 \mathrm{dpi}$ (with the low inoculation dose) in this model. The overall highest protection mediated by live-attenuated VV was observed with MVTT independently of the vaccination route and waiting period. Little is known about the immune response in $\mathrm{CM}$ after poxvirus infection and unfortunately, no obvious immune correlates of protection were found in this study.

Since peptide microarrays revealed antibodies against linear B cell epitope regions in different proteins that were present exclusively in protected animals post challenge, neutralizing antibodies seem to play an important role in poxvirus protection. Details of the immunology of calpox virus pathogenesis (e.g. the role of cytokines in the calpox virus/marmoset model) have not been studied so far and due to the limited availability of reagents for CM, their elucidation is difficult and remains a future object for investigation. This study suggests, that recombinant proteins known as protective and neutralizing antibody-inducing determinants against linear epitopes should be analyzed. Further studies could also verify if the efficacy of MVTT immunization can be increased through systemic vaccination. Another thirdgeneration vaccine (e.g. the Japanese strain $\mathrm{LC} 16 \mathrm{~m} 8$ ) may represent another option to test for the efficacy in the calpox virus/marmoset model. 


\section{ZUSAMMENFASSUNG}

Li Lin Gan (2017)

Die Pocken wurden 1980 erfolgreich ausgerottet und Massenimpfungen wurden in Europa zwischen 1976 und 1984 eingestellt. Seitdem nahm die Herdenimmunität gegen Orthopockenviren (OPV) ab und hinterlässt die Population gegen Infektionen des Orthopockenvirus ungeschützt. In Anbetracht des Bioterrorismus und neu entstehender zoonotischen Erkrankungen entsteht ein wachsendes Interesse an der Entwicklung von antiviralen Medikamenten und sichereren Impfstoffen gegen Orthopockenviren. In Zusammenarbeit mit dem Robert Koch Institut (RKI) wurde ein nicht-menschliches Primatenmodell etabliert. Die Weißbüschelaffen (WA, Callithrix jacchus) wurden intranasal mit einem Kuhpockenvirus infiziert, welches für diese nicht-humane Primatenart tödlich ist und Calpox Virus genannt wurde. Das Calpox Virus/Marmosetten Modell wurde etabliert um die Pathogenese und die Evaluierung von neuen Impfstoffen und Therapeutika zu studieren.

Das Ziel dieser Doktorarbeit ist, zu dem Verständnis der Pathogenese der Calpox Virus Infektion in WA und anderen OPV-Infektionen in Menschen beizutragen. Das Calpox Virus/Marmosetten Modell wird benutzt um (i) die virale Eintrittspforte zu ermitteln, (ii) die frühe Verbreitung sowie die pathologischen Folgen der Infektion in einem Zeitverlauf einer Pathogenesestudie zu analysieren. Auch die (iii) Analyse der Immunantwort der verschiedenen Impfstoffe und Impfrouten sowie die Identifizierung von schützenden Immunkorrelaten in zwei Impfstudien ist der Zweck dieser Forschung. Das Calpox Virus/Marmosetten Modell, ein hypothetisches Modell für Pocken oder andere OPV-Infektionen beim Menschen, wurde verwendet um die schützende Wirkung von unterschiedlich abgeschwächten Impfstoffen zu bewerten.

Für die Pathogenesestudie wurden zwei Gruppen mit jeweils drei Tieren gebildet. Die erste Gruppe wurde mit $3,5 \times 10^{5}$ pfu des Calpox Virus infiziert (niedrige Dosis) und am Tag 3 nach der Infektion euthanasiert. Die zweite Gruppe wurde mit $8,3 \times 10^{3}$ pfu des Calpox Virus infiziert (hohe Dosis) und an den Tagen 5, 7, 10 und 12 nach der Infektion euthanasiert. Von den Tieren wurden Blut und verschiedene Organe asserviert und mit dem Plaque-Assay, der Endpunkt-Verdünnungsanalyse, auf infektiöses Virus und mit Real-Time PCR auf virale DNA untersucht. PBMCs und Buffy Coat wurden mit der Durchflusszytometrie auf Calpox Virus Antigen in Immunzellen analysiert.

Mit der niedrigen Infektionsdosis wurde am Tag 5 nach der Infektion die früheste virale DNA in der Nasenschleimhaut, submandibulären Lymphknoten und Tonsille nachweisbar und in 
Blut und vielen Organen an den Tagen 7, 10 und 12 nach der Infektion. Die höchsten viralen DNA-Kopien wurden in Tieren nachgewiesen, die am Tag 12 nach der Infektion euthanasiert wurden.

In Tieren, die mit der hohen Inkeftionsdosis behandelt wurden, waren die doppelten positiven $\mathrm{CD}^{+} \mathrm{CD} 20^{+}$-Zellen und intermediären-CD14 ${ }^{+} \mathrm{CD} 16^{+}$-Monozyten an Tag 3 nach der Infektion bereits Calpox Virus-Antigen-positiv gefärbt. Im späten Zustand der Infektion (12 Tage nach Infektion) waren mehrere Unterguppen der Immunzellen Calpox Virus-Antigen-positiv.

In den durchgeführten Impfstudien wurden zwei attenuierte Pockenimpfstoffe, d. h. modifiziertes Vaccinia-Virus (VV) Tian Tan (MVTT) und modifiziertes VV Ankara (MVA) nach einer 4- und 10-wöchigen Wartezeit auf ihre Wirksamkeit getestet. Bindende Antikörper wurden durch den enzyme-linked immunosorbent assay (ELISA) quantifiziert. Für die Untersuchung von neutralisierenden Antikörpern (nAb's) wurde die B-Zell-Epitop-Feinkartierung mit dem Peptide-Mikroarray durchgeführt sowie der Plaque-Reduktions-Neutralisationstest (PRNT). Die zelluläre Antwort der angeborenen und adaptiven Immunantwort im Blut wurde mit der Durchflusszytometrieanalyse und dem T-Zell-Proliferationstest analysiert.

Beim Testen der verschiedenen Pockenimpfstoffe wurde, unabhängig von der Impfroute und der Wartezeit, nach der Immunisierung mit MVTT $67 \%$-iger Schutz und $13 \%$-iger Schutz nach der MVA-Impfung beobachtet. Alle Impfdurchbrüche wurden in der Real-Time PCR Virus positive. Infektiöses Virus wurde im Blut nachgewiesen und in der Endphase erreichten MVTT-immunisierte Impfdurchbrüche im Blut niedrigere Virus-Titre durch den PlaqueAssay als MVA-immunisierte Impfdurchbrüche. Bindende Antikörper gegen virale Partikel, die durch ELISA gemessen wurden, waren nicht mit dem Schutz des WA gegen den Calpox Virus Challenge assoziiert. In beiden Impfstudien waren die bindenden Antikörper zwischen den Impfgruppen zum Zeitpunkt des Challenge nicht signifikant unterschiedlich. PeptidMikroarrays zeigten Antikörper gegen lineare B-Zell-Epitop-Regionen in verschiedenen Proteinen (A33, B5 und L1), die ausschließlich bei geschützten Tieren nach dem Challenge vorhanden waren. Neutralisierende Antikörper wurden an dem Tag des Challenge in beiden Impfstudien mit dem PRNT nachgewiesen, jedoch wurde keine Korrelation der gemessenen Titre zwischen geschützten und ungeschützten Tieren nachgewiesen. Zum Zeitpunkt des Challenge war der MVTT per oral (p.o.) immunisierte Gruppen Titre signifikant höher (p = 0,0487) als der MVA intramuskulär (i.m.) immunisierte Gruppen Titre, als die Serumproben gegen VACV Lister Elstree (LE) getestet wurden.

Die zelluläre Antwort wurde mit der durchflusszytometrischen Färbung untersucht. T und B Zellzahlen zeigten keine signifikanten Unterschiede zwischen den Impfgruppen. Am Tag des 
Challenge wurde die Expression der getesteten Aktivierungsmarker (z. B. NK-Zellen, $\gamma \delta-T-$ Zellen, CD3 ${ }^{+}$-Zellen, CD69, HLA-DR und CD80) nachgewiesen. Virusspezifische T-ZellProliferation wurde bei einigen Tieren beobachtet, die mit MVTT geimpft wurden, aber die proliferative Aktivität war nicht mit dem Schutz des WA vor dem Calpox Virus Challenge assoziiert.

Folglich deuten die Daten darauf hin, dass das Calpox Virus zunächst in den oberen Atemwegen repliziert, gefolgt von systemischer Ausbreitung. Calpox Virus repliziert vermutlich zuerst in der Nasenschleimhaut und breitet sich von der Nasenschleimhaut zum umgebenden lymphatischen Gewebe (submandibuläre Lymphknoten und Tonsille) aus, wo es zur sekundären Replikation führt. Das Virus verbreitet sich wahrscheinlich durch eine Viremie von dort $\mathrm{zu}$ anderen Organen der Atemwege und anderen umgebenden Organen (Augen, Speiseröhre, Lunge und Zunge). In der präfinalen Phase wurden alle Organe infiziert. Außerdem ist der Beginn der Virämie (DNA Kopien im Blut) wahrscheinlich dosisabhängig und startet 3 Tage nach der Infektion (mit der hohen Dosis) und 7 Tage nach der Infektion (mit der niedrigen Dosis). Insgesamt wurde durch lebendiges abgeschwächtes VV der höchste Schutz durch MVTT beobachtet, unabhängig von der Impfroute und Wartezeit. In Weißbüschelaffen ist über die Immunantwort nach der Pockenvirus-Infektion wenig bekannt und leider wurden in dieser Studie keine offensichtlich schützenden Immunkorrelate gefunden.

Neutralisierende Antikörper scheinen mit dem Schutz gegen Pockenviren eine wichtige Rolle zu spielen, da der Peptid-Mikroarray Antikörper gegen lineare B-Zell-Epitop-Regionen in verschiedenen Proteinen zeigte, die ausschließlich in geschützten Tieren nach dem Challenge vorhanden waren. Einzelheiten der Immunologie der Calpox Virus-Pathogenese (z. B. die Rolle von Cytokinen im Calpox Virus/Marmosetten Modell) wurden bisher auch noch nicht untersucht und sind aufgrund der begrenzten Verfügbarkeit von Reagenzien für WA schwer zu ermitteln und bleiben deswegen Gegenstand künftiger Untersuchungen. Weitere Studien könnten auch zeigen, ob die Wirksamkeit der MVTT-Immunisierung durch eine systemische Impfung erhöht werden kann. Ein Impfstoff der dritten Generation (z. B. der japanische Stamm LC16m8) könnte eine weitere zu testende Möglichkeit repräsentieren, der auch auf die Wirksamkeit im Calpox Virus/Marmosetten Modell analysiert werden könnte. 


\section{LIST OF REFERENCES}

\subsection{Literature cited}

Abbott DH, Barnett DK, Colman RJ, Yamamoto ME, Schultz-Darken NJ. 2003. Aspects of common marmoset basic biology and life history important for biomedical research. Comparative medicine 53(4):339-350.

Agrawal P, Nawadkar R, Ojha H, Kumar J, Sahu A. 2017. Complement Evasion Strategies of Viruses: An Overview. Frontiers in microbiology 8:1117.

Alcami A, Koszinowski UH. 2000. Viral mechanisms of immune evasion. Trends in microbiology 8(9):410-418.

Alcami A, Smith GL. 1992. A soluble receptor for interleukin-1 beta encoded by vaccinia virus: a novel mechanism of virus modulation of the host response to infection. Cell 71(1):153-167.

Aldaz-Carroll L, Whitbeck JC, Ponce de Leon M, Lou H, Hirao L, Isaacs SN, Moss B, Eisenberg RJ, Cohen GH. 2005. Epitope-mapping studies define two major neutralization sites on the vaccinia virus extracellular enveloped virus glycoprotein B5R. Journal of virology 79(10):6260-6271.

Aldaz-Carroll L, Xiao Y, Whitbeck JC, de Leon MP, Lou H, Kim M, Yu J, Reinherz EL, Isaacs SN, Eisenberg RJ and others. 2007. Major neutralizing sites on vaccinia virus glycoprotein B5 are exposed differently on variola virus ortholog B6. Journal of virology 81(15):8131-8139.

Amanna IJ, Slifka MK, Crotty S. 2006. Immunity and immunological memory following smallpox vaccination. Immunological reviews 211:320-337.

Antoine G, Scheiflinger F, Dorner F, Falkner FG. 1998. The complete genomic sequence of the modified vaccinia Ankara strain: comparison with other orthopoxviruses. Virology 244(2):365-396.

Balamurugan V, Venkatesan G, Bhanuprakash V, Singh RK. 2013. Camelpox, an emerging orthopox viral disease. Indian journal of virology : an official organ of Indian Virological Society 24(3):295-305.

Baldick CJ, Jr., Moss B. 1993. Characterization and temporal regulation of mRNAs encoded by vaccinia virus intermediate-stage genes. Journal of virology 67(6):3515-3527.

Barnewall RE, Fisher DA, Robertson AB, Vales PA, Knostman KA, Bigger JE. 2012. Inhalational monkeypox virus infection in cynomolgus macaques. Frontiers in cellular and infection microbiology 2:117.

Barquet N, Domingo P. 1997. Smallpox: the triumph over the most terrible of the ministers of death. Annals of internal medicine 127(8 Pt 1):635-642.

Baxby D. 1977. Poxvirus hosts and reservoirs. Brief review. Archives of virology 55(3):169179.

Baxby D, Ashton DG, Jones D, Thomsett LR, Denham EM. 1979. Cowpox virus infection in unusual hosts. The Veterinary record 104(8):175.

Baxby D, Bennett M. 1990. Low risk from feline cowpox. Lancet (London, England) 336(8722):1070-1071.

Baxby D, Bennett M, Getty B. 1994. Human cowpox 1969-93: a review based on 54 cases. The British journal of dermatology 131(5):598-607.

Becker C, Kurth A, Hessler F, Kramp H, Gokel M, Hoffmann R, Kuczka A, Nitsche A. 2009. Cowpox virus infection in pet rat owners: not always immediately recognized. Deutsches Arzteblatt international 106(19):329-334. 
Begon M, Hazel SM, Baxby D, Bown K, Cavanagh R, Chantrey J, Jones T, Bennett M. 1999. Transmission dynamics of a zoonotic pathogen within and between wildlife host species. Proceedings Biological sciences 266(1432):1939-1945.

Belongia EA, Naleway AL. 2003. Smallpox vaccine: the good, the bad, and the ugly. Clinical medicine \& research 1(2):87-92.

Benhnia MR, McCausland MM, Moyron J, Laudenslager J, Granger S, Rickert S, Koriazova L, Kubo R, Kato S, Crotty S. 2009. Vaccinia virus extracellular enveloped virion neutralization in vitro and protection in vivo depend on complement. Journal of virology 83(3):1201-1215.

Bera BC, Shanmugasundaram K, Barua S, Venkatesan G, Virmani N, Riyesh T, Gulati BR, Bhanuprakash V, Vaid RK, Kakker NK and others. 2011. Zoonotic cases of camelpox infection in India. Veterinary microbiology 152(1-2):29-38.

Bhanuprakash V, Venkatesan G, Balamurugan V, Hosamani M, Yogisharadhya R, Chauhan RS, Pande A, Mondal B, Singh RK. 2010. Pox outbreaks in sheep and goats at Makhdoom (Uttar Pradesh), India: evidence of sheeppox virus infection in goats. Transboundary and emerging diseases 57(5):375-382.

Bienenstock J, McDermott MR. 2005. Bronchus- and nasal-associated lymphoid tissues. Immunological reviews 206:22-31.

Blackford S, Roberts DL, Thomas PD. 1993. Cowpox infection causing a generalized eruption in a patient with atopic dermatitis. The British journal of dermatology 129(5):628-629.

Bonnekoh B, Falk K, Reckling KF, Kenklies S, Nitsche A, Ghebremedhin B, Pokrywka A, Franke I, Thriene B, Konig W and others. 2008. Cowpox infection transmitted from a domestic cat. Journal der Deutschen Dermatologischen Gesellschaft $=$ Journal of the German Society of Dermatology : JDDG 6(3):210-213.

Bourquain D, Dabrowski PW, Nitsche A. 2013. Comparison of host cell gene expression in cowpox, monkeypox or vaccinia virus-infected cells reveals virus-specific regulation of immune response genes. Virology journal 10:61.

Bray M, Buller M. 2004. Looking back at smallpox. Clinical infectious diseases : an official publication of the Infectious Diseases Society of America 38(6):882-889.

Bray M, Wright ME. 2003. Progressive vaccinia. Clinical infectious diseases : an official publication of the Infectious Diseases Society of America 36(6):766-774.

Breman JG. 2000. Monkeypox: an emerging infection for humans?, Scheld W.M., Craig W.A., Hughes J.M. Emerging infections ASM Press, Washington DC 4:45-67, .

Broyles SS. 2003. Vaccinia virus transcription. The Journal of general virology 84(Pt 9):2293-2303.

Buchman GW, Cohen ME, Xiao Y, Richardson-Harman N, Silvera P, DeTolla LJ, Davis HL, Eisenberg RJ, Cohen GH, Isaacs SN. 2010. A protein-based smallpox vaccine protects non-human primates from a lethal monkeypox virus challenge. Vaccine 28(40):66276636.

Buller RM, Palumbo GJ. 1991. Poxvirus pathogenesis. Microbiological reviews 55(1):80-122. Callaway E. 2008. Bioterror: the green menace. Nature 452(7184):148-150.

Campe H, Zimmermann P, Glos K, Bayer M, Bergemann H, Dreweck C, Graf P, Weber BK, Meyer H, Buttner M and others. 2009. Cowpox virus transmission from pet rats to humans, Germany. Emerging infectious diseases 15(5):777-780.

Carrion R, Jr., Brasky K, Mansfield K, Johnson C, Gonzales M, Ticer A, Lukashevich I, Tardif S, Patterson J. 2007. Lassa virus infection in experimentally infected CM: liver pathology and immunophenotypic alterations in target tissues. Journal of virology 81(12):6482-6490. 
Carroll DS, Emerson GL, Li Y, Sammons S, Olson V, Frace M, Nakazawa Y, Czerny CP, Tryland M, Kolodziejek J and others. 2011. Chasing Jenner's vaccine: revisiting cowpox virus classification. PLoS One 6(8):e23086.

Casemore DP, Emslie ES, Whyler DK, Baxby D, Bennett M, Davies AB. 1987. Cowpox in a child, acquired from a cat. Clinical and experimental dermatology 12(4):286-287.

CDC. 2008. Bioterrorism Argents/Diseases. (10/08/2008).

Chan WM, Ward BM. 2010. There is an A33-dependent mechanism for the incorporation of B5-GFP into vaccinia virus extracellular enveloped virions. Virology 402(1):83-93.

Chantrey J, Meyer H, Baxby D, Begon M, Bown KJ, Hazel SM, Jones T, Montgomery WI, Bennett M. 1999. Cowpox: reservoir hosts and geographic range. Epidemiology and infection 122(3):455-460.

Chung CS, Hsiao JC, Chang YS, Chang W. 1998a. A27L protein mediates vaccinia virus interaction with cell surface heparan sulfate. Journal of virology 72 (2):1577-1585.

Chung CS, Hsiao JC, Chang YS, Chang W. 1998b. A27L protein mediates vaccinia virus interaction with cell surface heparan sulfate. Journal of virology 72(2):1577-1585.

Collier LH. 1955. The Development of a Stable Smallpox Vaccine. The Journal of hygiene(53.1):76-101.

Condit RC, Moussatche N, Traktman P. 2006. In a nutshell: structure and assembly of the vaccinia virion. Advances in virus research 66:31-124.

Cosma A, Nagaraj R, Buhler S, Hinkula J, Busch DH, Sutter G, Goebel FD, Erfle V. 2003. Therapeutic vaccination with MVA-HIV-1 nef elicits Nef-specific T-helper cell responses in chronically HIV-1 infected individuals. Vaccine 22(1):21-29.

Councilman WT, Magrath GB, Brinckerhoff WR. 1904. The Pathological Anatomy and Histology of Variola. The Journal of medical research 11(1):12-135.

Craighead JE. 2000. Pathology and pathogenesis of human viral disease.366.

Crotty S, Felgner P, Davies H, Glidewell J, Villarreal L, Ahmed R. 2003. Cutting edge: longterm B cell memory in humans after smallpox vaccination. Journal of immunology 171(10):4969-4973.

Czerny CP, Eis-Hubinger AM, Mayr A, Schneweis KE, Pfeiff B. 1991. Animal poxviruses transmitted from cat to man: current event with lethal end. Zentralblatt fur Veterinarmedizin Reihe B Journal of veterinary medicine Series B 38(6):421-431.

Davies DH, McCausland MM, Valdez C, Huynh D, Hernandez JE, Mu Y, Hirst S, Villarreal L, Felgner PL, Crotty S. 2005. Vaccinia virus H3L envelope protein is a major target of neutralizing antibodies in humans and elicits protection against lethal challenge in mice. Journal of virology 79(18):11724-11733.

Davies DH, Molina DM, Wrammert J, Miller J, Hirst S, Mu Y, Pablo J, Unal B, NakajimaSasaki R, Liang X and others. 2007. Proteome-wide analysis of the serological response to vaccinia and smallpox. Proteomics 7(10):1678-1686.

Davies ML, Parekh NJ. 2017. A systemic macrophage response is required to contain a peripheral poxvirus infection. 13(6):e1006435.

Demkowicz WE, Maa JS, Esteban M. 1992. Identification and characterization of vaccinia virus genes encoding proteins that are highly antigenic in animals and are immunodominant in vaccinated humans. Journal of virology 66(1):386-398.

Deria A, Jezek Z, Markvart K, Carrasco P, Weisfeld J. 1980. The world's last endemic case of smallpox: surveillance and containment measures. Bulletin of the World Health Organization 58(2):279-283.

Digby LJ, Barreto CE. 1993. Social organization in a wild population of Callithrix jacchus. I. Group composition and dynamics. Folia primatologica; international journal of primatology 61(3):123-134. 
Doms RW, Blumenthal R, Moss B. 1990. Fusion of intra- and extracellular forms of vaccinia virus with the cell membrane. Journal of virology 64(10):4884-4892.

Downie AW. 1939. The Immunological Relationship of the Virus of Spontaneous Cowpox to Vaccinia Virus. British Journal of Experimental Pathology(20.2):158-176.

Downie AW, Mc CK. 1958. The antibody response in man following infection with viruses of the pox group. III. Antibody response in smallpox. The Journal of hygiene 56(4):479487.

Downie AW, McCarthy K, Macdonald A, Maccallum FO, Macrae AE. 1953. Virus and virus antigen in the blood of smallpox patients; their significance in early diagnosis and prognosis. Lancet (London, England) 265(6778):164-166.

Downie AW, Saint Vincent L, Goldstein L, Rao AR, Kwmpw CH. 1969. Antibody response in non-haemorrhagic smallpox patients. J Hyg 67:609-618.

Dugmore WN, Dabir ZM. 1992. Cowpox virus. The British journal of ophthalmology 76(8):510.

Earl PL, Americo JL, Wyatt LS, Eller LA, Whitbeck JC, Cohen GH, Eisenberg RJ, Hartmann CJ, Jackson DL, Kulesh DA and others. 2004. Immunogenicity of a highly attenuated MVA smallpox vaccine and protection against monkeypox. Nature 428(6979):182185.

Earl PL, Americo JL, Wyatt LS, Espenshade O, Bassler J, Gong K, Lin S, Peters E, Rhodes L, Jr., Spano YE and others. 2008. Rapid protection in a monkeypox model by a single injection of a replication-deficient vaccinia virus. Proceedings of the National Academy of Sciences of the United States of America 105(31):10889-10894.

Edghill-Smith Y, Golding H, Manischewitz J, King LR, Scott D, Bray M, Nalca A, Hooper JW, Whitehouse CA, Schmitz JE and others. 2005. Smallpox vaccine-induced antibodies are necessary and sufficient for protection against monkeypox virus. Nature medicine 11(7):740-747.

el-Ad B, Roth Y, Winder A, Tochner Z, Lublin-Tennenbaum T, Katz E, Schwartz T. 1990. The persistence of neutralizing antibodies after revaccination against smallpox. The Journal of infectious diseases 161(3):446-448.

Engelstad M, Howard ST, Smith GL. 1992. A constitutively expressed vaccinia gene encodes a 42-kDa glycoprotein related to complement control factors that forms part of the extracellular virus envelope. Virology 188(2):801-810.

Ennis FA, Cruz J, Demkowicz WE, Jr., Rothman AL, McClain DJ. 2002. Primary induction of human CD8+ cytotoxic T lymphocytes and interferon-gamma-producing T cells after smallpox vaccination. The Journal of infectious diseases 185(11):1657-1659.

Eslamboli A. 2005. Marmoset monkey models of Parkinson's disease: which model, when and why? Brain research bulletin 68(3):140-149.

Essbauer S, Pfeffer M, Meyer H. 2010. Zoonotic poxviruses. Veterinary microbiology 140(34):229-236.

Essbauer S, Pfeffer M, Wilhelm S, Meyer H. 2004. [Zoonotic poxviruses]. Bundesgesundheitsblatt, Gesundheitsforschung, Gesundheitsschutz 47(7):671-679.

Fang M, Sigal LJ. 2006. Direct CD28 costimulation is required for CD8+ T cell-mediated resistance to an acute viral disease in a natural host. Journal of immunology 177(11):8027-8036.

Fang Q, Yang L, Zhu W, Liu L, Wang H, Yu W, Xiao G, Tien P, Zhang L, Chen Z. 2005. Host range, growth property, and virulence of the smallpox vaccine: vaccinia virus Tian Tan strain. Virology 335(2):242-251.

Fenner F, Henderson DA, Arita I, Jezek Z, Ladnyi ID. 1988a. Human monkeypox and other poxvirus infections of man, Smallpox and its Eradication. World Health Organization, Geniva, Switzerland:1287-1320. 
Fenner F, Henderson DA, Arita I, Jezek Z, Ladnyi ID. 1988b. Pathogenesis, pathology and immunology of smallpox. Smallpox and its Eradication. Geneva: WHO. p 121-168.

Fenner F, Henderson DA, Arita I, Jezek Z, Ladnyi ID. 1988b. Smallpox vaccine and vaccination in the intensified smallpox

eradication programme. In Smallpox and its Eradication. Geneva: World Health Organization:pp. 539-592.

Fenner F, Henderson DA, Arita I, Jezek Z, Ladnyi ID, Organization WH. 1988c. Smallpox and its eradication. Geneva: World Health Organization.

Fine PE, Jezek Z, Grab B, Dixon H. 1988. The transmission potential of monkeypox virus in human populations. International journal of epidemiology 17(3):643-650.

Fogg C, Lustig S, Whitbeck JC, Eisenberg RJ, Cohen GH, Moss B. 2004. Protective immunity to vaccinia virus induced by vaccination with multiple recombinant outer membrane proteins of intracellular and extracellular virions. Journal of virology 78(19):10230-10237.

Fogg CN, Americo JL, Lustig S, Huggins JW, Smith SK, Damon I, Resch W, Earl PL, Klinman DM, Moss B. 2007. Adjuvant-enhanced antibody responses to recombinant proteins correlates with protection of mice and monkeys to orthopoxvirus challenges. Vaccine 25(15):2787-2799.

Frey SE, Newman FK, Kennedy JS, Sobek V, Ennis FA, Hill H, Yan LK, Chaplin P, Vollmar J, Chaitman BR and others. 2007. Clinical and immunologic responses to multiple doses of IMVAMUNE (Modified Vaccinia Ankara) followed by Dryvax challenge. Vaccine 25(51):8562-8573.

Frey SE, Newman FK, Yan L, Lottenbach KR, Belshe RB. 2003. Response to smallpox vaccine in persons immunized in the distant past. Jama 289(24):3295-3299.

Frey SE, Winokur PL, Salata RA, El-Kamary SS, Turley CB, Walter EB, Jr., Hay CM, Newman FK, Hill HR, Zhang Y and others. 2013. Safety and immunogenicity of IMVAMUNE(R) smallpox vaccine using different strategies for a post event scenario. Vaccine 31(29):3025-3033.

Fridholm H, Everitt E. 2005. Rapid and reproducible infectivity end-point titration of virulent phage in a microplate system J Virol Methods 128(1-2): 67-71.

Galasso GJ, Tyeryar FJ, Jr., La Montagne JR. 1977. Overview of clinical trials of influenza vaccines, 1976. The Journal of infectious diseases 136 Suppl:S425-428.

Galmiche MC, Goenaga J, Wittek R, Rindisbacher L. 1999. Neutralizing and protective antibodies directed against vaccinia virus envelope antigens. Virology 254(1):71-80.

Garon CF, Barbosa E, Moss B. 1978. Visualization of an inverted terminal repetition in vaccinia virus DNA. Proceedings of the National Academy of Sciences of the United States of America 75(10):4863-4867.

Garza NL, Hatkin JM, Livingston V, Nichols DK, Chaplin PJ, Volkmann A, Fisher D, Nalca A. 2009. Evaluation of the efficacy of modified vaccinia Ankara (MVA)/IMVAMUNE against aerosolized rabbitpox virus in a rabbit model. Vaccine 27(40):5496-5504.

Gaylord WH, Jr., Melnick JL. 1953. Developmental forms of vaccinia virus. Science (New York, NY) 117(3027):10-13.

Genain CP, Hauser SL. 1997. Creation of a model for multiple sclerosis in Callithrix jacchus CM. Journal of molecular medicine (Berlin, Germany) 75(3):187-197.

Gilchuk I, Gilchuk P, Sapparapu G, Lampley R, Singh V, Kose N, Blum DL, Hughes LJ, Satheshkumar PS, Townsend MB and others. 2016a. Cross-Neutralizing and Protective Human Antibody Specificities to Poxvirus Infections. Cell 167(3):684694.e689. 
Gilchuk I, Gilchuk P, Sapparapu G, Lampley R, Singh V, Kose N, Blum DL, Hughes LJ, Satheshkumar PS, Townsend MB and others. 2016b. Cross-Neutralizing and Protective Human Antibody Specificities to Poxvirus Infections. Cell 167(3):684-694 e689.

Golovkin M, Spitsin S, Andrianov V, Smirnov Y, Xiao Y, Pogrebnyak N, Markley K, Brodzik R, Gleba Y, Isaacs SN and others. 2007. Smallpox subunit vaccine produced in Planta confers protection in mice. Proceedings of the National Academy of Sciences of the United States of America 104(16):6864-6869.

Gong SC, Lai CF, Esteban M. 1990. Vaccinia virus induces cell fusion at acid pH and this activity is mediated by the $\mathrm{N}$-terminus of the $14-\mathrm{kDa}$ virus envelope protein. Virology 178(1):81-91.

Gordon SN, Cecchinato V, Andresen V, Heraud JM, Hryniewicz A, Parks RW, Venzon D, Chung HK, Karpova T, McNally J and others. 2011. Smallpox vaccine safety is dependent on T cells and not B cells. The Journal of infectious diseases 203(8):10431053.

Goyal T, Varshney A, Bakshi SK, Barua S, Bera BC, Singh RK. 2013. Buffalo pox outbreak with atypical features: a word of caution and need for early intervention. Int J Dermatol 52(10):1224-1230.

Greenberg RN, Overton ET, Haas DW, Frank I, Goldman M, von Krempelhuber A, Virgin G, Badeker N, Vollmar J, Chaplin P. 2013. Safety, immunogenicity, and surrogate markers of clinical efficacy for modified vaccinia Ankara as a smallpox vaccine in HIV-infected subjects. The Journal of infectious diseases 207(5):749-758.

Gubser C, Hue S, Kellam P, Smith GL. 2004. Poxvirus genomes: a phylogenetic analysis. The Journal of general virology 85(Pt 1):105-117.

Haga IR, Bowie AG. 2005. Evasion of innate immunity by vaccinia virus. Parasitology 130 Suppl:S11-25.

Hahon N, Wilson BJ. 1960. Pathogenesis of variola in Macaca irus monkeys. American journal of hygiene 71:69-80.

Hall CJ, Stevens JD. 1987. Ocular cowpox. Lancet (London, England) 1(8524):111.

Hammarlund E, Lewis MW, Carter SV, Amanna I, Hansen SG, Strelow LI, Wong SW, Yoshihara P, Hanifin JM, Slifka MK. 2005. Multiple diagnostic techniques identify previously vaccinated individuals with protective immunity against monkeypox. Nature medicine 11(9):1005-1011.

Hammarlund E, Lewis MW, Hanifin JM, Mori M, Koudelka CW, Slifka MK. 2010. Antiviral immunity following smallpox virus infection: a case-control study. Journal of virology 84(24):12754-12760.

Hammarlund E, Lewis MW, Hansen SG, Strelow LI, Nelson JA, Sexton GJ, Hanifin JM, Slifka MK. 2003. Duration of antiviral immunity after smallpox vaccination. Nature medicine 9(9):1131-1137.

Hanna W, Baxby D. 2002. Studies in smallpox and vaccination. 1913. Reviews in medical virology 12(4):201-209.

Hatch GJ, Graham VA, Bewley KR, Tree JA, Dennis M, Taylor I, Funnell SG, Bate SR, Steeds K, Tipton T and others. 2013. Assessment of the protective effect of Imvamune and Acam 2000 vaccines against aerosolized monkeypox virus in cynomolgus macaques. Journal of virology 87(14):7805-7815.

Henderson DA. 1999. The looming threat of bioterrorism. Science (New York, NY) 283(5406):1279-1282.

Henderson DA. 2011. The eradication of smallpox--an overview of the past, present, and future. Vaccine 29 Suppl 4:D7-9. 
Henderson DA, Inglesby TV, Bartlett JG, Ascher MS, Eitzen E, Jahrling PB, Hauer J, Layton M, McDade J, Osterholm MT and others. 1999. Smallpox as a biological weapon: medical and public health management. Working Group on Civilian Biodefense. Jama 281(22):2127-2137.

Henderson DA, Moss B. 1999. Public Health. Vaccines 3rd edition, Philadelphia: Saunders. Henry C, Ramadan A, Montcuquet N, Pallandre JR, Mercier-Letondal P, Deschamps M, Tiberghien P, Ferrand C, Robinet E. 2010. CD3+CD20+ cells may be an artifact of flow cytometry: comment on the article by Wilk et al. Arthritis and rheumatism 62(8):2561-2563; author reply 2563-2565.

Heraud JM, Edghill-Smith Y, Ayala V, Kalisz I, Parrino J, Kalyanaraman VS, Manischewitz J, King LR, Hryniewicz A, Trindade CJ and others. 2006. Subunit recombinant vaccine protects against monkeypox. Journal of immunology 177(4):2552-2564.

Hiller G, Weber K. 1985. Golgi-derived membranes that contain an acylated viral polypeptide are used for vaccinia virus envelopment. Journal of virology 55(3):651-659.

Hirao LA, Draghia-Akli R, Prigge JT. 2011. Multivalent Smallpox DNA Vaccine Delivered by Intradermal Electroporation Drives Protective Immunity in Nonhuman Primates Against Lethal Monkeypox Challenge. J Infect Dis 203 ((1)):95-102.

Hochstein-Mintzel V, Hanichen T, Huber HC, Stickl H. 1975. [An attenuated strain of vaccinia virus (MVA). Successful intramuscular immunization against vaccinia and variola (author's transl)]. Zentralblatt fur Bakteriologie, Parasitenkunde, Infektionskrankheiten und Hygiene Erste Abteilung Originale Reihe A: Medizinische Mikrobiologie und Parasitologie 230(3):283-297.

Holmgren J, Czerkinsky C. 2005. Mucosal immunity and vaccines. Nature medicine 11(4 Suppl):S45-53.

Hooper JW, Custer DM, Schmaljohn CS, Schmaljohn AL. 2000. DNA vaccination with vaccinia virus L1R and A33R genes protects mice against a lethal poxvirus challenge. Virology 266(2):329-339.

Hooper JW, Custer DM, Thompson E. 2003. Four-gene-combination DNA vaccine protects mice against a lethal vaccinia virus challenge and elicits appropriate antibody responses in nonhuman primates. Virology 306(1):181-195.

Hooper JW, Thompson E, Wilhelmsen C, Zimmerman M, Ichou MA, Steffen SE, Schmaljohn CS, Schmaljohn AL, Jahrling PB. 2004. Smallpox DNA vaccine protects nonhuman primates against lethal monkeypox. Journal of virology 78(9):4433-4443.

Hopkins DR. 1983. Princes and Peasants: Smallpox in History. Chicago: University of Chicago Press.

Hotop SK. 2014. Feinkartierung humoraler Immunantworten von Makaken nach Immunisierung und/oder viraler Infektion mittels Peptid-Microarray. Dissertation.

Hotop SK, Abd El Wahed A, Beutling U, Jentsch D, Motzkus D, Frank R, Hunsmann G, Stahl-Hennig C, Fritz HJ. 2014. Multiple antibody targets on herpes B glycoproteins B and $\mathrm{D}$ identified by screening sera of infected rhesus macaques with peptide microarrays. PLoS One 9(1):e86857.

Hsiao JC, Chung CS, Chang W. 1998. Cell surface proteoglycans are necessary for A27L protein-mediated cell fusion: identification of the $\mathrm{N}$-terminal region of A27L protein as the glycosaminoglycan-binding domain. Journal of virology 72 (10):8374-8379.

Hsiao JC, Chung CS, Chang W. 1999. Vaccinia virus envelope D8L protein binds to cell surface chondroitin sulfate and mediates the adsorption of intracellular mature virions to cells. Journal of virology 73(10):8750-8761.

Huang X, Lu B, Yu W, Fang Q, Liu L, Zhuang K, Shen T, Wang H, Tian P, Zhang L and others. 2009. A novel replication-competent vaccinia vector MVTT is superior to 
MVA for inducing high levels of neutralizing antibody via mucosal vaccination. PLoS One 4(1):e4180.

Hugin AW, Flexner C, Moss B. 1993. Clearance of recombinant vaccinia virus expressing IL2: role of local host immune responses. Cellular immunology 152(2):499-509.

Hutin YJ, Williams RJ, Malfait P, Pebody R, Loparev VN, Ropp SL, Rodriguez M, Knight JC, Tshioko FK, Khan AS and others. 2001. Outbreak of human monkeypox, Democratic Republic of Congo, 1996 to 1997. Emerging infectious diseases 7(3):434438.

Huygelen C. 1996. [Jenner's cowpox vaccine in light of current vaccinology]. Verhandelingen - Koninklijke Academie voor Geneeskunde van Belgie 58(5):479-536; discussion 537478.

Ichihashi Y. 1996. Extracellular enveloped vaccinia virus escapes neutralization. Virology 217(2):478-485.

ICTV. 2017. International Committee on Taxonomy of Viruses (ICTV) EC 47, London, UK, July 2015.

Isaacs SN, Wolffe EJ, Payne LG, Moss B. 1992. Characterization of a vaccinia virus-encoded 42-kilodalton class I membrane glycoprotein component of the extracellular virus envelope. Journal of virology 66(12):7217-7224.

Jagessar SA, Vierboom M, Blezer EL, Bauer J, Hart BA, Kap YS. 2013. Overview of models, methods, and reagents developed for translational autoimmunity research in the common marmoset (Callithrix jacchus). Experimental animals 62(3):159-171.

Jahrling PB, Fritz EA, Hensley LE. 2005. Countermeasures to the bioterrorist threat of smallpox. Current molecular medicine 5(8):817-826.

Jahrling PB, Hensley LE, Martinez MJ, Leduc JW, Rubins KH, Relman DA, Huggins JW. 2004. Exploring the potential of variola virus infection of cynomolgus macaques as a model for human smallpox. Proceedings of the National Academy of Sciences of the United States of America 101(42):15196-15200.

Jezek Z, Arita I, Mutombo M, Dunn C, Nakano JH, Szczeniowski M. 1986. Four generations of probable person-to-person transmission of human monkeypox. American journal of epidemiology 123(6):1004-1012.

Jezek Z, Khodakevich LN, Szczeniowski MV. 1988. [Human monkey pox: its clinicoepidemiological characteristics]. Zhurnal mikrobiologii, epidemiologii, i immunobiologii(6):23-30.

Jezek Z, Kriz B, Rothbauer V. 1983. Camelpox and its risk to the human population. Journal of hygiene, epidemiology, microbiology, and immunology 27(1):29-42.

Jezek Z, Szczeniowski M, Paluku KM, Mutombo M. 1987. Human monkeypox: clinical features of 282 patients. The Journal of infectious diseases 156(2):293-298.

Johnson RF, Hammoud DA, Lackemeyer MG, Yellayi S, Solomon J, Bohannon JK, Janosko KB, Jett C, Cooper K, Blaney JE and others. 2015. Small particle aerosol inoculation of cowpox Brighton Red in rhesus monkeys results in a severe respiratory disease. Virology 481:124-135.

Johnson RF, Yellayi S, Cann JA, Johnson A, Smith AL, Paragas J, Jahrling PB, Blaney JE. 2011. Cowpox virus infection of cynomolgus macaques as a model of hemorrhagic smallpox. Virology 418(2):102-112.

Johnston JB, McFadden G. 2004. Technical knockout: understanding poxvirus pathogenesis by selectively deleting viral immunomodulatory genes. Cellular microbiology 6(8):695-705.

Johnston SC, Johnson JC, Stonier SW, Lin KL, Kisalu NK, Hensley LE, Rimoin AW. 2015. Cytokine modulation correlates with severity of monkeypox disease in humans. 
Journal of clinical virology : the official publication of the Pan American Society for Clinical Virology 63:42-45.

Joklik WK, Becker Y. 1964. The Replication and Coating of Vaccinia DNA. Journal of molecular biology 10:452-474.

Jones-Trower A, Garcia A, Meseda CA, He Y, Weiss C, Kumar A, Weir JP, Merchlinsky M. 2005. Identification and preliminary characterization of vaccinia virus (Dryvax) antigens recognized by vaccinia immune globulin. Virology 343(1):128-140.

Jordan R, Hruby D. 2006. Smallpox antiviral drug development: satisfying the animal efficacy rule. Expert review of anti-infective therapy 4(2):277-289.

Kaever T, Matho MH, Meng X, Crickard L, Schlossman A, Xiang Y, Crotty S, Peters B, Zajonc DM. 2016. Linear Epitopes in Vaccinia Virus A27 Are Targets of Protective Antibodies Induced by Vaccination against Smallpox. Journal of virology 90(9):43344345.

Kalthoff D, Bock WI, Huhn F, Beer M, Hoffmann B. 2014. Fatal cowpox virus infection in cotton-top tamarins (Saguinus oedipus) in Germany. Vector borne and zoonotic diseases (Larchmont, NY) 14(4):303-305.

Kempe CH, Dekking F, Saint Vincent L, Rao AR, Downie AW. 1969. Conjunctivitis and subclinical infection in smallpox. The Journal of hygiene 67(4):631-636.

Kennedy JS, Greenberg RN. 2009. IMVAMUNE: modified vaccinia Ankara strain as an attenuated smallpox vaccine. Expert review of vaccines 8(1):13-24.

Kennedy JS, Gurwith M, Dekker CL, Frey SE, Edwards KM, Kenner J, Lock M, Empig C, Morikawa S, Saijo M and others. 2011. Safety and immunogenicity of LC16m8, an attenuated smallpox vaccine in vaccinia-naive adults. The Journal of infectious diseases 204(9):1395-1402.

Klingebiel T, Vallbracht A, Doller G, Stierhof YD, Gerth HJ, Glashauser E, Herzau V. 1988. A severe human cowpox infection in south Germany. The Pediatric infectious disease journal 7(12):883-885.

Knitlova J, Hajkova V, Voska L, Elsterova J, Obrova B, Melkova Z. 2014. Development of eczema vaccinatum in atopic mouse models and efficacy of MVA vaccination against lethal poxviral infection. PLoS One 9(12):e114374.

Kochan G, Escors D, Gonzalez JM, Casasnovas JM, Esteban M. 2008. Membrane cell fusion activity of the vaccinia virus A17-A27 protein complex. Cellular microbiology 10(1):149-164.

Kortepeter MG, Parker GW. 1999. Potential biological weapons threats. Emerging infectious diseases 5(4):523-527.

Kotwal GJ, Isaacs SN, McKenzie R, Frank MM, Moss B. 1990. Inhibition of the complement cascade by the major secretory protein of vaccinia virus. Science (New York, NY) 250(4982):827-830.

Kramski M. 2009. Infection of Common CM with Calpox Virus: A Model for Smallpox Virus Infection. Dissertation.

Kramski M, Matz-Rensing K, Stahl-Hennig C, Kaup FJ, Nitsche A, Pauli G, Ellerbrok H. 2010. A novel highly reproducible and lethal nonhuman primate model for orthopox virus infection. PLoS One 5(4):e10412.

Kurth A, Straube M, Kuczka A, Dunsche AJ, Meyer H, Nitsche A. 2009. Cowpox virus outbreak in banded mongooses (Mungos mungo) and jaguarundis (Herpailurus yagouaroundi) with a time-delayed infection to humans. PLoS One 4(9):e6883.

Kurth A, Wibbelt G, Gerber HP, Petschaelis A, Pauli G, Nitsche A. 2008. Rat-to-elephant-tohuman transmission of cowpox virus. Emerging infectious diseases 14(4):670-671.

LaBarre DD. 2001. Improvements in methods for calculating virus titer estimates from TCID50 and plaque assays. J Virol Methods 96(2):107-26. 
Langer WL. 1976. Immunization against smallpox before Jenner. Scientific American 234(1):112-117.

Langland JO, Kash JC, Carter V, Thomas MJ, Katze MG, Jacobs BL. 2006. Suppression of proinflammatory signal transduction and gene expression by the dual nucleic acid binding domains of the vaccinia virus E3L proteins. Journal of virology 80(20):1008310095.

Lawrence SJ, Lottenbach KR, Newman FK, Buller RM, Bellone CJ, Chen JJ, Cohen GH, Eisenberg RJ, Belshe RB, Stanley SL, Jr. and others. 2007. Antibody responses to vaccinia membrane proteins after smallpox vaccination. The Journal of infectious diseases 196(2):220-229.

Learned LA, Reynolds MG, Wassa DW, Li Y, Olson VA, Karem K, Stempora LL, Braden $\mathrm{ZH}$, Kline R, Likos A and others. 2005. Extended interhuman transmission of monkeypox in a hospital community in the Republic of the Congo, 2003. The American journal of tropical medicine and hygiene 73(2):428-434.

Leikina E, Ramos C, Markovic I, Zimmerberg J, Chernomordik LV. 2002. Reversible stages of the low-pH-triggered conformational change in influenza virus hemagglutinin. The EMBO journal 21(21):5701-5710.

Lever MS, Stagg AJ, Nelson M, Pearce P, Stevens DJ, Scott EA, Simpson AJ, Fulop MJ. 2008. Experimental respiratory anthrax infection in the common marmoset (Callithrix jacchus). International journal of experimental pathology 89(3):171-179.

Li Y, Ropp SL, Zhao H, Damon IK, Esposito JJ. 2007. Orthopoxvirus pan-genomic DNA assay. Journal of virological methods 141(2):154-165.

Lin CL, Chung CS, Heine HG, Chang W. 2000. Vaccinia virus envelope H3L protein binds to cell surface heparan sulfate and is important for intracellular mature virion morphogenesis and virus infection in vitro and in vivo. Journal of virology 74 (7):3353-3365.

Loparev VN, Parsons JM, Knight JC, Panus JF, Ray CA, Buller RM, Pickup DJ, Esposito JJ. 1998. A third distinct tumor necrosis factor receptor of orthopoxviruses. Proceedings of the National Academy of Sciences of the United States of America 95(7):37863791.

Lu B, Yu W, Huang X, Wang H, Liu L, Chen Z. 2011. Mucosal immunization induces a higher level of lasting neutralizing antibody response in mice by a replicationcompetent smallpox vaccine: vaccinia Tiantan strain. Journal of biomedicine \& biotechnology 2011:970424.

Lustig S, Fogg C, Whitbeck JC, Eisenberg RJ, Cohen GH, Moss B. 2005. Combinations of polyclonal or monoclonal antibodies to proteins of the outer membranes of the two infectious forms of vaccinia virus protect mice against a lethal respiratory challenge. Journal of virology 79(21):13454-13462.

Lustig S, Fogg C, Whitbeck JC, Moss B. 2004. Synergistic neutralizing activities of antibodies to outer membrane proteins of the two infectious forms of vaccinia virus in the presence of complement. Virology 328(1):30-35.

Lycke N. 2012. Recent progress in mucosal vaccine development: potential and limitations. Nature reviews Immunology 12(8):592-605.

Lyons AS, Petrucelli RJ. 1987. Medicine-An Illustrated History. New York: Abradale Press, Harry N Abrams Inc.

Mac CF, Mc Pherson C, Johnstone DF. 1950. Laboratory investigation of smallpox patients with particular reference to infectivity in the early stages. Lancet 2 6637:514-517.

Mack TM, Noble J, Jr., Thomas DB. 1972. A prospective study of serum antibody and protection against smallpox. The American journal of tropical medicine and hygiene 21(2):214-218. 
Marsden JP. 1936. A critical review of the clinical features of 13,686 cases of smallpox (variola

minor), London County Council. Bulletin of hygiene 23:735-746.

Marshall JW, Ridley RM. 2003. Assessment of cognitive and motor deficits in a marmoset model of stroke. ILAR journal 44(2):153-160.

Martin DB. 2002. The cause of death in smallpox: an examination of the pathology record. Military medicine 167(7):546-551.

Martina BE, van Doornum G, Dorrestein GM, Niesters HG, Stittelaar KJ, Wolters MA, van Bolhuis HG, Osterhaus AD. 2006. Cowpox virus transmission from rats to monkeys, the Netherlands. Emerging infectious diseases 12(6):1005-1007.

Matho MH, Maybeno M, Benhnia MR, Becker D, Meng X, Xiang Y, Crotty S, Peters B, Zajonc DM. 2012. Structural and biochemical characterization of the vaccinia virus envelope protein D8 and its recognition by the antibody LA5. Journal of virology 86(15):8050-8058.

Matz-Rensing K, Ellerbrok H, Ehlers B, Pauli G, Floto A, Alex M, Czerny CP, Kaup FJ. 2006. Fatal poxvirus outbreak in a colony of New World monkeys. Vet Pathol 43(2):212-218.

Mätz-Rensing K, Stahl-Hennig C, Kramski M, Pauli G, Ellerbrok H, Kaup FJ. 2012. The pathology of experimental poxvirus infection in common CM (Callithrix jacchus): further characterization of a new primate model for orthopoxvirus infections. Journal of comparative pathology 146(2-3):230-242.

Mayr A, Danner K. 1978. Vaccination against pox diseases under immunosuppressive conditions. Developments in biological standardization 41:225-234.

Mayr A, Danner K. 1979. [Significance of animal pox for man following elimination of compulsory vaccination against smallpox]. Berliner und Munchener tierarztliche Wochenschrift 92(13):251-256.

Mayr A, Stickl H, Muller HK, Danner K, Singer H. 1978. [The smallpox vaccination strain MVA: marker, genetic structure, experience gained with the parenteral vaccination and behavior in organisms with a debilitated defence mechanism (author's transl)]. Zentralblatt fur Bakteriologie, Parasitenkunde, Infektionskrankheiten und Hygiene Erste Abteilung Originale Reihe B: Hygiene, Betriebshygiene, praventive Medizin 167(5-6):375-390.

Mc CK, Downie AW, Bradley WH. 1958. The antibody response in man following infection with viruses of the pox group. II. Antibody response following vaccination. The Journal of hygiene 56(4):466-478.

McClain DJ, Harrison S, Yeager CL, Cruz J, Ennis FA, Gibbs P, Wright MS, Summers PL, Arthur JD, Graham JA. 1997. Immunologic responses to vaccinia vaccines administered by different parenteral routes. The Journal of infectious diseases 175(4):756-763.

McCollum AM, Damon IK. 2014. Human monkeypox. Clinical infectious diseases : an official publication of the Infectious Diseases Society of America 58(2):260-267.

McCollum AM, Nakazawa Y, Ndongala GM, Pukuta E, Karhemere S, Lushima RS, Ilunga BK, Kabamba J, Wilkins K, Gao J and others. 2015. Human Monkeypox in the Kivus, a Conflict Region of the Democratic Republic of the Congo. The American journal of tropical medicine and hygiene 93(4):718-721.

McFadden G, Murphy PM. 2000. Host-related immunomodulators encoded by poxviruses and herpesviruses. Current opinion in microbiology 3(4):371-378.

Meseda CA, Garcia AD, Kumar A, Mayer AE, Manischewitz J, King LR, Golding H, Merchlinsky M, Weir JP. 2005. Enhanced immunogenicity and protective effect 
conferred by vaccination with combinations of modified vaccinia virus Ankara and licensed smallpox vaccine Dryvax in a mouse model. Virology 339(2):164-175.

Meyer H, Sutter G, Mayr A. 1991. Mapping of deletions in the genome of the highly attenuated vaccinia virus MVA and their influence on virulence. The Journal of general virology 72 ( Pt 5):1031-1038.

Moise L, Buller RM, Schriewer J, Lee J, Frey SE, Weiner DB, Martin W, De Groot AS. 2011. VennVax, a DNA-prime, peptide-boost multi-T-cell epitope poxvirus vaccine, induces protective immunity against vaccinia infection by $\mathrm{T}$ cell response alone. Vaccine 29(3):501-511.

Moore ZS, Seward JF, Lane JM. 2006. Smallpox. Lancet (London, England) 367(9508):425435.

Morand A, Delaigue S, Morand JJ. 2017. Review of poxvirus: emergence of monkeypox. Medecine et sante tropicales 27(1):29-39.

Morgan C. 1976. Vaccinia virus reexamined: development and release. Virology 73(1):43-58.

Morgan C, Ellison SA, Rose HM, Moore DH. 1954. Structure and development of viruses observed in the electron microscope. II. Vaccinia and fowl pox viruses. The Journal of experimental medicine 100(3):301-310.

Morgan C, Wyckoff RW. 1950. The electron microscopy of fowl pox virus within the chorioallantoic membrane. Journal of immunology 65(2):285-295.

Moss B. 2011. Smallpox vaccines: targets of protective immunity. Immunological reviews 239(1):8-26.

Moss B. 2013. Poxvirus DNA replication. Cold Spring Harb Persp Biol 5.

Moss B. 2015. Poxvirus membrane biogenesis. Virology 479-480:619-626.

Moss B, Henderson DA. 1999. Smallpox and Vaccinia, Plotkin, S.A., Orenstein, W.A., Vaccines third, W.B. Saunders Company, Philadelphia. .

Moss B, Rosenblum EN. 1973. Letter: Protein cleavage and poxvirus morphogenesis: tryptic peptide analysis of core precursors accumulated by blocking assembly with rifampicin. Journal of molecular biology 81(2):267-269.

Mota BE, Gallardo-Romero N, Trindade G, Keckler MS, Karem K, Carroll D, Campos MA, Vieira LQ, da Fonseca FG, Ferreira PC and others. 2011. Adverse events post smallpox-vaccination: insights from tail scarification infection in mice with Vaccinia virus. PLoS One 6(4):e18924.

Mucker EM, Chapman J, Huzella LM, Huggins JW, Shamblin J, Robinson CG, Hensley LE. 2015. Susceptibility of CM (Callithrix jacchus) to Monkeypox Virus: A Low Dose Prospective Model for Monkeypox and Smallpox Disease. PLoS One 10(7):e0131742.

Mucker EM, Goff AJ, Shamblin JD, Grosenbach DW, Damon IK, Mehal JM, Holman RC, Carroll D, Gallardo N, Olson VA and others. 2013. Efficacy of tecovirimat (ST-246) in nonhuman primates infected with variola virus (Smallpox). Antimicrobial agents and chemotherapy 57(12):6246-6253.

Munoz FM. 2014a. Manson's Tropical Infectious Diseases (32).

Munoz FM. 2014b. Viral Exanthems. Manson's Tropical Infectious Diseases 23.

Nagel A, Mobs C, Raifer H, Wiendl H, Hertl M, Eming R. 2014. CD3-positive B cells: a storage-dependent phenomenon. PLoS One 9(10):e110138.

Nalca A, Rimoin AW, Bavari S, Whitehouse CA. 2005. Reemergence of monkeypox: prevalence, diagnostics, and countermeasures. Clinical infectious diseases : an official publication of the Infectious Diseases Society of America 41(12):1765-1771.

Needham J. 1980. China and the origins of immunology. Eastern horizon 19(1):6-12.

Nelson GE, Wagenaar TR, Moss B. 2008. A conserved sequence within the H2 subunit of the vaccinia virus entry/fusion complex is important for interaction with the A28 subunit and infectivity. Journal of virology 82(13):6244-6250. 
Neubert R, Helge H, Neubert D. 1995. Proliferative capacity of marmoset lymphocytes after tetanus vaccination and lack of 2,3,7,8-tetrachlorodibenzo-p-dioxin to reduce a booster effect. Life sciences 56(6):437-444.

Neumann B, Klippert A, Raue K, Sopper S, Stahl-Hennig C. 2015. Characterization of B and plasma cells in blood, bone marrow, and secondary lymphoid organs of rhesus macaques by multicolor flow cytometry. Journal of leukocyte biology 97(1):19-30.

Oda KI, Joklik WK. 1967. Hybridization and sedimentation studies on "early" and "late" vaccinia messenger RNA. Journal of molecular biology 27(3):395-419.

Orba Y, Sasaki M, Yamaguchi H, Ishii A, Thomas Y, Ogawa H, Hang'ombe BM, Mweene AS, Morikawa S, Saijo M and others. 2015. Orthopoxvirus infection among wildlife in Zambia. The Journal of general virology 96(Pt 2):390-394.

Orr N, Forman M, Marcus H, Lustig S, Paran N, Grotto I, Klement E, Yehezkelli Y, Robin G, Reuveny $S$ and others. 2004. Clinical and immune responses after revaccination of israeli adults with the Lister strain of vaccinia virus. The Journal of infectious diseases 190(7):1295-1302.

Paluku KM, Szczeniowski MV. 1988. Human monkeypox: disease pattern, incidence and attack rates in a rural area of northern Zaire. Trop Geogr Med 40(2):73-83.

Paran N, Sutter G. 2009. Smallpox vaccines: New formulations and revised strategies for vaccination. Human vaccines 5(12):824-831.

Parker S, Nuara A, Buller RM, Schultz DA. 2007. Human monkeypox: an emerging zoonotic disease. Future microbiology 2(1):17-34.

Patel DD, Pickup DJ, Joklik WK. 1986. Isolation of cowpox virus A-type inclusions and characterization of their major protein component. Virology 149 (2):174-189.

Paul WE. 2008. Fundamental Immunology. Wolters Kluwer Health, Lippincott Williams \& Wilkins:1236.

Perdiguero B, Esteban M. 2009. The interferon system and vaccinia virus evasion mechanisms. Journal of interferon \& cytokine research : the official journal of the International Society for Interferon and Cytokine Research 29(9):581-598.

Pereira Oliveira G, Tavares Silva Fernandes A, Lopes de Assis F, Augusto Alves P, Moreira Franco Luiz AP, Barcelos Figueiredo L, ..., Geessien Kroon E. 2014. Intrafamilial Transmission of Vaccinia virus during a Bovine Vaccinia Outbreak in Brazil: A New Insight in Viral Transmission Chain. . The American journal of tropical medicine and hygiene 90(6):1021-1023.

Pether JV, Trevains PH, Harrison SR, Baxby D, Bennett M, Gibb AP. 1986. Cowpox from cat to man. Lancet (London, England) 1(8471):38-39.

Pilaski J, Rosen A, Darai G. 1986

Comparative analysis of the genomes of orthopoxviruses isolated from elephant, rhinoceros, and okapi by restriction enzymes. . Brief report, Arch Virol 88 (1-2):135-142.

Puissant B, Combadiere B. 2006. Keeping the memory of smallpox virus. Cellular and molecular life sciences : CMLS 63(19-20):2249-2259.

Pulford DJ, Gates A, Bridge SH, Robinson JH, Ulaeto D. 2004. Differential efficacy of vaccinia virus envelope proteins administered by DNA immunisation in protection of BALB/c mice from a lethal intranasal poxvirus challenge. Vaccine 22(25-26):33583366.

Putz MM, Midgley CM, Law M, Smith GL. 2006. Quantification of antibody responses against multiple antigens of the two infectious forms of Vaccinia virus provides a benchmark for smallpox vaccination. Nature medicine 12(11):1310-1315.

Radetsky M. 1999. Smallpox: a history of its rise and fall. The Pediatric infectious disease journal 18(2):85-93. 
Rao AR, Prahlad I, Swaminathan M. 1960. A study of 1,000 Cases of Smallpox. Journal of the Indian Medical Association 196035 (7):296-307

Reardon S. 2014. 'Forgotten' NIH smallpox virus languishes on death row. Nature 514(7524):544.

Reed KD. 2004. Monkeypox, Marshfield Clinic and the Internet: leveraging information technology for public health. Clinical medicine \& research 2(1):1-3.

Ricketts TF. 1908. The diagnosis of smallpox. London: Cassell.

Riedel S. 2005. Edward Jenner and the history of smallpox and vaccination. Proceedings 18(1):21-25.

Rimoin AW, Mulembakani PM, Johnston SC, Lloyd Smith JO, Kisalu NK, Kinkela TL, Blumberg S, Thomassen HA, Pike BL, Fair JN and others. 2010. Major increase in human monkeypox incidence 30 years after smallpox vaccination campaigns cease in the Democratic Republic of Congo. Proceedings of the National Academy of Sciences of the United States of America 107(37):16262-16267.

Rock MT, Yoder SM, Wright PF, Talbot TR, Edwards KM, Crowe JE, Jr. 2005. Differential regulation of granzyme and perforin in effector and memory $\mathrm{T}$ cells following smallpox immunization. Journal of immunology 174(6):3757-3764.

Rodriguez JR, Risco C, Carrascosa JL, Esteban M, Rodriguez D. 1997. Characterization of early stages in vaccinia virus membrane biogenesis: implications of the 21-kilodalton protein and a newly identified 15-kilodalton envelope protein. Journal of virology 71(3):1821-1833.

Rodriguez JR, Risco C, Carrascosa JL, Esteban M, Rodriguez D. 1998. Vaccinia virus 15kilodalton (A14L) protein is essential for assembly and attachment of viral crescents to virosomes. J Virol 72(2):1287-1296.

Roper RL, Payne LG, Moss B. 1996. Extracellular vaccinia virus envelope glycoprotein encoded by the A33R gene. Journal of virology 70(6):3753-3762.

Rowe MJ, Turman AB, Murray GM, Zhang HQ. 1996. Parallel organization of somatosensory cortical areas I and II for tactile processing. Clinical and experimental pharmacology \& physiology 23(10-11):931-938.

Safronetz D, Geisbert TW, Feldmann H. 2013. Animal models for highly pathogenic emerging viruses. Current opinion in virology 3(2):205-209.

Sakhatskyy P, Wang S, Chou TH, Lu S. 2006. Immunogenicity and protection efficacy of monovalent and polyvalent poxvirus vaccines that include the D8 antigen. Virology 355(2):164-174.

Sakhatskyy P, Wang S, Zhang C, Chou TH, Kishko M, Lu S. 2008. Immunogenicity and protection efficacy of subunit-based smallpox vaccines using variola major antigens. Virology 371(1):98-107.

Sale TA, Melski JW, Stratman EJ. 2006. Monkeypox: an epidemiologic and clinical comparison of African and US disease. Journal of the American Academy of Dermatology 55(3):478-481.

Sanchez-Puig JM, Sanchez L, Roy G, Blasco R. 2004. Susceptibility of different leukocyte cell types to Vaccinia virus infection. Virology journal 1:10.

Sarkar JK, Mitra AC, Mukherjee MK. 1975. The minimum protective level of antibodies in smallpox. Bulletin of the World Health Organization 52(3):307-311.

Schmelz M, Sodeik B, Ericsson M, Wolffe EJ, Shida H, Hiller G, Griffiths G. 1994. Assembly of vaccinia virus: the second wrapping cisterna is derived from the trans Golgi network. Journal of virology 68(1):130-147.

Schmitt A, Gan L, Stahl-Hennig C, Shi T, Ellerbrok H, Kaup F-J, Mätz-Rensing K. 2017. Dynamics of pathological findings during experimental calpox virus infection of common CM (Callithrix jacchus). 2017. Viruses 9, 363; doi:10.3390/v9120363. 
Schmitt A, Mätz-Rensing K, Kaup F-J. 2014. Non-Human Primate Models of Orthopoxvirus Infections veterinary sciences (1 ):40-62.

Schmitt AE. 2015. Untersuchungen zur experimentellen Infektion von CM (Callithrix jacchus) mit dem Calpoxvirus. Dissertation.

Schneider CA, Rasband WS, Eliceiri KW. 2012. NIH Image to ImageJ: 25 years of image analysis. Nature methods 9(7):671-675.

Schuh E, Berer K, Mulazzani M. 2016. Features of Human CD3+CD20+ T Cells. 197(4):1111-1117.

Schupp CJ, Nitsche A, Bock-Hensley O, Bohm S, Flechtenmacher C, Kurth A, Saenger K, Hoferer M, Kusters U, Gunther P and others. 2011. A 14-year-old girl with a vesicle on her finger and lymphadenitis. Journal of clinical virology : the official publication of the Pan American Society for Clinical Virology 50(1):1-3.

Seet BT, Johnston JB, Brunetti CR, Barrett JW, Everett H, Cameron C, Sypula J, Nazarian SH, Lucas A, McFadden G. 2003. Poxviruses and immune evasion. Annual review of immunology 21:377-423.

Senkevich TG, Moss B. 2005. Vaccinia virus H2 protein is an essential component of a complex involved in virus entry and cell-cell fusion. Journal of virology 79(8):47444754.

Sette A, Grey H, Oseroff C, Peters B, Moutaftsi M, Crotty S, Assarsson E, Greenbaum J, Kim Y, Kolla R and others. 2009. Definition of epitopes and antigens recognized by vaccinia specific immune responses: their conservation in variola virus sequences, and use as a model system to study complex pathogens. Vaccine 27 Suppl 6:G21-26.

Shchelkunov SN. 2013. An increasing danger of zoonotic orthopoxvirus infections. PLoS pathogens 9(12):e1003756.

Shchelkunov SN, Safronov PF, Totmenin AV, Petrov NA, Ryazankina OI, Gutorov VV, Kotwal GJ. 1998. The genomic sequence analysis of the left and right species-specific terminal region of a cowpox virus strain reveals unique sequences and a cluster of intact ORFs for immunomodulatory and host range proteins. Virology 243(2):432460.

Shchelkunov SN, Totmenin AV, Loparev VN, Safronov PF, Gutorov VV, Chizhikov VE, Knight JC, Parsons JM, Massung RF, Esposito JJ. 2000. Alastrim smallpox variola minor virus genome DNA sequences. Virology 266(2):361-386.

Simcock PR, Noble JL, Tullo AB, Morris DJ, Morgan-Capner P. 1993. Cowpox virus. The British journal of ophthalmology 77(6):394.

Singh RK, Balamurugan V, Hosamani M, Kallesh DJ, Bhanuprakash V. 2008. Sequence analysis of C18L gene of buffalopox virus: PCR strategy for specific detection and differentiation of buffalopox from orthopoxviruses. Journal of virological methods 154(1-2):146-153.

Singh RK, Hosamani M, Balamurugan V, Bhanuprakash V, Rasool TJ, Yadav MP. 2007. Buffalopox: an emerging and re-emerging zoonosis. Animal health research reviews 8(1):105-114.

Singh RK, Hosamani M, Balamurugan V, Satheesh CC, Shingal KR, Tatwarti SB, Bambal RG, Ramteke V, Yadav MP. 2006. An outbreak of buffalopox in buffalo (Bubalus bubalis) dairy herds in Aurangabad, India. Revue scientifique et technique (International Office of Epizootics) 25(3):981-987.

Smith GL, Law M. 2004. The exit of vaccinia virus from infected cells. Virus research 106( 2):189-197.

Smith GL, McFadden G. 2002. Smallpox: anything to declare? Nature reviews Immunology 2(7):521-527. 
Smith GL, Vanderplasschen A, Law M. 2002. The formation and function of extracellular enveloped vaccinia virus. The Journal of general virology 83(Pt 12):2915-2931.

Smith SA, Kotwal GJ. 2002. Immune response to poxvirus infections in various animals. Critical reviews in microbiology 28(3):149-185.

Smith VP, Bryant NA, Alcami A. 2000. Ectromelia, vaccinia and cowpox viruses encode secreted interleukin-18-binding proteins. The Journal of general virology $81(\mathrm{Pt}$ 5):1223-1230.

Song H, Janosko K, Johnson RF, Qin J, Josleyn N, Jett C, Byrum R, St Claire M, Dyall J, Blaney JE and others. 2013a. Poxvirus Antigen Staining of Immune Cells as a Biomarker to Predict Disease Outcome in Monkeypox and Cowpox Virus Infection in Non-Human Primates. PLoS ONE 8(4).

Song H, Janosko K, Johnson RF, Qin J, Josleyn N, Jett C, Byrum R, St Claire M, Dyall J, Blaney JE and others. 2013b. Poxvirus antigen staining of immune cells as a biomarker to predict disease outcome in monkeypox and cowpox virus infection in non-human primates. PLoS One 8(4):e60533.

Staib C, Suezer Y, Kisling S, Kalinke U, Sutter G. 2006. Short-term, but not post-exposure, protection against lethal orthopoxvirus challenge after immunization with modified vaccinia virus Ankara. The Journal of general virology 87(Pt 10):2917-2921.

Stanford MM, McFadden G, Karupiah G, Chaudhri G. 2007. Immunopathogenesis of poxvirus infections: forecasting the impending storm. Immunology and cell biology 85(2):93-102.

Stittelaar KJ, Kuiken T, de Swart RL, van Amerongen G, Vos HW, Niesters HG, van Schalkwijk P, van der Kwast T, Wyatt LS, Moss B and others. 2001. Safety of modified vaccinia virus Ankara (MVA) in immune-suppressed macaques. Vaccine 19(27):3700-3709.

Stittelaar KJ, Neyts J, Naesens L, van Amerongen G, van Lavieren RF, Holy A, De Clercq E, Niesters HG, Fries E, Maas C and others. 2006. Antiviral treatment is more effective than smallpox vaccination upon lethal monkeypox virus infection. Nature 439(7077):745-748.

Stittelaar KJ, van Amerongen G, Kondova I, Kuiken T, van Lavieren RF, Pistoor FH, Niesters HG, van Doornum G, van der Zeijst BA, Mateo L and others. 2005. Modified vaccinia virus Ankara protects macaques against respiratory challenge with monkeypox virus. Journal of virology 79(12):7845-7851.

t Hart BA, van Meurs M, Brok HP, Massacesi L, Bauer J, Boon L, Bontrop RE, Laman JD. 2000. A new primate model for multiple sclerosis in the common marmoset. Immunology today 21(6):290-297.

Taub DD, Ershler WB, Janowski M, Artz A, Key ML, McKelvey J, Muller D, Moss B, Ferrucci L, Duffey PL and others. 2008. Immunity from smallpox vaccine persists for decades: a longitudinal study. The American journal of medicine 121(12):1058-1064.

Taylor JM, Barry M. 2006. Near death experiences: poxvirus regulation of apoptotic death. Virology 344(1):139-150.

Thomassen HA, Fuller T, Asefi-Najafabady S, Shiplacoff JA, Mulembakani PM, Blumberg S, Johnston SC, Kisalu NK, Kinkela TL, Fair JN and others. 2013. Pathogen-host associations and predicted range shifts of human monkeypox in response to climate change in central Africa. PLoS One 8(7):e66071.

Tooze J, Hollinshead M, Reis B, Radsak K, Kern H. 1993. Progeny vaccinia and human cytomegalovirus particles utilize early endosomal cisternae for their envelopes. European journal of cell biology 60(1):163-178. 
Townsley AC, Senkevich TG, Moss B. 2005. The product of the vaccinia virus L5R gene is a fourth membrane protein encoded by all poxviruses that is required for cell entry and cell-cell fusion. Journal of virology 79(17):10988-10998.

Tucker JB. 2011. Breaking the deadlock over destruction of the smallpox virus stocks. Biosecurity and bioterrorism : biodefense strategy, practice, and science 9(1):55-67.

Uccelli A, Oksenberg JR, Jeong MC, Genain CP, Rombos T, Jaeger EE, Giunti D, Lanchbury JS, Hauser SL. 1997. Characterization of the TCRB chain repertoire in the New World monkey Callithrix jacchus. Journal of immunology 158(3):1201-1207.

Uebler N. 2008. A phase I study to evaluate take rate, immunogenicity and safety of the 2nd generation smallpox vaccine Elstree-BN, Bavarian Nordic, GMBH. Martinsried, Germany, 10/08/2008.

Upton C, Slack S, Hunter AL, Ehlers A, Roper RL. 2003. Poxvirus Orthologous Clusters: toward Defining the Minimum Essential Poxvirus Genome. Journal of virology.

van Vliet SA, Vanwersch RA, Jongsma MJ, Olivier B, Philippens IH. 2008. Therapeutic effects of Delta9-THC and modafinil in a marmoset Parkinson model. European neuropsychopharmacology : the journal of the European College of Neuropsychopharmacology 18(5):383-389.

Vanderplasschen A, Mathew E, Hollinshead M, Sim RB, Smith GL. 1998. Extracellular enveloped vaccinia virus is resistant to complement because of incorporation of host complement control proteins into its envelope. Proceedings of the National Academy of Sciences of the United States of America 95(13):7544-7549.

Vanderplasschen A, Smith GL. 1997. A novel virus binding assay using confocal microscopy: demonstration that the intracellular and extracellular vaccinia virions bind to different cellular receptors. Journal of virology 71(5):4032-4041.

Vazquez MI, Rivas G, Cregut D, Serrano L, Esteban M. 1998. The vaccinia virus 14kilodalton (A27L) fusion protein forms a triple coiled-coil structure and interacts with the 21-kilodalton (A17L) virus membrane protein through a C-terminal alpha-helix. Journal of virology 72 (12):10126-10137.

Velasquez LS, Shira S, Berta AN, Kilbourne J, Medi BM, Tizard I, Ni Y, Arntzen CJ, HerbstKralovetz MM. 2011. Intranasal delivery of Norwalk virus-like particles formulated in an in situ gelling, dry powder vaccine. Vaccine 29(32):5221-5231.

Verardi PH, Titong A, Hagen CJ. 2012. A vaccinia virus renaissance: new vaccine and immunotherapeutic uses after smallpox eradication. Human vaccines \& immunotherapeutics 8(7):961-970.

Viner KM, Isaacs SN. 2005. Activity of vaccinia virus-neutralizing antibody in the sera of smallpox vaccinees. Microbes and infection 7(4):579-583.

Vollmar J, Arndtz N, Eckl KM, Thomsen T, Petzold B, Mateo L, Schlereth B, Handley A, King L, Hulsemann V and others. 2006. Safety and immunogenicity of IMVAMUNE, a promising candidate as a third generation smallpox vaccine. Vaccine 24(12):20652070.

von Krempelhuber A, Vollmar J, Pokorny R, Rapp P, Wulff N, Petzold B, Handley A, Mateo L, Siersbol H, Kollaritsch H and others. 2010. A randomized, double-blind, dosefinding Phase II study to evaluate immunogenicity and safety of the third generation smallpox vaccine candidate IMVAMUNE. Vaccine 28(5):1209-1216.

Vorou RM, Papavassiliou VG, Pierroutsakos IN. 2008. Cowpox virus infection: an emerging health threat. Current opinion in infectious diseases 21(2):153-156.

Wallengren K, Risco C, Krijnse-Locker J, Esteban M, Rodriguez D. 2001. The A17L gene product of vaccinia virus is exposed on the surface of IMV. Virology 290(1):143-152.

Walsh SR, Wilck MB, Dominguez DJ, Zablowsky E, Bajimaya S, Gagne LS, Verrill KA, Kleinjan JA, Patel A, Zhang Y and others. 2013. Safety and immunogenicity of 
modified vaccinia Ankara in hematopoietic stem cell transplant recipients: a randomized, controlled trial. The Journal of infectious diseases 207(12):1888-1897.

Wei CM, Moss B. 1975. Methylated nucleotides block 5'-terminus of vaccinia virus messenger RNA. Proceedings of the National Academy of Sciences of the United States of America 72(1):318-322.

Wenzel RP. 2002. Recognizing the real threat of biological terror. Transactions of the American Clinical and Climatological Association 113:42-55.

WHO. 1980. The global eradication of smallpox. Final report of the global commission for the certification of smallpox eradication. History of International Public Health No4, Geneva, World Health Organization, No4

Willemse A, Egberink HF. 1985. Transmission of cowpox virus infection from domestic cat to man. Lancet (London, England) 1(8444):1515.

Wolffe EJ, Vijaya S, Moss B. 1995. A myristylated membrane protein encoded by the vaccinia virus L1R open reading frame is the target of potent neutralizing monoclonal antibodies. Virology 211(1):53-63.

Wolfs TF, Wagenaar JA, Niesters HG, Osterhaus AD. 2002. Rat-to-human transmission of Cowpox infection. Emerging infectious diseases 8(12):1495-1496.

Woollard DJ, Haqshenas G, Dong X, Pratt BF, Kent SJ, Gowans EJ. 2008. Virus-specific Tcell immunity correlates with control of GB virus B infection in CM. Journal of virology 82(6):3054-3060.

Xiao Y, Aldaz-Carroll L, Ortiz AM, Whitbeck JC, Alexander E, Lou H, Davis HL, Braciale TJ, Eisenberg RJ, Cohen GH and others. 2007. A protein-based smallpox vaccine protects mice from vaccinia and ectromelia virus challenges when given as a prime and single boost. Vaccine 25(7):1214-1224.

Xu D, Regner M, Smith D, Ruby J, Johnstone R, Mullbacher A. 2004a. The multidrug resistance gene mdrla influences resistance to ectromelia virus infection by mechanisms other than conventional immunity. Immunology and cell biology 82(5):462-470.

Xu R, Johnson AJ, Liggitt D, Bevan MJ. 2004b. Cellular and humoral immunity against vaccinia virus infection of mice. Journal of immunology 172(10):6265-6271.

Xu Y, Yuen PW, Lam JK. 2014. Intranasal DNA Vaccine for Protection against Respiratory Infectious Diseases: The Delivery Perspectives. Pharmaceutics 6(3):378-415.

Yadav S, Hosamani M, Balamurugan V, Bhanuprakash V, Singh RK. 2010. Partial genetic characterization of viruses isolated from pox-like infection in cattle and buffaloes: evidence of buffalo pox virus circulation in Indian cows. Archives of virology 155(2):255-261.

Yu W, Fang Q, Zhu W, Wang H, Tien P, Zhang L, Chen Z. 2010. One time intranasal vaccination with a modified vaccinia Tiantan strain MVTT(ZCI) protects animals against pathogenic viral challenge. Vaccine 28(9):2088-2096.

Zaucha GM, Jahrling PB, Geisbert TW, Swearengen JR, Hensley L. 2001. The pathology of experimental aerosolized monkeypox virus infection in cynomolgus monkeys (Macaca fascicularis). Laboratory investigation; a journal of technical methods and pathology 81(12):1581-1600.

Zhang HQ, Murray GM, Turman AB, Mackie PD, Coleman GT, Rowe MJ. 1996. Parallel processing in cerebral cortex of the marmoset monkey: effect of reversible SI inactivation on tactile responses in SII. Journal of neurophysiology 76(6):3633-3655.

Zhang Q, Tian M, Feng Y, Zhao K, Xu J, Liu Y, Shao Y. 2013. Genomic sequence and virulence of clonal isolates of vaccinia virus Tiantan, the Chinese smallpox vaccine strain. PLoS One 8(4):e60557. 
Zhu W, Fang Q, Zhuang K, Wang H, Yu W, Zhou J, Liu L, Tien P, Zhang L, Chen Z. 2007. The attenuation of vaccinia Tian Tan strain by the removal of the viral M1L-K2L genes. Journal of virological methods 144(1-2):17-26.

Zielinski RJ, Smedley JV, Perera PY, Silvera PM, Waldmann TA, Capala J, Perera LP. 2010. Smallpox vaccine with integrated IL-15 demonstrates enhanced in vivo viral clearance in immunodeficient mice and confers long term protection against a lethal monkeypox challenge in cynomolgus monkeys. Vaccine 28(43):7081-7091.

\subsection{Internet cited}

http://www.dpz.eu/de/abteilung/primatenhaltung/primaten-am-dpz/weissbueschelaffe.html (24.11.2017) 


\section{A Appendix}

\section{A.1 Abbreviations}

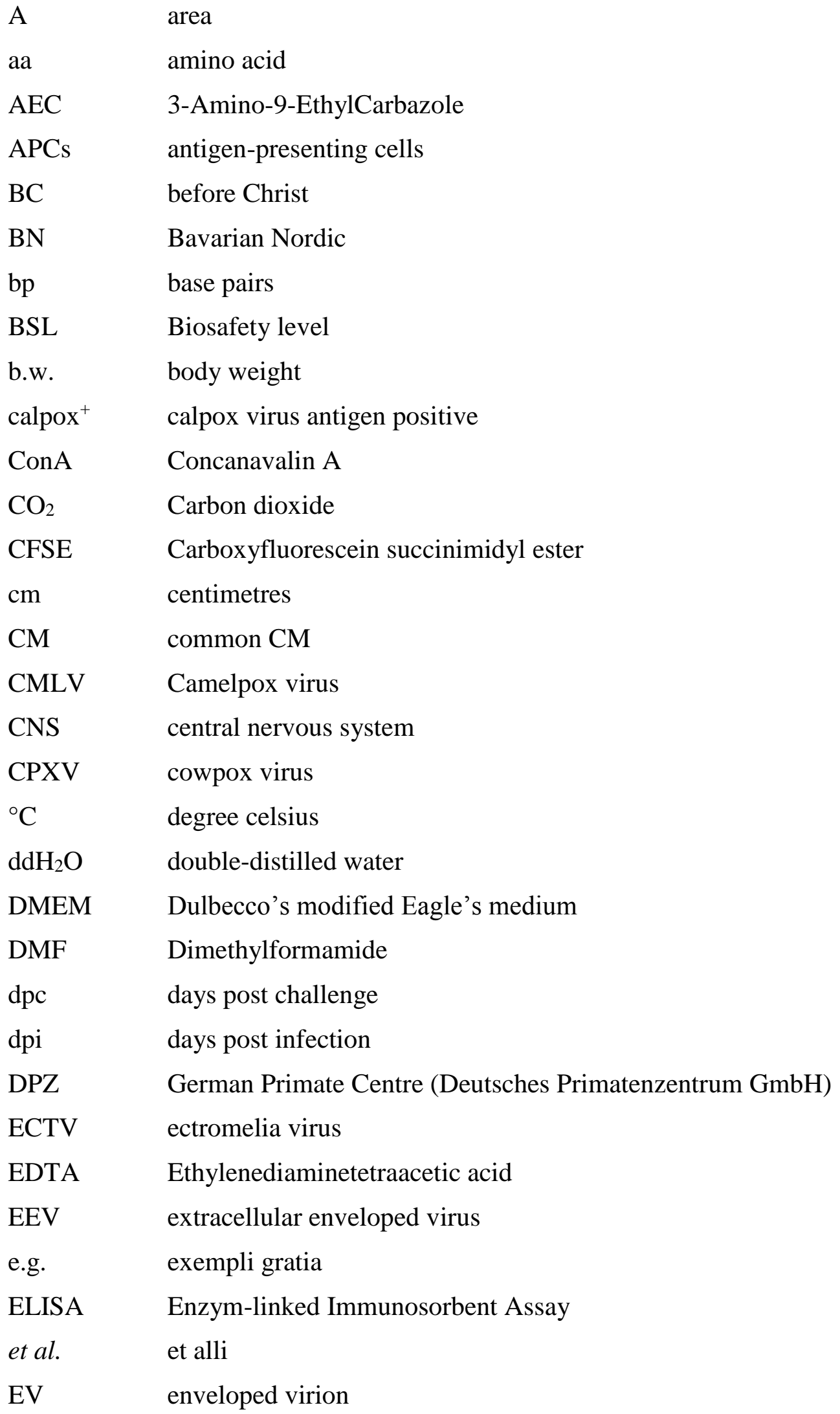




\begin{tabular}{|c|c|}
\hline FA & formaldehyde \\
\hline FAM & 6-FAM (6-Carboxyfluorescein) \\
\hline FCS & fetal calf serum \\
\hline fmol & femtomole \\
\hline $\mathrm{h}$ & hour \\
\hline HLA-DR & human leukocyte antigen - antigen D related \\
\hline $\mathrm{H}_{2} \mathrm{O}_{2}$ & Hydrogen peroxide \\
\hline i.d. & intradermally \\
\hline i.e. & id est \\
\hline IEV & intracellular enveloped virus \\
\hline i.m. & intramuscularly \\
\hline IMV & intracellular mature virus \\
\hline i.n. & intranasally \\
\hline i.p. & intraperitoneal \\
\hline i.v. & intravenous \\
\hline $\mathrm{kb}$ & kilobase \\
\hline $\mathrm{KCl}$ & potassium chloride \\
\hline $\mathrm{kDa}$ & kilodalton \\
\hline $\mathrm{kg}$ & kilograms \\
\hline LE & Lister Elstree \\
\hline $\mathrm{LN}$ & lymph node \\
\hline $\mathrm{mM}$ & Millimol \\
\hline M & molar mass \\
\hline mAbs & monoclonal antibodies \\
\hline $\min$ & minutes \\
\hline MOI & Multiplicity of infection \\
\hline MV & mature virion \\
\hline MVA & Modified vaccinia virus Ankara \\
\hline MVTT & Modified vaccinia virus Tiantan \\
\hline $\mathrm{mDCs}$ & myeloid dendritic cells \\
\hline $\mathrm{mg}$ & milligrams \\
\hline $\mathrm{MID}_{50}$ & median monkey infectious doses \\
\hline $\mathrm{ml}$ & millilitres \\
\hline MPXV & monkeypox virus \\
\hline MQs & macrophages \\
\hline
\end{tabular}




\begin{tabular}{|c|c|}
\hline $\mathrm{nAb}$ & neutralizing antibody \\
\hline $\mathrm{NaCl}$ & sodium chloride \\
\hline NALT & nasal-associated lymphoid tissue \\
\hline $\mathrm{NaOAc}$ & sodium acetate \\
\hline $\mathrm{nm}$ & nanometers \\
\hline NHP & non-human primate \\
\hline OD & optical density \\
\hline OPXV & orthopoxvirus \\
\hline pfu & plaque forming units \\
\hline pi & post infection \\
\hline p.o. & per oral \\
\hline $\mathrm{P}$ & passage \\
\hline PBMCs & Peripheral blood mononuclear cells \\
\hline PBS & Phosphate-buffered saline \\
\hline $\mathrm{pDCs}$ & plasmacytoid dendritic cells \\
\hline PCR & Polymerase Chain Reaction \\
\hline pfu & plaque forming units \\
\hline $\mathrm{P} / \mathrm{S}$ & Penicillin-Streptomycin \\
\hline $\mathrm{rcf}$ & relative centrifugal force \\
\hline RKI & Robert Koch Institute \\
\hline SD & standard deviation \\
\hline $\sec$ & seconds \\
\hline SSC & side scatter \\
\hline submand. & submandibular \\
\hline TBS & Tris-buffered saline \\
\hline Tм & Trademark \\
\hline $\mathrm{TMB}$ & 3,3',5,5'-Tetramethylbenzidine \\
\hline TMR & 5-TAMRA (5-Carboxytetramethylrhodamine) \\
\hline T-TBS & Tween TBS \\
\hline VACV & vaccinia virus \\
\hline VARV & variola virus \\
\hline vIFN $\gamma \mathrm{R}$ & viral interferon gamma receptor \\
\hline vIL-1R & viral interleukin 1 receptor \\
\hline vTNFR & viral tumor necrosis factor receptor \\
\hline WR & Western Reserve \\
\hline
\end{tabular}




$\begin{array}{ll}\text { wt } & \text { wild type } \\ \mathrm{xg} & \text { times gravity } \\ \% & \text { percent } \\ \mu \mathrm{g} & \text { micrograms } \\ \mu \mathrm{l} & \text { microlitres }\end{array}$

\section{A.2 Tables and Protocols}

Table A.2-1: Göttinger Mixture II (GMII).

\begin{tabular}{l|l}
\hline Ketamine $(100 \mathrm{mg} / \mathrm{kg})$ & $5 \mathrm{ml}$ \\
Xylazine $(10 \%)$ & $1 \mathrm{ml}$ \\
Atropine $(1 \%)$ & $0.1 \mathrm{ml}$ \\
Aqua ad injectionem & $3.9 \mathrm{ml}$ \\
\hline
\end{tabular}

Table A.3-1: Detected replicating calpox virus in Callithrix jacchus individuals' blood for the pathogenesis study by viral plaque assay. Infectious calpox virus was isolation from buffy coat. Plaque assay was processed at 3, 7, 10 and 12 dpi. From day-3-group (animals got the high dose of $3.5 \times 10^{5}$ pfu calpox virus) no replicating virus was detected. First replicating virus $(1 / 3)$ was detected in the low-dose group at 7 days post infection (dpi) (animals were infected $8.3 \times 10^{3}$ pfu calpox virus). Infectious virus particles $[\mathrm{pfu} / \mu \mathrm{l}]$ were detected in all tested animals at $10 \mathrm{dpi}$ and at the last available time point at $12 \mathrm{dpi}$.

\begin{tabular}{l|llllll}
\hline $\begin{array}{l}\text { days post infec- } \\
\text { tion (dpi) }\end{array}$ & \multicolumn{5}{l}{ Study Identification (Group) } \\
\hline \multirow{3}{*}{} & $14325(\mathrm{I})$ & $14484(\mathrm{I})$ & $14991(\mathrm{I})$ & $14702(\mathrm{~V})$ & $15122(\mathrm{~V})$ & $15095(\mathrm{~V})$ \\
7 & 0 & 0 & 0 & n.d. & n.d. & n.d. \\
10 & n.d. & n.d. & n.d. & 0 & 0 & 0.01 \\
12 & n.d. & n.d. & n.d. & 0.20 & 0.06 & 0.16 \\
\hline
\end{tabular}

dpi, days post infection; Infectious virus particles [pfu/ $\mu 1]$, final amount of infectious virus particles [pfu/ $\mu 1]$; n.d., not detected; pfu, plaque forming units.

Table A.3-2: Virus isolation of infectious calpox virus from buffy coat.

\begin{tabular}{|c|c|c|c|c|c|}
\hline Study & $\begin{array}{l}\text { Vac- } \\
\text { cination } \\
\text { group }\end{array}$ & Vaccine & $\begin{array}{l}\text { Study } \\
\text { Identifica- } \\
\text { tion }\end{array}$ & $\begin{array}{l}\text { Plaque assay processed at different } \\
\text { days post infection (dpi) }\end{array}$ & $\begin{array}{l}\text { Infectious virus particles } \\
{[\mathrm{pfu} / \mu \mathrm{l}]}\end{array}$ \\
\hline \multirow{12}{*}{$\begin{array}{l}\text { Vac- } \\
\text { cination } \\
\text { study II }\end{array}$} & \multirow[t]{4}{*}{ III } & MVA & \multirow[t]{4}{*}{14233} & 10 & 0 \\
\hline & & \multirow[t]{3}{*}{ i.m. } & & 12 & 0.067 \\
\hline & & & & 14 & 0.173 \\
\hline & & & & 17 & 3.95 \\
\hline & & & \multirow[t]{8}{*}{14309} & 10 & 0 \\
\hline & & & & 12 & 0 \\
\hline & & & & 14 & 0 \\
\hline & & & & 17 & 0 \\
\hline & & & & 21 & 0 \\
\hline & & & & 24 & 0 \\
\hline & & & & 29 & 0 \\
\hline & & & & 35 & 0 \\
\hline
\end{tabular}




\begin{tabular}{|c|c|c|c|c|}
\hline & & \multirow[t]{5}{*}{14248} & 10 & 0 \\
\hline & & & 12 & 0 \\
\hline & & & 14 & 0 \\
\hline & & & 17 & 0.1 \\
\hline & & & 21 & 1.993 \\
\hline & & \multirow[t]{5}{*}{14372} & 10 & 0 \\
\hline & & & 12 & 0,001 \\
\hline & & & 14 & 0 \\
\hline & & & 17 & 0,166 \\
\hline & & & 21 & 270.667 \\
\hline \multirow[t]{8}{*}{ IV } & MVTT & \multirow[t]{4}{*}{14169} & 10 & 0 \\
\hline & i.n. & & 12 & 0.013 \\
\hline & & & 14 & 0.013 \\
\hline & & & 17 & 0.053 \\
\hline & & \multirow[t]{4}{*}{14603} & 10 & 0 \\
\hline & & & 12 & 0.033 \\
\hline & & & 14 & 0.083 \\
\hline & & & 16 & 0.2 \\
\hline & & \multirow[t]{8}{*}{14409} & 10 & 0 \\
\hline & & & 12 & 0 \\
\hline & & & 14 & 0 \\
\hline & & & 17 & 0 \\
\hline & & & 21 & 0 \\
\hline & & & 24 & 0 \\
\hline & & & 29 & 0 \\
\hline & & & 35 & 0 \\
\hline & & \multirow[t]{8}{*}{14469} & 10 & 0 \\
\hline & & & 12 & 0 \\
\hline & & & 14 & 0 \\
\hline & & & 17 & 0 \\
\hline & & & 21 & 0 \\
\hline & & & 24 & 0 \\
\hline & & & 29 & 0 \\
\hline & & & 35 & 0 \\
\hline \multirow[t]{2}{*}{ control } & PBS i.n. & 14365 & 10 & 1 \\
\hline & & & 12 & 494 \\
\hline \multirow[t]{8}{*}{$\mathrm{V}$} & MVTT & 15098 & 10 & 0 \\
\hline & p.o. & & 12 & 0 \\
\hline & & & 14 & 0 \\
\hline & & & 17 & 0 \\
\hline & & & 21 & 0 \\
\hline & & & 24 & 0 \\
\hline & & & 29 & 0 \\
\hline & & & 35 & 0 \\
\hline
\end{tabular}




\begin{tabular}{|c|c|c|c|c|c|}
\hline & & & 14478 & 10 & 0 \\
\hline & & & & 12 & 0 \\
\hline & & & & 14 & 0 \\
\hline & & & & 17 & 0 \\
\hline & & & & 21 & 0 \\
\hline & & & & 24 & 0 \\
\hline & & & & 29 & 0 \\
\hline & & & & 35 & 0 \\
\hline & & & 14334 & 10 & 0 \\
\hline & & & & 12 & 0 \\
\hline & & & & 14 & 0 \\
\hline & & & & 17 & 0 \\
\hline & & & & 21 & 0 \\
\hline & & & & 24 & 0 \\
\hline & & & & 29 & 0 \\
\hline & & & & 35 & 0 \\
\hline & & & 15100 & 10 & 0 \\
\hline & & & & 12 & 0 \\
\hline & & & & 14 & 0.007 \\
\hline & & & & 17 & 0.173 \\
\hline Vac- & control & PBS & 14974 & 10 & 0 \\
\hline cination & & p.o. & & 12 & 0.587 \\
\hline study II & & & & 14 & 1.66 \\
\hline
\end{tabular}


Table A.3-3: Overview of the detected antibodies in A27 in vaccination study I. The samples of the animals were tested at different time points. When antibodies bound to a sequence of the A27 protein, the respective B cell epitope regions were marked red.
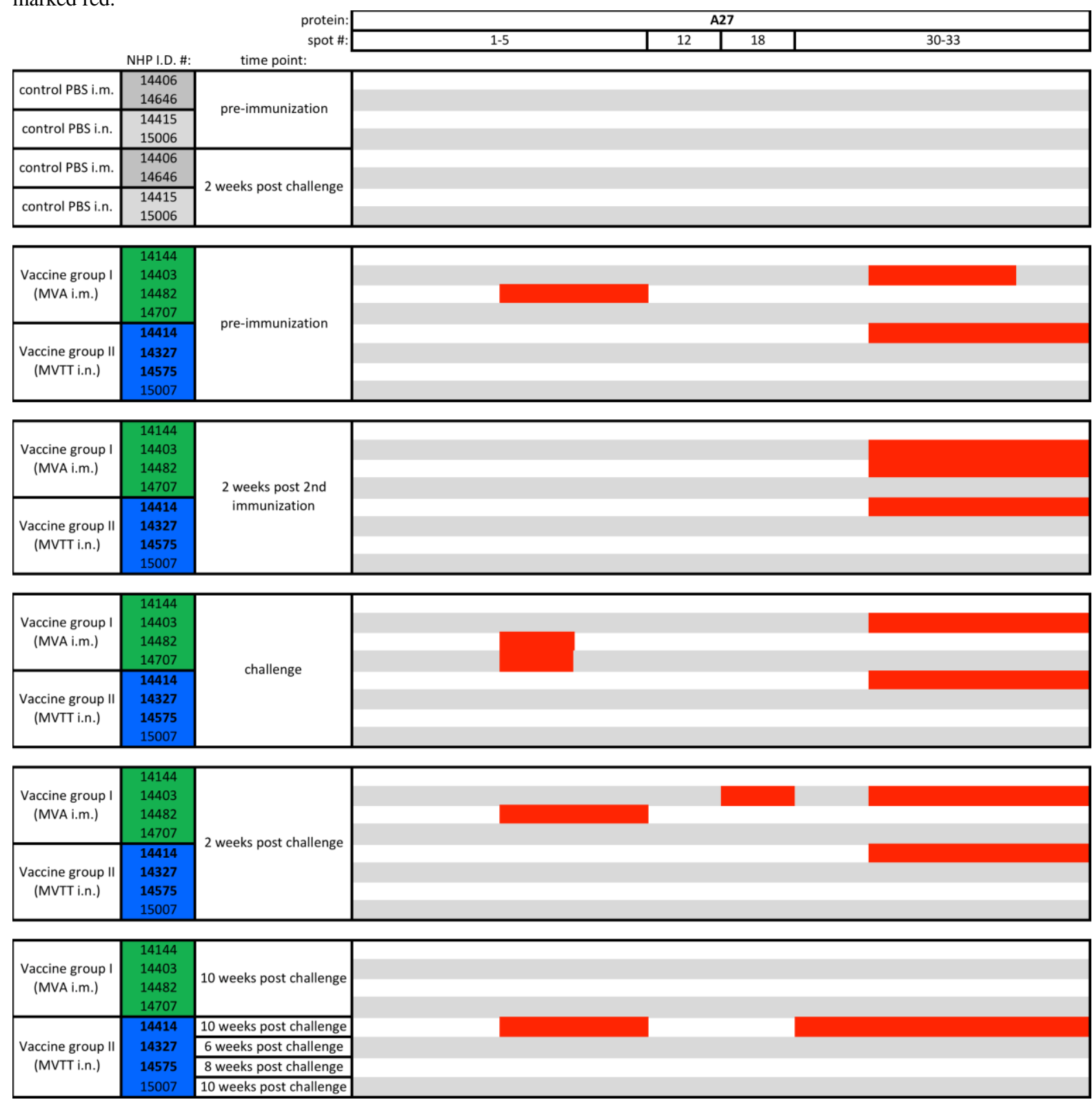

Bold numbers, protected animals; i.m., intramuscular Immunization; i.n., intranasal Immunization; MVA, modified vaccinia virus Ankara; MVTT, modified vaccinia virus Tiantan. 
Table A.3-4: Overview of the detected antibodies in D8 in vaccination study $\mathbf{I}$. The samples of the animals were tested at different time points. When antibodies bound to a sequence of the D8 protein, the respective B cell epitope regions were marked red.
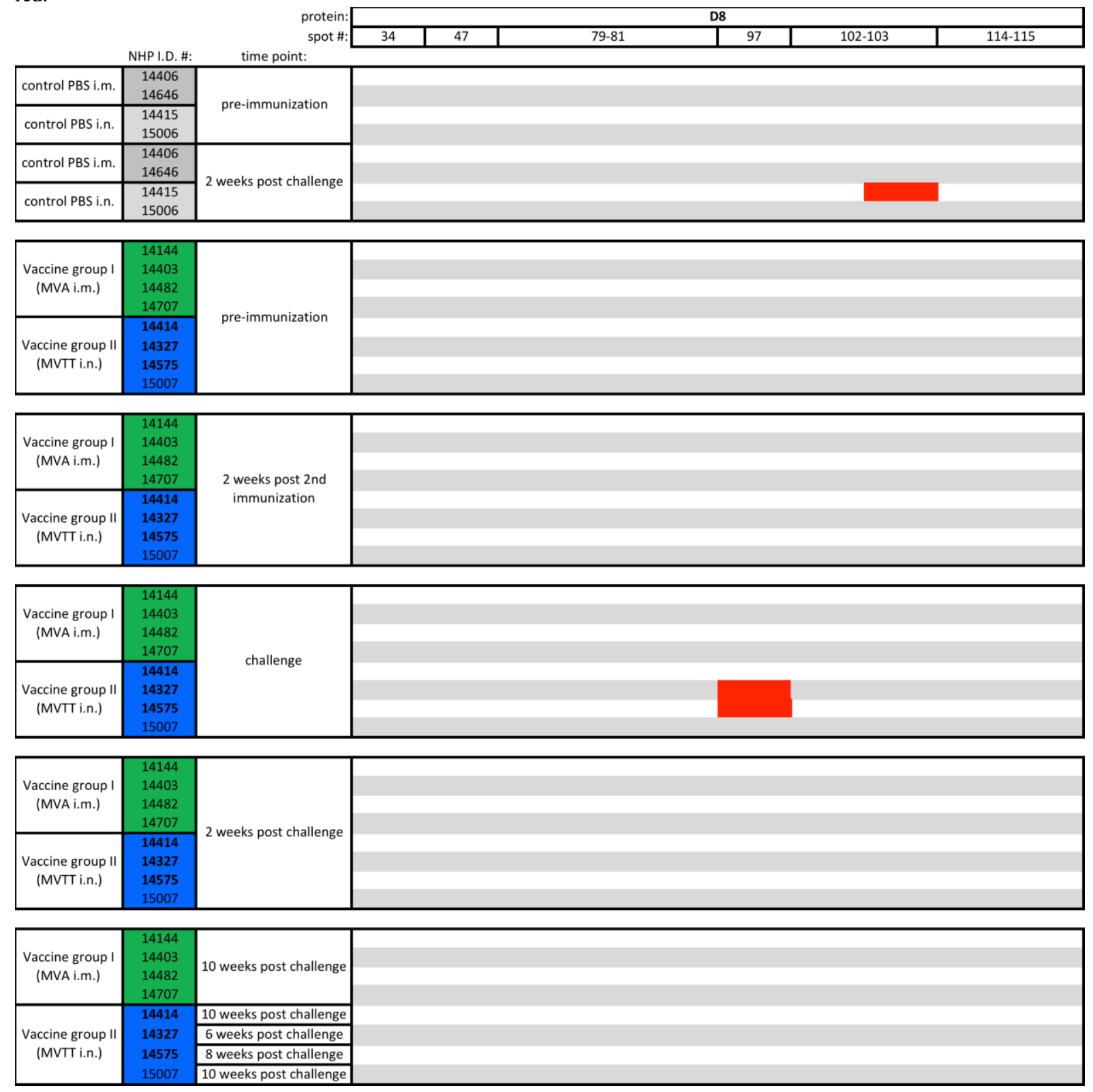

Bold numbers, protected animals; i.m., intramuscular Immunization; i.n., intranasal Immunization; MVA, modified vaccinia virus Ankara; MVTT, modified vaccinia virus Tiantan. 
Table A.3-5: Overview of the detected antibodies in $\mathbf{H 3}$ and $\mathrm{L1}$ in vaccination study $\mathrm{I}$. The samples of the animals were tested at different time points. When antibodies bound to a sequence of the H3 and L1 protein, the respective B cell epitope regions were marked red.

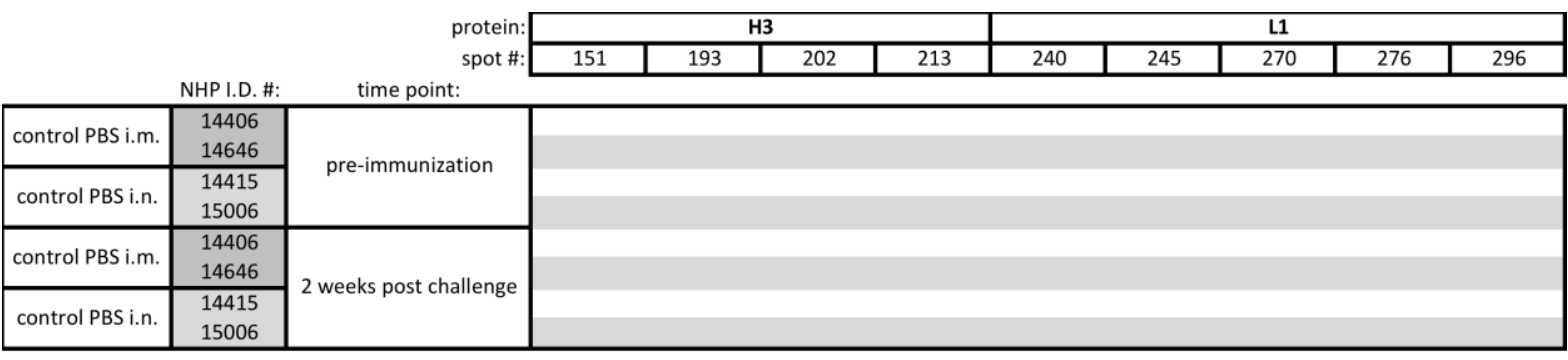

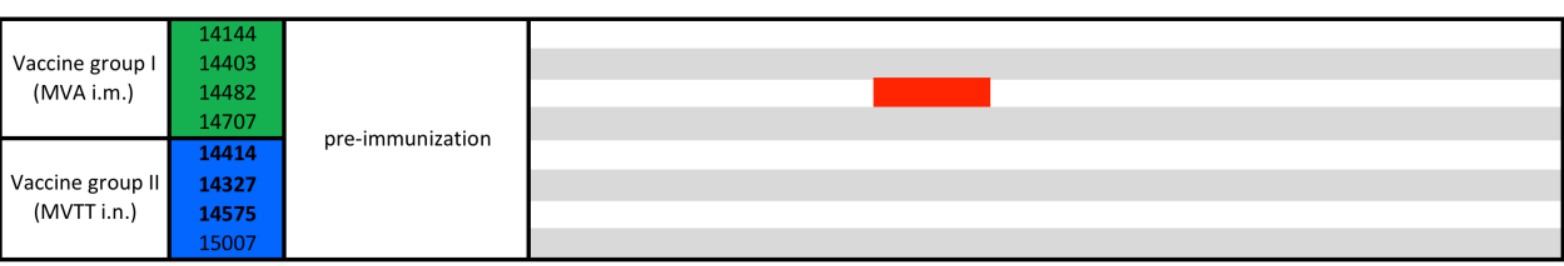
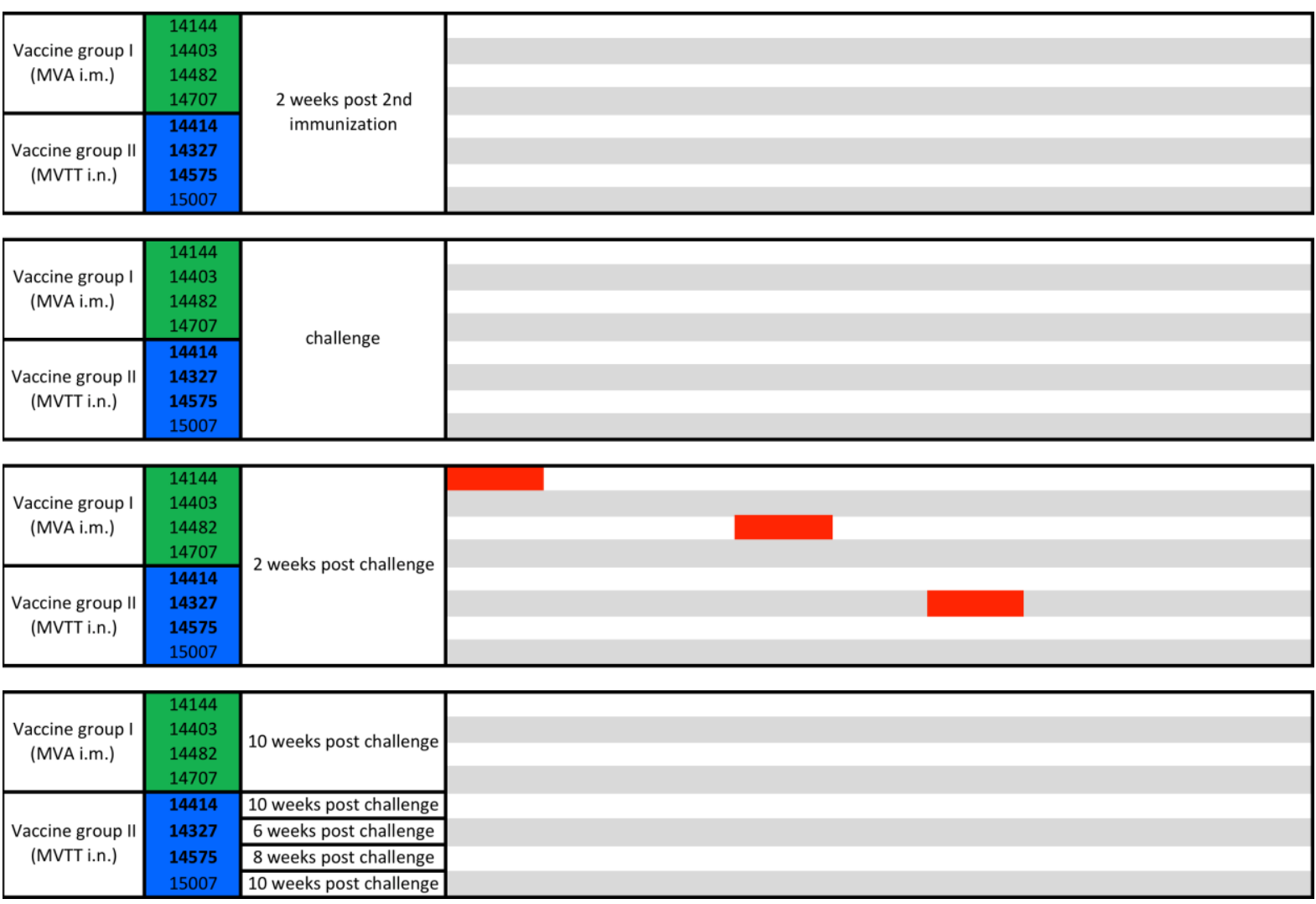

Bold numbers, protected animals; i.m., intramuscular Immunization; i.n., intranasal Immunization; MVA, modified vaccinia virus Ankara; MVTT, modified vaccinia virus Tiantan. 
Table A.3-6: Overview of the detected antibodies in A33 in vaccination study I. The samples of the animals were tested at different time points. When antibodies bound to a sequence of the A33 protein, the respective B cell epitope regions were marked red.

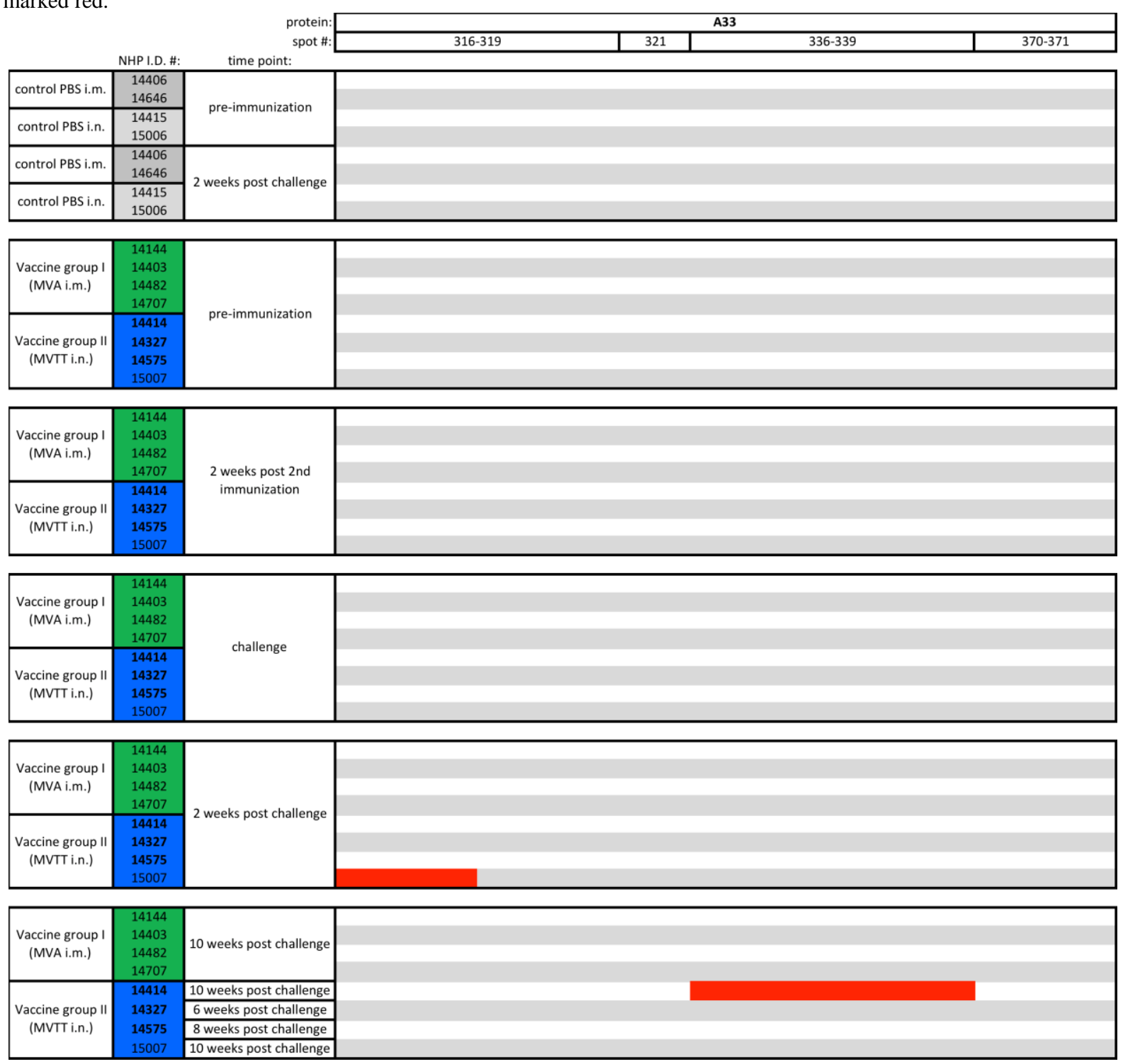

Bold numbers, protected animals; i.m., intramuscular Immunization; i.n., intranasal Immunization; MVA, modified vaccinia virus Ankara; MVTT, modified vaccinia virus Tiantan. 
Table A.3-7: Overview of the detected antibodies in B5 in vaccination study $\mathbf{I}$. The samples of the animals were tested at different time points. When antibodies bound to a sequence of the B5 protein, the respective B cell epitope regions were marked red.
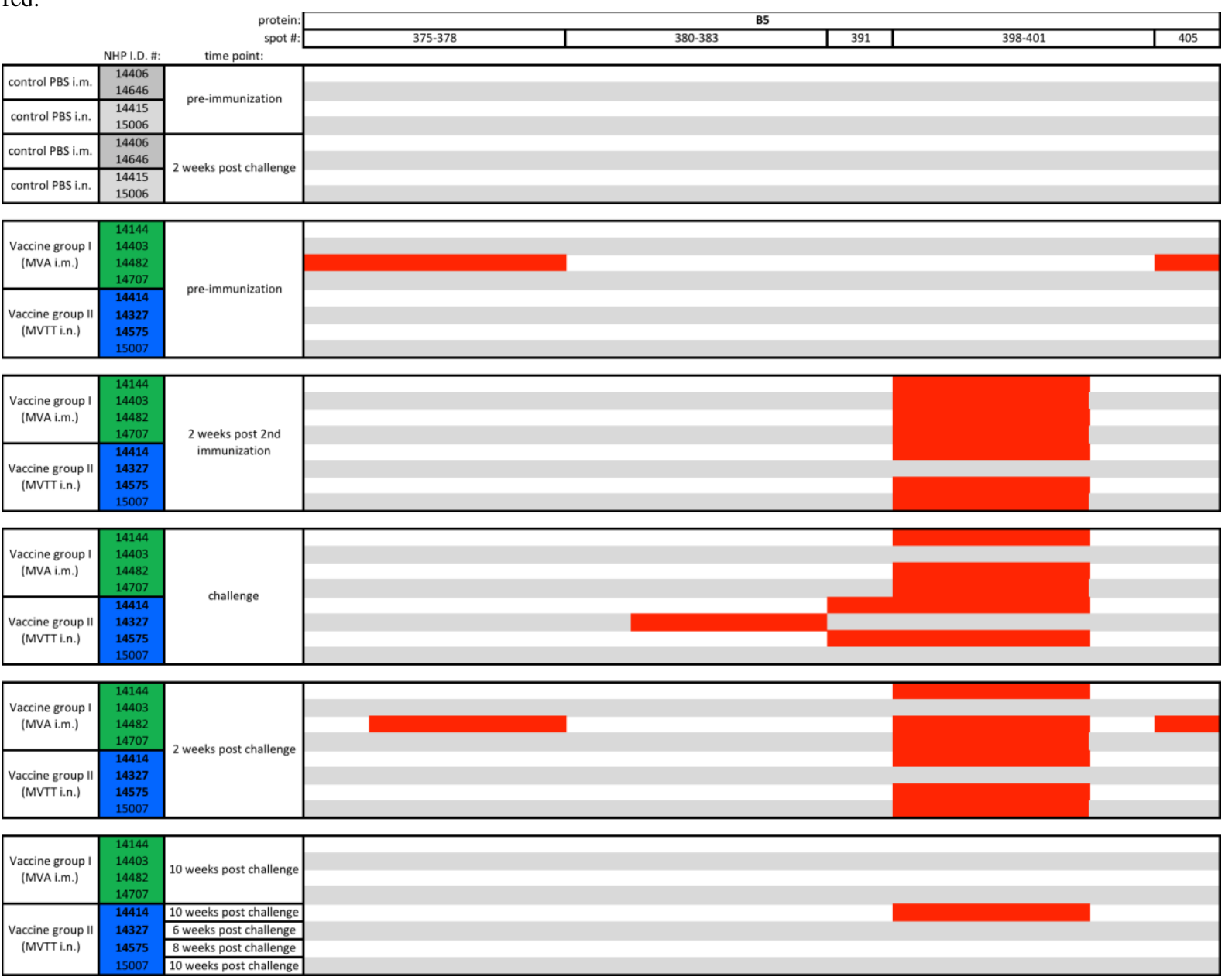

Bold numbers, protected animals; i.m., intramuscular Immunization; i.n., intranasal Immunization; MVA, modified vaccinia virus Ankara; MVTT, modified vaccinia virus Tiantan. 
Table A.3-8: Overview of the detected antibodies in $\mathbf{B 5}$ in vaccination study $\mathbf{I}$. The samples of the animals were tested at different time points. When antibodies bound to a sequence of the B5 protein, the respective B cell epitope regions were marked red.

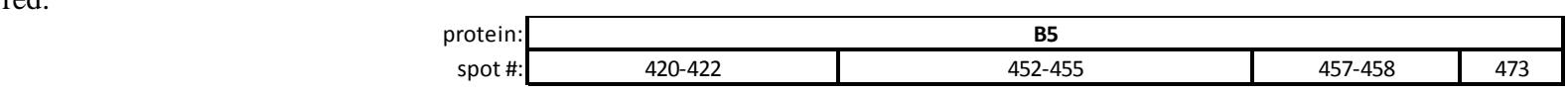

\begin{tabular}{|c|c|c|c|}
\hline & & \multirow{5}{*}{ pre-immunization } & \\
\hline & 14400 & & \\
\hline i.m. & 14646 & & \\
\hline control PBS & 14415 & & \\
\hline i.n. & 15006 & & \\
\hline control PBS & 14406 & \multirow{4}{*}{2 weeks post challenge } & \\
\hline i.m. & 14646 & & \\
\hline control PBS & 14415 & & \\
\hline i.n. & 15006 & & \\
\hline
\end{tabular}
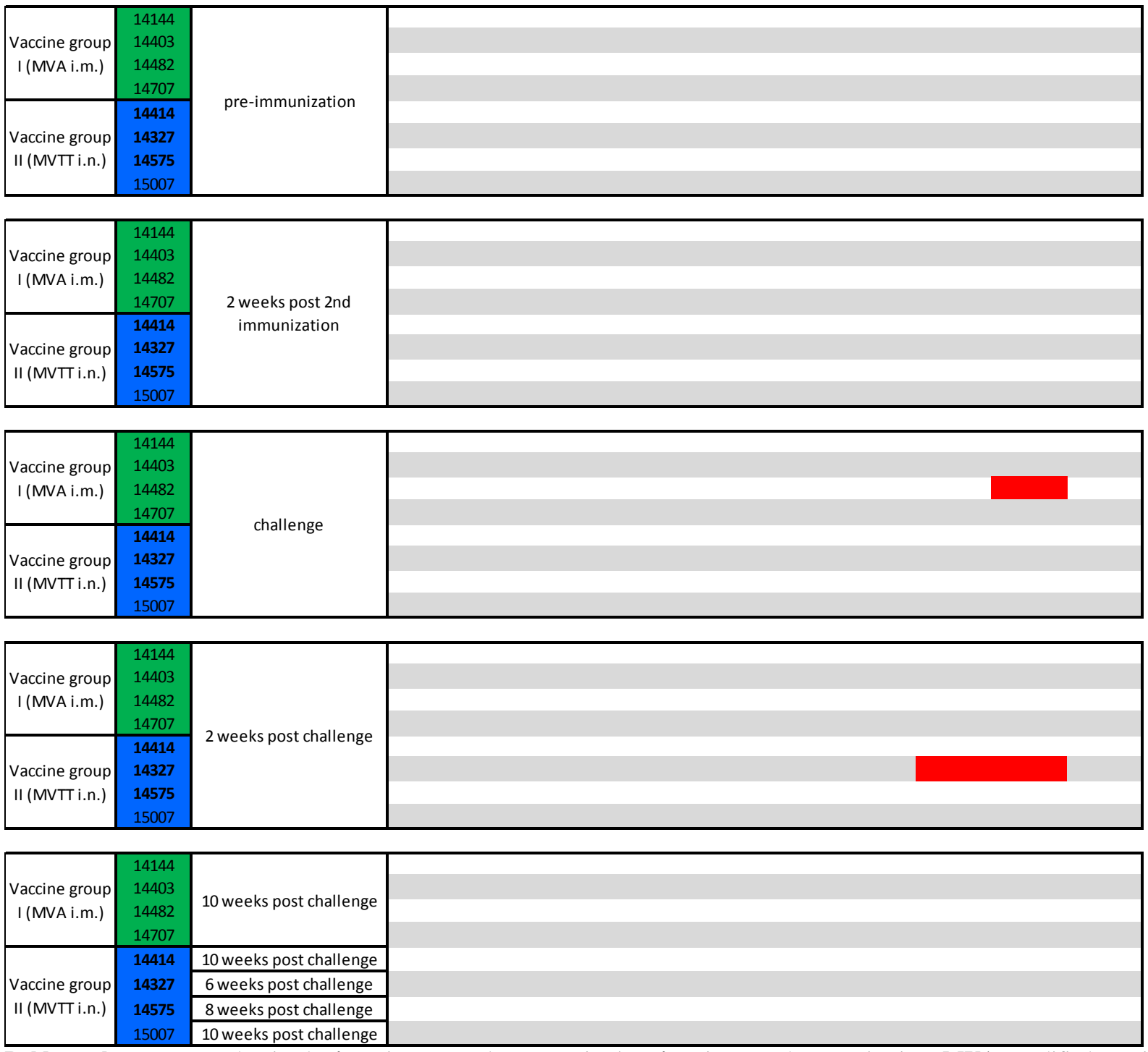

Bold numbers, protected animals; i.m., intramuscular Immunization; i.n., intranasal Immunization; MVA, modified vaccinia virus Ankara; MVTT, modified vaccinia virus Tiantan. 
Table A.3-9: Overview of the detected antibodies in A27 in vaccination study II. The samples of the animals were tested at different time points. When antibodies bound to a sequence of the A27 protein, the respective B cell epitope regions were marked red.

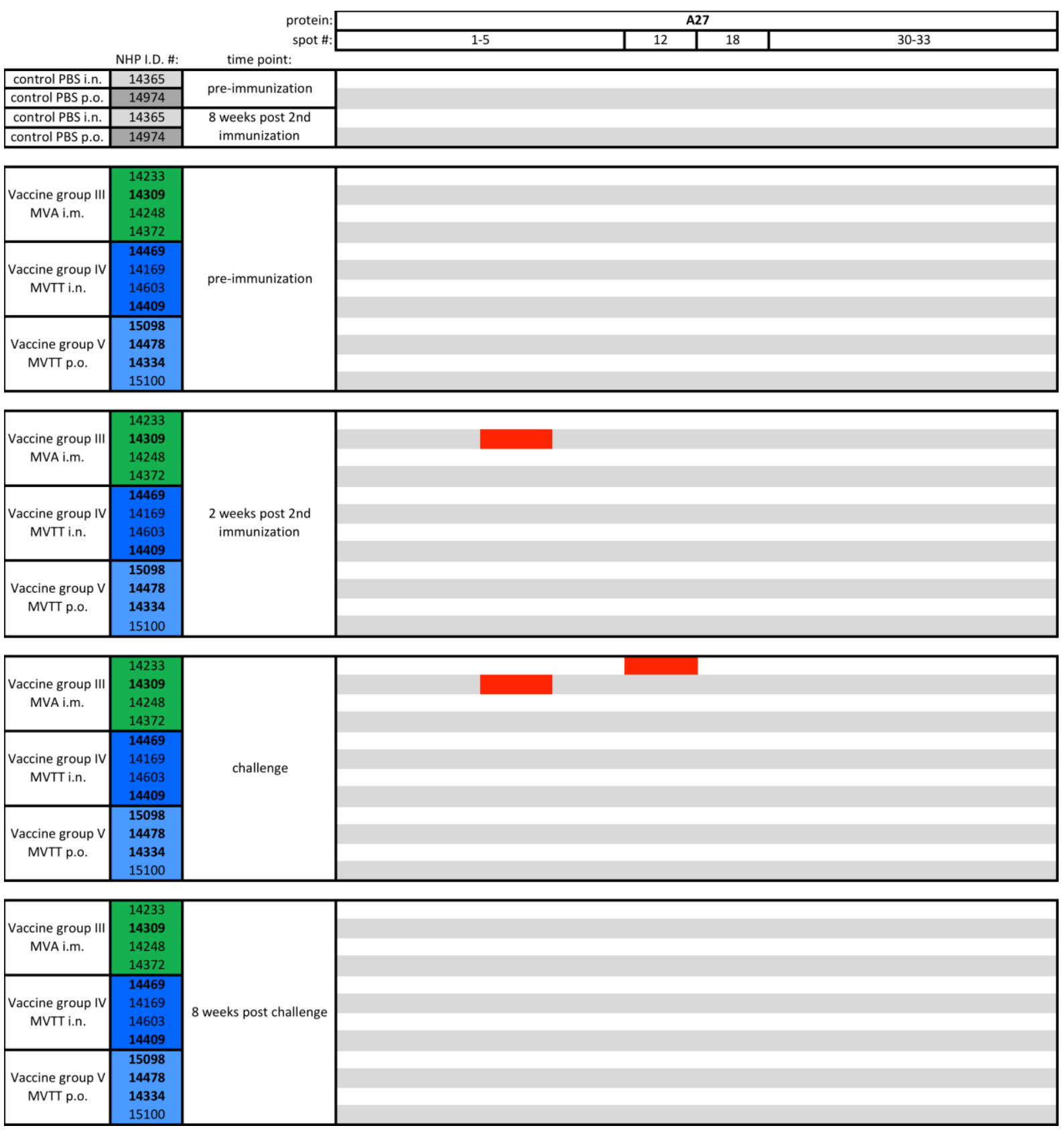

Bold numbers, protected animals; i.m., intramuscular Immunization; i.n., intranasal Immunization; MVA, modified vaccinia virus Ankara; MVTT, modified vaccinia virus Tiantan; p.o., per oral. 
Table A.3-10: Overview of the detected antibodies in D8 in vaccination study II. The samples of the animals were tested at different time points. When antibodies bound to a sequence of the D8 protein, the respective B cell epitope regions were marked red.

\begin{tabular}{|c|c|c|c|c|c|c|c|c|}
\hline & \multirow{2}{*}{\multicolumn{2}{|c|}{$\begin{array}{r}\text { protein: } \\
\text { spot \#: }\end{array}$}} & \multicolumn{6}{|c|}{ D8 } \\
\hline & & & 34 & 47 & $79-81$ & 97 & $102-103$ & $114-115$ \\
\hline & NHP I.D.\#: & time point: & & & & & & \\
\hline control PBS i.n. & 14365 & pre-immunization & & & & & & \\
\hline control PBS p.o. & 14974 & pre-Immunization & & & & & & \\
\hline control PBS i.n. & 14365 & 8 weeks post 2 nd & & & & & & \\
\hline control PBS p.o. & 14974 & immunization & & & & & & \\
\hline
\end{tabular}
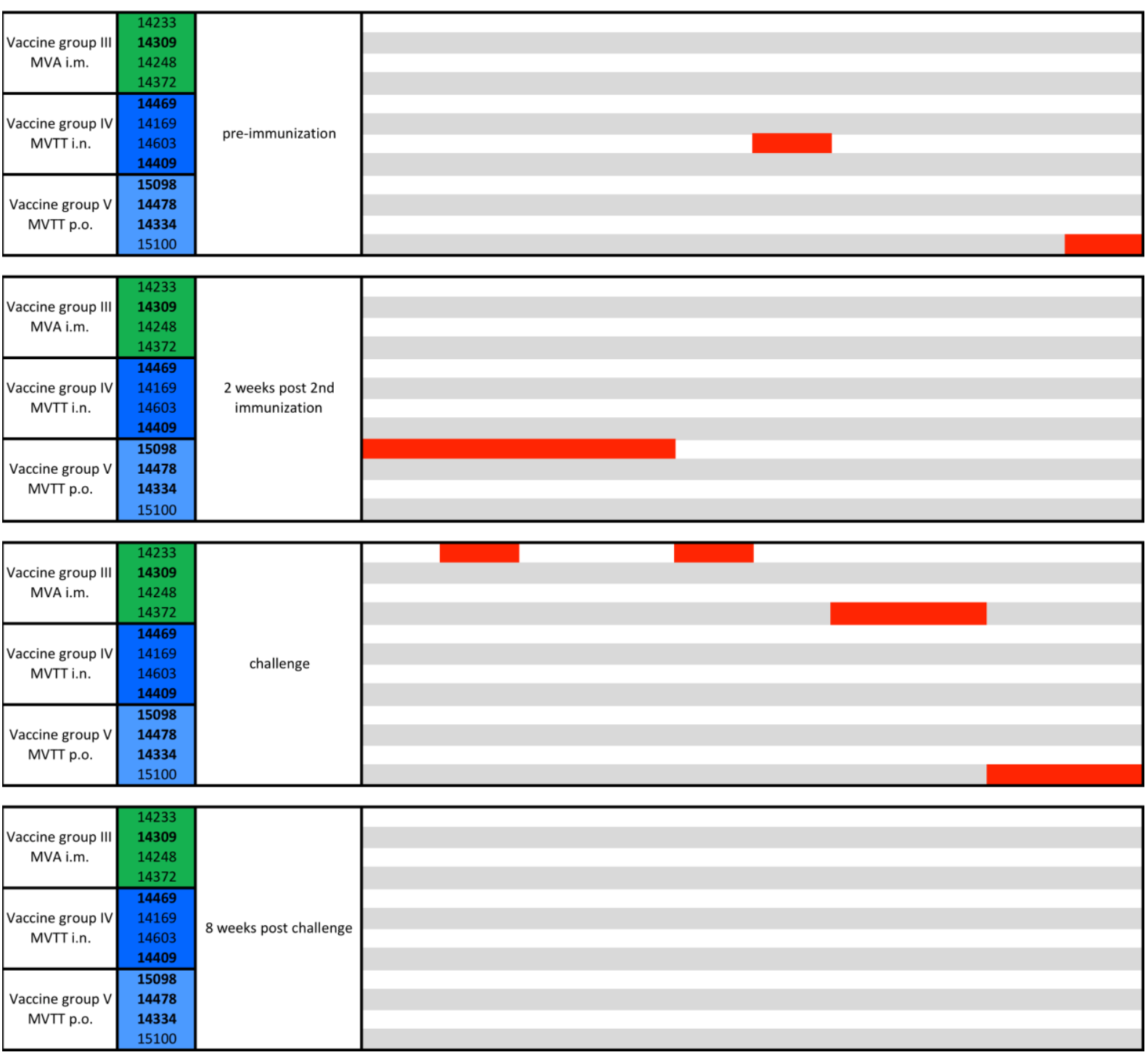

Bold numbers, protected animals; i.m., intramuscular Immunization; i.n., intranasal Immunization; MVA, modified vaccinia virus Ankara; MVTT, modified vaccinia virus Tiantan; p.o., per oral. 
Table A.3-11: Overview of the detected antibodies in $\mathbf{H 3}$ and $\mathrm{L1}$ in vaccination study II. The samples of the animals were tested at different time points. When antibodies bound to a sequence of the H3 and L1 protein, the respective B cell epitope regions were marked red.
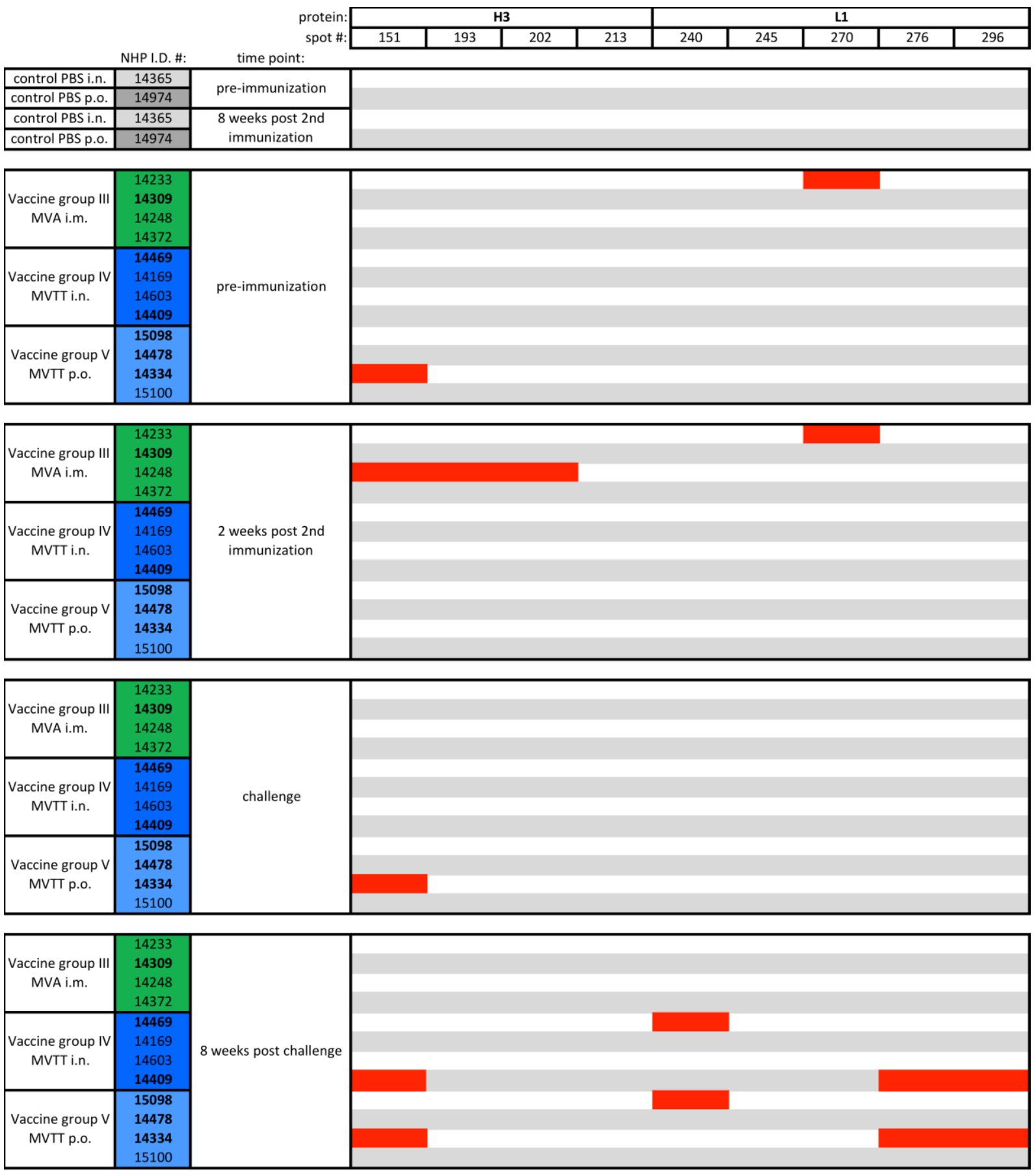

Bold numbers, protected animals; i.m., intramuscular Immunization; i.n., intranasal Immunization; MVA, modified vaccinia virus Ankara; MVTT, modified vaccinia virus Tiantan; p.o., per oral. 
Table A.3-12: Overview of the detected antibodies in A33 in vaccination study II. The samples of the animals were tested at different time points. When antibodies bound to a sequence of the A33 protein, the respective B cell epitope regions were marked red.
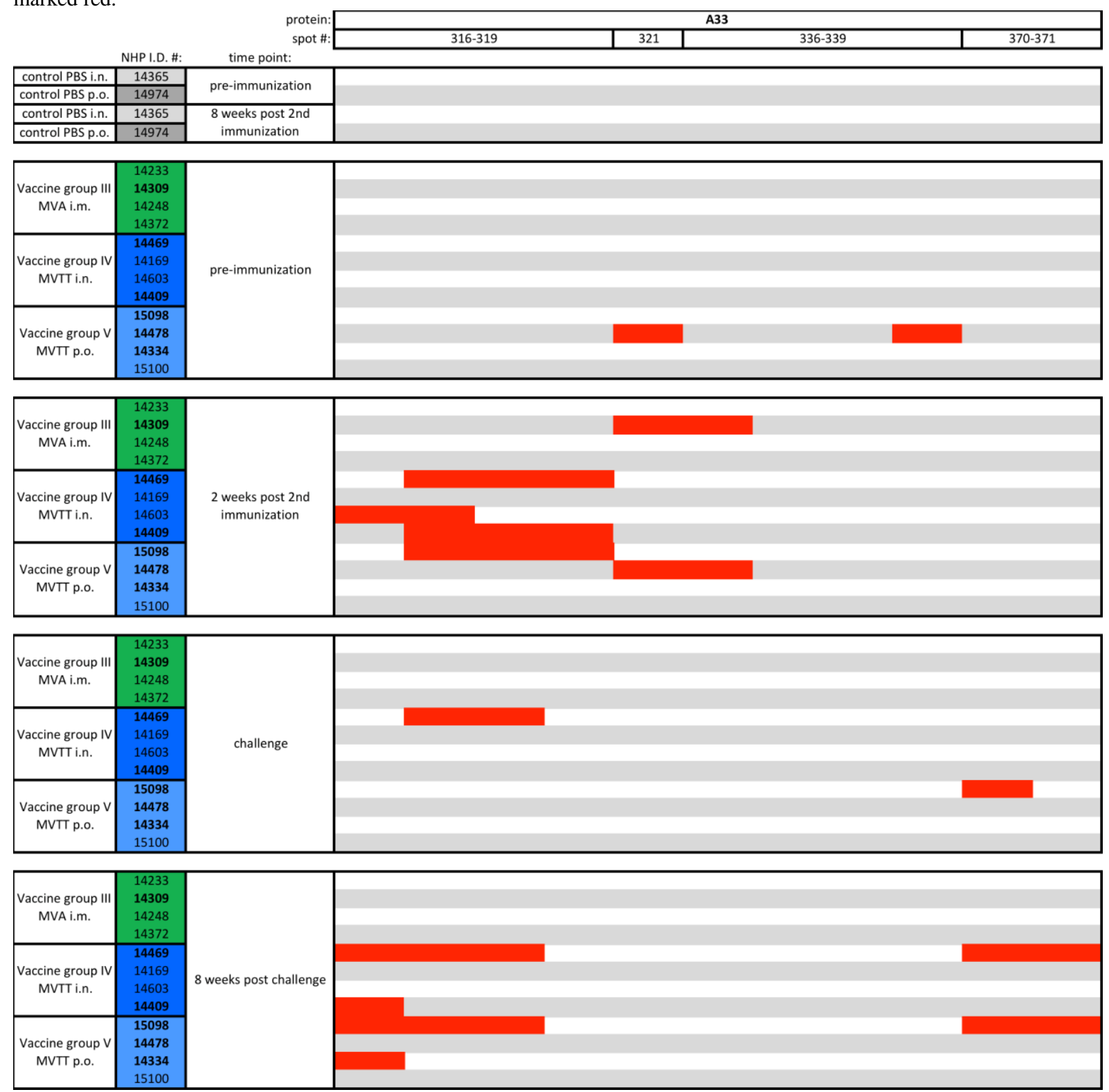

Bold numbers, protected animals; i.m., intramuscular Immunization; i.n., intranasal Immunization; MVA, modified vaccinia virus Ankara; MVTT, modified vaccinia virus Tiantan; p.o., per oral. 
Table A.3-13: Overview of the detected antibodies in B5 in vaccination study II. The samples of the animals were tested at different time points. When antibodies bound to a sequence of the B5 protein, the respective B cell epitope regions were marked red.
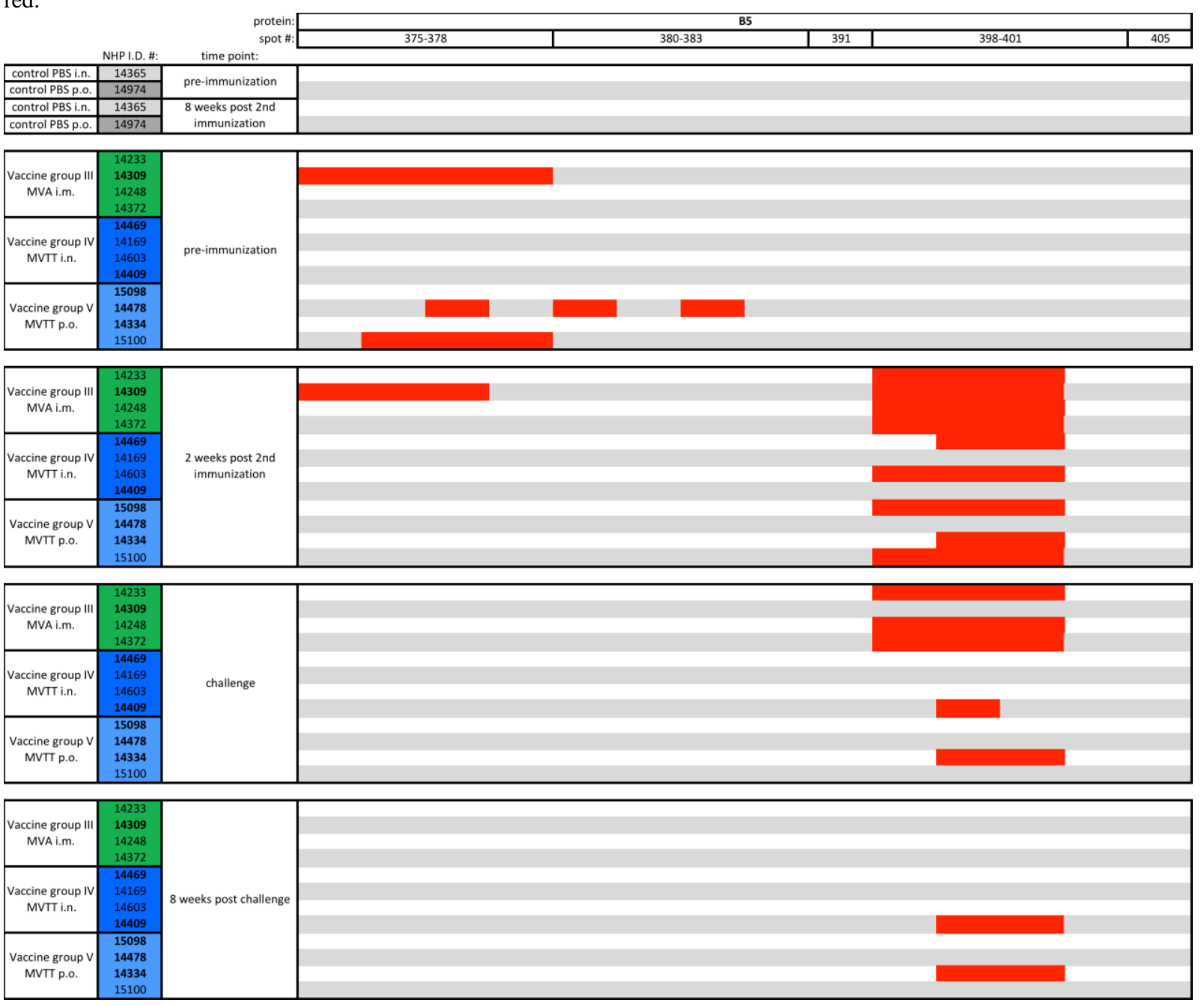

Bold numbers, protected animals; i.m., intramuscular Immunization; i.n., intranasal Immunization; MVA, modified vaccinia virus Ankara; MVTT, modified vaccinia virus Tiantan; p.o., per oral. 
Table A.3-14: Overview of the detected antibodies in B5 in vaccination study II. The samples of the animals were tested at different time points. When antibodies bound to a sequence of the B5 protein, the respective B cell epitope regions were marked red.

\begin{tabular}{|c|c|c|c|c|c|c|}
\hline & \multirow{2}{*}{\multicolumn{2}{|c|}{$\begin{array}{r}\text { protein: } \\
\text { spot \#: }\end{array}$}} & \multicolumn{4}{|c|}{ B5 } \\
\hline & & & $420-422$ & $452-455$ & $457-458$ & 473 \\
\hline & NHP I.D. \#: & \multicolumn{5}{|l|}{ time point: } \\
\hline control PBS i.n. & 14365 & \multirow{2}{*}{ pre-immunization } & & & & \\
\hline control PBS p.o. & 14974 & & & & & \\
\hline control PBS i.n. & 14365 & \multirow{2}{*}{$\begin{array}{c}8 \text { weeks post } 2 \text { nd } \\
\text { immunization }\end{array}$} & & & & \\
\hline control PBS p.o. & 14974 & & & & & \\
\hline \multirow{4}{*}{$\begin{array}{l}\text { Vaccine group } \\
\text { III MVA i.m. }\end{array}$} & 14233 & \multirow{12}{*}{ pre-immunization } & & & & \\
\hline & 14309 & & & & & \\
\hline & 14248 & & & & & \\
\hline & 14372 & & & & & \\
\hline \multirow{4}{*}{$\begin{array}{l}\text { Vaccine group } \\
\text { IV MVTT i.n. }\end{array}$} & 14469 & & & & & \\
\hline & 14169 & & & & & \\
\hline & 14603 & & & & & \\
\hline & 14409 & & & & & \\
\hline \multirow{4}{*}{$\begin{array}{c}\text { Vaccine group V } \\
\text { MVTT p.o. }\end{array}$} & 15098 & & & & & \\
\hline & 14478 & & & & & \\
\hline & 14334 & & & & & \\
\hline & 15100 & & & & & \\
\hline
\end{tabular}
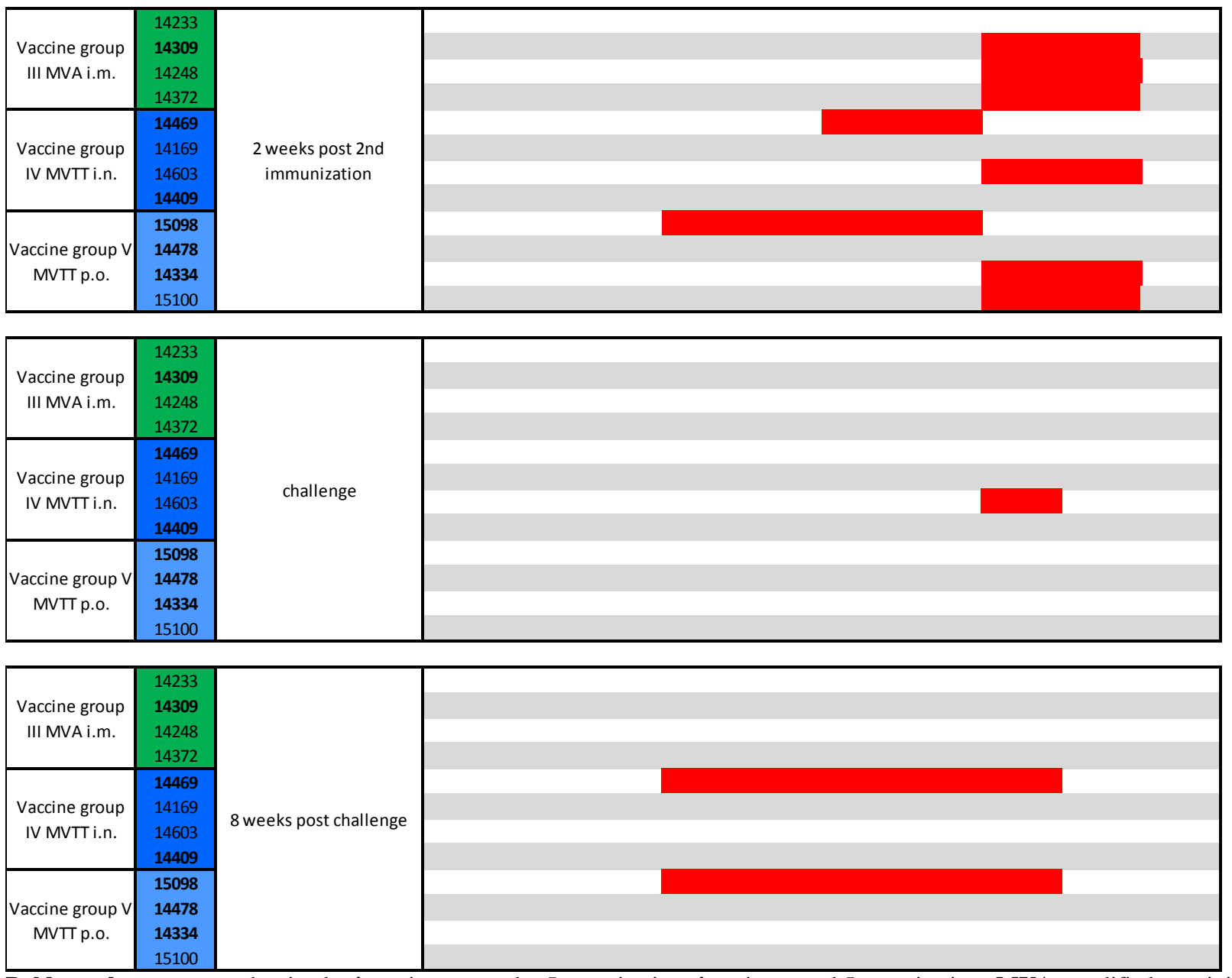

Bold numbers, protected animals; i.m., intramuscular Immunization; i.n., intranasal Immunization; MVA, modified vaccinia virus Ankara; MVTT, modified vaccinia virus Tiantan; p.o., per oral. 


\section{A.3 Danksagung}

Hiermit möchte ich mich bei all denjenigen bedanken, die mich im Rahmen der Dissertation und während der Zeit unterstützt haben.

Ein ganz besonderer Dank gilt meiner Doktormutter Dr. Christiane Stahl-Hennig für die uneingeschränkte Unterstützung, für unzählige Diskussionen und offene Sprechstunden. Vielen Dank für das Vertrauen und, dass dieses spannende Projekt in meine Hände gelegt wurde.

Desweiteren bedanke ich mich bei den Betreuern und Gutachtern dieser Arbeit, Dr. Christiane Stahl-Hennig, Prof. Dr. Dr. Claus-Peter Czerny und Prof. Dr. Stefan Becker. Danke für die hervorragende, fachliche Betreuung.

Ich danke der „Leibniz Graduate School for Emerging Infectious Diseases (EIDIS)“ für die finanzielle Unterstützung des Projektes. Außerdem danke ich der „Göttingen Graduate School for Neurosciences, Biophysics, and Molecular Biosciences (GGNB)“ für die finanzielle Unterstützung in Form des Überbrückungsstipendiums.

Vielen Dank an Tingchuan Shi für die fließende und reibungslose Übergabe dieses Projektes an mich und Shereen Petersen für die freundliche und herzliche Aufnahme in die Arbeitsgruppe.

Ein besonderer Dank gebührt Dr. Berit Neumann. Die sowohl technische als auch fachliche Hilfestellung war für das Erstellen dieser Dissertation, vor allem bei dem Erstellen der durchflusszytometrischen Daten, unentbehrlich.

Bei der Einführung in die überaus interessante Methode des Peptide Microarrays bedanke ich mich recht herzlich bei Dr. Sven-Kevin Hotop und Dr. Ahmed Abd El Wahed. Vielen Dank an Dr. Gabriela Salinas-Riester und die Möglichkeit der Nutzung des Agilent DNA microarray scanner (Agilent Technologies, USA) am Transkriptomanalyselabor der „DNA Microarray Facility, University Medical Center Göttingen“. Danke an Susanne Luthin und Fabian Ludewig für die freundliche Hilfestellung vor Ort.

Desweiteren bedanke ich mich bei Dr. Kerstin Mätz-Rensing von der Abteilung Infektionspathologie für die wertvollen Ratschläge und überaus freundliche Kooperation. Ein großer Dank auch an Dr. Anne Schmitt für die unkomplizierte, effektive und freundschaftliche Zusammenarbeit im Rahmen der parallel verlaufenden Doktorarbeit.

Außerdem bedanke ich mich herzlichst bei all denjenigen, die an der Planung und Durchführung der Tierexperimente beteiligt waren. Ein Dank gebührt den Tierärzten der Abteilung In- 
fektionsmodelle, Maria Daskalaki und Dr. Antonina Klippert sowie allen Tierpflegern, deren Hilfe bei der Durchführung der Tierexperimente essentiellen Wert hatte.

Ich möchte mich außerdem recht herzlichst bei Sandra Heine, Judith Hampe und Kerstin Eckelmann für die Einführung in das DPZ-Labor bedanken und, dass mir bei Fragen jederzeit hilfsbereit und offen zur Seite gestanden wurde. Danke für die überaus angenehme Arbeitsatmosphäre im Labor 305.

Für die Kooperation mit der Abteilung Mikrobiologie und Tierhygiene an der Georg-AugustUniversität Göttingen danke ich Prof. Dr. Dr. Claus-Peter Czerny, sowie Caroline Bierschenk und Sebastian Schimkowiak. Vielen Dank für die Einführung in das Mikrobiologie und Tierhygiene-Labor sowie die kompetente Anleitung hinsichtlich der Vorbereitung des ELISA und die gehaltvollen Diskussionen.

Ich danke Dr. Christiane Stahl-Hennig herzlichst für die Möglichkeit der Fortbildung. Die Durchführung von Lehre sowie die Teilnahmen an diversen GGNB-Kursen, Seminaren und Kongressen waren für mich sehr lehrreich.

Außerdem danke ich der gesamten Abteilung Infektionsmodelle am Deutschen Primatenzentrum, Shereen, Nicole L., Bianka, Christina, Heidi, Nicole S., Maria, Berit, Matthias, Sven, Kerstin, Antonina, Aila, Ulrike, Sebastian, Ahmad, Sandra, Sabine, Judith, Dirk, Barbara, Georgia, Nina und Ahmed für die Begleitung während der Zeit der Doktorarbeit, sowie Diskussionen und für die angenehme Arbeitsatmosphäre.

Ein großer Dank gebührt außerdem Shereen Petersen für die sprachliche Unterstützung.

Vielen Dank an die soziale, arbeitsgruppenübergreifende Doktorandenrunde und dem damit verbundenen „EIDIS-Graduate School“-Stammtisch, sowie unzählige, gemeinsame Ausflüge und Unternehmungen ins Kino, Theater, Restaurant und das sonstige göttingener Nachtleben.

Außerdem danke ich der kreativen „Mädchen“-runde. Sunny, Julia, Luzie, Maria und Johanna, danke für die überaus angenehme Gesellschaft, anregenden Gespräche, Feiereien, Inspiration für neue Projekte und vor allem eure Freundschaft.

Herzlichst möchte ich mich außerdem bei meinen Mitbewohnerinnen bedanken, die mich durch die Zeit der Doktorarbeit begleitet haben und mit denen ich eine sehr schöne und bereichernde Zeit verbracht habe.

Ein ganz besonderer Dank gebührt meiner Familie, meinen Eltern, sowie allen meinen Freunden in Norddeutschland, in Gießen und den neu dazu gewonnenen Freunden in Göttingen. 
Danke für gemeinsames Reisen und das Teilen meiner Leidenschaften für all die schönen, inspirierenden Dinge in Leben. Für eure Unterstützung in wohl jeder Lebenslage bin ich unglaublich dankbar. Ihr nehmt einen nicht unwesentlichen Teil in meinem Leben ein und habt somit zum Gelingen dieser Arbeit beigetragen. Danke* 


\section{A.4 List of Publications and Presentations}

\section{Journals}

Anne Schmitt*, Li Lin Gan*, Christiane Stahl-Hennig, Tingchuan Shi, Heinz Ellerbrok, Franz-Josef Kaup, Kerstin Mätz-Rensing; *Authors with equal contribution, Dynamics of pathological findings during experimental calpox virus infection of common CM (Callithrix jacchus); 2017; Viruses; accepted; article

Berit Neumann, Tingchuan Shi, Li Lin Gan, Antonina Klippert, Maria Daskalaki, Nicole Stolte-Leeb, Christiane Stahl-Hennig; Comprehensive panel of cross-reacting monoclonal antibodies for analysis of different immune cells and their distribution in the common marmoset (Callithrix jacchus); 2016; Journal of Medical Primatology doi: 10.1111/jmp.12216; article

Li Lin Gan, Stefan T. Hertwig, Indraneil Das, Alexander Haas; The anatomy and structural connectivity of the abdominal sucker in the tadpoles of Huia cavitympanum, with comparisons to Meristogenys jerboa (Lissamphibia: Anura: Ranidae); 2015; J Zoolog Syst Evol Res doi: 10.1111/jzs.12113; article

\section{Conference Contribution}

${\underline{\mathrm{Li}} \mathrm{Lin} \mathrm{Gan}^{1}}^{1}$, Tingchuan $\mathrm{Shi}^{2}$, Dirk Motzkus ${ }^{2}$, Berit Neumann ${ }^{2}$, Claus-Peter Czerny ${ }^{3}$, FranzJosef Kaup $^{4}$, Kerstin Mätz-Rensing ${ }^{4}$, Zhiwei Chen ${ }^{5}$, Heinz Ellerbrok ${ }^{6}$, Christiane StahlHennig $^{2}$

1.Unit of Infection Models, German Primate Center, Goettingen, Germany, 2.Unit of Infection Models, German Primate Center, Goettingen, Germany, 3.Department of Animal Sciences, University of Göttingen, Goettingen, Germany, 4.Pathology Unit, German Primate Center, Goettingen, Germany, 5.Aids Institute and Department of Microbiology, University of Hong Kong, Hong Kong, China , 6.Robert Koch Institute, Robert Koch Institute, Berlin, Germany, Studies on orthopoxvirus pathogenesis and vaccine efficacy assessment using the calpoxmarmoset model; Joint Conference: National German Symposium on Zoonoses Research 2014 and $7^{\text {th }}$ International Conference on Emerging Zoonoses, 16-17 October 2014 in Berlin, Germany; poster presentation

$\underline{\mathrm{Li}} \mathrm{Lin} \mathrm{Gan}^{1}$, Anne Schmitt ${ }^{2}$, 1.Unit of Infection Models, German Primate Center, Goettingen, Germany, 2.Pathology Unit, German Primate Center, Goettingen, Germany, CalpoxInfection in CM; Goettingen Virology Network, 05 November 2014 in Goettingen, Germany; talk

$\underline{\mathrm{Li}}$ Lin Gan $^{1}$, 1.Unit of Infection Models, German Primate Center, Goettingen, Germany, The use of poxvirus microarray scanning chip in the orthopoxvirus marmoset model; Peptide Microarrays and High Resolution Serological Analysis, 5-7 June 2015 in Goettingen, Germany; talk

$\underline{\text { Li Lin Gan }}{ }^{1}$, Tingchuan $\mathrm{Shi}^{2}$, Dirk Motzkus ${ }^{2}$, Berit Neumann ${ }^{2}$, Claus-Peter Czerny ${ }^{3}$, FranzJosef Kaup ${ }^{4}$, Kerstin Mätz-Rensing ${ }^{4}$, Zhiwei Chen ${ }^{5}$, Heinz Ellerbrok ${ }^{6}$, Christiane StahlHennig $^{2}$

1.Unit of Infection Models, German Primate Center, Goettingen, Germany, 2.Unit of Infection Models, German Primate Center, Goettingen, Germany, 3.Department of Animal Sciences, University of Göttingen, Goettingen, Germany, 4.Pathology Unit, German Primate Center, 
Goettingen, Germany, 5.Aids Institute and Department of Microbiology, University of Hong Kong, Hong Kong, China , 6.Robert Koch Institute, Robert Koch Institute, Berlin, Germany, Pathogenesis of orthopoxvirus infection and vaccine efficacy assessment using the calpox-marmoset model; Junior Scientist Zoonoses Meeting, 8-10 June 2015 in Munich, Germany; poster presentation

$\underline{\text { Li Lin Gan }}{ }^{1}$, Tingchuan $\mathrm{Shi}^{2}$, Dirk Motzkus ${ }^{2}$, Berit Neumann ${ }^{2}$, Claus-Peter Czerny ${ }^{3}$, FranzJosef Kaup ${ }^{4}$, Kerstin Mätz-Rensing ${ }^{4}$, Zhiwei Chen ${ }^{5}$, Heinz Ellerbrok ${ }^{6}$, Christiane StahlHennig $^{2}$

1.Unit of Infection Models, German Primate Center, Goettingen, Germany, 2.Unit of Infection Models, German Primate Center, Goettingen, Germany, 3.Department of Animal Sciences, University of Göttingen, Goettingen, Germany, 4.Pathology Unit, German Primate Center, Goettingen, Germany, 5.Aids Institute and Department of Microbiology, University of Hong Kong, Hong Kong, China , 6.Robert Koch Institute, Robert Koch Institute, Berlin, Germany, Studies on pathogenesis of orthopoxvirus (OPXV) infection and vaccine efficacy of different attenuated smallpox vaccines in the calpox-marmoset model; Junior Scientist Zoonoses Meeting, 01-03 June 2016 in Goettingen, Germany; poster presentation

$\underline{\text { Li Lin Gan }}{ }^{1}$, Tingchuan $\mathrm{Shi}^{2}$, Dirk Motzkus ${ }^{2}$, Berit Neumann ${ }^{2}$, Claus-Peter Czerny ${ }^{3}$, FranzJosef Kaup ${ }^{4}$, Kerstin Mätz-Rensing ${ }^{4}$, Zhiwei Chen ${ }^{5}$, Heinz Ellerbrok ${ }^{6}$, Christiane StahlHennig $^{2}$

1.Unit of Infection Models, German Primate Center, Goettingen, Germany, 2.Unit of Infection Models, German Primate Center, Goettingen, Germany, 3.Department of Animal Sciences, University of Göttingen, Goettingen, Germany, 4.Pathology Unit, German Primate Center, Goettingen, Germany, 5.Aids Institute and Department of Microbiology, University of Hong Kong, Hong Kong, China , 6.Robert Koch Institute, Robert Koch Institute, Berlin, Germany, Studies on pathogenesis of orthopoxvirus (OPXV) infection and vaccine efficacy of different attenuated smallpox vaccines in the calpox-marmoset model; XXI International Poxvirus, Asfavirus and Iridovirus Conference, 01-06 July 2016 in Strasbourg, France; talk 


\section{A.5 Curriculum Vitae}

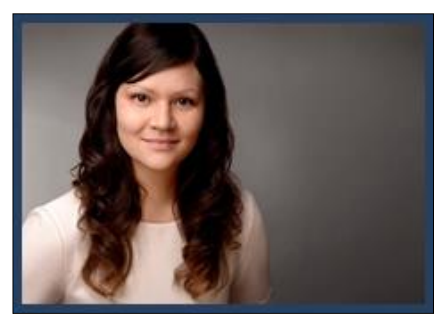

\section{Personal Details}

Name: $\quad$ Li Lin Gan

Date of birth: $\quad 10^{\text {th }}$ May 1988

Place of birth: Geesthacht, Germany

Nationality: German

\section{Education}

$2014-2018$

Georg-August-University Göttingen, Göttingen

Leibniz Graduate School for Emerging Infectious Diseases (EIDIS)

Göttingen Graduate School for Neurosciences, Biophysics and Molecular Biosciences (GGNB)

Dr. rer. nat., Biology

Thesis: $\quad$ Pathogenesis of orthopoxvirus (OPV) infection in common $C M$ and

identification of immune correlates after vaccination with differently attenuated vaccines

Supervisor: Dr. Christiane Stahl-Hennig

$2011-2013 \quad$ Justus-Liebig-University Gießen, Gießen

M.Sc., Biology

Thesis: $\quad$ The larval cranium of Huia cavitympanum (Boulenger, 1893) (Amphibia: Ranidae) and its bearing on the phylogeny of Huia

Supervisor: Prof. Dr. Alexander Haas

Final grade: $\quad 13.4=1.2($ very good $)$

$2009-2011 \quad$ Justus-Liebig-University Gießen, Gießen

B.Sc., Biology

Thesis: $\quad$ From barcodes to species lists: DNA-based species identification of anurans at a Cerrado site in Bolivia

Supervisor: Dr. habil. Gunther Köhler

\section{Professional Qualification}

2007 - $2009 \quad$ Staatliche Gewerbeschule 13, Nettelnburg

Biological-Technical Assistent in Biochemistry

Main area: Biochemistry

\section{Professional Career}


Entomology

Academic assistant; Advisor: Dr. Frank Friedrich

2013 - $2013 \quad$ University Hamburg, Hamburg

Ornithological collection

Academic assistant; Advisor: Prof. Dr. Alexander Haas

$2012-2012$

Justus-Liebig-University Gießen, Gießen

Administration

Academic assistant; Advisor: Ms. Regina Gaitsch 


\section{Declaration of honour}

I hereby declare that this submission is my own work and that, to the best of my knowledge and belief, it contains no material previously published or written by another person nor material which to a substantial extent has been accepted for the award of any other degree of the university or other institute of higher education, except where due acknowledgment has been made in the text. I have used no other than the stated sources and aids. All used textual or content-related citations and used diagrams are detected with source citations.

Göttingen, 05. Dezember 2017

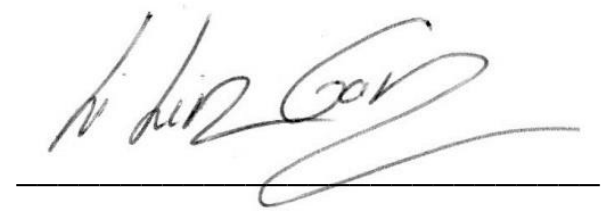

Li Lin Gan 